University of New Hampshire

University of New Hampshire Scholars' Repository

Data Catalog

2021

\title{
New Hampshire Continental Shelf Geophysical Database: \\ 2016-2017 Field Campaign - Seafloor and Sample Photographs and Sediment Data
}

\author{
Larry G. Ward \\ University of New Hampshire, Durham, Igward@ad.unh.edu \\ Rachel C. Morrison \\ University of New Hampshire, Durham, rmorrison@ccom.unh.edu \\ Zachary S. McAvoy \\ University of New Hampshire, Durham
}

Follow this and additional works at: https://scholars.unh.edu/data_series

Part of the Geology Commons, Oceanography Commons, and the Sedimentology Commons

\begin{abstract}
Recommended Citation
Ward, L.G., Morrison, R.C., and McAvoy, Z.S., 2021, New Hampshire Continental Shelf Geophysical Database: 2016-2017 Field Campaign - Seafloor and Sample Photographs and Sediment Data. University of New Hampshire Center for Coastal and Ocean Mapping/Joint Hydrographic Center (CCOM/JHC), 24 Colovos Road, Durham 03824.
\end{abstract}

This Data Set is brought to you for free and open access by University of New Hampshire Scholars' Repository. It has been accepted for inclusion in Data Catalog by an authorized administrator of University of New Hampshire Scholars' Repository. For more information, please contact Scholarly.Communication@unh.edu. 


\section{New Hampshire Continental Shelf Geophysical Database: 2016-2017 Field Campaign - Seafloor and Sample Photographs and Sediment Data}

\section{Citation:}

The data presented here is freely available. However, please cite the following reference when using: Ward, L.G., Morrison, R.C., and McAvoy, Z.S., 2021, New Hampshire Continental Shelf Geophysical Database: 2016-2017 Field Campaign - Seafloor and Sample Photographs and Sediment Data. University of New Hampshire Center for Coastal and Ocean Mapping/Joint Hydrographic Center (CCOM/JHC), 24 Colovos Road, Durham 03824. UNH Scholars Repository (https://scholars.unh.edu/).

\section{Database Description:}

The "New Hampshire Continental Shelf Geophysical Database: 2016-2017 Field Campaign - Seafloor and Sample Photographs and Sediment Data " contains photographs of the seafloor from sampling locations, photographs of the sediment samples, and grain size data from a major field campaign conducted in 20162017 and from the UNH Ocean Engineering 972 Hydrographic Field Course classes in 2012, 2014, and 2018. In total, sixteen one-day cruises provided 150 samples for grain size analysis. The database provides complete descriptions for each sample including identification, station and sample characteristics, sediment classifications, grain size statistics, and grain size distribution. The grain size data is grouped by survey or location and the year when high-resolution multibeam echosounder bathymetry was run in that area. The Station IDs indicate the type of survey (e.g., SH stands for Summer Hydro) and/or location (NSBE), the year of the bathymetry survey (i.e., 06, 07, etc.), and the station number (e.g., T03, T04, etc. or S01, S02, etc.). Each sample has a "Reliability Ranking for Positioning" which gives an estimate of the uncertainty of the location of the sample classified from 1 to 4 . Explanation of uncertainty and the numbering system is given in Ward et al. (2021). Briefly, the uncertainty is as follows: category 1 is $<30 \mathrm{~m}$; category 2 is 30 to $50 \mathrm{~m}$; category 3 is 185 to $463 \mathrm{~m}$; and category 4 is 250 to 1000

The stations sampled during the New Hampshire Continental Shelf 2016-2017 Field Campaign were targeted at locations where there was high-resolution bathymetry available. The bathymetry includes MBES surveys conducted by the UNH CCOM/JHC Hydrographic Field Course (Ocean Engineering 972) (all GSH or SH), NOS surveys (NSBE), and lidar surveys by the USGS (Shoals) (see Ward et al., 2021). In this database the stations are grouped by survey or location and year the bathymetry survey was run. The Station IDs indicate the type of survey (e.g., SH, GSH, Shoals) and/or location (NSBE), the year of the bathymetry survey (i.e., 06, 07, etc.), and the station number (e.g., T03, T04, etc. or S01, S02, etc.). In total, sixteen one-day cruises provided 150 samples for grain size analysis.

Presented here are tables with the station locations and types of data available followed by single sample summaries for each sample collected and analyzed. Included in each summary are location information, seafloor photographs, photographs of the sample (in field and laboratory) where available, collection information, sediment classifications, grain size statistics, and grain size distribution. Samples were analyzed with standard sieve and pipette analyses after Folk (1980). The sediment grain size classifications include: CMECS (Coastal and Marine Ecological Classification Standard; FGDC, 2012); Gradistat (Blot and Pye, 2001); and Wentworth (Wentworth, 1922; described in Folk, 1954, 1980). Statistics are based on the phi scale and include the graphic mean, sorting, skewness, and kurtosis (Folk, 1980). 


\section{Acknowledgements:}

The development of this database and an associated report "Surficial Geology of the Continental Shelf off New Hampshire, USA: Morphologic Features and Surficial Sediments" was supported by the University of New Hampshire/National Oceanic and Atmospheric Administration Joint Hydrographic Center Award Number NA15NOS4000200 and the Bureau of Ocean Energy Management Award Number M14AC00010.

\section{References:}

Blott, S.J. and Pye, K., 2001, Gradistat: A grain size distribution and statistics package for the analysis of unconsolidated sediments: Earth Surface Processes and Landforms, v. 26, no. 11, pp. 1237-1248. DOI: 10.1002/esp.261, Accessed March 1, 2020: http://www.kpal.co.uk/gradistat.html

FGDC (Federal Geographic Data Committee, Marine and Coastal Spatial Data Subcommittee), 2012, Coastal and Marine Ecological Classification Standard: FGDC-STD-018-2012, Washington, DC, 343 pp., https://www.fgdc.gov/standards/projects/cmecs-folder/CMECS_Version_06-2012_FINAL.pdf

Folk, R.L., 1954, The distinction between grain size and mineral composition in sedimentary-rock nomenclature: The Journal of Geology, vol. 62, number 4, pp. 344-359. DOI: 10.1086/626171

Folk, R.L., 1980, Petrology of sedimentary rocks: Hemphill Publishing Company, Austin, TX. 182 pp. Accessed May 2019, available online at https://repositories.lib.utexas.edu/handle/2152/22930

Ward, L.G., McAvoy, Z.S., Vallee-Anziani, M., and Morrison, R.C., 2021, Surficial Geology of the Continental Shelf off New Hampshire: Morphologic Features and Surficial Sediments: BOEM/New Hampshire Cooperative Agreement (Contract M14ACO0010) Technical Report, Department of Interior, Bureau of Ocean Energy Management, Marine Minerals Division, 45600 Woodland Road, Sterling, VA, 20166, 183 pp.

Wentworth, C., 1922, A scale of grade and class terms for clastic sediments: The Journal of Geology, v. 30, no. 5, pp. 377-392. Accessed May 2019, available online at https://www.jstor.org/stable/30063207 


\section{Station Locations and Sampling History}

\begin{tabular}{|c|c|c|c|c|c|c|c|}
\hline Station ID & $\begin{array}{l}\text { Latitude } \\
\text { WGS84 }\end{array}$ & $\begin{array}{c}\text { Longitude } \\
\text { WGS84 }\end{array}$ & $\begin{array}{c}\text { Water } \\
\text { Depth (m) }\end{array}$ & $\begin{array}{l}\text { Sample } \\
\text { Collected }\end{array}$ & $\begin{array}{c}\text { Video } \\
\text { Recorded }\end{array}$ & $\begin{array}{l}\text { Sample } \\
\text { Collected }\end{array}$ & $\begin{array}{l}\text { Analyzed } \\
\text { (Reported) }\end{array}$ \\
\hline GSH06_T03 & 43.066875 & -70.670733 & 11.0 & $8 / 1 / 2017$ & $Y$ & $Y$ & $Y$ \\
\hline GSH06_T04 & 43.071793 & -70.660163 & 15.2 & $8 / 1 / 2017$ & $\mathrm{Y}$ & $\mathrm{Y}$ & $\mathrm{Y}$ \\
\hline GSH06_T06 & 43.078768 & -70.656497 & 10.0 & $8 / 1 / 2017$ & $Y$ & & \\
\hline GSH06_T07 & 43.088600 & -70.653268 & 8.5 & $8 / 1 / 2017$ & $Y$ & $Y$ & $Y$ \\
\hline Shoals07_S01 & 42.872299 & -70.805992 & 9.4 & $10 / 13 / 2016$ & $Y$ & & \\
\hline Shoals07_S02 & 42.868036 & -70.810027 & 8.5 & $10 / 13 / 2016$ & $Y$ & $Y$ & $\mathrm{Y}$ \\
\hline Shoals07_S03 & 42.868160 & -70.803958 & 9.1 & $10 / 13 / 2016$ & $Y$ & & \\
\hline Shoals07_S04 & 42.864090 & -70.806587 & 14.6 & $10 / 13 / 2016$ & $Y$ & & \\
\hline Shoals07_S05 & 42.861377 & -70.806588 & 9.1 & $10 / 13 / 2016$ & $Y$ & & \\
\hline Shoals07_S06 & 42.858927 & -70.806217 & 15.8 & $10 / 13 / 2016$ & $Y$ & & \\
\hline Shoals07_S07 & 42.855850 & -70.807233 & 13.4 & $10 / 13 / 2016$ & $Y$ & $\mathrm{Y}$ & $\mathrm{Y}$ \\
\hline Shoals07_S08 & 42.855847 & -70.803396 & 16.8 & $10 / 13 / 2016$ & $Y$ & $Y$ & $\mathrm{Y}$ \\
\hline Shoals07_S09 & 42.854926 & -70.805300 & 12.0 & $10 / 6 / 2016$ & $Y$ & $\mathrm{Y}$ & $\mathrm{Y}$ \\
\hline Shoals07_S09-2 & 42.854691 & -70.805010 & 11.9 & $10 / 13 / 2016$ & $Y$ & & \\
\hline Shoals07_S11 & 42.849072 & -70.805784 & 13.4 & $10 / 6 / 2016$ & 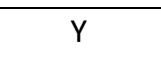 & $\mathrm{Y}$ & $\mathrm{Y}$ \\
\hline Shoals07_S11-2 & 42.849118 & -70.805506 & 12.2 & $10 / 13 / 2016$ & $Y$ & & \\
\hline Shoals07_S12 & 42.846389 & -70.805687 & 11.3 & $10 / 6 / 2016$ & $Y$ & & \\
\hline Shoals07_S13 & 42.843429 & -70.801355 & 15.2 & $10 / 6 / 2016$ & $Y$ & $\mathrm{Y}$ & $\mathrm{Y}$ \\
\hline Shoals07_S13-2 & 42.843243 & -70.801207 & 14.0 & $10 / 13 / 2016$ & $Y$ & & \\
\hline Shoals07_S14 & 42.829714 & -70.805199 & 8.8 & $10 / 6 / 2016$ & $Y$ & $Y$ & $\mathrm{Y}$ \\
\hline Shoals07_S14-3 & 42.829303 & -70.805617 & 7.6 & $10 / 13 / 2016$ & $Y$ & & \\
\hline GSH12_T01 & 43.061225 & -70.665870 & 20.1 & $8 / 1 / 2017$ & $Y$ & & \\
\hline GSH12_T02 & 43.065934 & -70.661847 & 16.8 & $8 / 1 / 2017$ & $Y$ & & \\
\hline GSH12_T03 & 43.062239 & -70.659768 & 14.9 & $8 / 1 / 2017$ & $\mathrm{Y}$ & & \\
\hline GSH12_T05 & 43.054176 & -70.655580 & 18.9 & $8 / 1 / 2017$ & $Y$ & & \\
\hline GSH12_T06 & 43.050548 & -70.655044 & 29.9 & $8 / 1 / 2017$ & $Y$ & $Y$ & $\mathrm{Y}$ \\
\hline GSH12_T08 & 43.065093 & -70.650458 & 12.8 & $8 / 1 / 2017$ & $\mathrm{Y}$ & & \\
\hline GSH12_T09 & 43.060070 & -70.648875 & 20.7 & $7 / 19 / 2017$ & $Y$ & & \\
\hline GSH12_T10 & 43.053340 & -70.643997 & 26.8 & $7 / 19 / 2017$ & $\mathrm{Y}$ & $\mathrm{Y}$ & $Y$ \\
\hline GSH12_T11 & 43.047794 & -70.642528 & 29.0 & $7 / 19 / 2017$ & $Y$ & & \\
\hline GSH12_T12 & 43.044385 & -70.635119 & 32.0 & $7 / 19 / 2017$ & $Y$ & $Y$ & $Y$ \\
\hline
\end{tabular}


Station Locations and Sampling History: Continued

\begin{tabular}{|c|c|c|c|c|c|c|c|}
\hline Station ID & $\begin{array}{l}\text { Latitude } \\
\text { WGS84 }\end{array}$ & $\begin{array}{l}\text { Longitude } \\
\text { WGS84 }\end{array}$ & $\begin{array}{c}\text { Water } \\
\text { Depth }(m)\end{array}$ & $\begin{array}{c}\text { Sample } \\
\text { Collected }\end{array}$ & $\begin{array}{c}\text { Video } \\
\text { Recorded }\end{array}$ & $\begin{array}{c}\text { Sample } \\
\text { Collected }\end{array}$ & $\begin{array}{c}\text { Analyzed } \\
\text { (Reported) }\end{array}$ \\
\hline GSH12_T13 & 43.074722 & -70.651201 & 10.7 & $7 / 19 / 2017$ & $Y$ & & \\
\hline GSH12_T14 & 43.068737 & -70.643519 & 16.8 & $8 / 1 / 2017$ & $Y$ & & \\
\hline GSH12_T15 & 43.063468 & -70.640147 & 14.6 & $8 / 1 / 2017$ & $Y$ & & \\
\hline GSH12_T16 & 43.050815 & -70.631221 & 20.4 & $7 / 19 / 2017$ & $Y$ & & \\
\hline GSH12_T17 & 43.062383 & -70.631459 & 26.5 & $7 / 19 / 2017$ & $Y$ & $Y$ & $Y$ \\
\hline GSH12_T18 & 43.078743 & -70.645144 & 15.2 & $8 / 1 / 2017$ & $Y$ & $Y$ & $\mathrm{Y}$ \\
\hline GSH12_T19 & 43.082730 & -70.646555 & 10.1 & $8 / 1 / 2017$ & $Y$ & & \\
\hline GSH12_T20 & 43.082758 & -70.650398 & 14.3 & $8 / 1 / 2017$ & $Y$ & $Y$ & $Y$ \\
\hline GSH12_T21 & 43.088377 & -70.645729 & 18.3 & $8 / 1 / 2017$ & $Y$ & $Y$ & $Y$ \\
\hline GSH12_T22 & 43.091586 & -70.650512 & 9.4 & $7 / 19 / 2017$ & $Y$ & $Y$ & $Y$ \\
\hline GSH12_T23 & 43.095915 & -70.645111 & 12.2 & $7 / 19 / 2017$ & $Y$ & $Y$ & $Y$ \\
\hline GSH12_T24 & 43.089942 & -70.655696 & 7.3 & $8 / 1 / 2017$ & $Y$ & $Y$ & $\mathrm{Y}$ \\
\hline GSH12_T50 & 43.055388 & -70.628376 & 30.5 & $7 / 19 / 2017$ & $Y$ & & \\
\hline GSH12_T51 & 43.055253 & -70.623333 & 33.2 & $7 / 19 / 2017$ & $Y$ & $Y$ & $Y$ \\
\hline GSH12_T52 & 43.056485 & -70.632533 & 18.3 & $7 / 19 / 2017$ & $Y$ & & \\
\hline SH12-01-A & 43.048528 & -70.649317 & 27.1 & $6 / 28 / 2012$ & $Y$ & $Y$ & \\
\hline SH12-02 & 43.053003 & -70.644819 & 23.5 & $6 / 28 / 2012$ & $Y$ & $Y$ & $Y$ \\
\hline SH12-03-A & 43.052022 & -70.641728 & 17.1 & $6 / 28 / 2012$ & $Y$ & & \\
\hline SH12-03-B & 43.051731 & -70.641808 & 17.1 & $6 / 28 / 2012$ & $Y$ & & \\
\hline SH12-03-C & 43.052122 & -70.641561 & $\mathrm{~N} / \mathrm{A}$ & $6 / 28 / 2012$ & $Y$ & & \\
\hline SH12-04 & 43.048156 & -70.639286 & 24.4 & $6 / 28 / 2012$ & $Y$ & $Y$ & $Y$ \\
\hline SH12-05 & 43.043894 & -70.634703 & 32.6 & $6 / 28 / 2012$ & $Y$ & $Y$ & $Y$ \\
\hline SH12-06-A & 43.050539 & -70.632264 & 21.6 & $6 / 28 / 2012$ & $Y$ & & \\
\hline SH12-06-B & 43.050553 & -70.632428 & $\mathrm{~N} / \mathrm{A}$ & $6 / 28 / 2012$ & $Y$ & & \\
\hline SH12-07 & 43.050233 & -70.629839 & $\mathrm{~N} / \mathrm{A}$ & $6 / 28 / 2012$ & $Y$ & & \\
\hline SH12-08 & 43.052706 & -70.624306 & 33.2 & $6 / 28 / 2012$ & $Y$ & $Y$ & \\
\hline SH12-09 & 43.055544 & -70.623825 & 33.5 & $6 / 28 / 2012$ & $Y$ & $Y$ & $Y$ \\
\hline SH12-10 & 43.061981 & -70.649350 & N/A & $6 / 28 / 2012$ & $Y$ & $Y$ & \\
\hline SH12-11 & 43.057017 & -70.650367 & N/A & $6 / 28 / 2012$ & $Y$ & $Y$ & $Y$ \\
\hline SH12-12 & 43.094919 & -70.639372 & N/A & $6 / 28 / 2012$ & $Y$ & $Y$ & $Y$ \\
\hline SH12-13 & 43.097294 & -70.648036 & 10.1 & $6 / 28 / 2012$ & $Y$ & $Y$ & $Y$ \\
\hline SH12-14 & 43.096169 & -70.646881 & 11.3 & $6 / 28 / 2012$ & $Y$ & $Y$ & $Y$ \\
\hline SH12-15-A & 43.093869 & -70.648525 & 8.2 & $6 / 28 / 2012$ & $Y$ & & \\
\hline SH12-15-B & 43.093020 & -70.647627 & 13.7 & $6 / 28 / 2012$ & $Y$ & $Y$ & $Y$ \\
\hline SH12-16 & 43.091936 & -70.651581 & 8.8 & $6 / 28 / 2012$ & $Y$ & $Y$ & $Y$ \\
\hline SH12-17 & 43.063233 & -70.663283 & 22.6 & $6 / 28 / 2012$ & $Y$ & $Y$ & $Y$ \\
\hline
\end{tabular}


Station Locations and Sampling History: Continued

\begin{tabular}{|c|c|c|c|c|c|c|c|}
\hline Station ID & $\begin{array}{l}\text { Latitude } \\
\text { WGS84 }\end{array}$ & $\begin{array}{l}\text { Longitude } \\
\text { WGS84 }\end{array}$ & $\begin{array}{c}\text { Water } \\
\text { Depth }(m)\end{array}$ & $\begin{array}{c}\text { Sample } \\
\text { Collected }\end{array}$ & $\begin{array}{c}\text { Video } \\
\text { Recorded }\end{array}$ & $\begin{array}{l}\text { Sample } \\
\text { Collected }\end{array}$ & $\begin{array}{c}\text { Analyzed } \\
\text { (Reported) }\end{array}$ \\
\hline GSH13-T10 & 43.026381 & -70.714468 & 11.0 & $7 / 17 / 2017$ & $Y$ & & \\
\hline GSH13-T11 & 43.024318 & -70.713641 & 13.4 & $7 / 17 / 2017$ & $Y$ & $Y$ & $Y$ \\
\hline GSH13-T13 & 43.022075 & -70.719898 & 11.0 & $7 / 17 / 2017$ & $Y$ & $Y$ & $Y$ \\
\hline GSH13-T14 & 43.020354 & -70.722841 & 9.4 & $7 / 17 / 2017$ & $Y$ & $\mathrm{Y}$ & $Y$ \\
\hline GSH13-T15 & 43.017187 & -70.719325 & 15.5 & $7 / 17 / 2017$ & $\mathrm{Y}$ & $Y$ & $\mathrm{Y}$ \\
\hline GSH13-T16 & 43.014625 & -70.722427 & 13.4 & $7 / 17 / 2017$ & $\mathrm{Y}$ & & \\
\hline GSH13-T17 & 43.014386 & -70.726680 & 8.5 & $7 / 17 / 2017$ & $Y$ & $Y$ & $\mathrm{Y}$ \\
\hline GSH14_T12 & 42.992188 & -70.726966 & 17.1 & $7 / 18 / 2017$ & $\mathrm{Y}$ & & \\
\hline GSH14_T14 & 42.983649 & -70.738158 & 15.8 & $7 / 18 / 2017$ & $Y$ & $\mathrm{Y}$ & $\mathrm{Y}$ \\
\hline GSH14_T15 & 42.982267 & -70.736127 & 18.9 & $7 / 18 / 2017$ & $Y$ & $Y$ & $\mathrm{Y}$ \\
\hline GSH14_T16 & 42.978441 & -70.736979 & 19.8 & $7 / 18 / 2017$ & $Y$ & $Y$ & $Y$ \\
\hline GSH14_T17 & 42.977165 & -70.740457 & 19.5 & $11 / 1 / 2016$ & $Y$ & $\mathrm{Y}$ & $\mathrm{Y}$ \\
\hline GSH14_T18 & 42.974451 & -70.743121 & 17.4 & $11 / 1 / 2016$ & $Y$ & & \\
\hline GSH14_T19 & 42.972294 & -70.745278 & 16.2 & $11 / 1 / 2016$ & $Y$ & & \\
\hline GSH14_T20 & 42.974777 & -70.749365 & 17.4 & $11 / 1 / 2016$ & $Y$ & $\mathrm{Y}$ & $\mathrm{Y}$ \\
\hline GSH14_T21 & 42.974018 & -70.750086 & 15.8 & $7 / 18 / 2017$ & $Y$ & & \\
\hline GSH14_T23 & 42.978113 & -70.746629 & 15.8 & $11 / 1 / 2016$ & $Y$ & & \\
\hline GSH14_T24 & 42.979850 & -70.745290 & 14.9 & $7 / 18 / 2017$ & $Y$ & $Y$ & $Y$ \\
\hline GSH14_T25 & 42.981999 & -70.745452 & 15.2 & $11 / 1 / 2016$ & $Y$ & & \\
\hline GSH14_T27 & 42.986960 & -70.743435 & 12.2 & $7 / 18 / 2017$ & $Y$ & & \\
\hline GSH14_T35 & 42.979467 & -70.742292 & 16.5 & $7 / 18 / 2017$ & $Y$ & $\mathrm{Y}$ & $\mathrm{Y}$ \\
\hline GSH14_T36 & 42.979925 & -70.752487 & 8.2 & $7 / 18 / 2017$ & $Y$ & $Y$ & $\mathrm{Y}$ \\
\hline SH14_BS-01A & 42.972965 & -70.743245 & 15.8 & $6 / 30 / 2014$ & $Y$ & & \\
\hline SH14_BS-02 & 42.979475 & -70.741855 & 18.3 & $6 / 30 / 2014$ & $Y$ & $Y$ & $\mathrm{Y}$ \\
\hline SH14_BS-Extra & 42.983121 & -70.752974 & 8.1 & $6 / 30 / 2014$ & $Y$ & $\mathrm{Y}$ & \\
\hline SH14_BS-03A & 42.983972 & -70.736481 & 20.3 & $6 / 30 / 2014$ & $\mathrm{Y}$ & $\mathrm{Y}$ & $\mathrm{Y}$ \\
\hline SH14_BS-03B & 42.983936 & -70.736288 & 20.9 & $6 / 30 / 2014$ & $Y$ & $Y$ & $\mathrm{Y}$ \\
\hline SH14_BS-04A & 42.990993 & -70.731050 & 16.9 & $6 / 30 / 2014$ & $\mathrm{Y}$ & $\mathrm{Y}$ & \\
\hline SH14_BS-04B & 42.991207 & -70.731278 & 16.8 & $6 / 30 / 2014$ & $\mathrm{Y}$ & & \\
\hline SH14_BS-05 & 42.996231 & -70.740064 & 10.6 & $6 / 30 / 2014$ & $\mathrm{Y}$ & $\mathrm{Y}$ & $\mathrm{Y}$ \\
\hline SH14_BS-06 & 42.995856 & -70.728489 & 17.7 & $7 / 1 / 2014$ & $\mathrm{Y}$ & $\mathrm{Y}$ & $\mathrm{Y}$ \\
\hline SH14_BS-07 & 43.000470 & -70.727247 & 16.5 & $7 / 1 / 2014$ & $Y$ & $\mathrm{Y}$ & $Y$ \\
\hline SH14_BS-08 & 43.000737 & -70.722898 & 19.8 & $7 / 1 / 2014$ & $Y$ & $Y$ & $Y$ \\
\hline SH14_BS-09 & 43.006318 & -70.720523 & 8.8 & $7 / 1 / 2014$ & $\mathrm{Y}$ & $\mathrm{Y}$ & $\mathrm{Y}$ \\
\hline SH14_BS-10 & 43.008803 & -70.714451 & 20.7 & $7 / 1 / 2014$ & $Y$ & $\mathrm{Y}$ & $Y$ \\
\hline SH14_BS-11 & 42.996592 & -70.727623 & 17.4 & $7 / 1 / 2014$ & $\mathrm{Y}$ & $\mathrm{Y}$ & \\
\hline GSH15_T01 & 42.930871 & -70.790805 & 7.6 & $11 / 1 / 2016$ & $\mathrm{Y}$ & $\mathrm{Y}$ & $\mathrm{Y}$ \\
\hline
\end{tabular}


Station Locations and Sampling History: Continued

\begin{tabular}{|c|c|c|c|c|c|c|c|}
\hline Station ID & $\begin{array}{l}\text { Latitude } \\
\text { WGS84 }\end{array}$ & $\begin{array}{l}\text { Longitude } \\
\text { WGS84 }\end{array}$ & $\begin{array}{c}\text { Water } \\
\text { Depth }(\mathrm{m})\end{array}$ & $\begin{array}{l}\text { Sample } \\
\text { Collected }\end{array}$ & $\begin{array}{c}\text { Video } \\
\text { Recorded }\end{array}$ & $\begin{array}{l}\text { Sample } \\
\text { Collected }\end{array}$ & $\begin{array}{c}\text { Analyzed } \\
\text { (Reported) }\end{array}$ \\
\hline GSH15_T02 & 42.926577 & -70.790042 & 8.8 & $11 / 1 / 2016$ & $Y$ & $Y$ & $Y$ \\
\hline GSH15_T04 & 42.943020 & -70.777881 & 14.3 & $11 / 1 / 2016$ & $Y$ & & \\
\hline GSH15_T05 & 42.947430 & -70.775635 & 13.1 & $11 / 1 / 2016$ & $Y$ & $Y$ & $Y$ \\
\hline GSH15_T06 & 42.941297 & -70.771655 & 15.2 & $11 / 1 / 2016$ & $Y$ & & \\
\hline GSH15_T07 & 42.935006 & -70.767757 & 21.9 & $10 / 6 / 2016$ & $Y$ & $\mathrm{Y}$ & $Y$ \\
\hline GSH15_T08 & 42.932906 & -70.773033 & 20.1 & $11 / 1 / 2016$ & $Y$ & $Y$ & $Y$ \\
\hline GSH15_T09 & 42.927505 & -70.777355 & 18.3 & $11 / 1 / 2016$ & $Y$ & & \\
\hline GSH15_T10 & 42.922464 & -70.780157 & 15.8 & $10 / 6 / 2016$ & $Y$ & $Y$ & $Y$ \\
\hline GSH15_T12 & 42.924009 & -70.786317 & 12.2 & $11 / 1 / 2016$ & $Y$ & & \\
\hline GSH15_T14 & 42.944763 & -70.761670 & 23.5 & 11/1/2016 & Y & $Y$ & Y \\
\hline GSH15_T15 & 42.941103 & -70.762389 & 82.0 & $11 / 1 / 2016$ & $Y$ & $Y$ & $Y$ \\
\hline GSH15_T17 & 42.951865 & -70.764080 & 14.3 & $11 / 1 / 2016$ & $Y$ & & \\
\hline GSH15_T18 & 42.957298 & -70.767873 & 9.8 & $11 / 1 / 2016$ & $Y$ & & \\
\hline GSH15_T19 & 42.955194 & -70.761460 & 12.8 & $11 / 1 / 2016$ & $Y$ & & \\
\hline GSH16-T01 & 43.045297 & -70.697871 & 16.8 & $7 / 10 / 2017$ & $Y$ & $Y$ & $Y$ \\
\hline GSH16-T02 & 43.042899 & -70.697748 & 17.1 & $7 / 10 / 2017$ & $Y$ & $Y$ & $\bar{Y}$ \\
\hline GSH16-T03 & 43.041998 & -70.694267 & 16.2 & 7/10/2017 & $Y$ & $Y$ & $Y$ \\
\hline GSH16-T04 & 43.039079 & -70.686425 & 21.3 & $7 / 10 / 2017$ & $Y$ & $Y$ & $Y$ \\
\hline GSH16-T05 & 43.035313 & -70.683228 & 25.9 & $7 / 10 / 2017$ & $Y$ & $Y$ & $Y$ \\
\hline GSH16-T06 & 43.031908 & -70.682273 & 27.7 & $7 / 10 / 2017$ & $Y$ & $Y$ & $Y$ \\
\hline GSH16-T07 & 43.026767 & -70.677968 & 25.9 & $7 / 11 / 2017$ & $Y$ & $Y$ & $Y$ \\
\hline GSH16-T08 & 43.022048 & -70.682323 & 23.5 & 7/11/2017 & $Y$ & & \\
\hline GSH16-T09 & 43.018776 & -70.687632 & 18.3 & $7 / 11 / 2017$ & $\mathrm{Y}$ & & \\
\hline GSH16-T10 & 43.021826 & -70.702667 & 8.8 & $7 / 11 / 2017$ & $Y$ & & \\
\hline GSH16-T11 & 43.024464 & -70.704939 & 18.9 & $7 / 11 / 2017$ & $Y$ & $Y$ & $Y$ \\
\hline GSH16-T12 & 43.025693 & -70.710856 & 15.8 & $7 / 11 / 2017$ & $Y$ & $Y$ & $Y$ \\
\hline GSH16-T13 & 43.030201 & -70.708662 & 16.5 & $7 / 11 / 2017$ & $Y$ & & \\
\hline GSH16-T14 & 43.030153 & -70.702878 & 17.7 & $7 / 11 / 2017$ & $Y$ & $\mathrm{Y}$ & $Y$ \\
\hline GSH16-T15 & 43.031050 & -70.698484 & 17.7 & $7 / 11 / 2017$ & $Y$ & $Y$ & $Y$ \\
\hline GSH16-T16 & 43.033065 & -70.698736 & 16.8 & $7 / 11 / 2017$ & $Y$ & & \\
\hline GSH16-T17 & 43.033956 & -70.693845 & 18.3 & $7 / 11 / 2017$ & $Y$ & $Y$ & $Y$ \\
\hline GSH16-T18 & 43.036400 & -70.695581 & 16.8 & $7 / 11 / 2017$ & $Y$ & $Y$ & $Y$ \\
\hline GSH16-TS06 & 42.855997 & -70.794752 & 18.0 & $10 / 13 / 2016$ & $Y$ & $Y$ & $Y$ \\
\hline GSH16-TSO9 & 42.857124 & -70.799412 & 16.5 & $10 / 13 / 2016$ & $Y$ & $Y$ & $Y$ \\
\hline GSH16-TS10 & 42.853463 & -70.799742 & 17.4 & $10 / 13 / 2016$ & $\mathrm{Y}$ & $Y$ & $\mathrm{Y}$ \\
\hline SH18-T01 & 43.110880 & 70.621355 & 25.0 & $7 / 9 / 2018$ & $Y$ & $Y$ & $Y$ \\
\hline SH18-T02 & 43.111675 & 70.624758 & 18.2 & $7 / 9 / 2018$ & $Y$ & $Y$ & \\
\hline
\end{tabular}


Station Locations and Sampling History: Continued

\begin{tabular}{|c|c|c|c|c|c|c|c|}
\hline Station ID & $\begin{array}{l}\text { Latitude } \\
\text { WGS84 }\end{array}$ & $\begin{array}{c}\text { Longitude } \\
\text { WGS84 }\end{array}$ & $\begin{array}{c}\text { Water } \\
\text { Depth }(\mathrm{m})\end{array}$ & $\begin{array}{l}\text { Sample } \\
\text { Collected }\end{array}$ & $\begin{array}{c}\text { Video } \\
\text { Recorded }\end{array}$ & $\begin{array}{l}\text { Sample } \\
\text { Collected }\end{array}$ & $\begin{array}{c}\text { Analyzed } \\
\text { (Reported) }\end{array}$ \\
\hline SH18-T03 & 43.112021 & 70.617811 & 22.5 & $7 / 9 / 2018$ & $Y$ & $\mathrm{Y}$ & \\
\hline SH18-T04 & 43.115655 & 70.625815 & 13.6 & $7 / 9 / 2018$ & $Y$ & $\mathrm{Y}$ & \\
\hline SH18-T05 & 43.097957 & 70.635421 & 14.7 & $7 / 9 / 2018$ & $Y$ & $\mathrm{Y}$ & \\
\hline SH18-T06 & 43.098228 & 70.630833 & 14.7 & $7 / 9 / 2018$ & $Y$ & $\mathrm{Y}$ & \\
\hline SH18-T07 & 43.100331 & 70.629278 & 21.3 & $7 / 9 / 2018$ & $Y$ & $\bar{Y}$ & $Y$ \\
\hline SH18-T08 & 43.098002 & 70.625083 & 22.3 & $7 / 9 / 2018$ & $Y$ & & \\
\hline SH18-T09 & 43.095290 & 70.627049 & 20.0 & $7 / 9 / 2018$ & $Y$ & $Y$ & $Y$ \\
\hline SH18-T10 & 43.085579 & 70.636676 & 27.1 & $7 / 9 / 2018$ & $Y$ & $\mathrm{Y}$ & $Y$ \\
\hline SH18-T11 & 43.078903 & 70.640364 & 13.9 & $7 / 9 / 2018$ & $Y$ & $\mathrm{Y}$ & \\
\hline SH18-T12 & 43.075575 & 70.638538 & 10.3 & $7 / 9 / 2018$ & $Y$ & & \\
\hline NSBE-T01 & 42.968322 & -70.660788 & 43.6 & $8 / 8 / 2017$ & $Y$ & $Y$ & $Y$ \\
\hline NSBE-T02 & 42.971764 & -70.653901 & 40.5 & $8 / 8 / 2017$ & $Y$ & $Y$ & $Y$ \\
\hline NSBE-T03 & 42.976383 & -70.643870 & 35.7 & $8 / 7 / 2017$ & $Y$ & $\mathrm{Y}$ & $Y$ \\
\hline NSBE-T04 & 42.990159 & -70.638573 & 20.7 & $8 / 7 / 2017$ & $Y$ & $\mathrm{Y}$ & $Y$ \\
\hline NSBE-T05 & 42.986699 & -70.645589 & 24.4 & $8 / 7 / 2017$ & $\bar{Y}$ & $\mathrm{Y}$ & $\mathrm{Y}$ \\
\hline NSBE-T06 & 42.981125 & -70.655257 & 26.8 & $8 / 7 / 2017$ & $Y$ & & \\
\hline NSBE-T06a & 42.980573 & -70.655550 & 27.1 & $8 / 7 / 2017$ & $Y$ & $Y$ & $Y$ \\
\hline NSBE-T07 & 42.977264 & -70.669788 & 24.7 & $8 / 7 / 2017$ & $Y$ & & \\
\hline NSBE-T08 & 42.980381 & -70.672999 & 21.0 & $8 / 7 / 2017$ & $Y$ & & \\
\hline NSBE-T09a & 42.981201 & -70.677443 & 21.9 & $8 / 7 / 2017$ & $Y$ & & \\
\hline NSBE-T10 & 42.976370 & -70.678328 & 37.5 & $8 / 7 / 2017$ & $\mathrm{Y}$ & $\mathrm{Y}$ & $Y$ \\
\hline NSBE-T11 & 42.981649 & -70.686066 & 30.8 & $8 / 8 / 2017$ & $Y$ & & \\
\hline NSBE-T12 & 42.984786 & -70.685358 & 32.3 & $8 / 8 / 2017$ & $Y$ & $Y$ & $\mathrm{Y}$ \\
\hline NSBE-T13 & 42.987658 & -70.660873 & 30.5 & $8 / 8 / 2017$ & $Y$ & $Y$ & $\mathrm{Y}$ \\
\hline NSBE-T14 & 42.990282 & -70.668139 & 26.8 & $8 / 8 / 2017$ & $Y$ & $\mathrm{Y}$ & $Y$ \\
\hline NSBE-T15 & 42.991793 & -70.674793 & 29.9 & $8 / 8 / 2017$ & $Y$ & $\mathrm{Y}$ & $Y$ \\
\hline NSBE-T16 & 42.996550 & -70.670892 & 33.5 & $8 / 8 / 2017$ & $Y$ & & \\
\hline NSBE-T17 & 42.991445 & -70.689473 & 19.5 & $8 / 16 / 2017$ & $Y$ & & \\
\hline NSBE-T18 & 42.990468 & -70.695505 & 17.4 & $8 / 16 / 2017$ & $Y$ & & \\
\hline NSBE-T19 & 42.989167 & -70.699695 & 21.3 & $8 / 8 / 2017$ & $Y$ & & \\
\hline NSBE-T20 & 42.987371 & -70.694519 & 25.3 & $8 / 8 / 2017$ & $Y$ & $Y$ & $Y$ \\
\hline NSBE-T21a & 42.991405 & -70.702695 & 27.4 & $8 / 8 / 2017$ & $Y$ & $Y$ & $Y$ \\
\hline NSBE-T22 & 43.001453 & -70.657417 & 19.2 & $8 / 15 / 2017$ & $Y$ & & \\
\hline NSBE-T23 & 43.005541 & -70.668076 & 17.1 & $8 / 15 / 2017$ & $Y$ & & \\
\hline NSBE-T24 & 43.011734 & -70.676721 & 18.9 & $8 / 16 / 2017$ & $Y$ & & \\
\hline NSBE-T25 & 43.009708 & -70.664556 & 27.7 & $8 / 15 / 2017$ & $Y$ & & \\
\hline NSBE-T26 & 43.003448 & -70.672623 & 26.8 & $8 / 15 / 2017$ & $\mathrm{Y}$ & & \\
\hline NSBE-T27 & 43.015685 & -70.676396 & 27.4 & $8 / 16 / 2017$ & $Y$ & & \\
\hline
\end{tabular}


Station Locations and Sampling History: Continued

\begin{tabular}{|c|c|c|c|c|c|c|c|}
\hline Station ID & $\begin{array}{c}\text { Latitude } \\
\text { WGS84 }\end{array}$ & $\begin{array}{c}\text { Longitude } \\
\text { WGS84 }\end{array}$ & $\begin{array}{c}\text { Water } \\
\text { Depth }(\mathrm{m})\end{array}$ & $\begin{array}{c}\text { Sample } \\
\text { Collected }\end{array}$ & $\begin{array}{c}\text { Video } \\
\text { Recorded }\end{array}$ & $\begin{array}{c}\text { Sample } \\
\text { Collected }\end{array}$ & $\begin{array}{c}\text { Analyzed } \\
\text { (Reported) }\end{array}$ \\
\hline NSBE-T28 & 42.978978 & -70.674840 & 19.8 & $8 / 15 / 2017$ & $Y$ & & \\
\hline NSBE-T29 & 42.979621 & -70.679554 & 26.5 & $8 / 15 / 2017$ & $Y$ & $Y$ & $Y$ \\
\hline NSBE-T30 & 42.985811 & -70.671644 & 26.2 & $8 / 15 / 2017$ & $Y$ & $Y$ & $Y$ \\
\hline NSBE-T31 & 42.987403 & -70.669282 & 33.5 & $8 / 15 / 2017$ & $Y$ & & \\
\hline NSBE-T32 & 42.985106 & -70.663309 & 34.7 & $8 / 15 / 2017$ & $\mathrm{Y}$ & $\mathrm{Y}$ & $Y$ \\
\hline NSBE-T33 & 42.984001 & -70.673906 & 20.1 & $8 / 15 / 2017$ & $Y$ & & \\
\hline NSBE-T34 & 42.982058 & -70.702832 & 18.9 & $8 / 16 / 2017$ & $Y$ & & \\
\hline NSBE-T35 & 42.990936 & -70.712882 & 13.7 & $8 / 16 / 2017$ & $\mathrm{Y}$ & & \\
\hline NSBE-T36 & 42.995784 & -70.710278 & 25.6 & $8 / 16 / 2017$ & $Y$ & $Y$ & $Y$ \\
\hline NSBE-T37 & 42.997882 & -70.688657 & 14.3 & $8 / 16 / 2017$ & $Y$ & & \\
\hline NSBE-T38 & 43.000245 & -70.701154 & 14.6 & $8 / 16 / 2017$ & $Y$ & & \\
\hline NSBE-T39 & 43.005076 & -70.694332 & 23.5 & $8 / 16 / 2017$ & $\mathrm{Y}$ & $Y$ & $Y$ \\
\hline NSBE-T40 & 42.973863 & -70.698420 & 32.9 & $8 / 16 / 2017$ & $Y$ & $Y$ & $Y$ \\
\hline NSBE-T41 & 42.983621 & -70.710607 & 16.8 & $8 / 16 / 2017$ & $Y$ & & \\
\hline NSBE-T42 & 42.971760 & -70.670194 & 37.2 & $8 / 16 / 2017$ & $Y$ & $Y$ & $Y$ \\
\hline NSBE-T43 & 43.007699 & -70.664900 & 27.4 & $8 / 15 / 2017$ & $\mathrm{Y}$ & & \\
\hline NSBE-T45 & 43.026969 & -70.648673 & 33.5 & $8 / 16 / 2017$ & $Y$ & $Y$ & $Y$ \\
\hline NSBE-T47 & 43.006194 & -70.679682 & 26.8 & $8 / 16 / 2017$ & $Y$ & & \\
\hline NSBE-T52 & 43.022767 & -70.666995 & 21.6 & $8 / 16 / 2017$ & $\mathrm{Y}$ & & \\
\hline NSBE-T59 & 42.998502 & -70.648205 & 27.4 & $8 / 15 / 2017$ & $Y$ & & \\
\hline NSBE-T60 & 42.995995 & -70.652881 & 21.9 & $8 / 15 / 2017$ & $Y$ & & \\
\hline NSBE-T61 & 42.992317 & -70.659907 & 30.2 & $8 / 16 / 2017$ & $Y$ & $Y$ & $Y$ \\
\hline NSBE-T62 & 42.985456 & -70.653036 & 23.8 & $8 / 15 / 2017$ & $Y$ & $Y$ & $Y$ \\
\hline NSBE-T64 & 42.978964 & -70.663495 & 25.6 & $8 / 15 / 2017$ & $Y$ & $Y$ & $Y$ \\
\hline
\end{tabular}




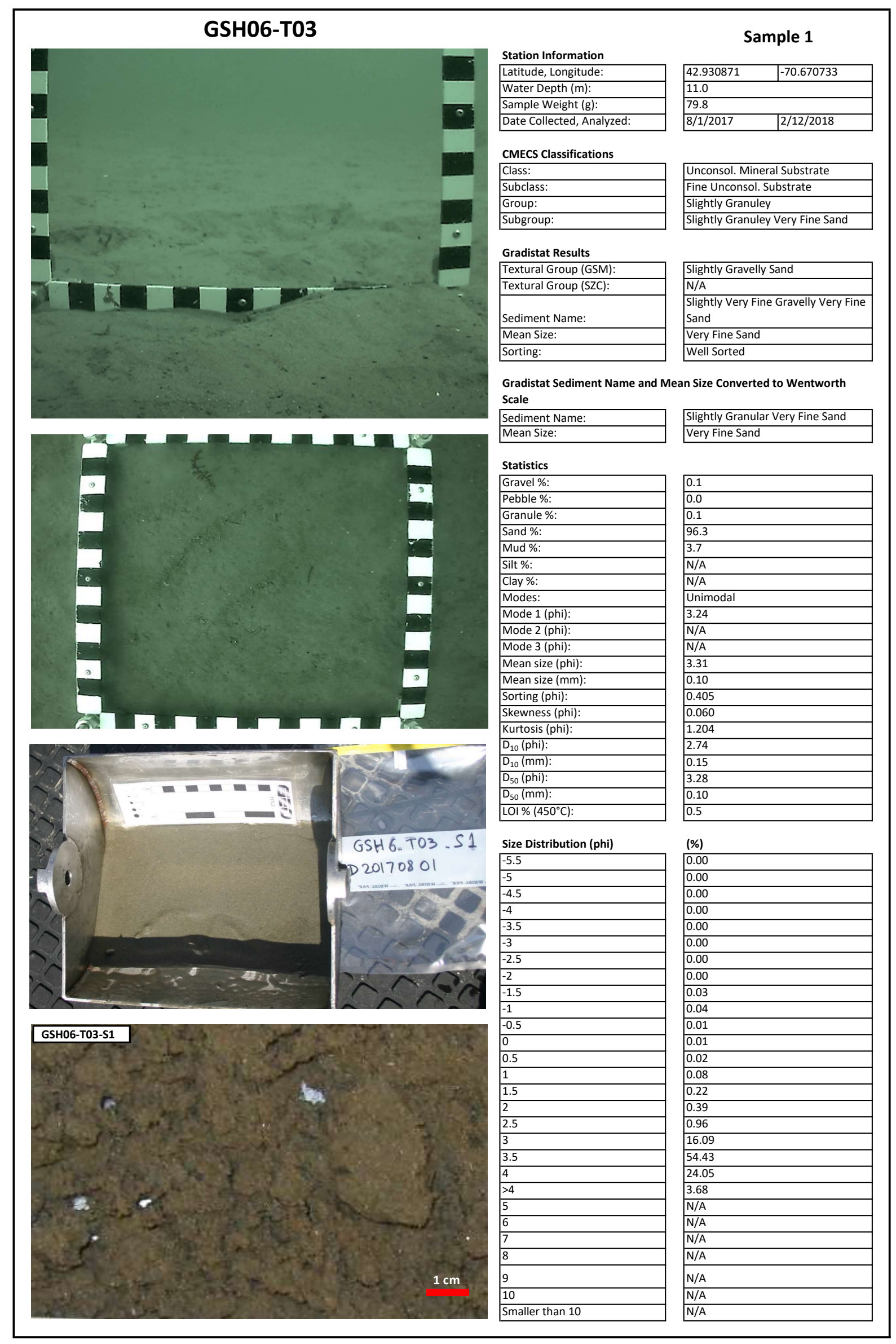




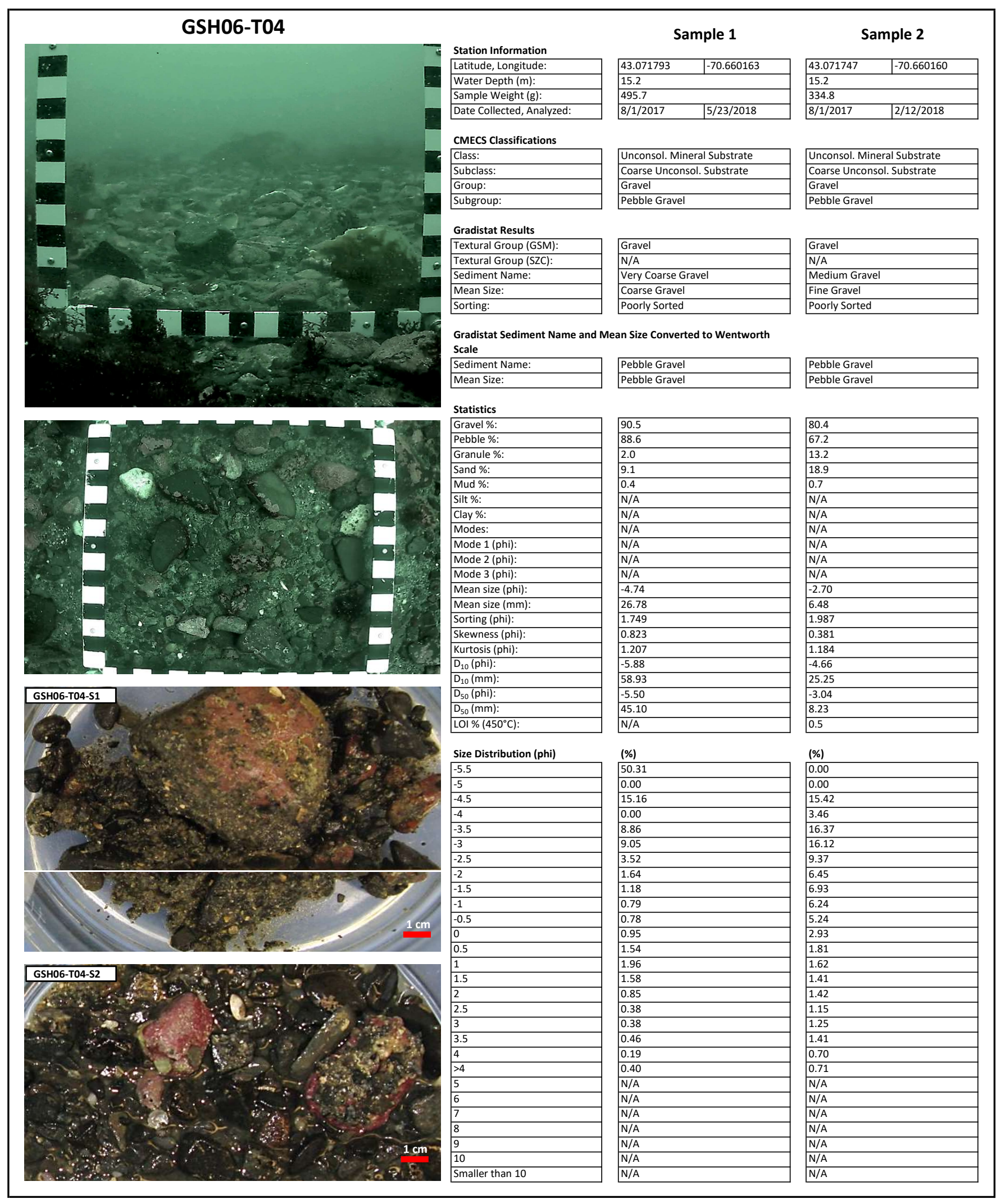




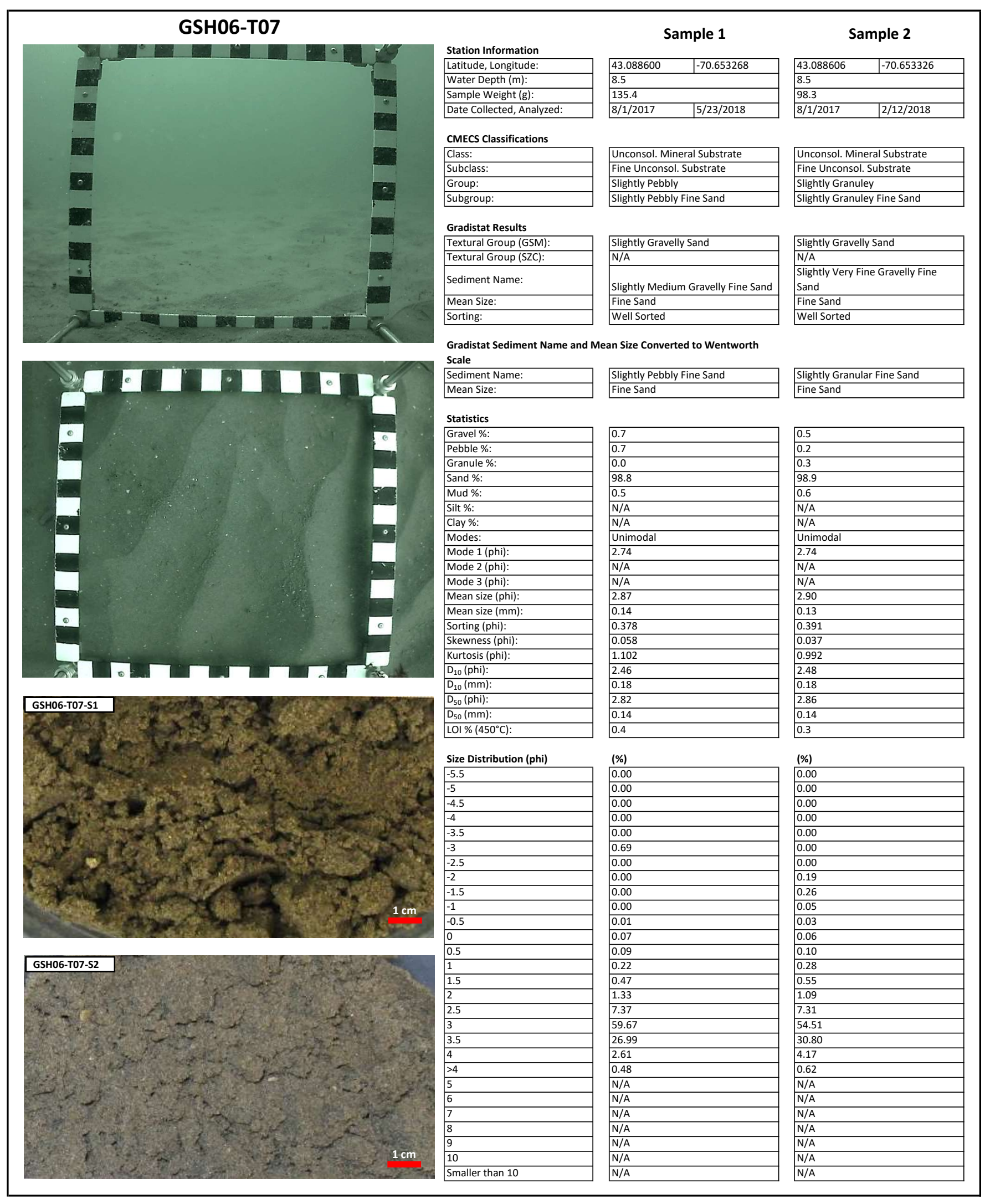




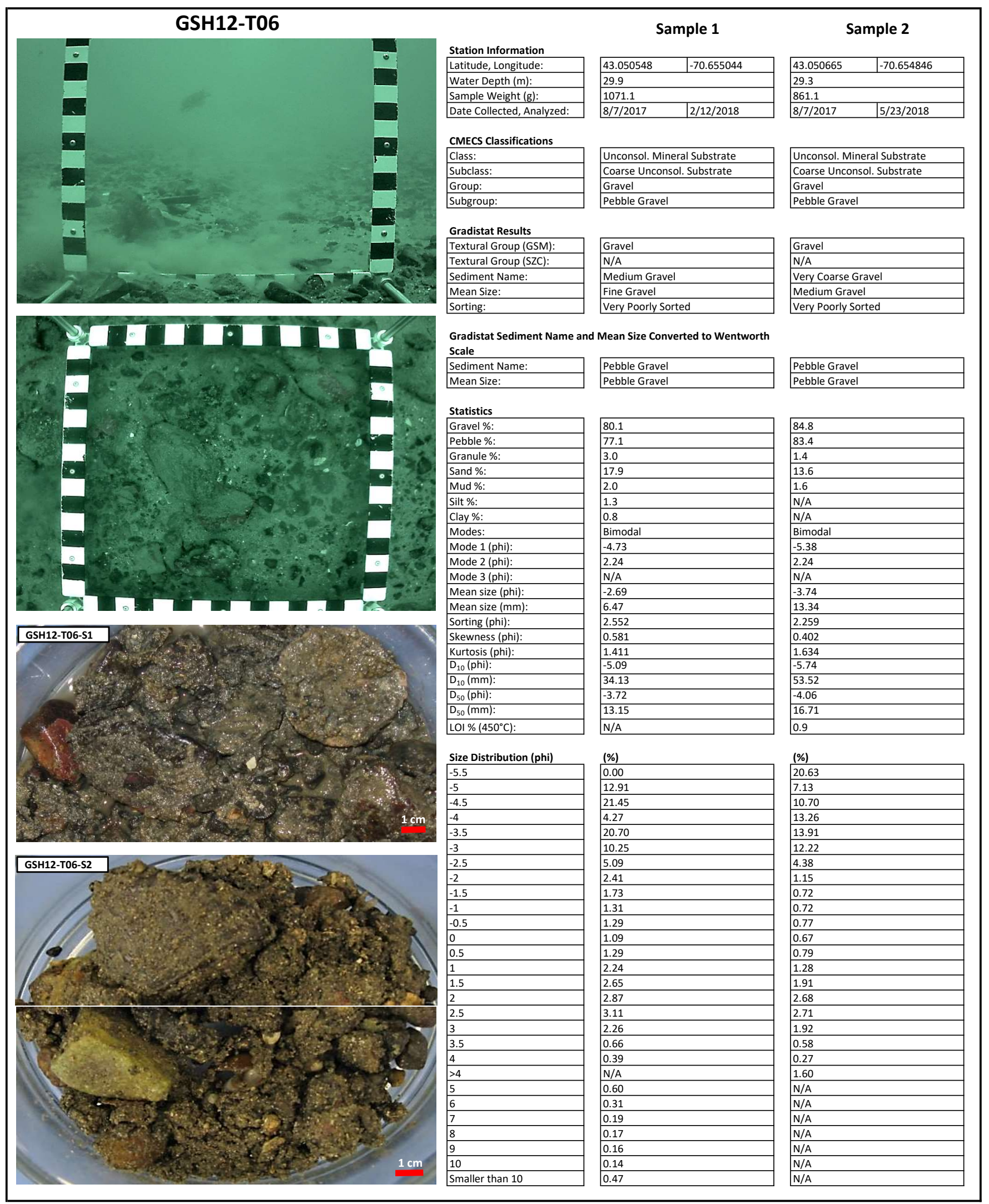




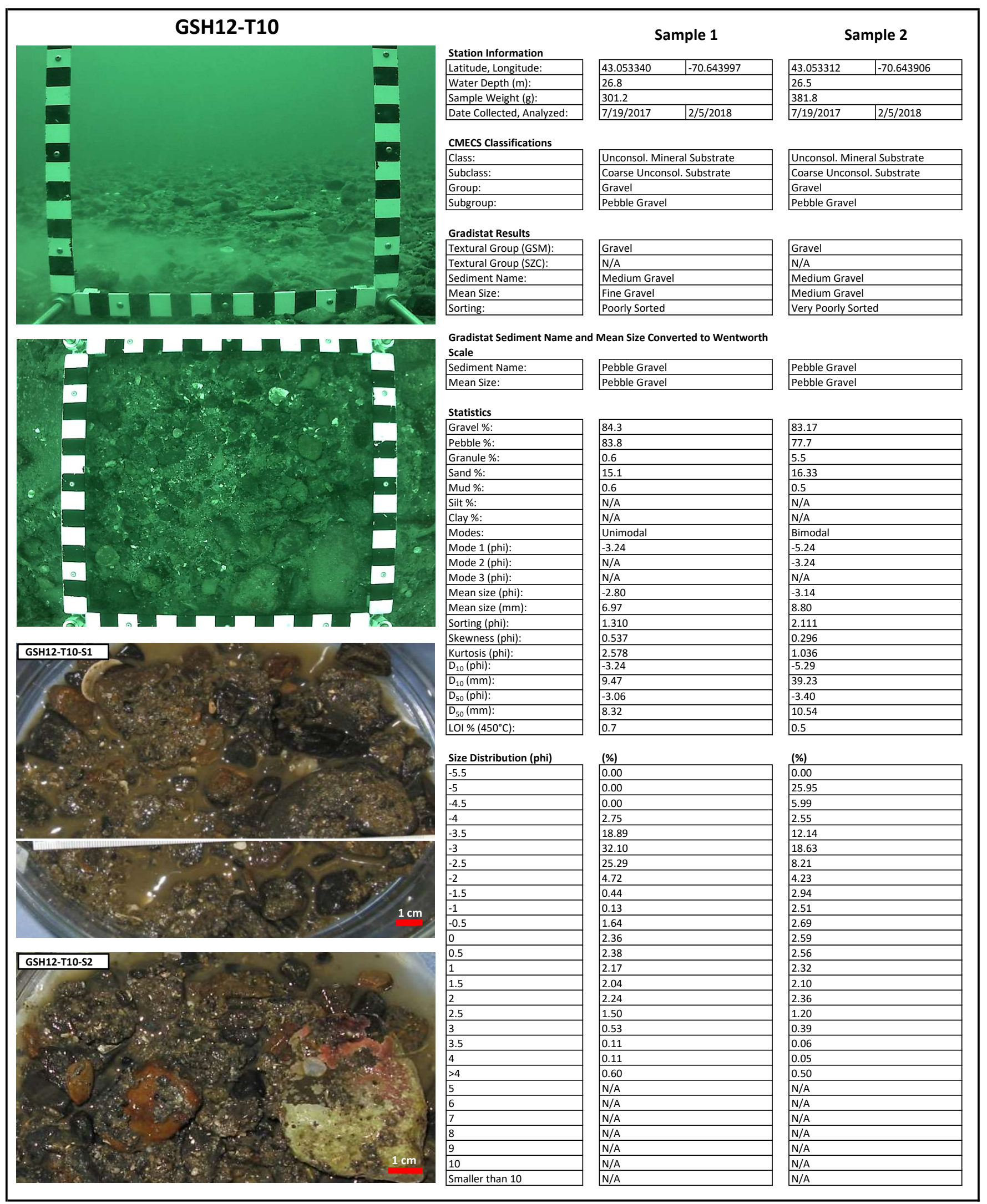




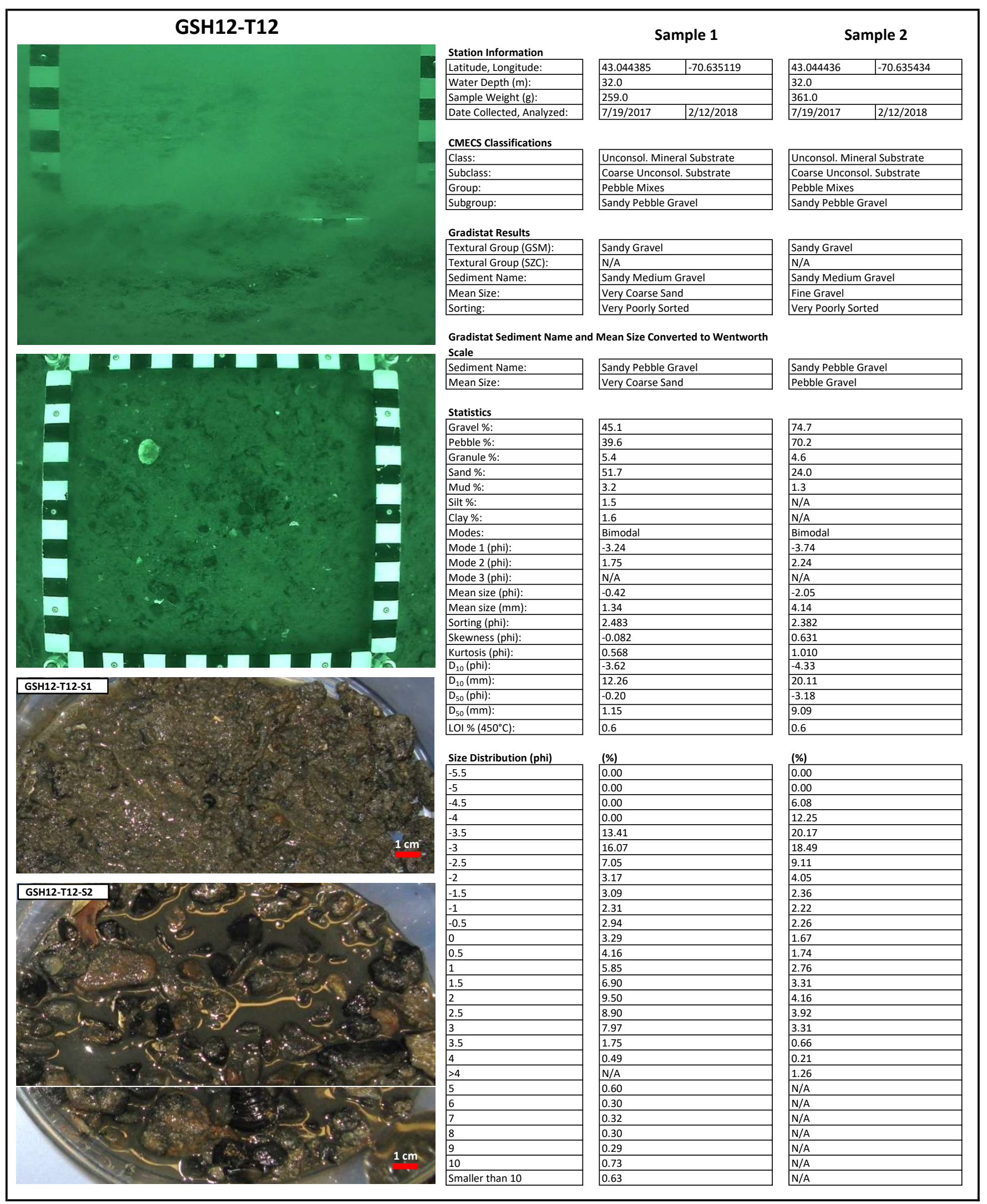




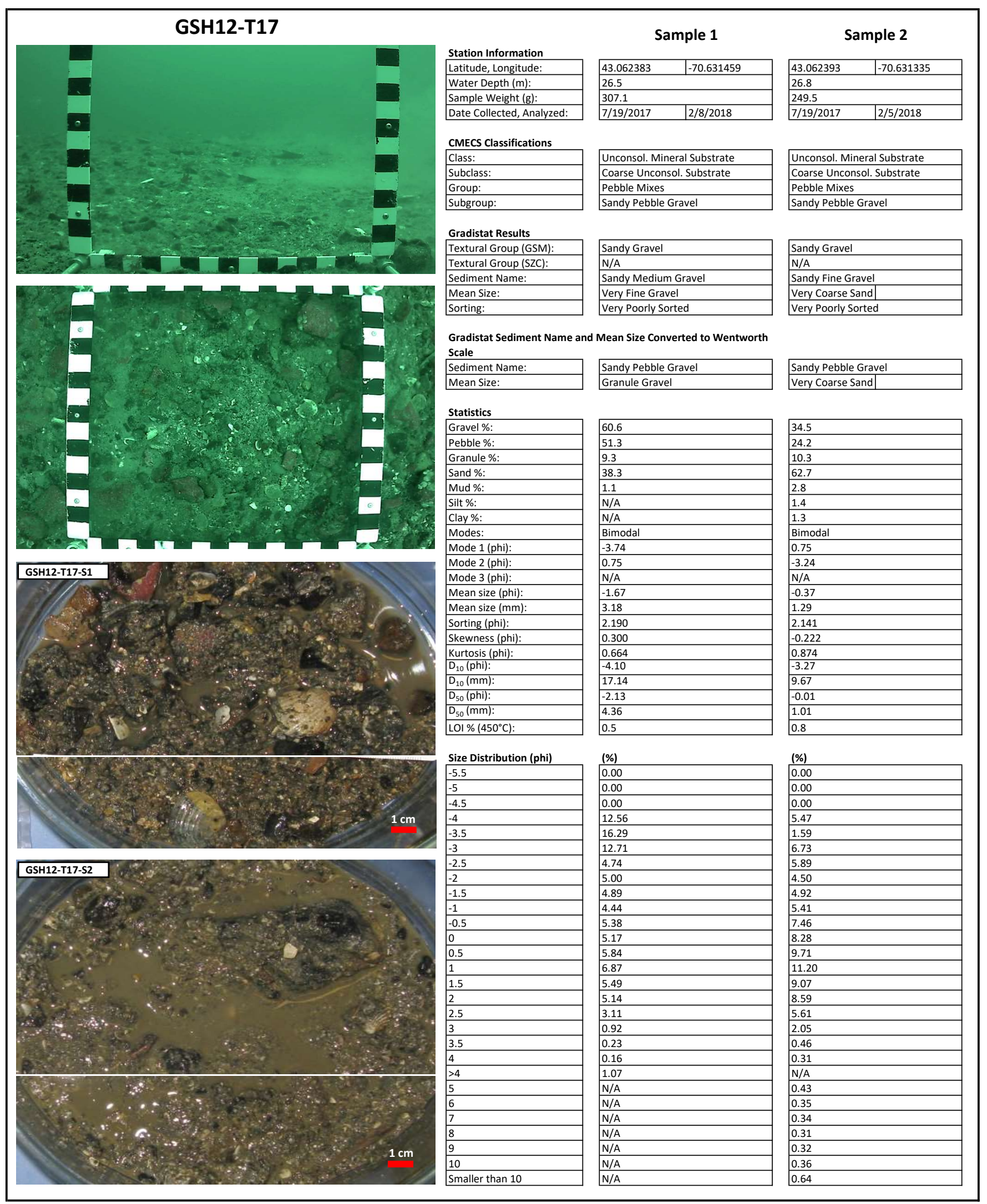



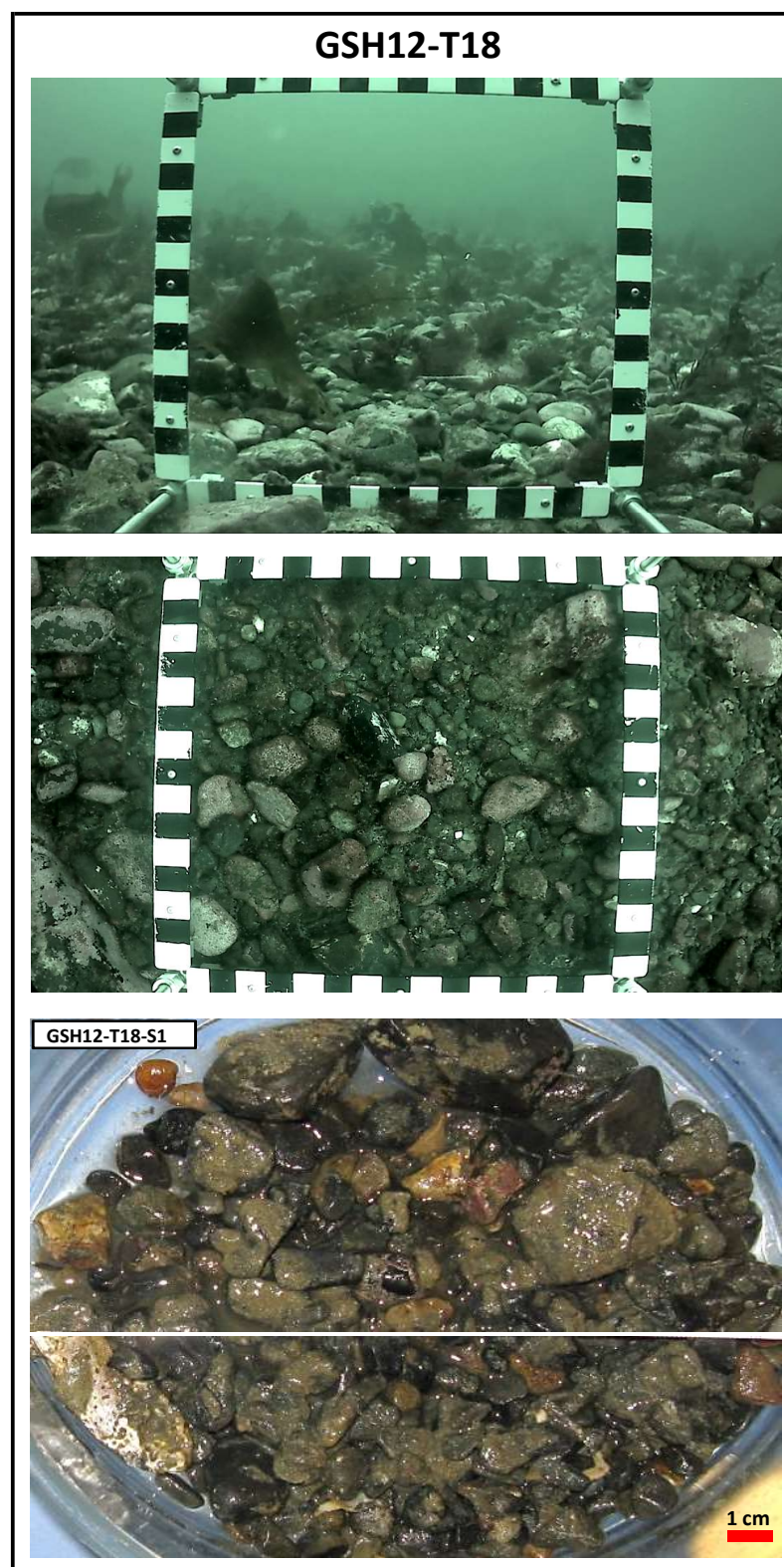

Station Information

Latitude, Longitude:

Water Depth (m):

Sample Weight (g):

Date Collected, Analyzed:

CMECS Classifications

Class:

Subclass:

Group:

Subgroup:

\section{Gradistat Results}

Textural Group (GSM):

Textural Group (SZC)

Sediment Name:

Mean Size:

Sorting:

Gradistat Sediment Name and Mean Size Converted to Wentworth

Scale

Sediment Name:

Mean Size:

Statistics

Gravel \%:

Pebble \%:

Granule \%:

Sand \%:

Mud \%:

\begin{tabular}{l} 
Silt \%: \\
\hline Clay \%: \\
\hline
\end{tabular}

Clay \%:

Mode 1 (phi):

\begin{tabular}{l} 
Mode 1 (phi): \\
\hline Mode 2 (phi):
\end{tabular}

Mode 3 (phi):

Mean size (phi):

Mean size $(\mathrm{mm})$ :

Sorting (phi):

Skewness (phi):

Kurtosis (phi):

$\mathrm{D}_{10}$ (phi):

$\mathrm{D}_{10}(\mathrm{~mm})$ :

$\mathrm{D}_{50}$ (phi):

$\mathrm{D}_{50}(\mathrm{~mm}):$

LOI \% $\left(450^{\circ} \mathrm{C}\right)$ :

Size Distribution (phi)
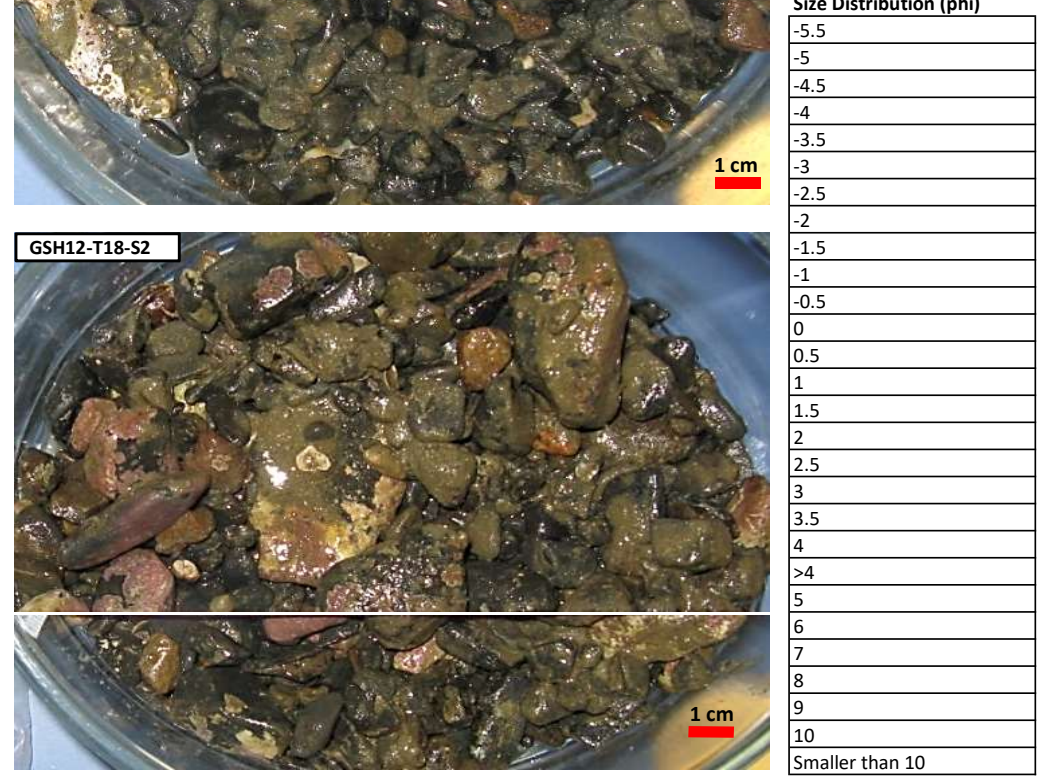

\begin{tabular}{ll}
\hline-2.5 \\
\hline-2 \\
\hline-1.5 \\
\hline-12
\end{tabular}

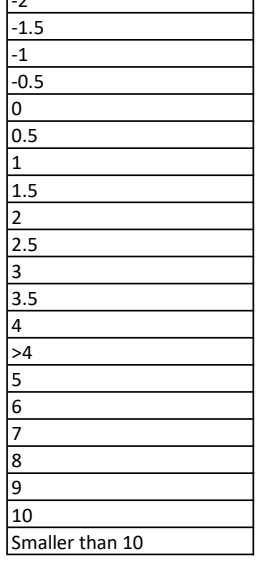

Sample 1

\begin{tabular}{|l|l|}
\hline 43.078743 & -70.645144 \\
\hline 15.2 \\
\hline 336.7 & $2 / 5 / 2018$ \\
\hline $8 / 1 / 2017$ & \\
\hline
\end{tabular}

Unconsol. Mineral Substrate Coarse Unconsol. Substrate

\section{Gravel}

Pebble Gravel

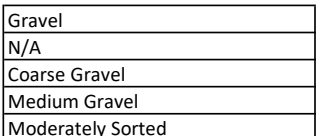

Moderately Sorted

Sample 2

\begin{tabular}{|l|l|}
\hline 43.078727 & -70.645136 \\
\hline 15.2 & \\
\hline 598.9 & $2 / 5 / 2018$ \\
\hline $8 / 1 / 2017$ & \\
\hline
\end{tabular}

\section{Unconsol. Mineral Substrate} Coarse Unconsol. Substrate

Gravel

\begin{tabular}{|l}
\hline Pebble Gravel \\
\hline
\end{tabular}

\begin{tabular}{|l|}
\hline Gravel \\
\hline N/A \\
\hline Coarse Gravel \\
\hline Medium Gravel \\
\hline Moderately Sorted \\
\hline
\end{tabular}

\begin{tabular}{|l|}
\hline Pebble Gravel \\
\hline Pebble Gravel \\
\hline
\end{tabular}

\begin{tabular}{|l|}
\hline Pebble Gravel \\
\hline Pebble Gravel \\
\hline
\end{tabular}
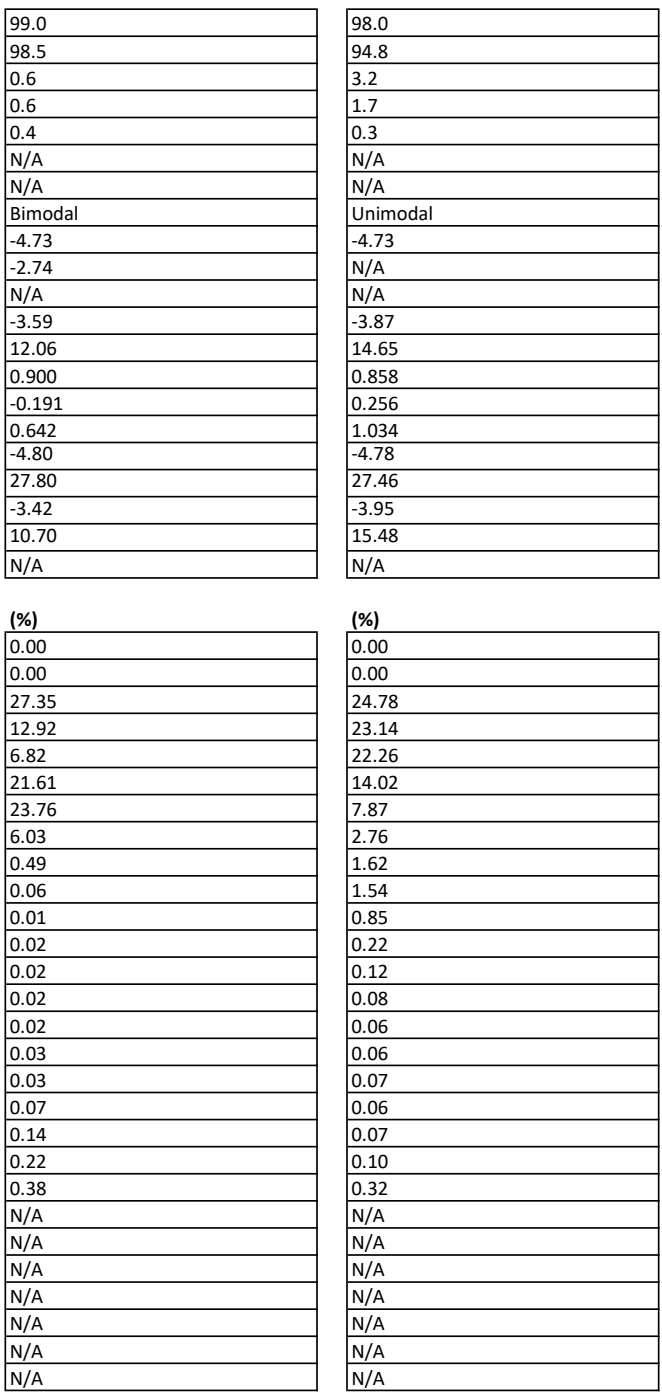


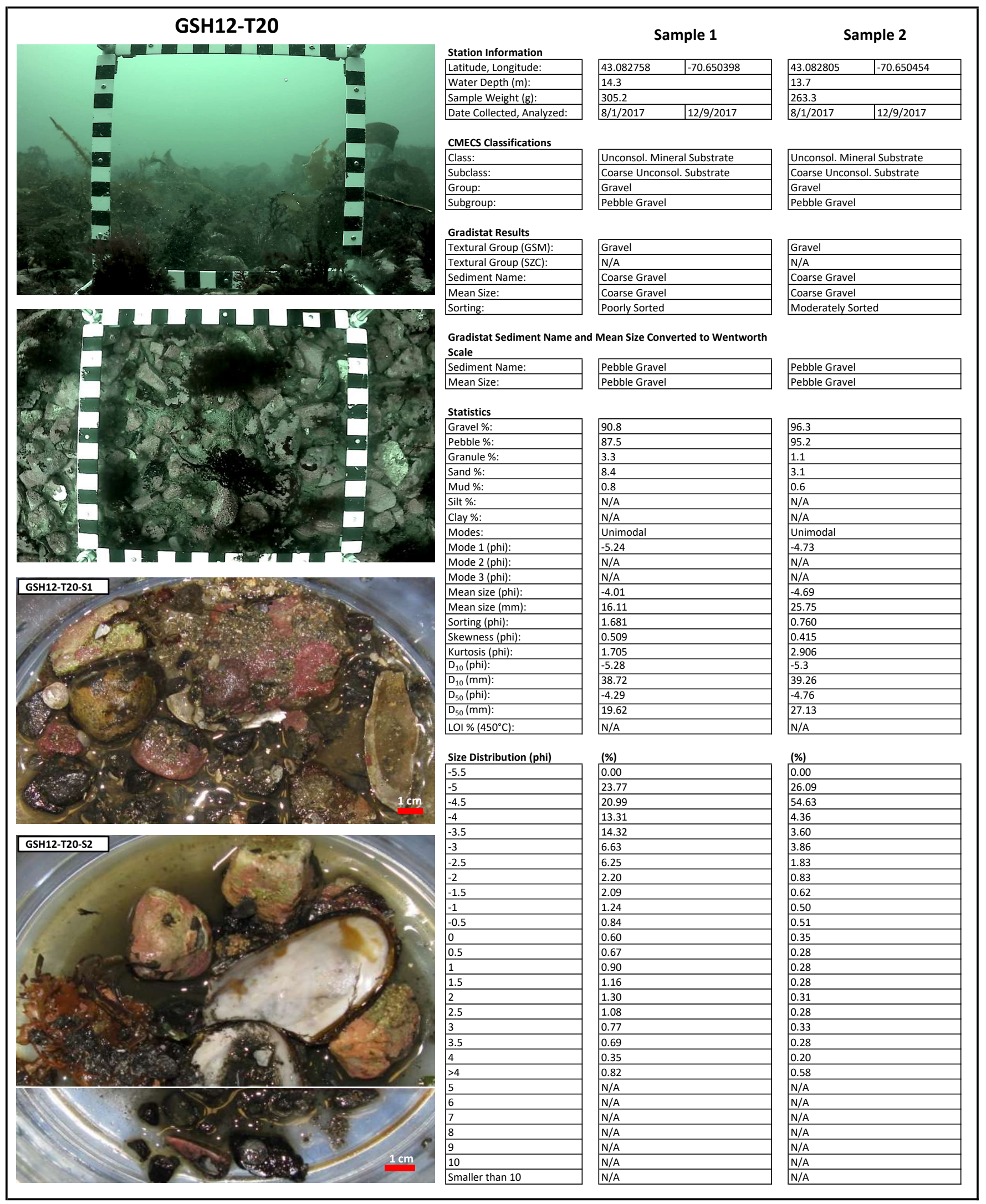




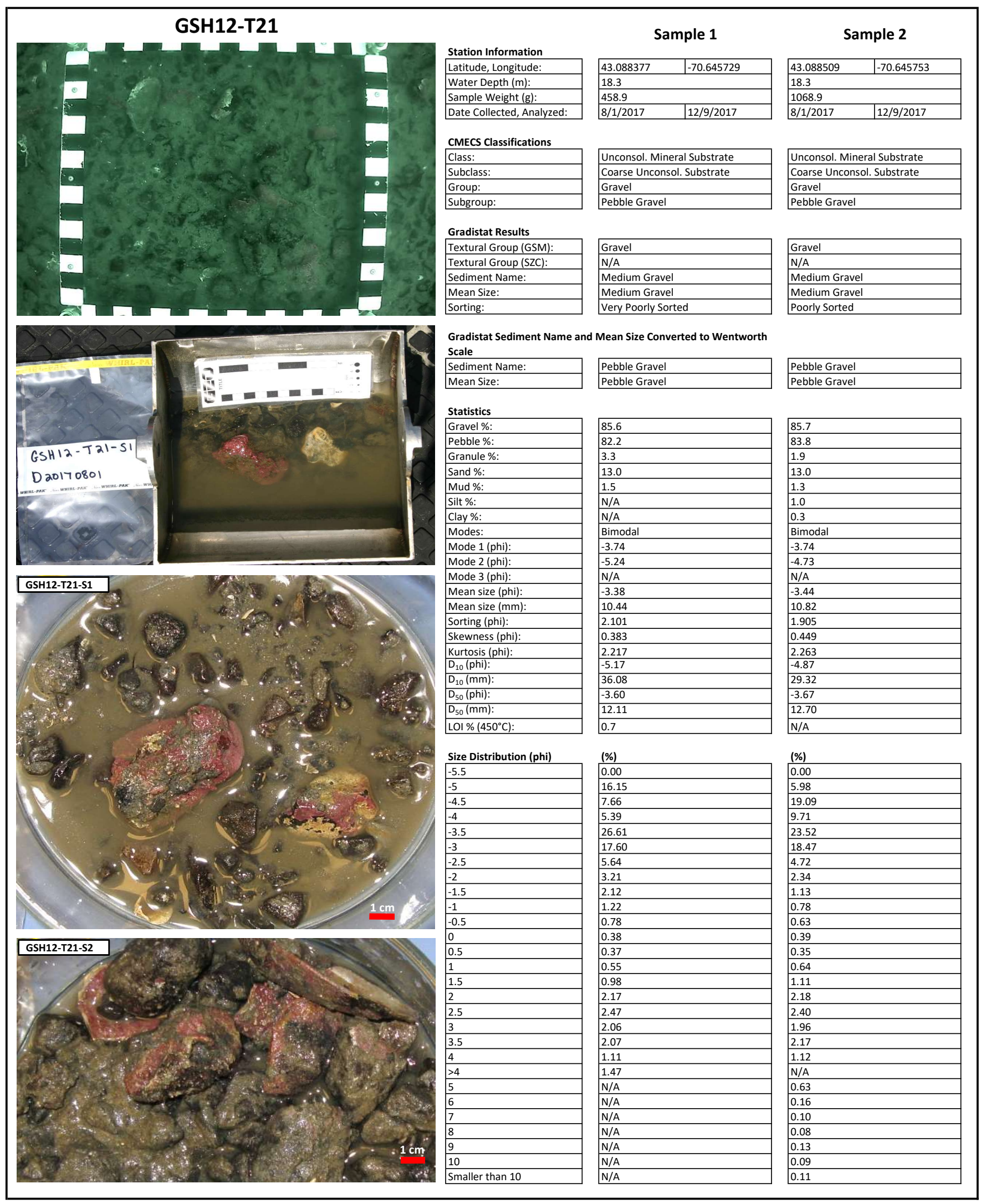




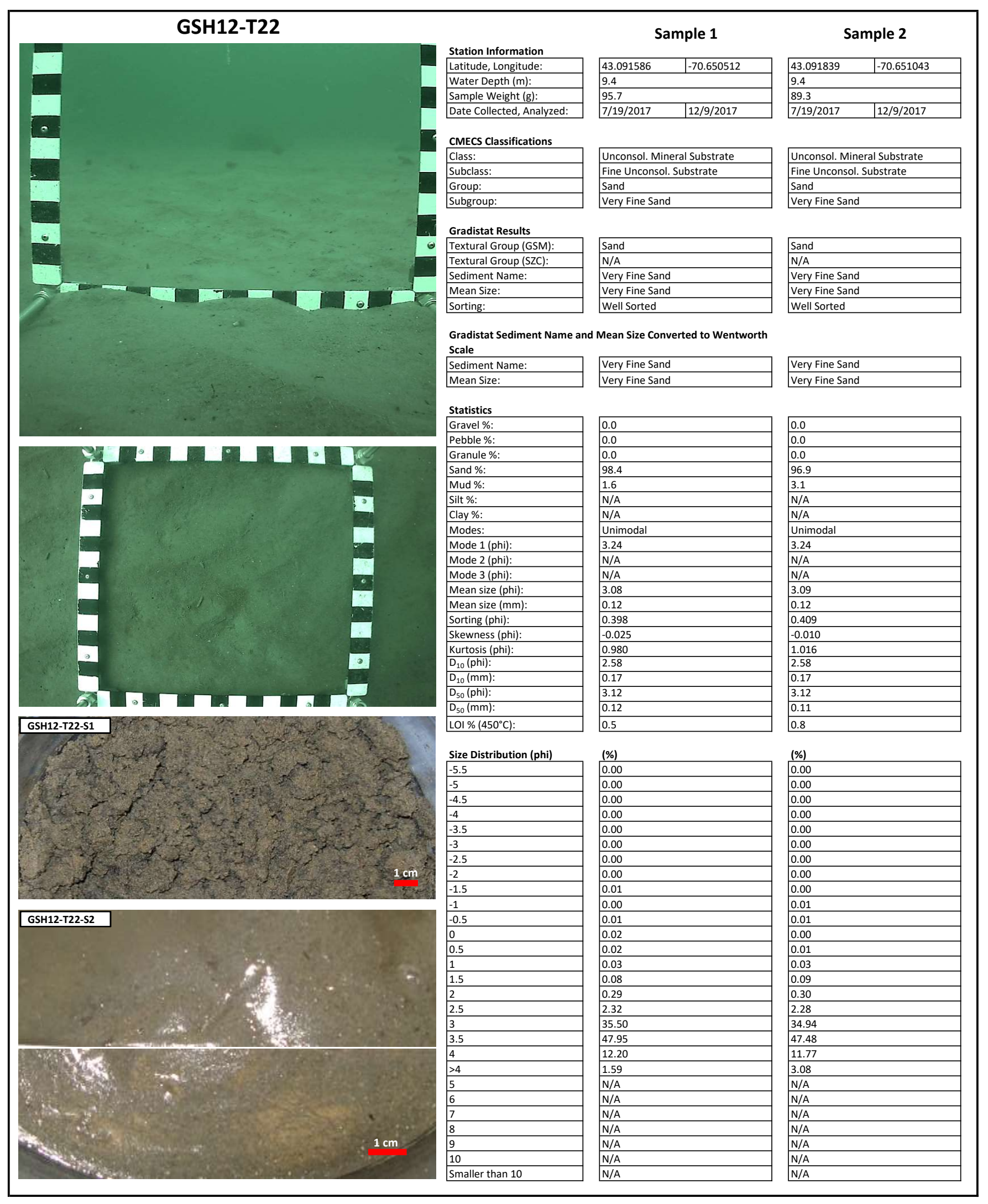




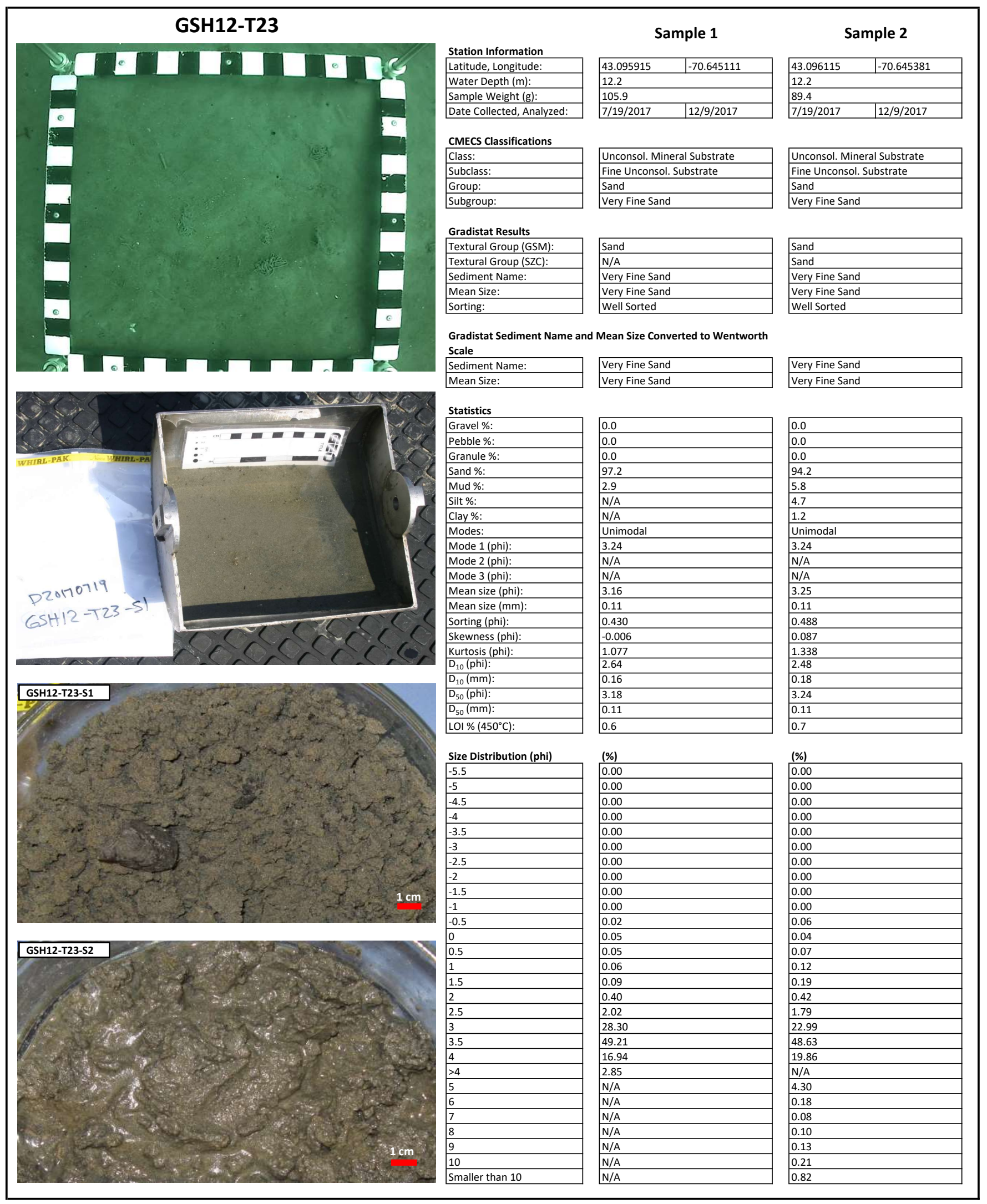




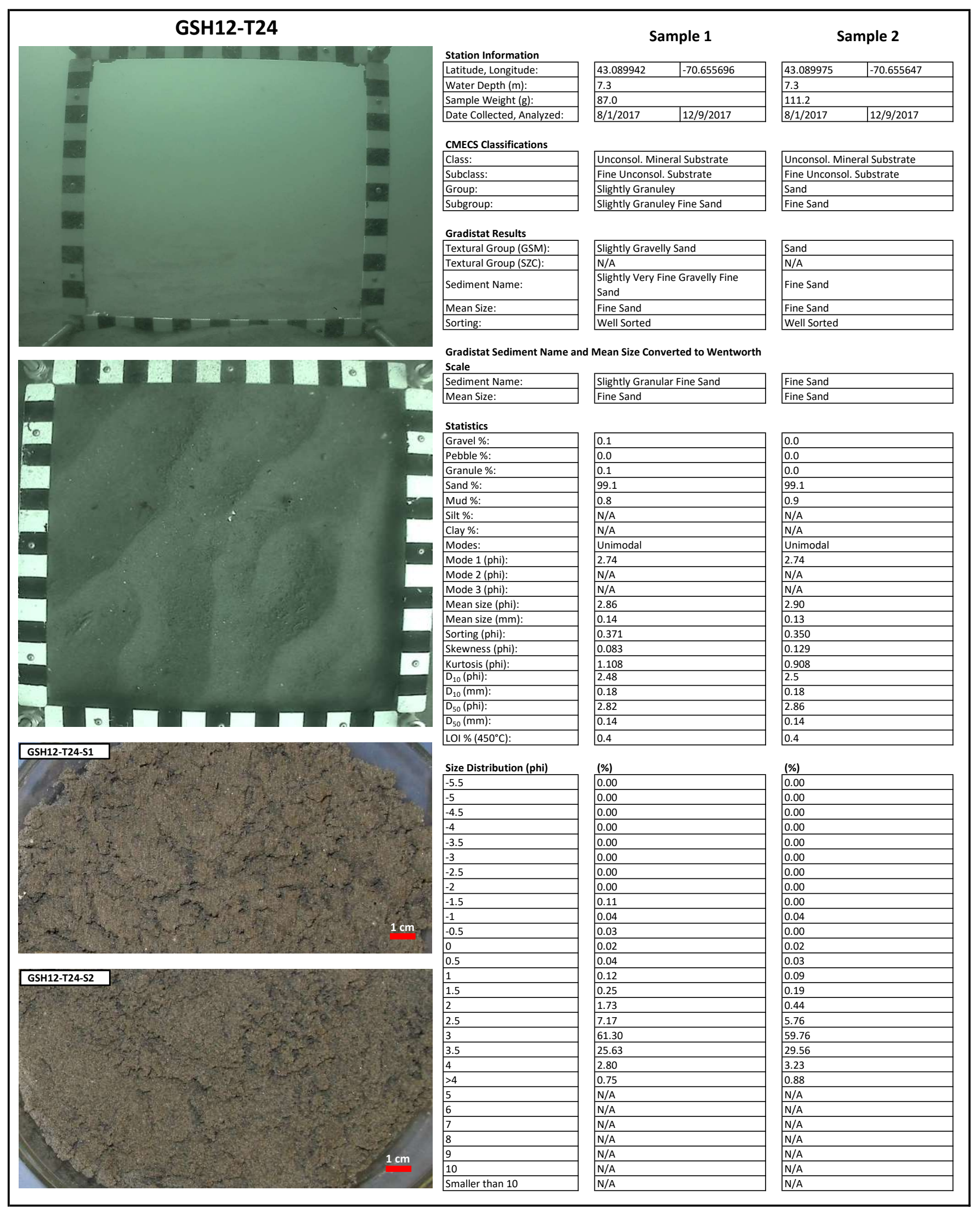




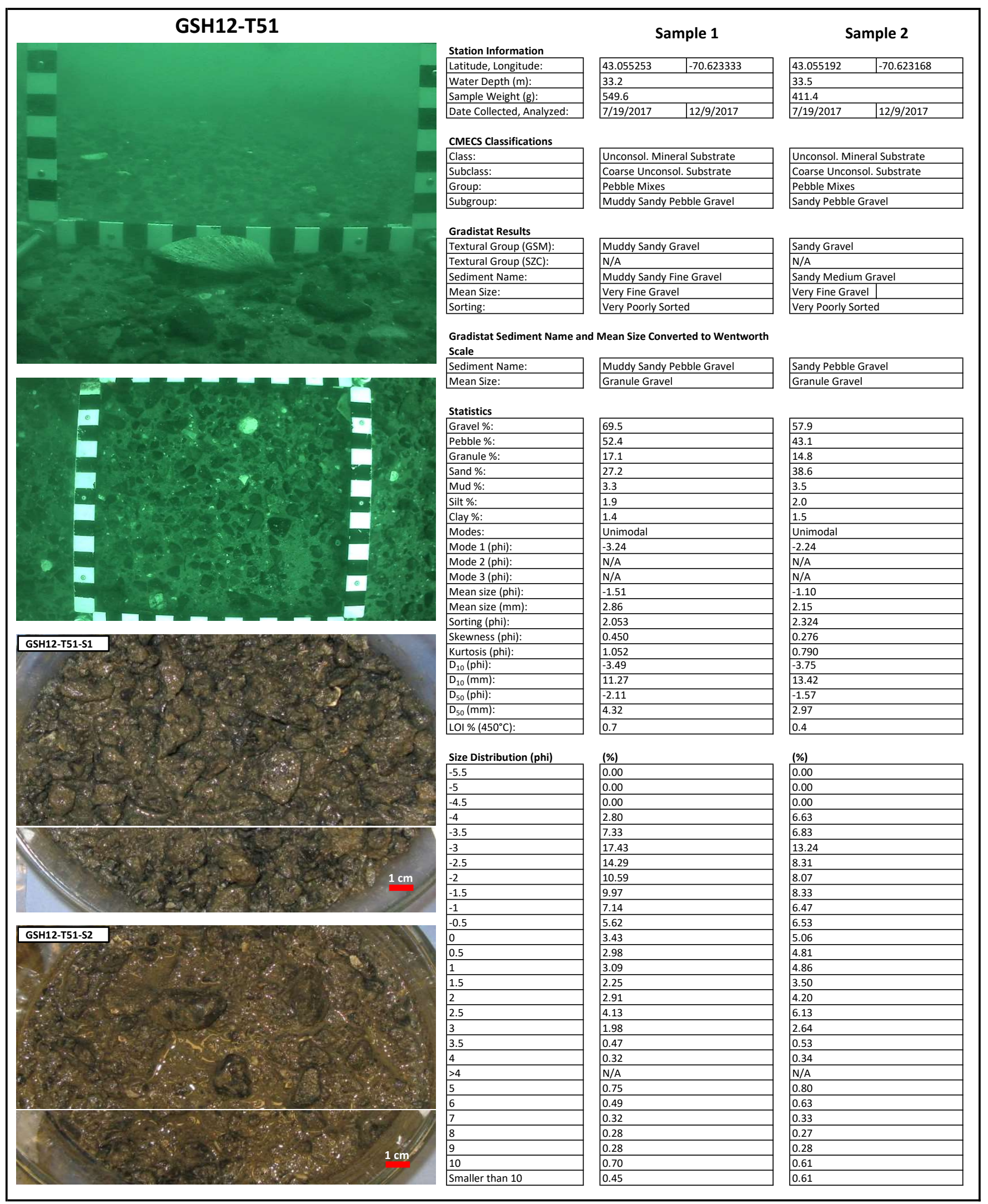




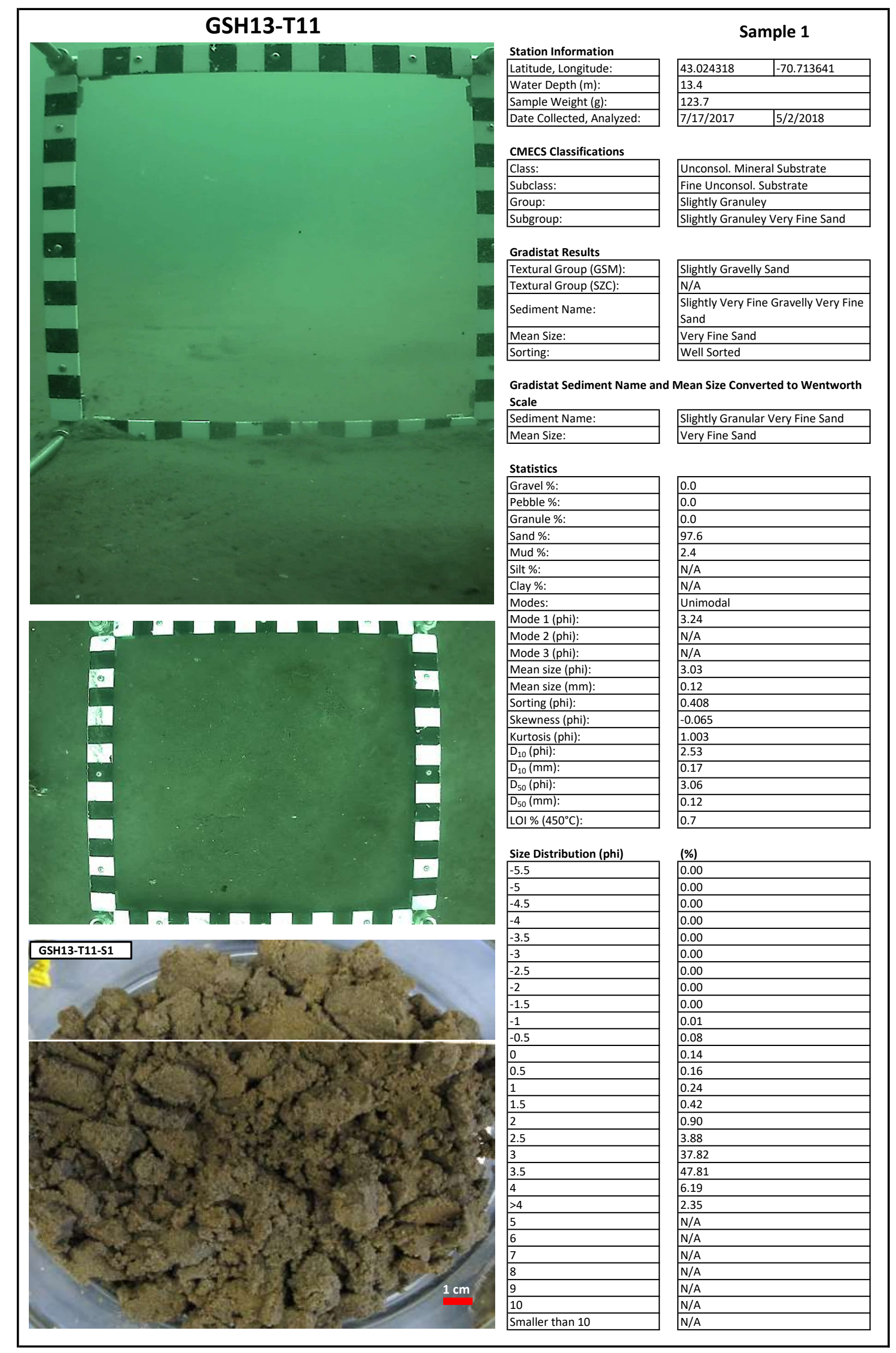




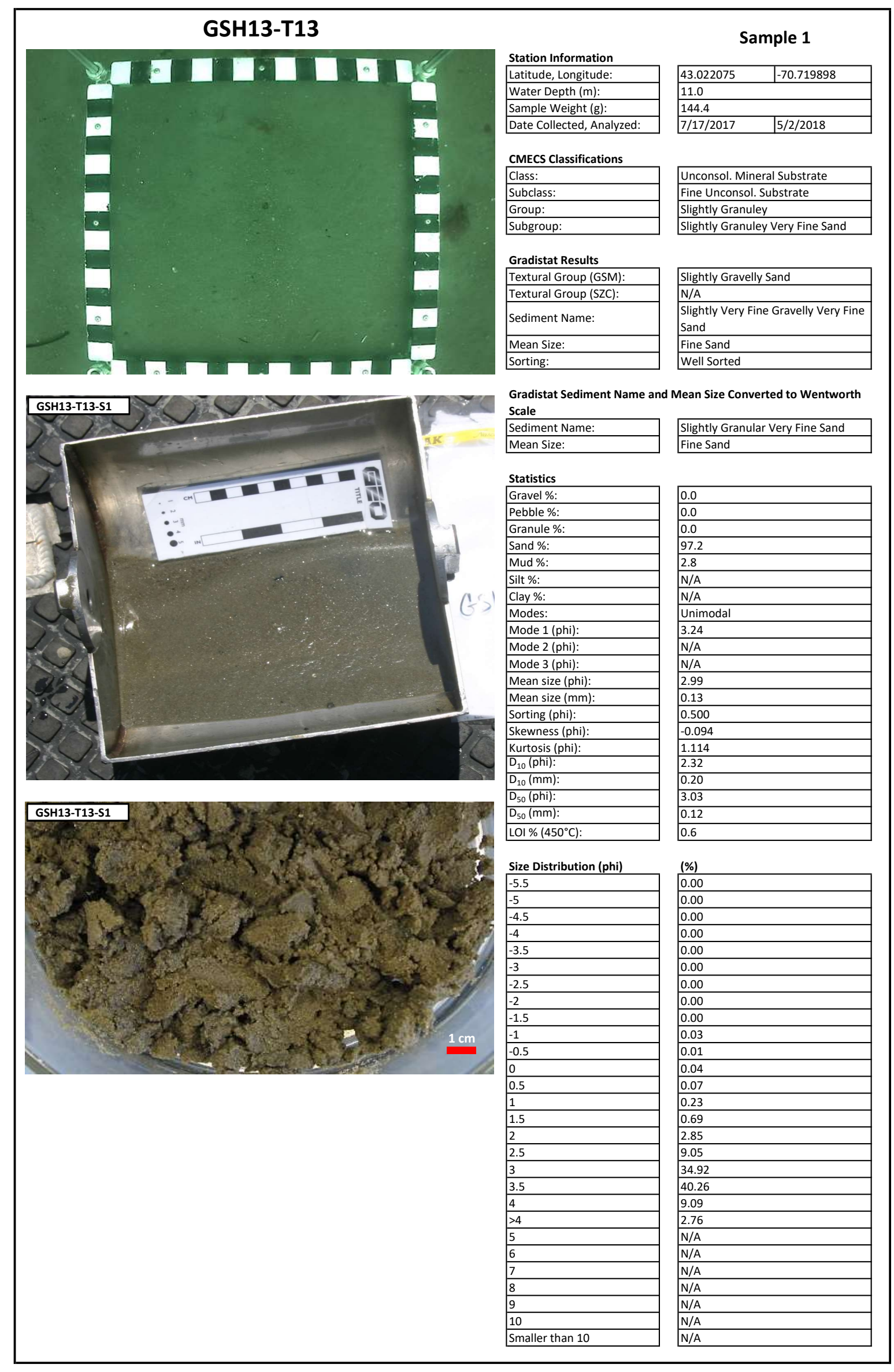




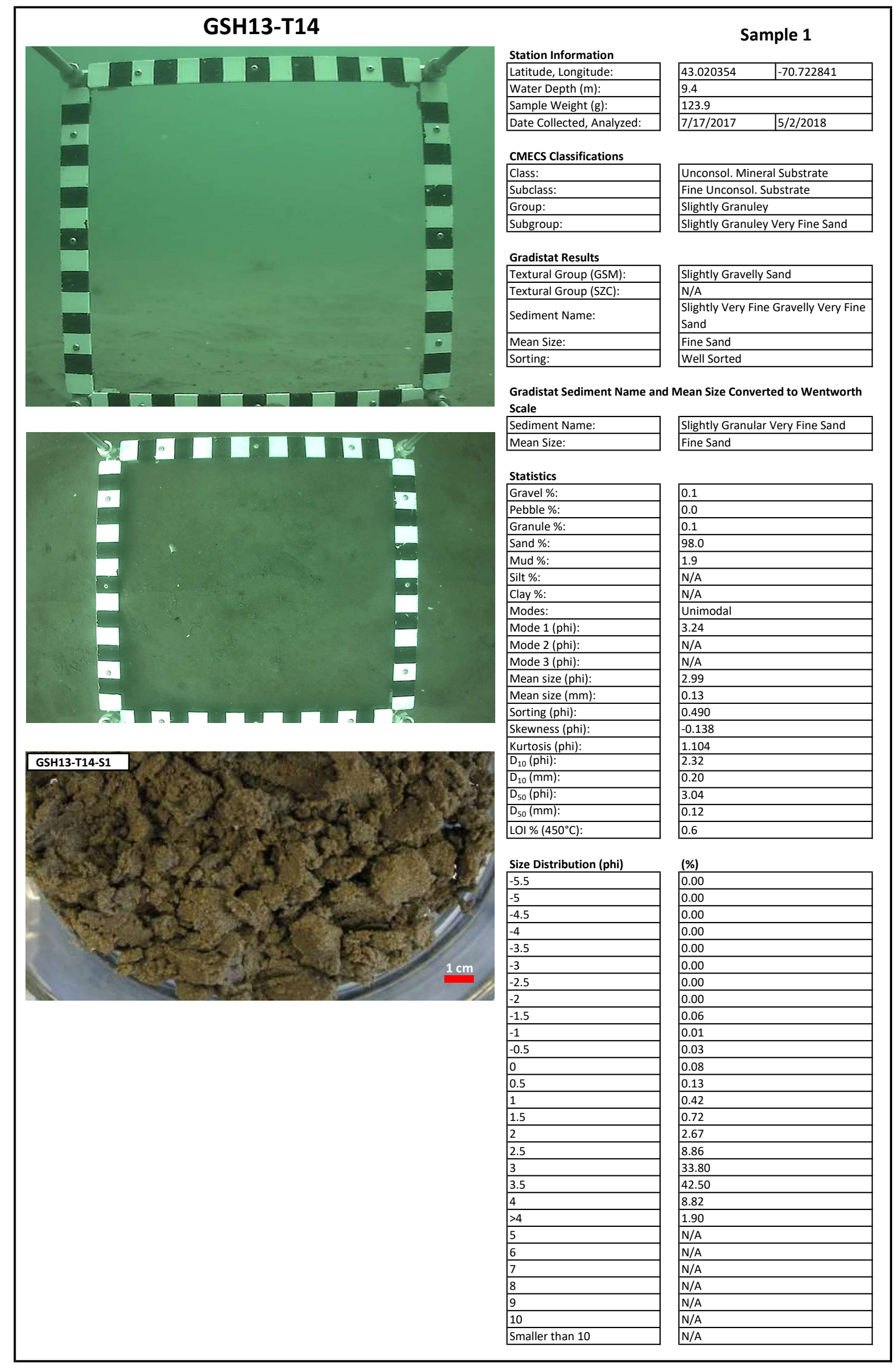




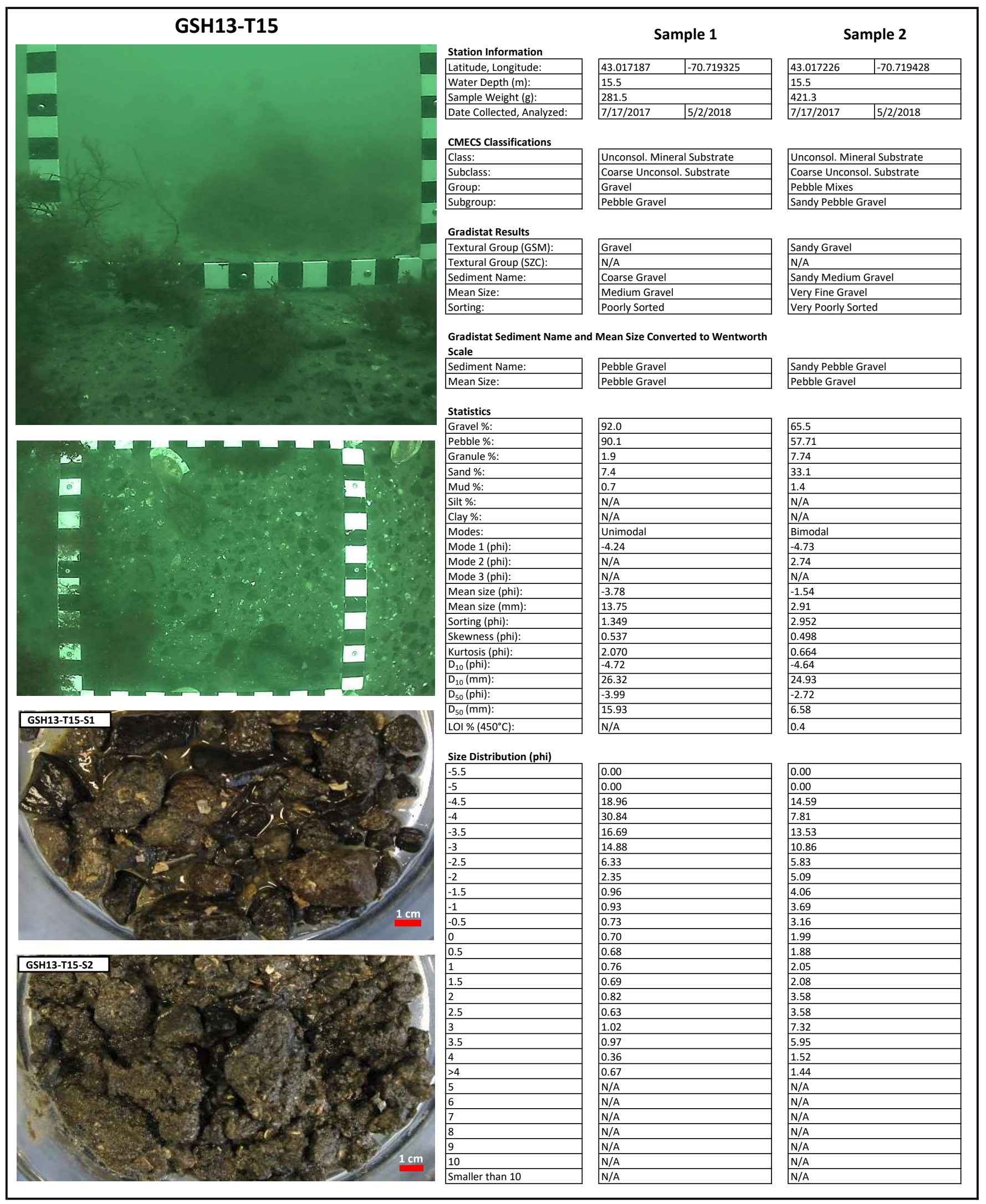




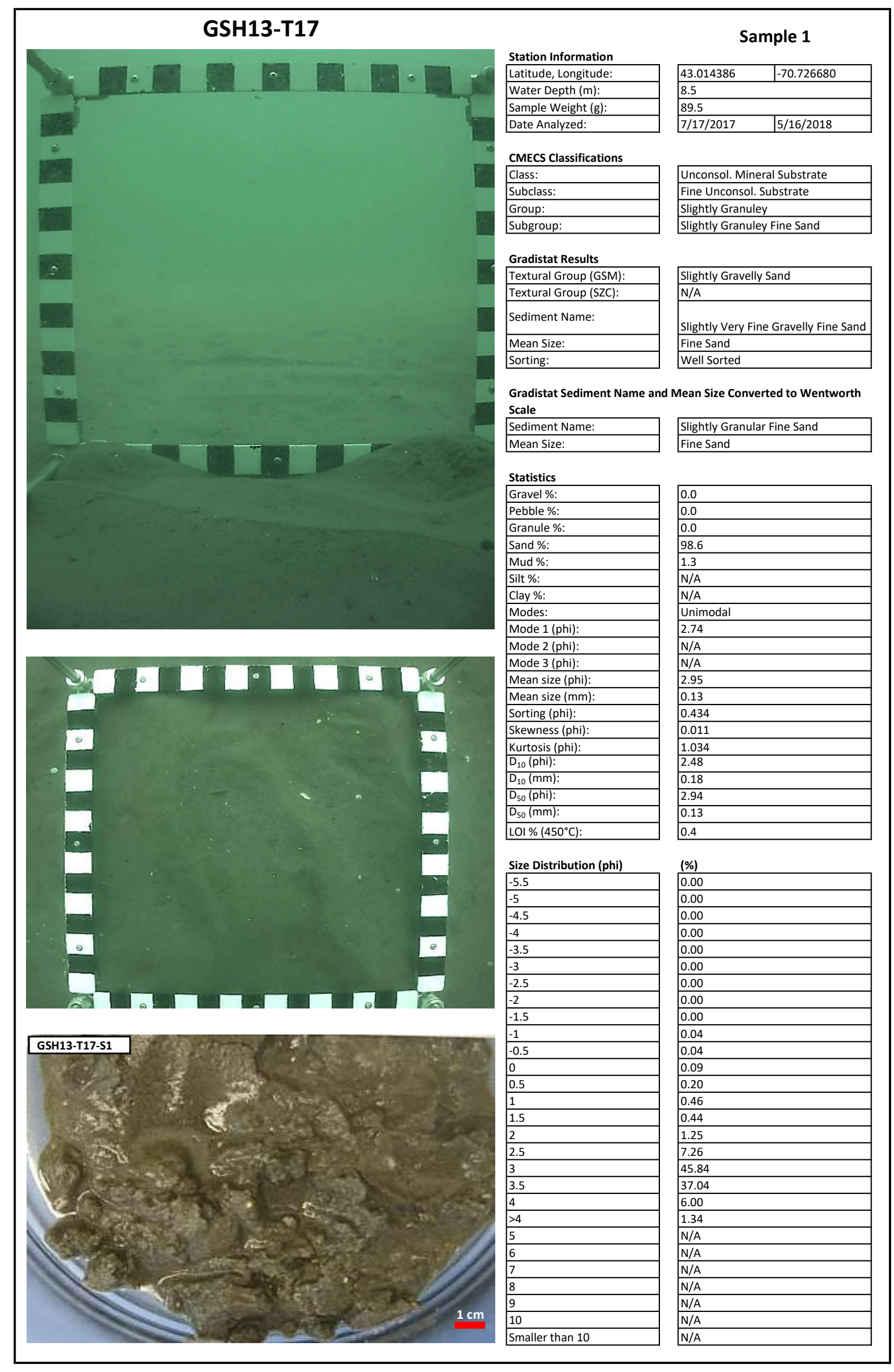




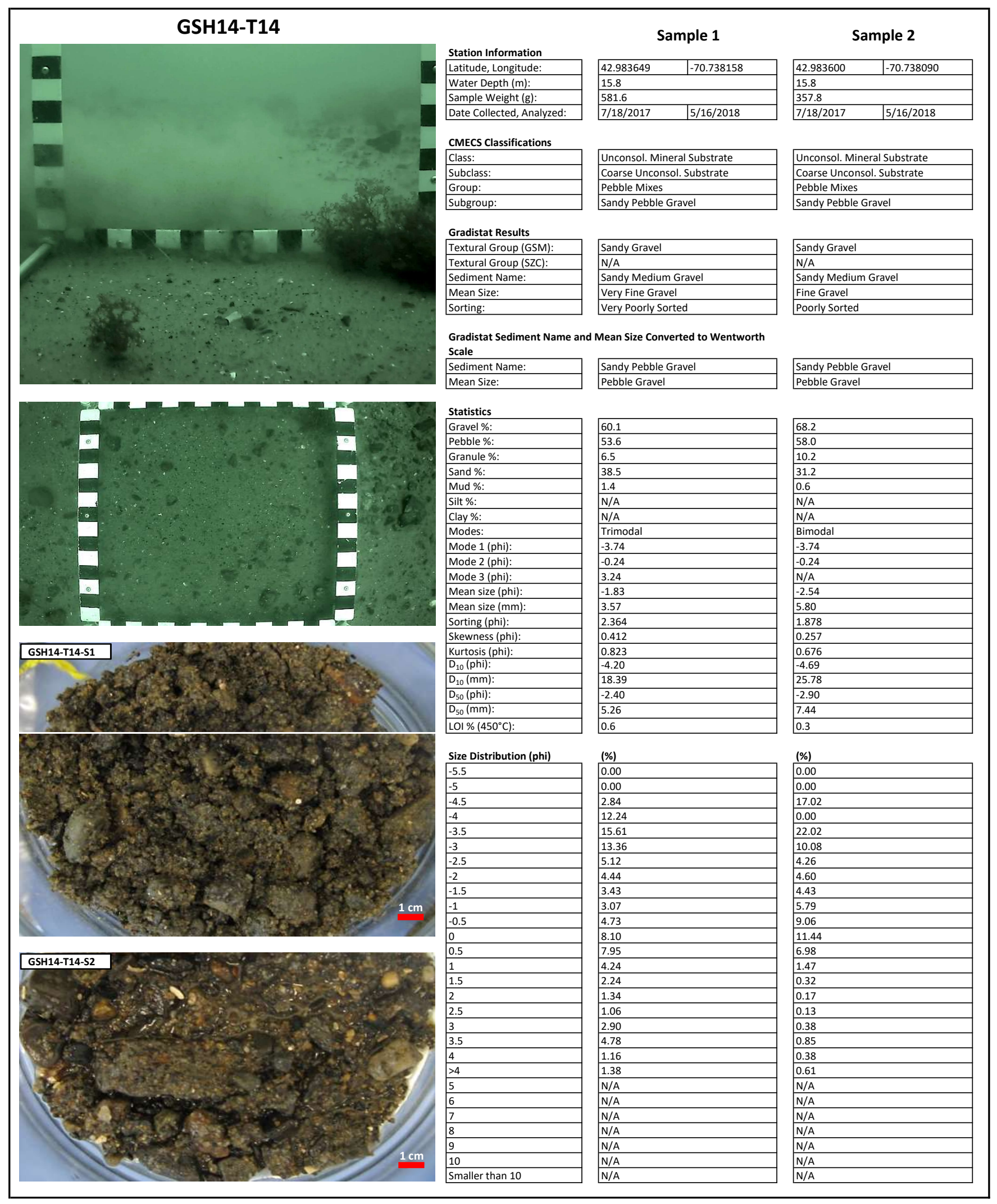




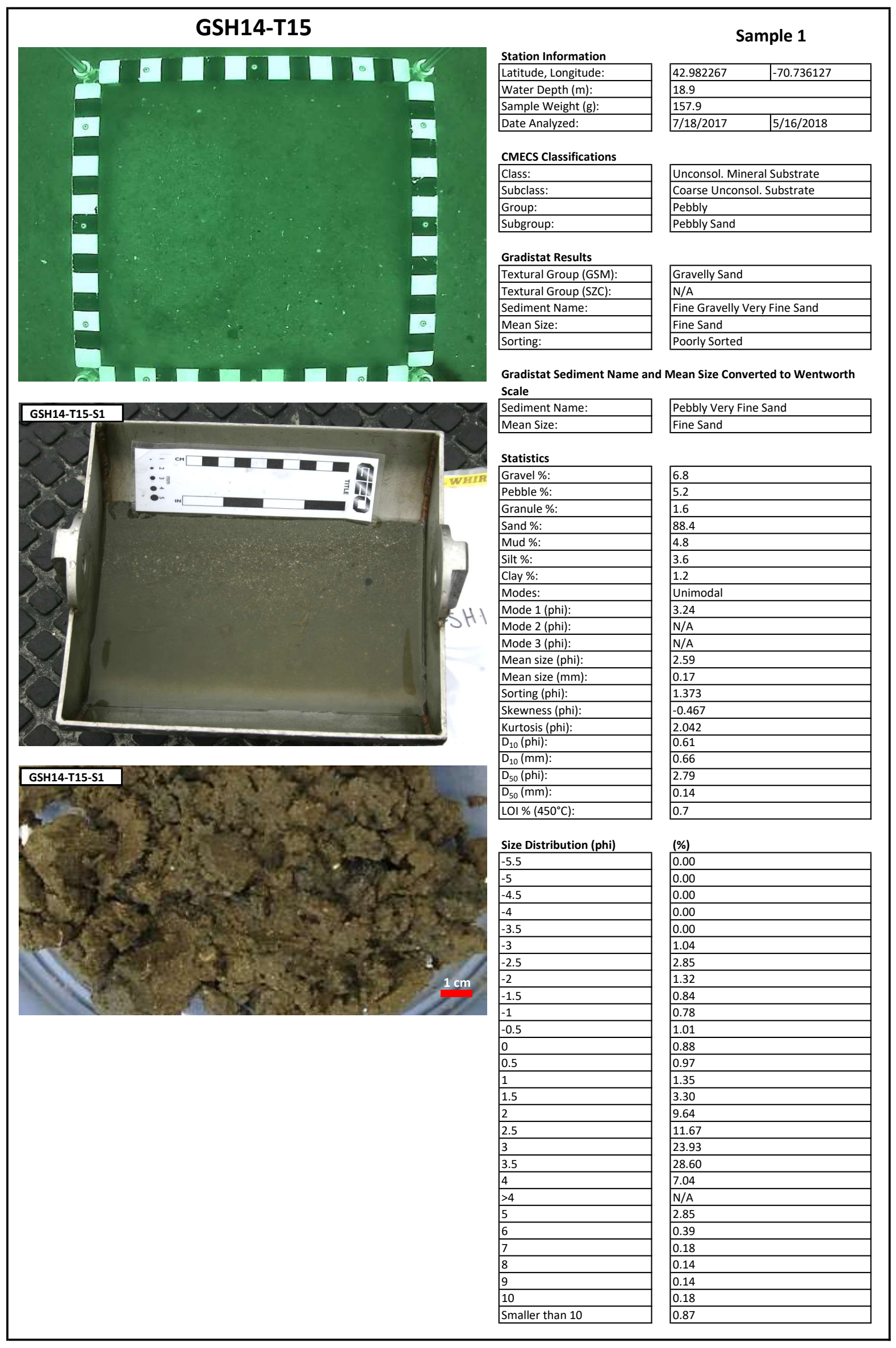




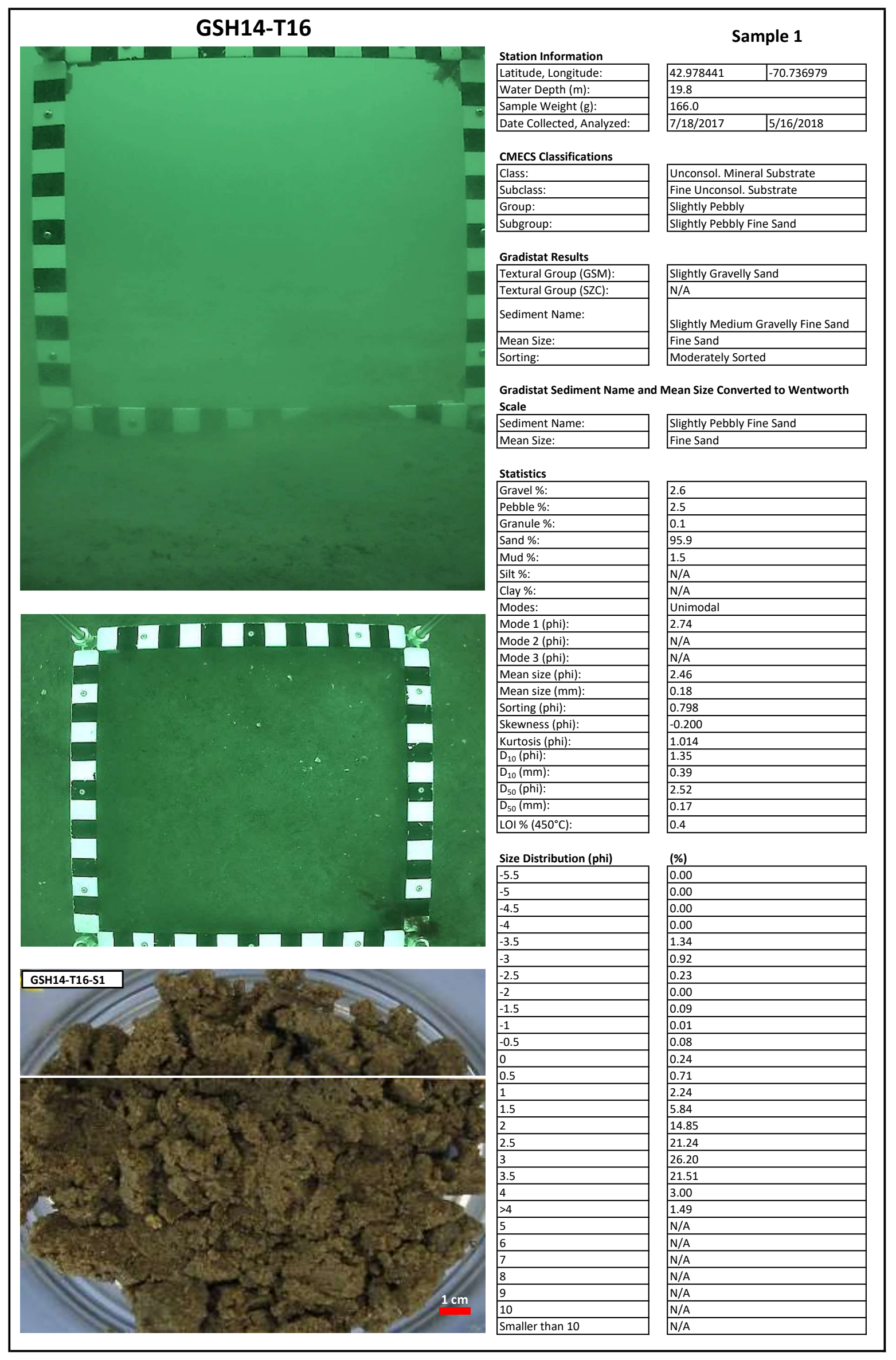




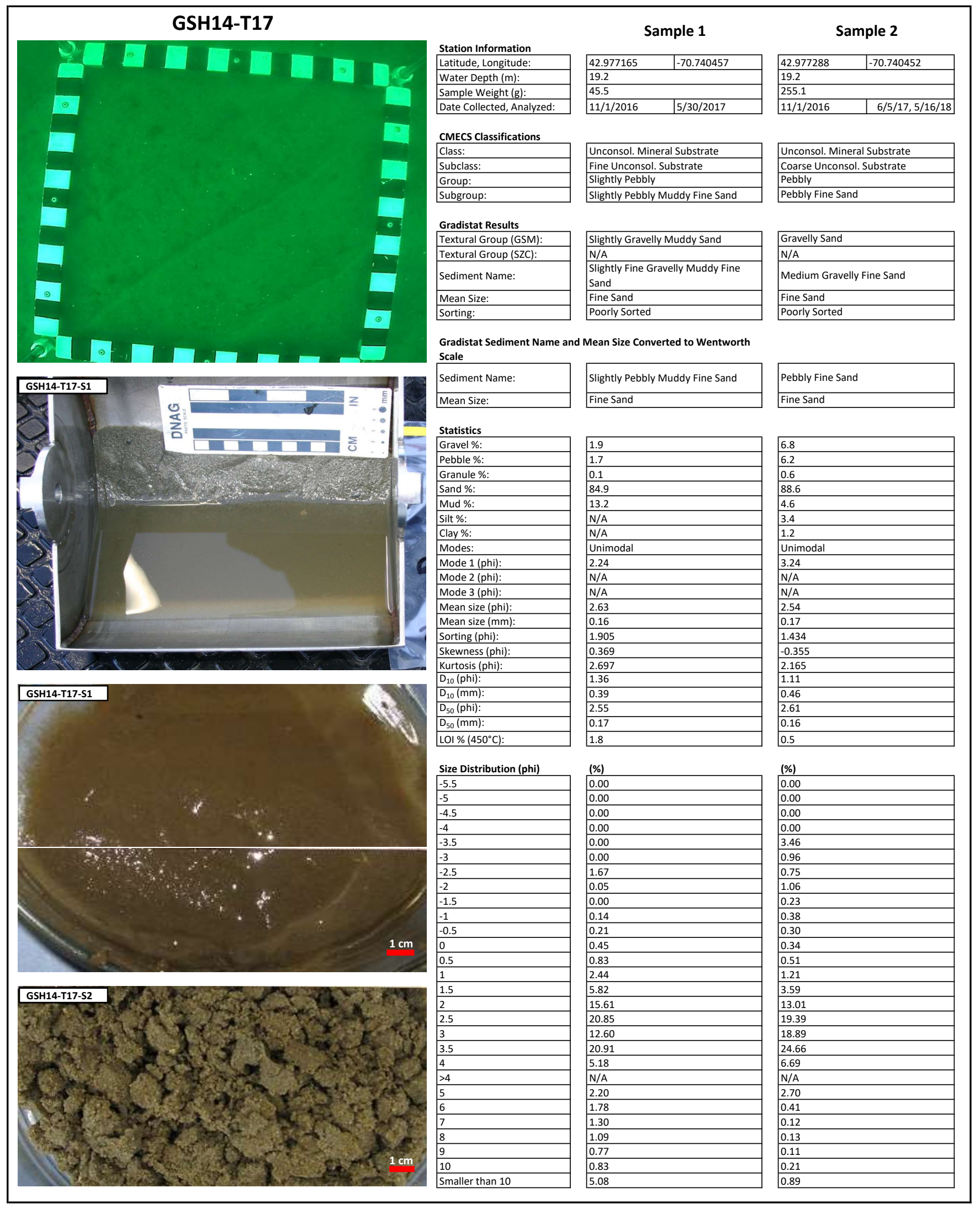




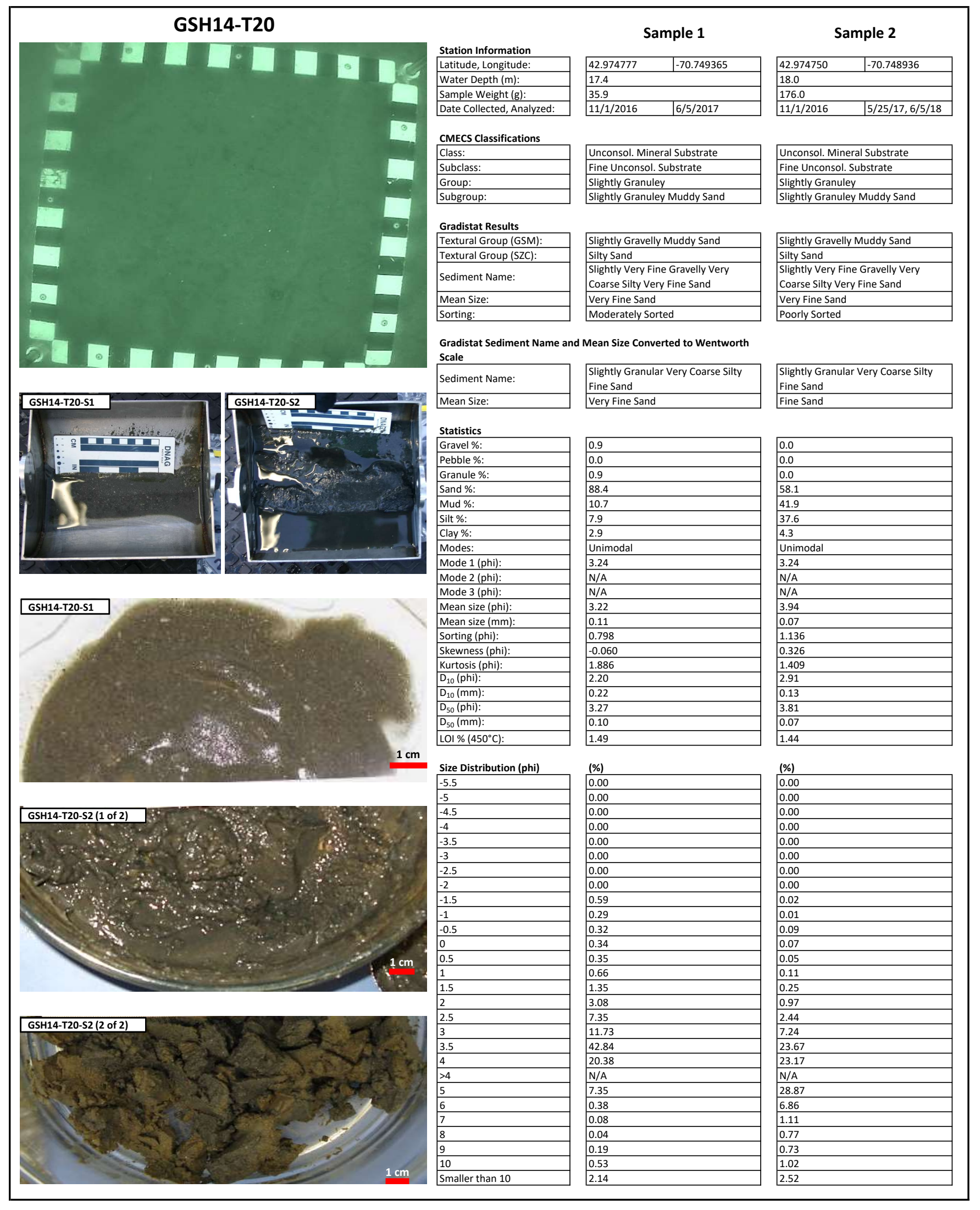




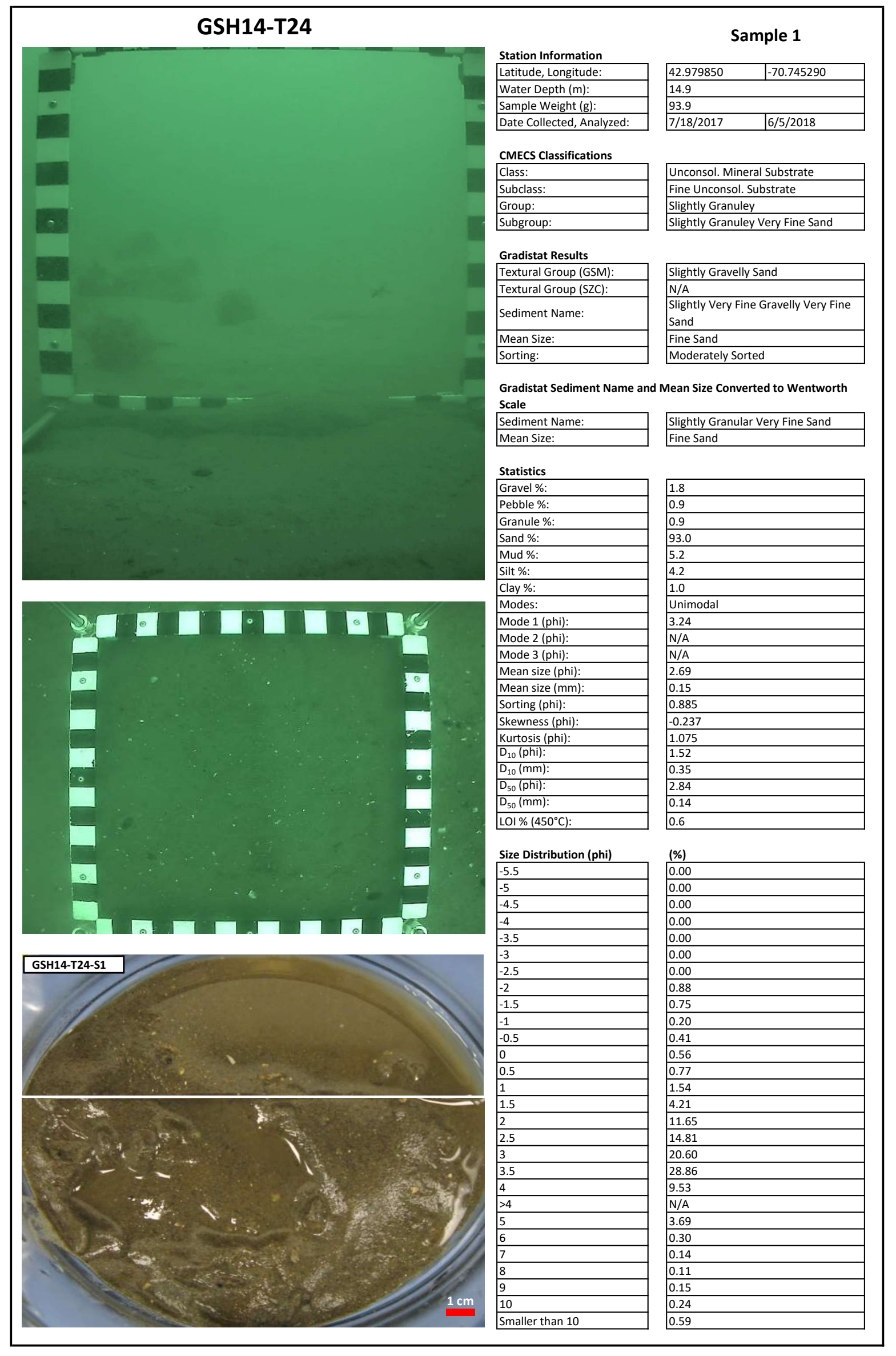




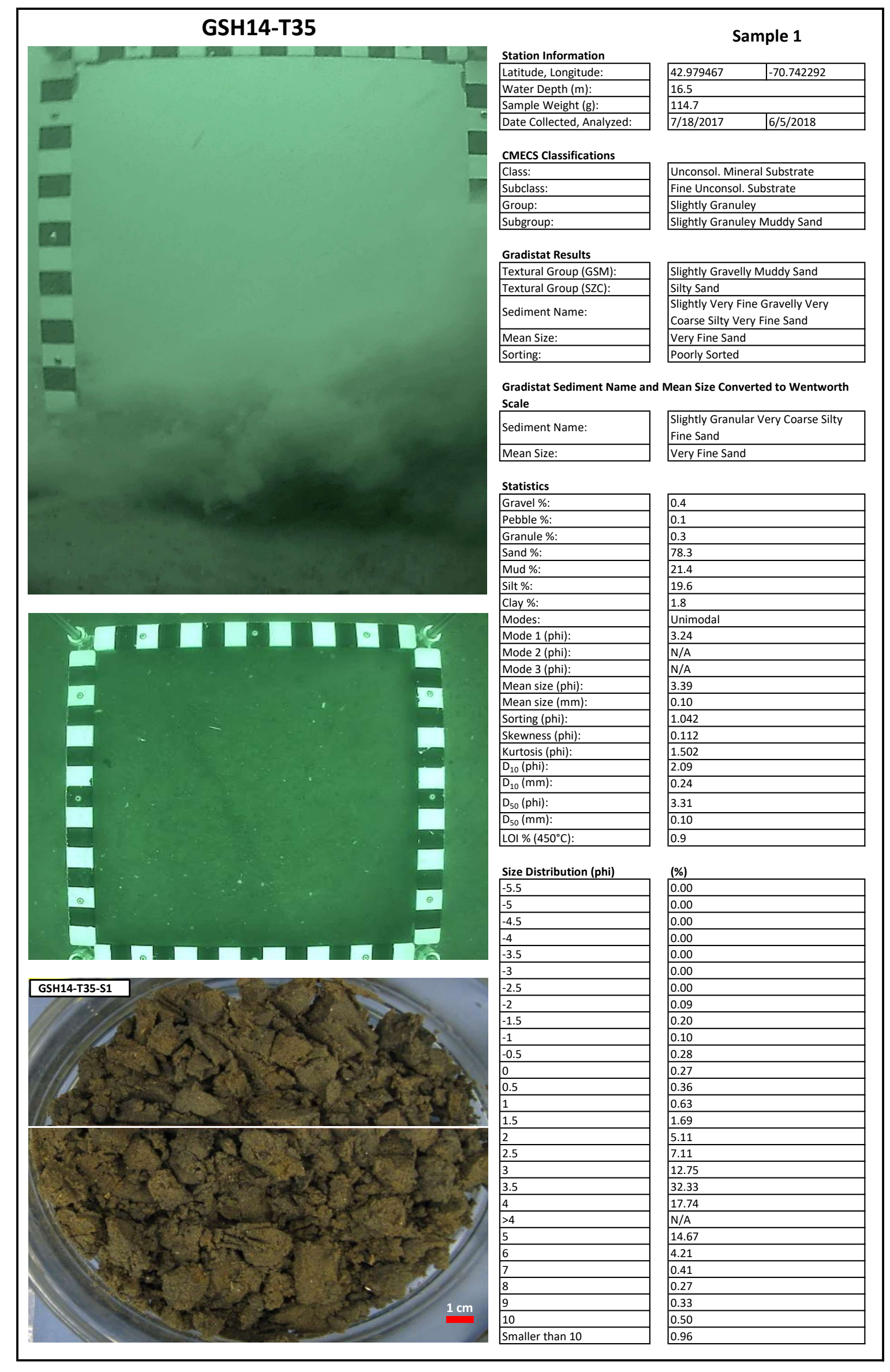




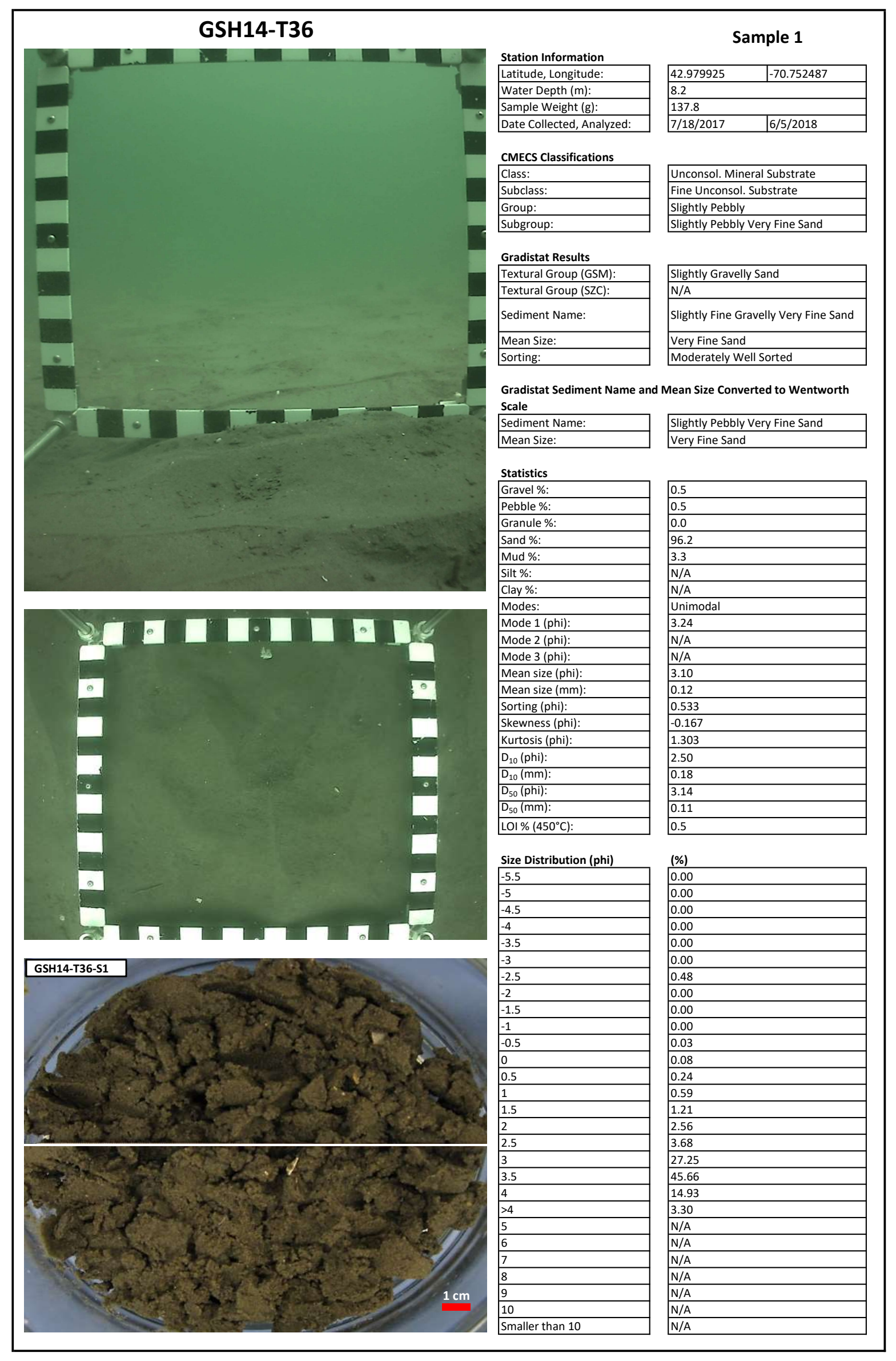




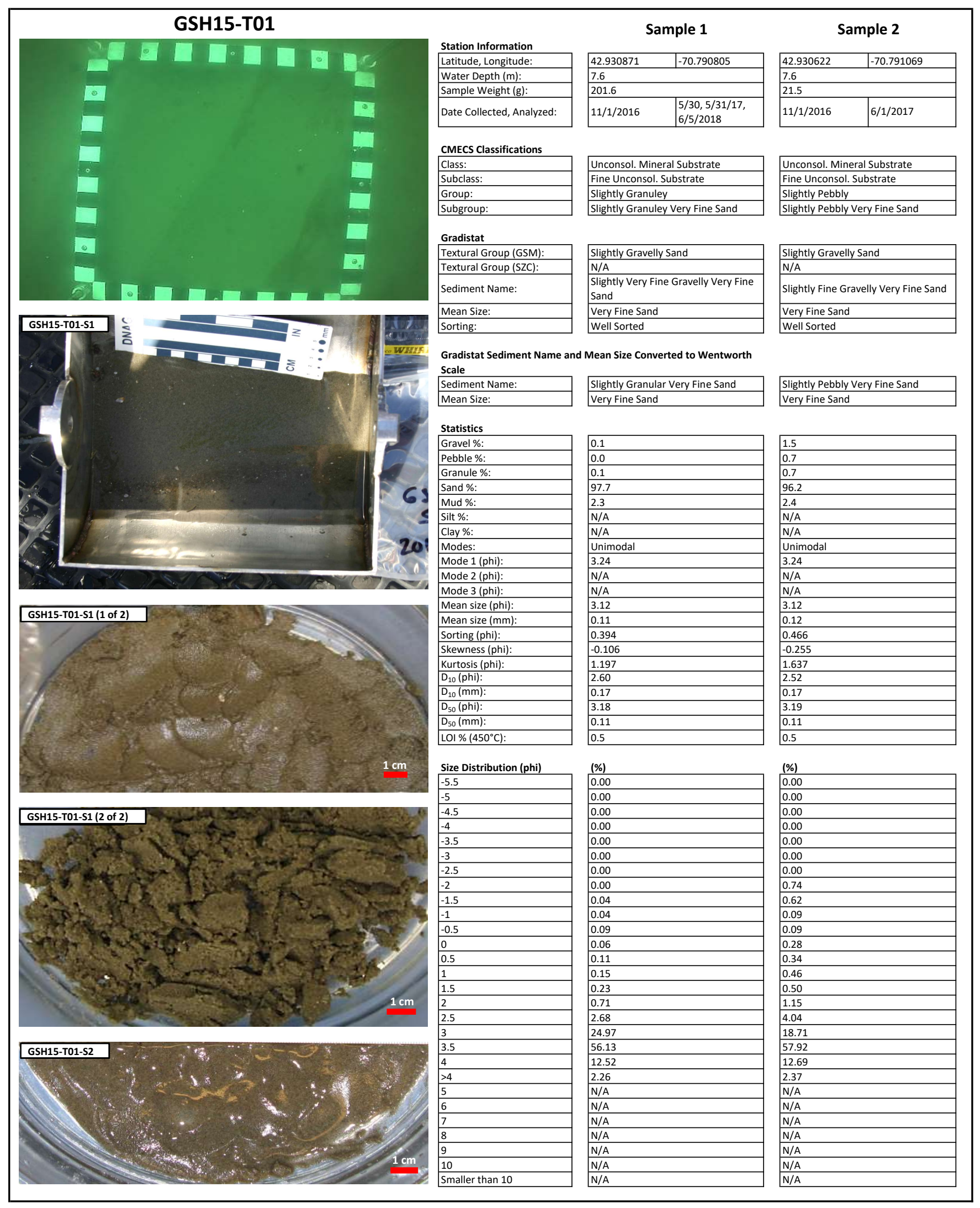




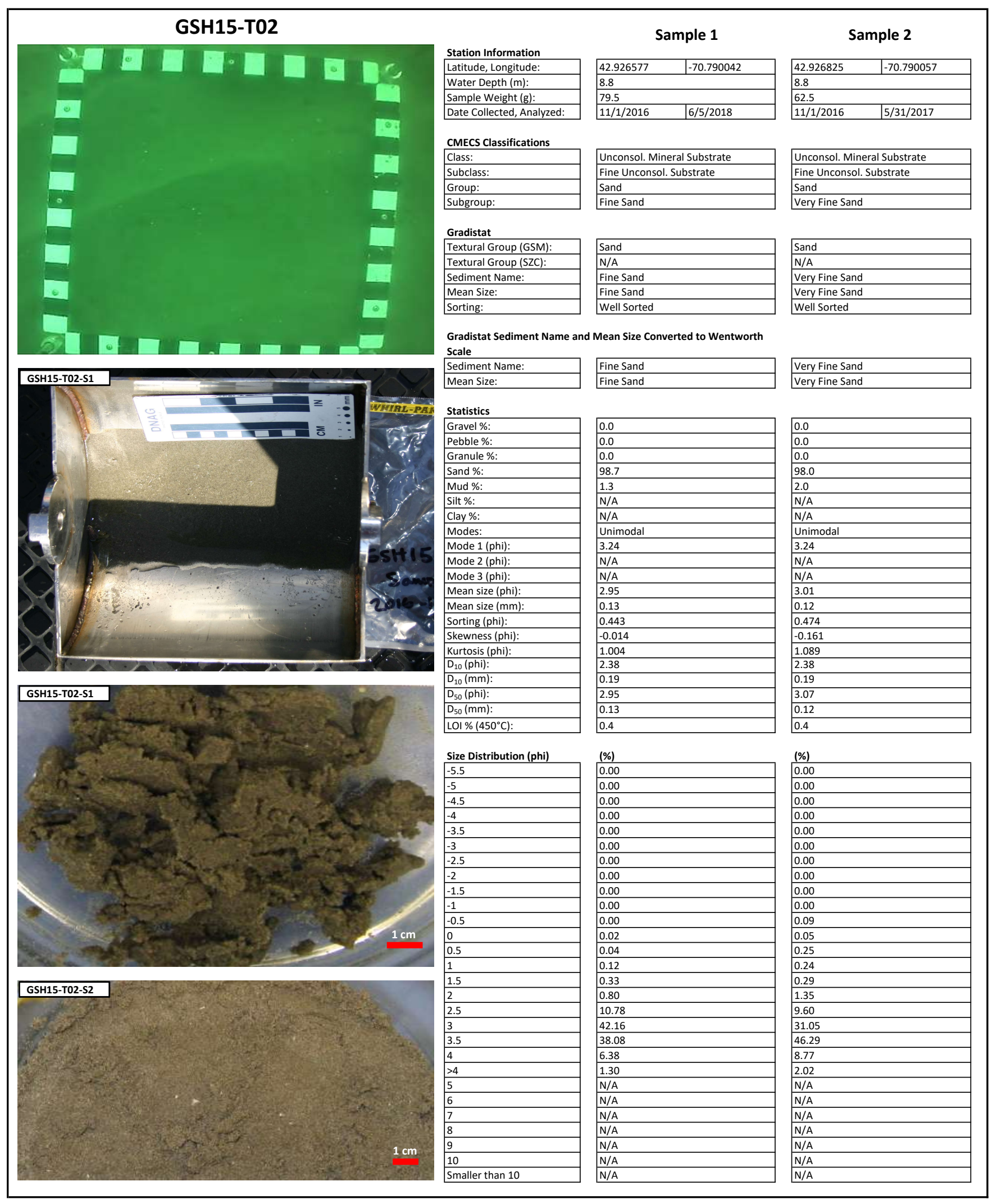




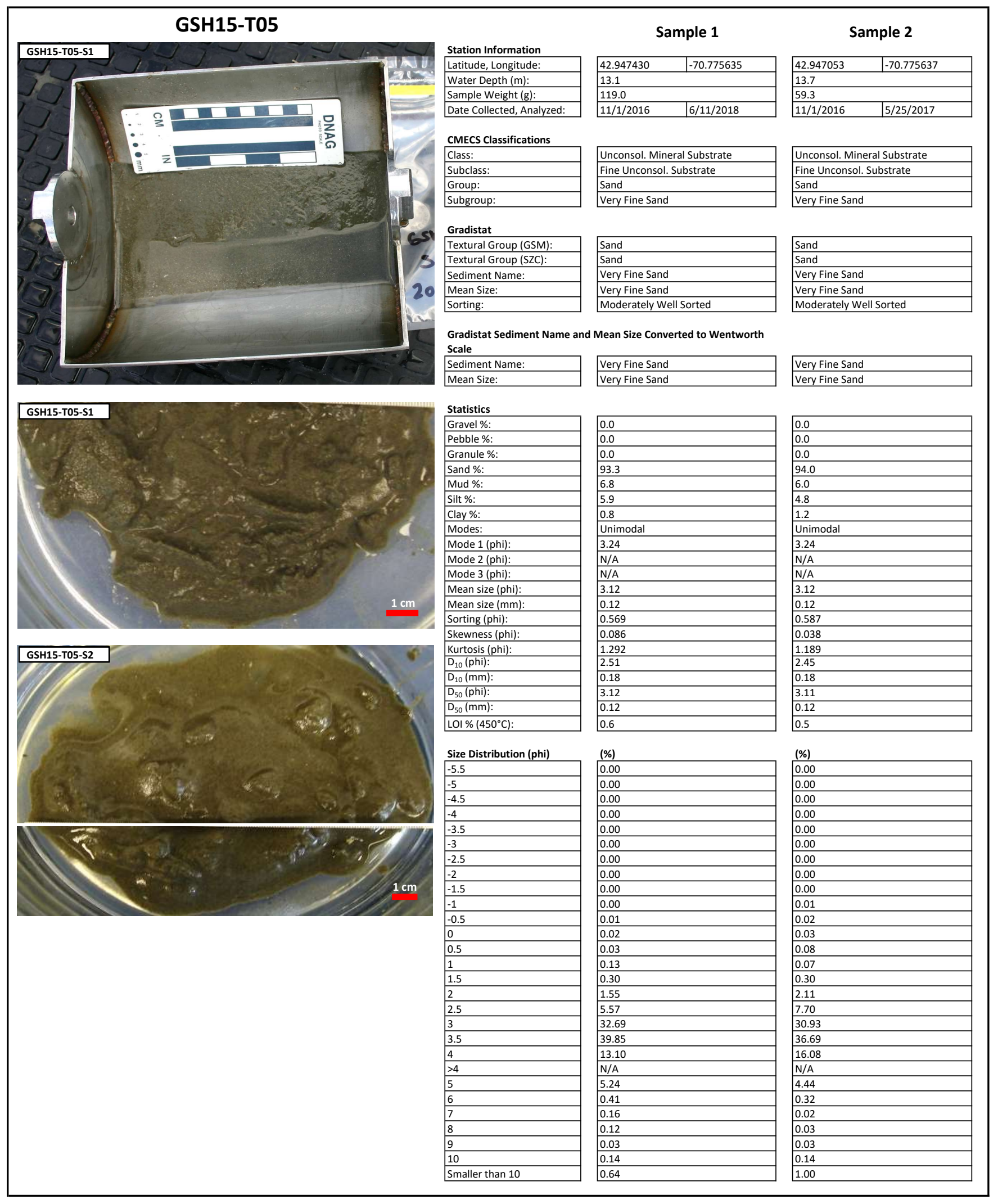




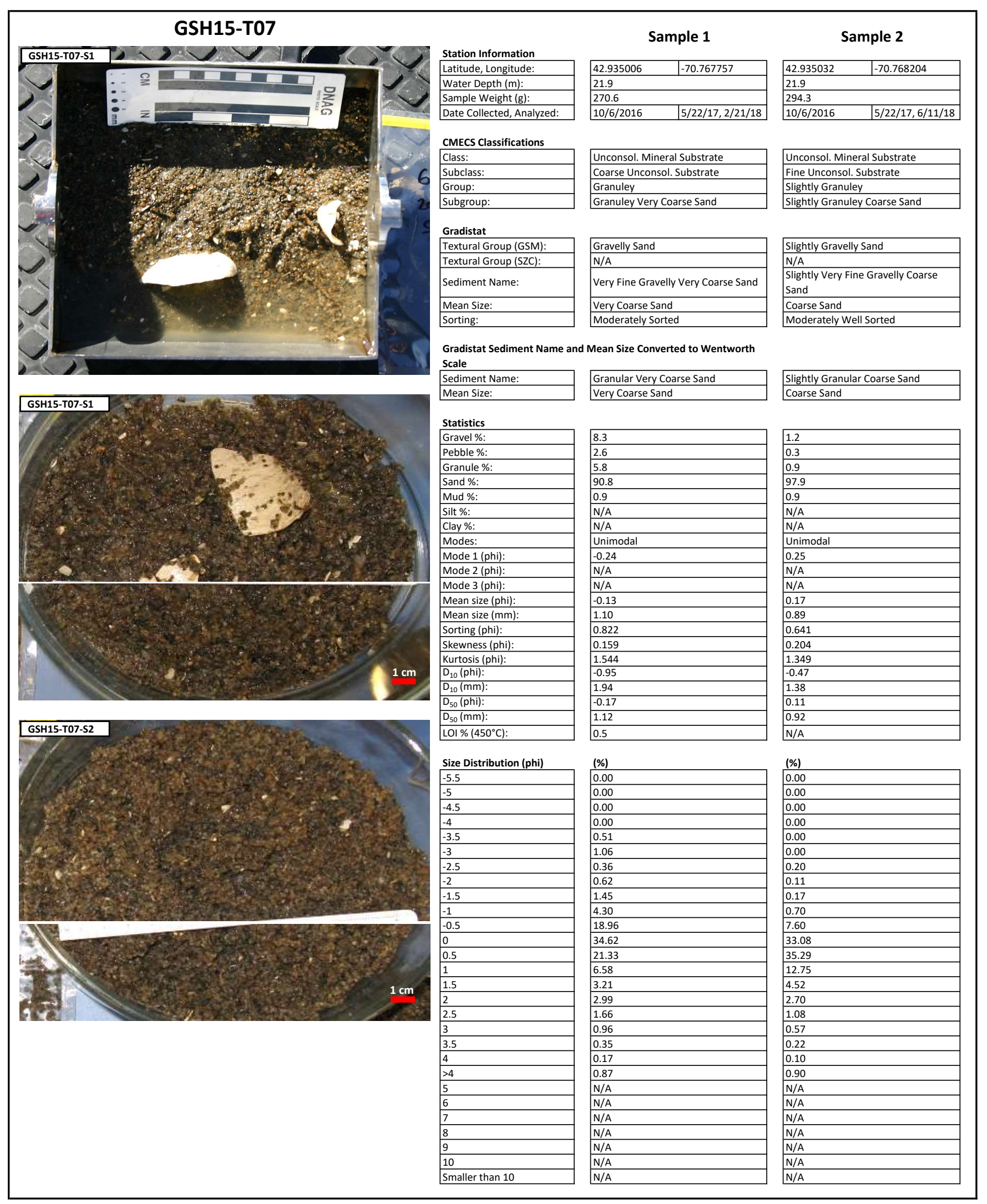




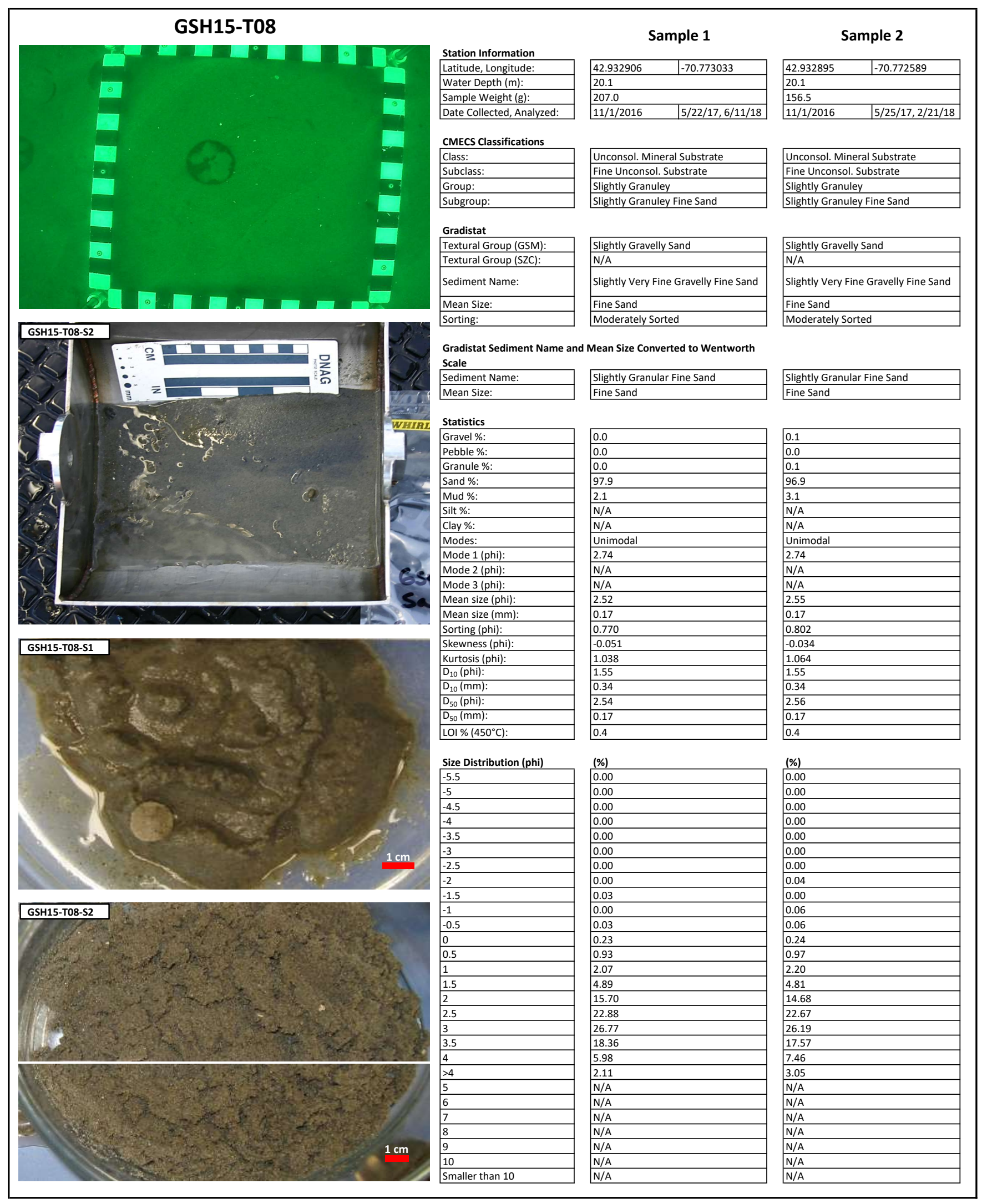




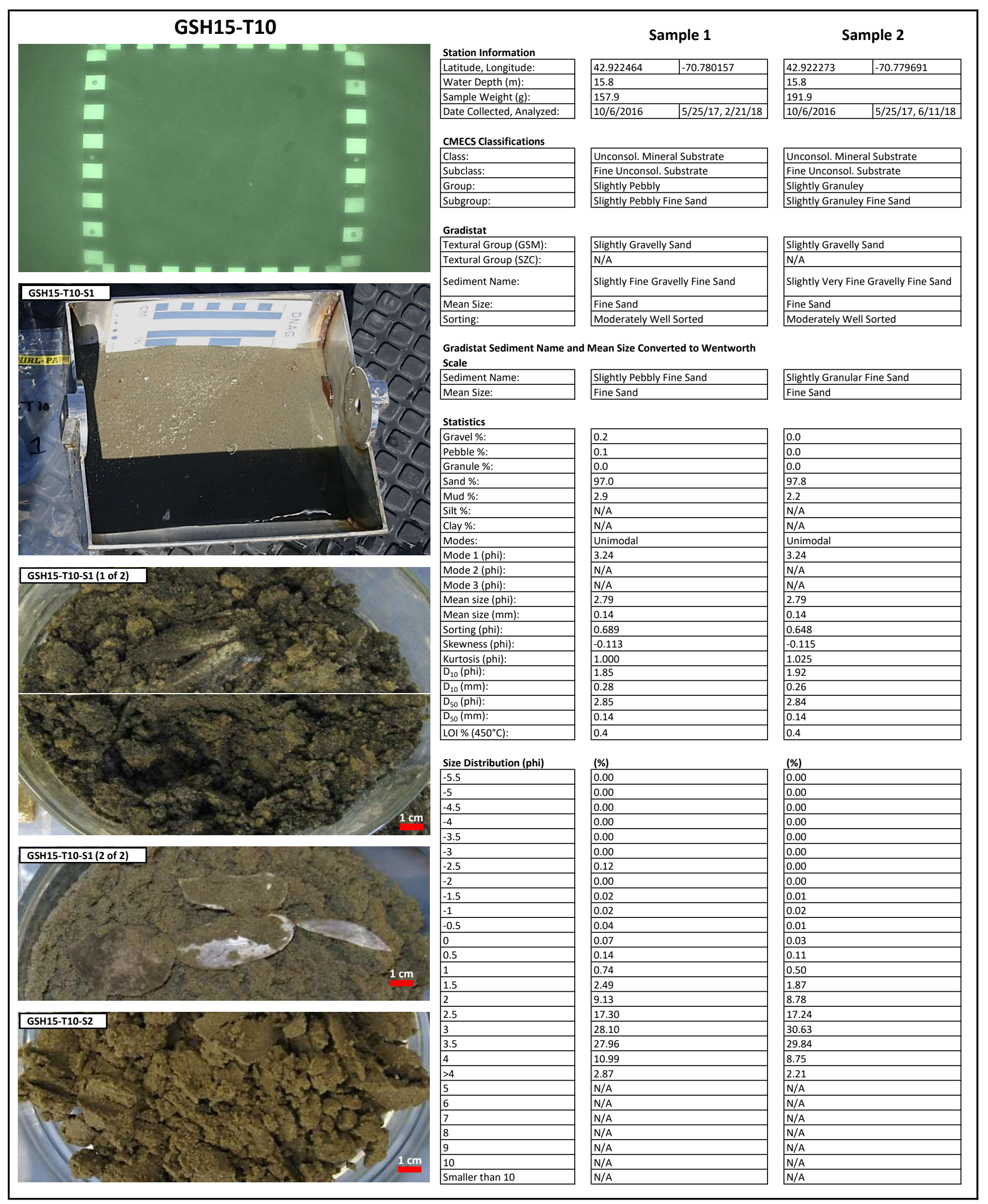




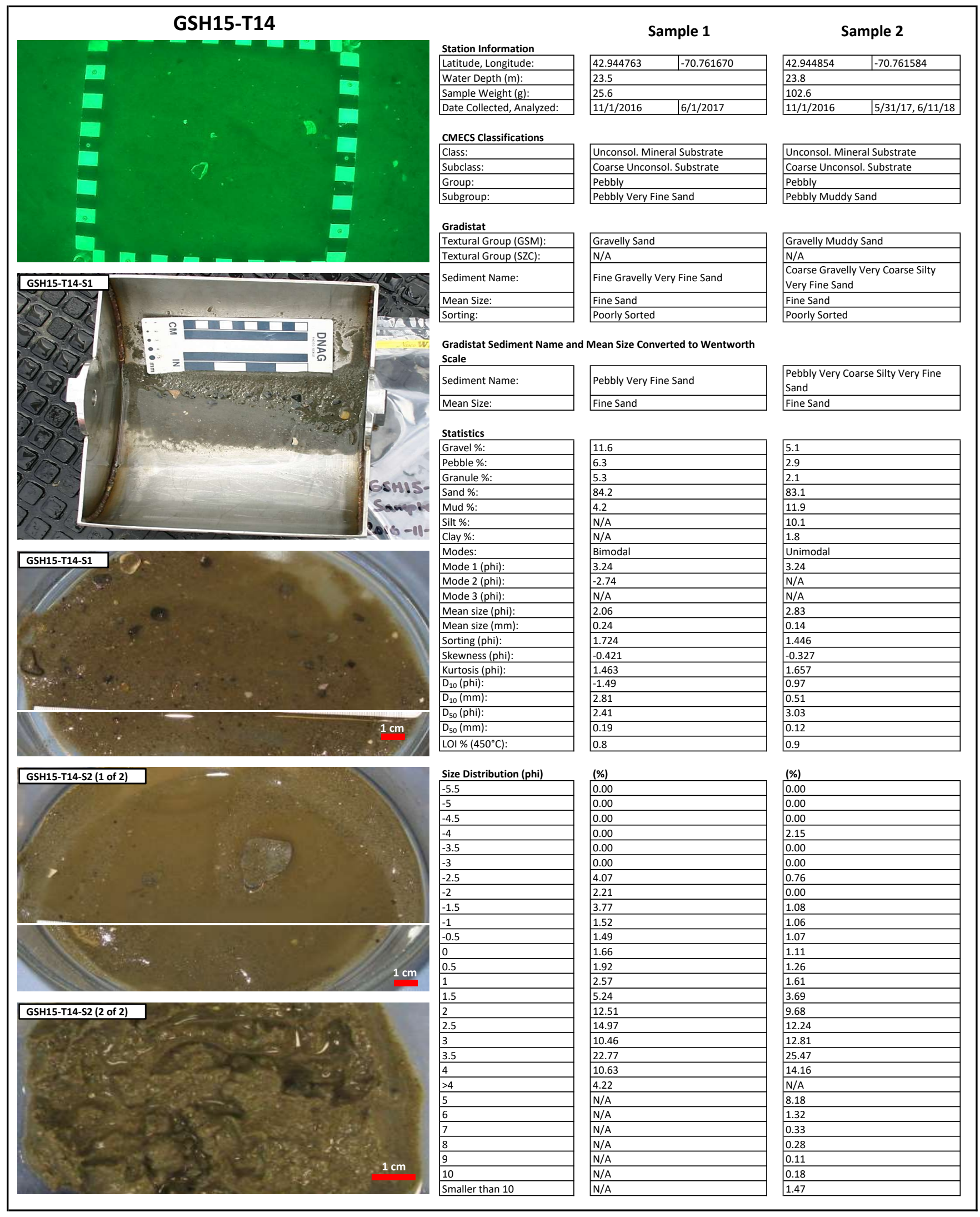




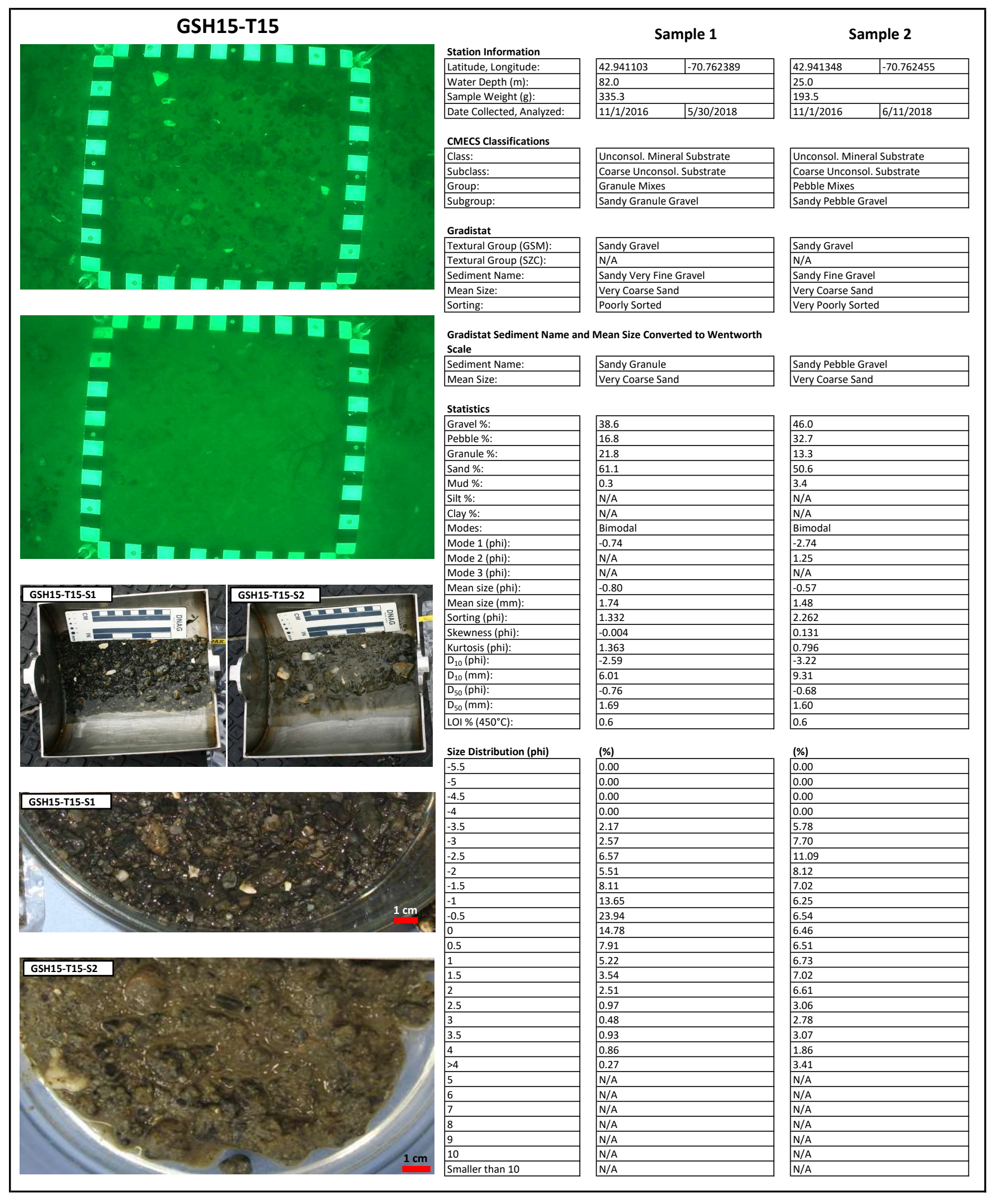




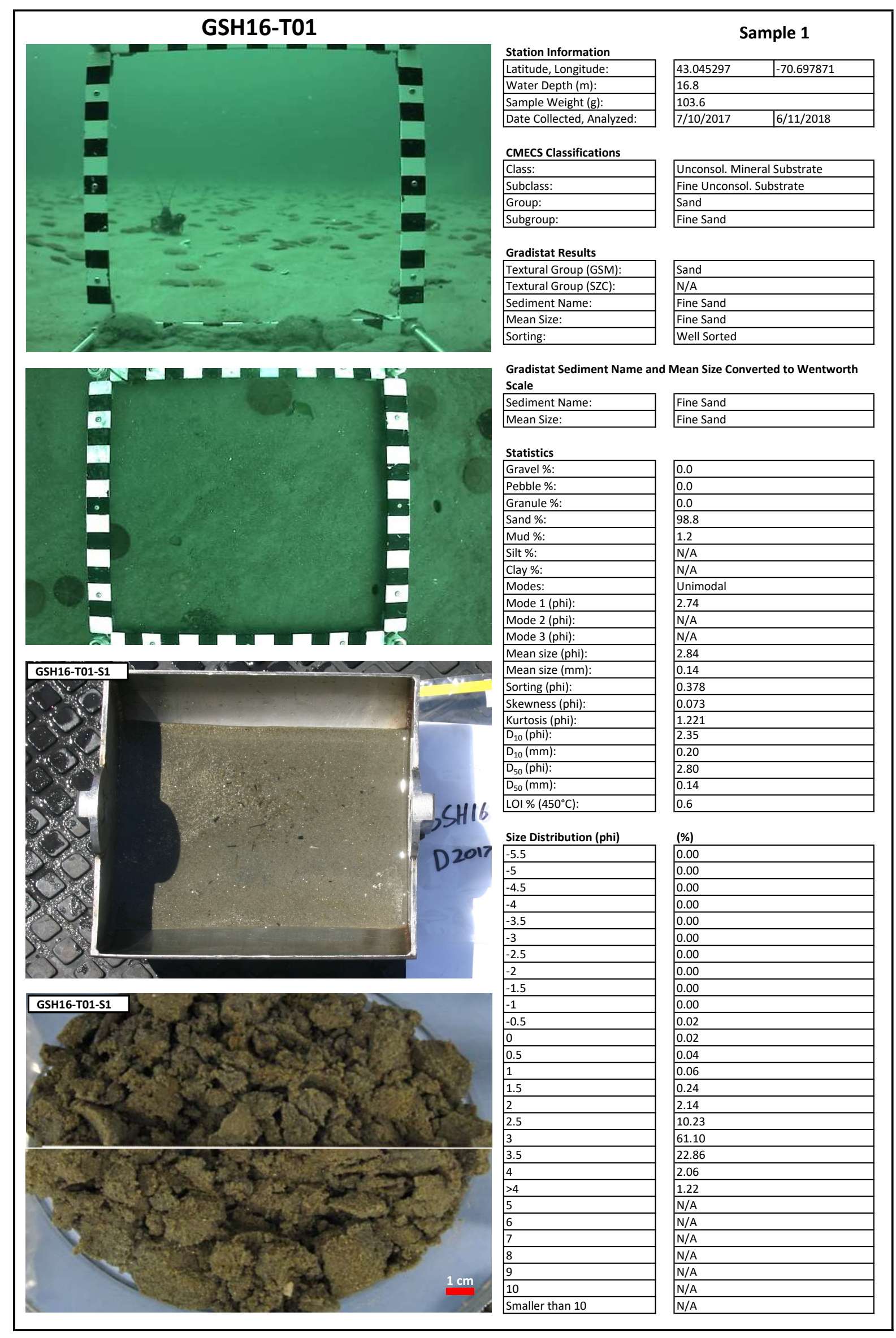




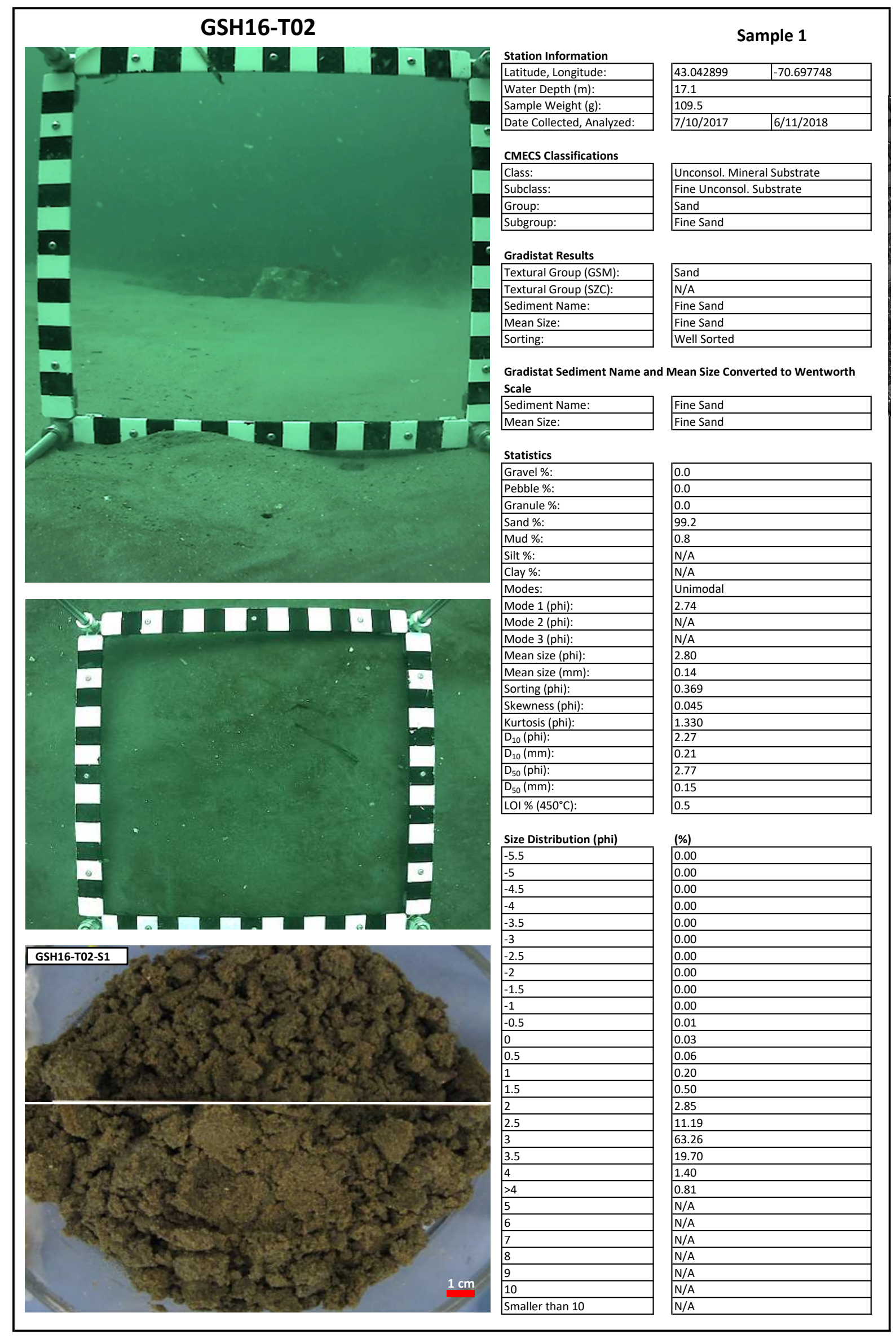




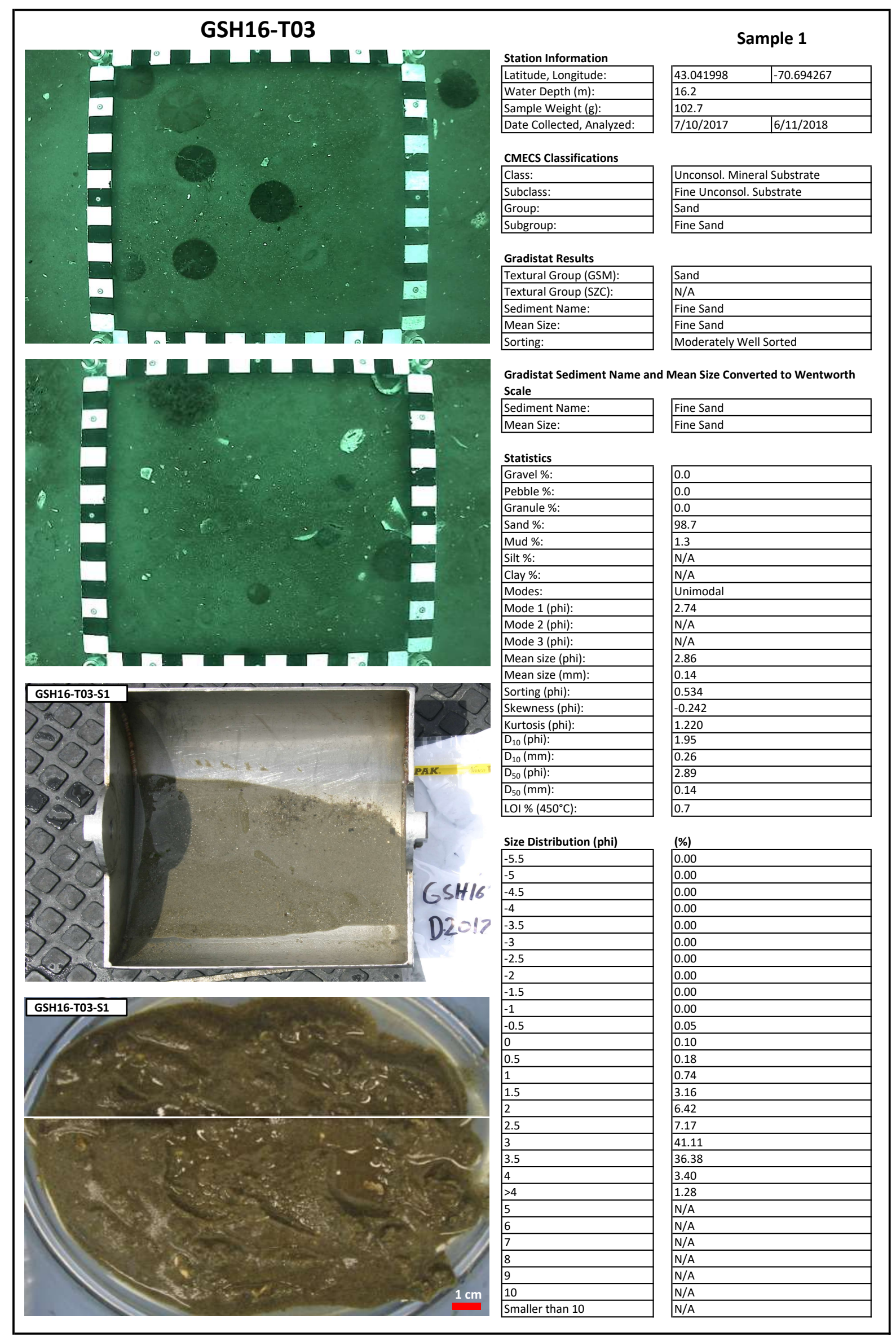




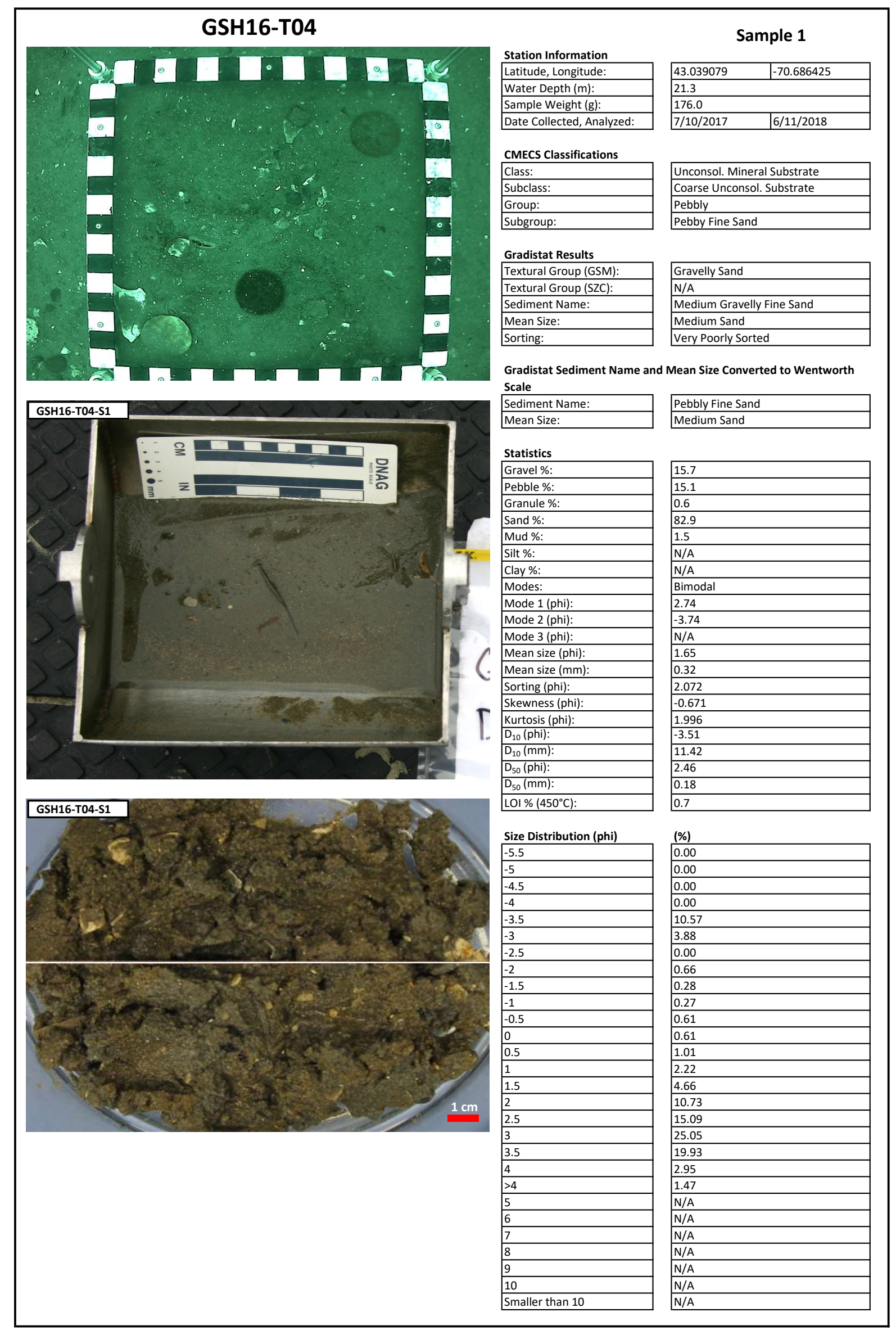




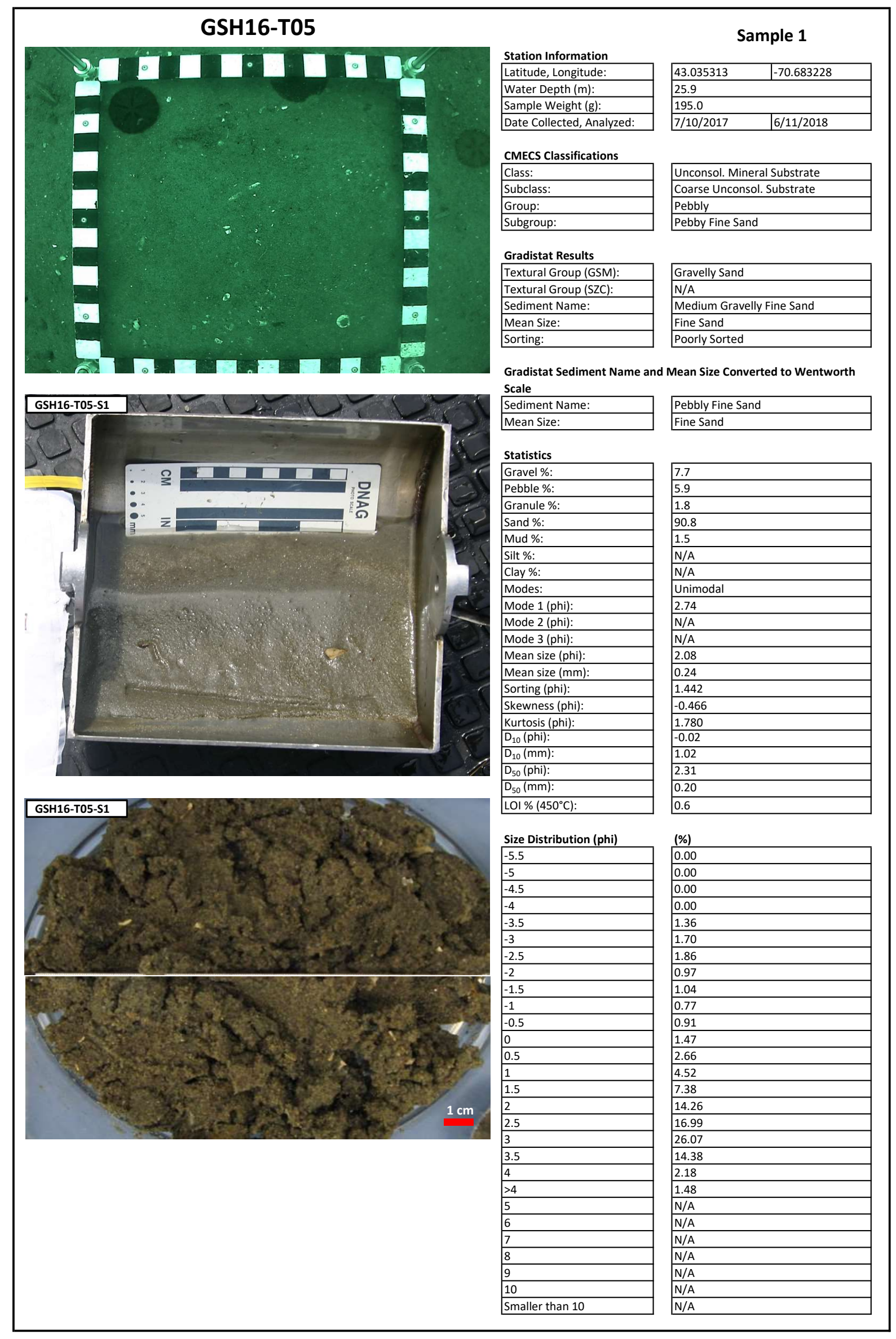




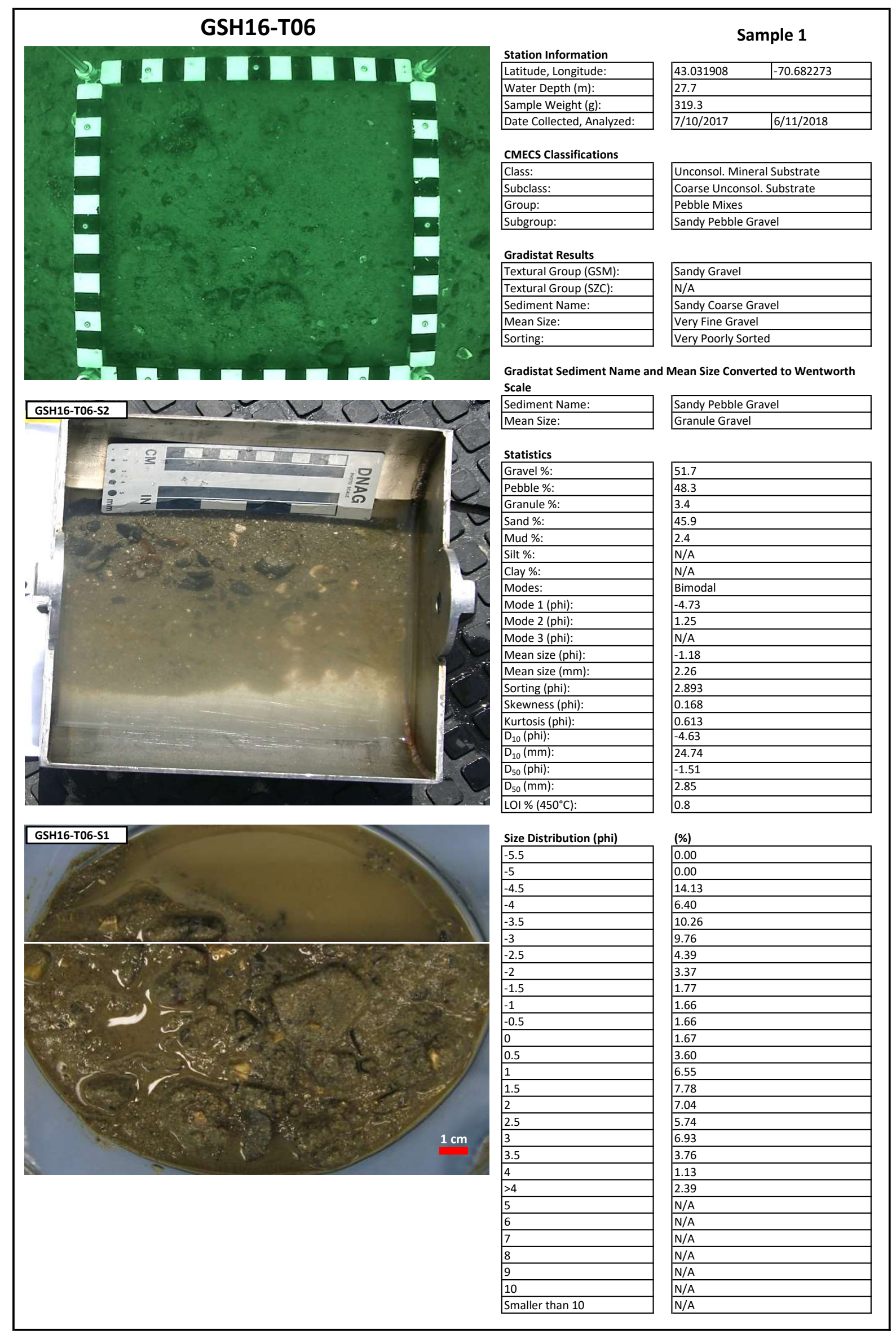




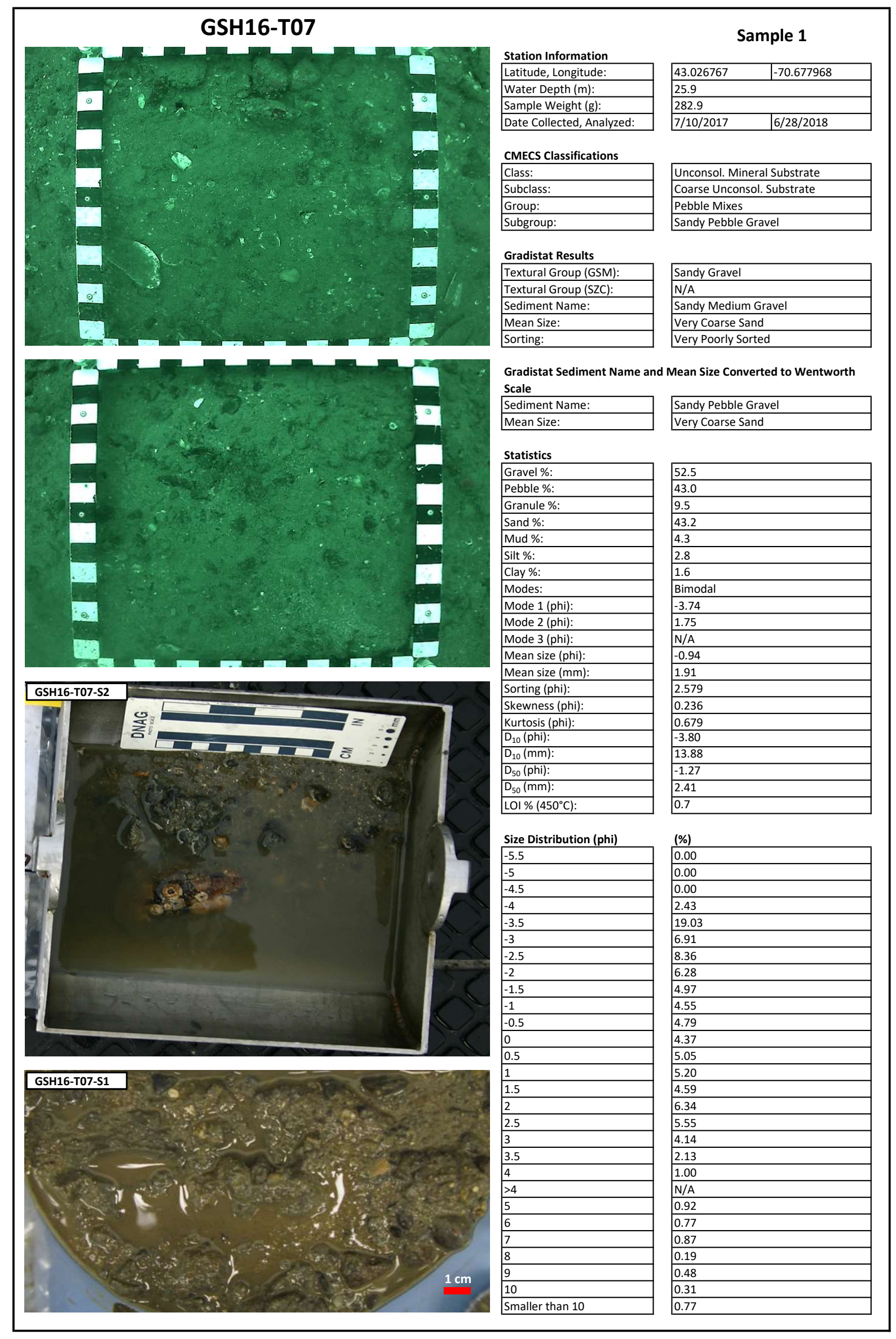




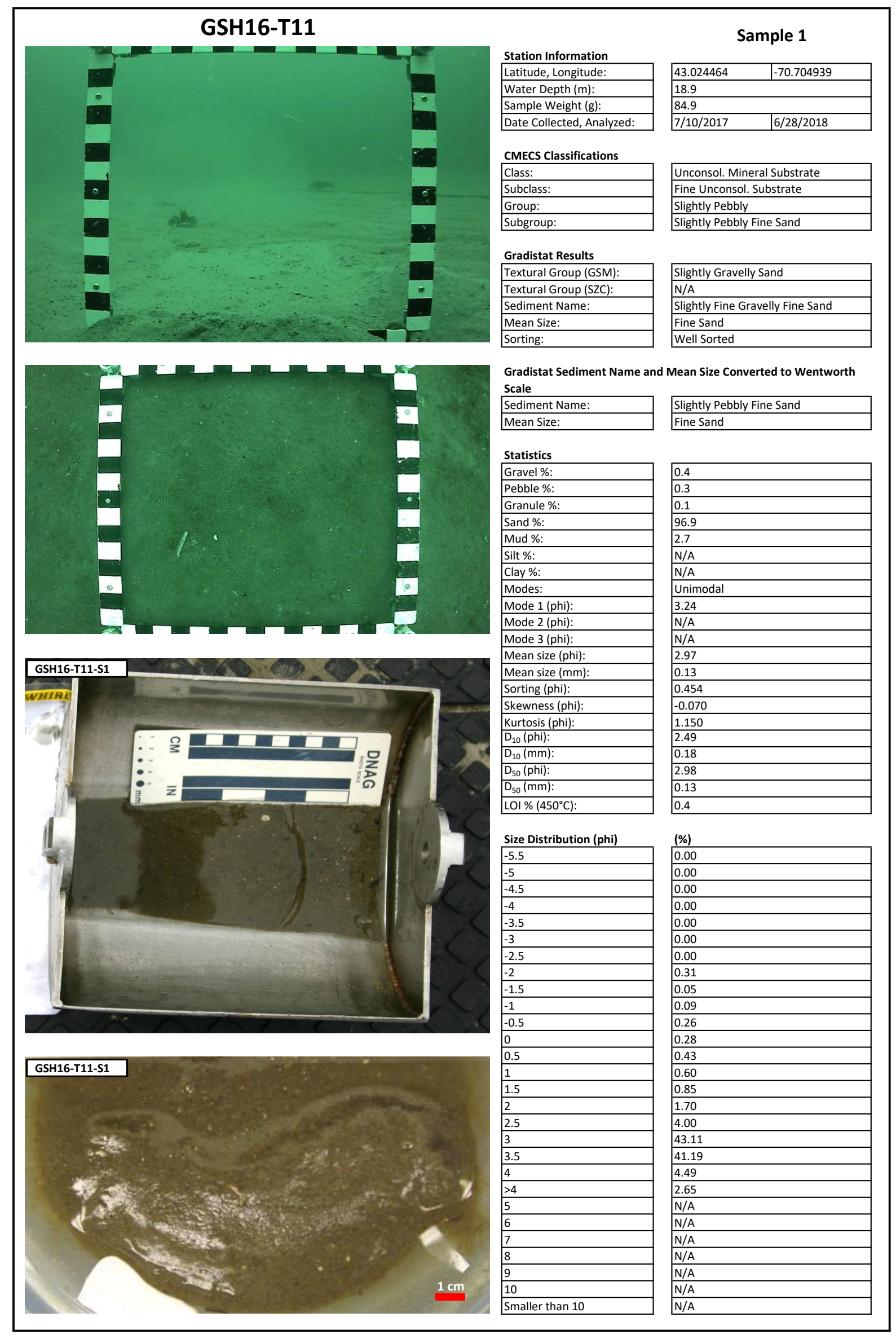




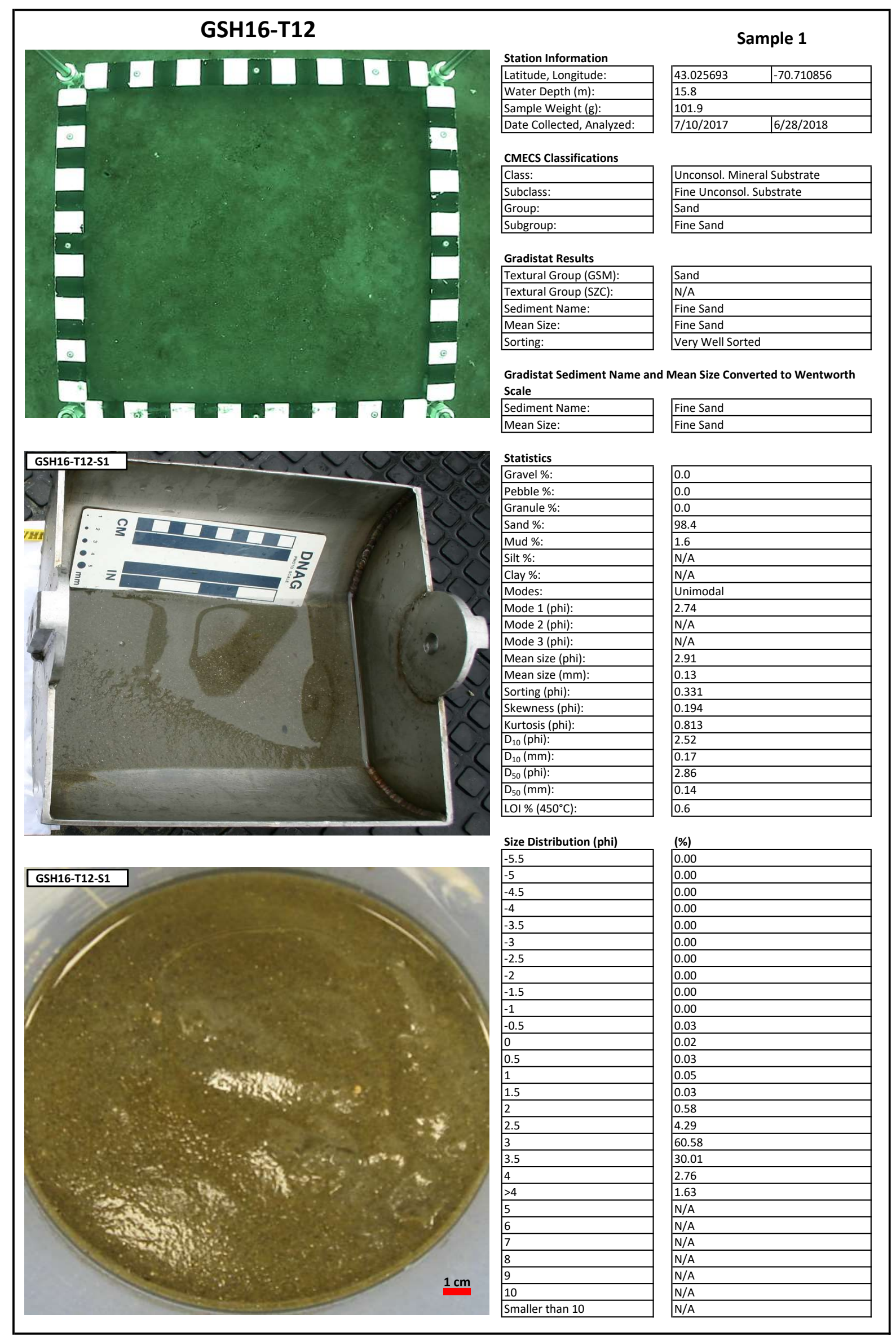




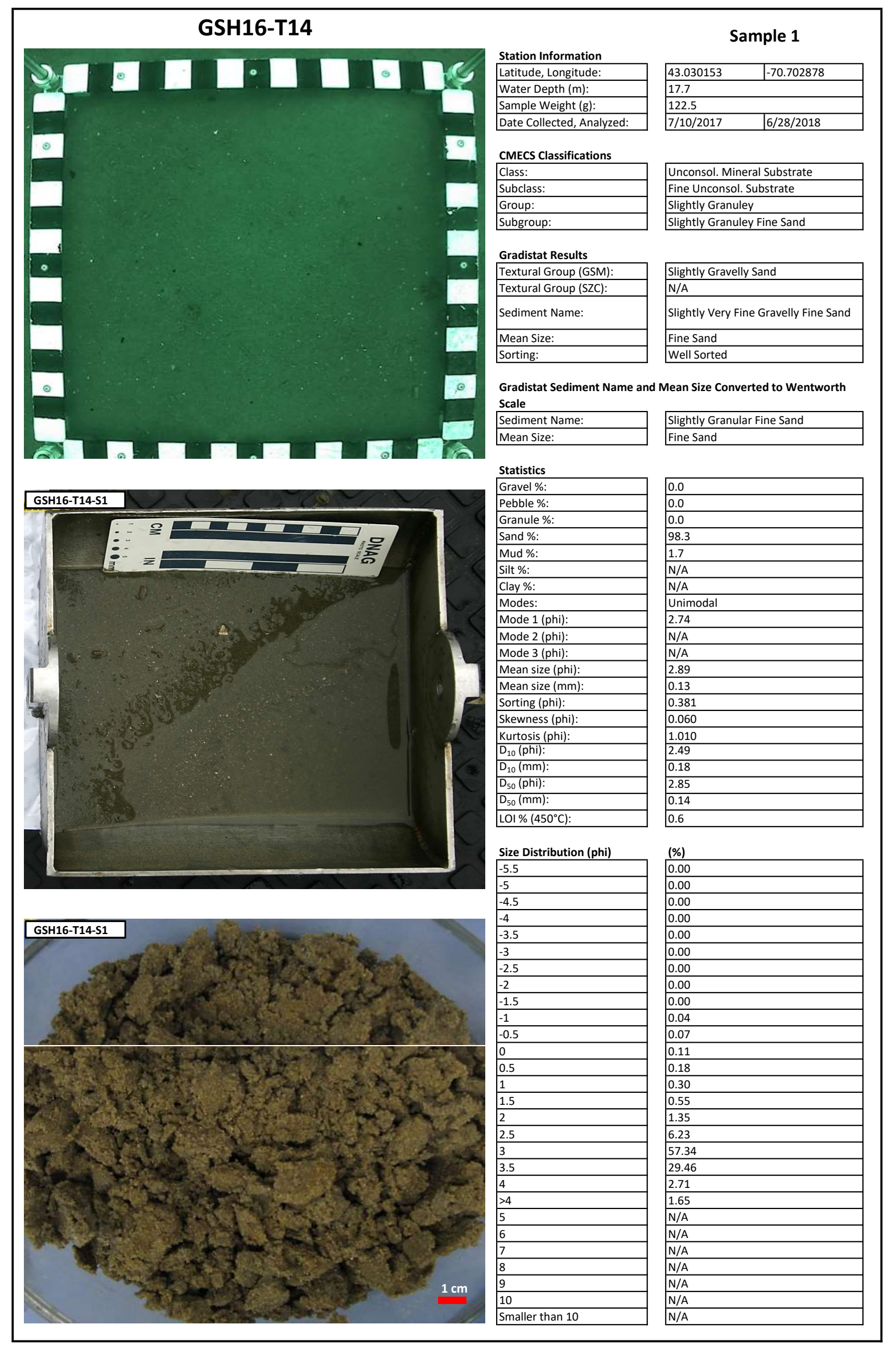




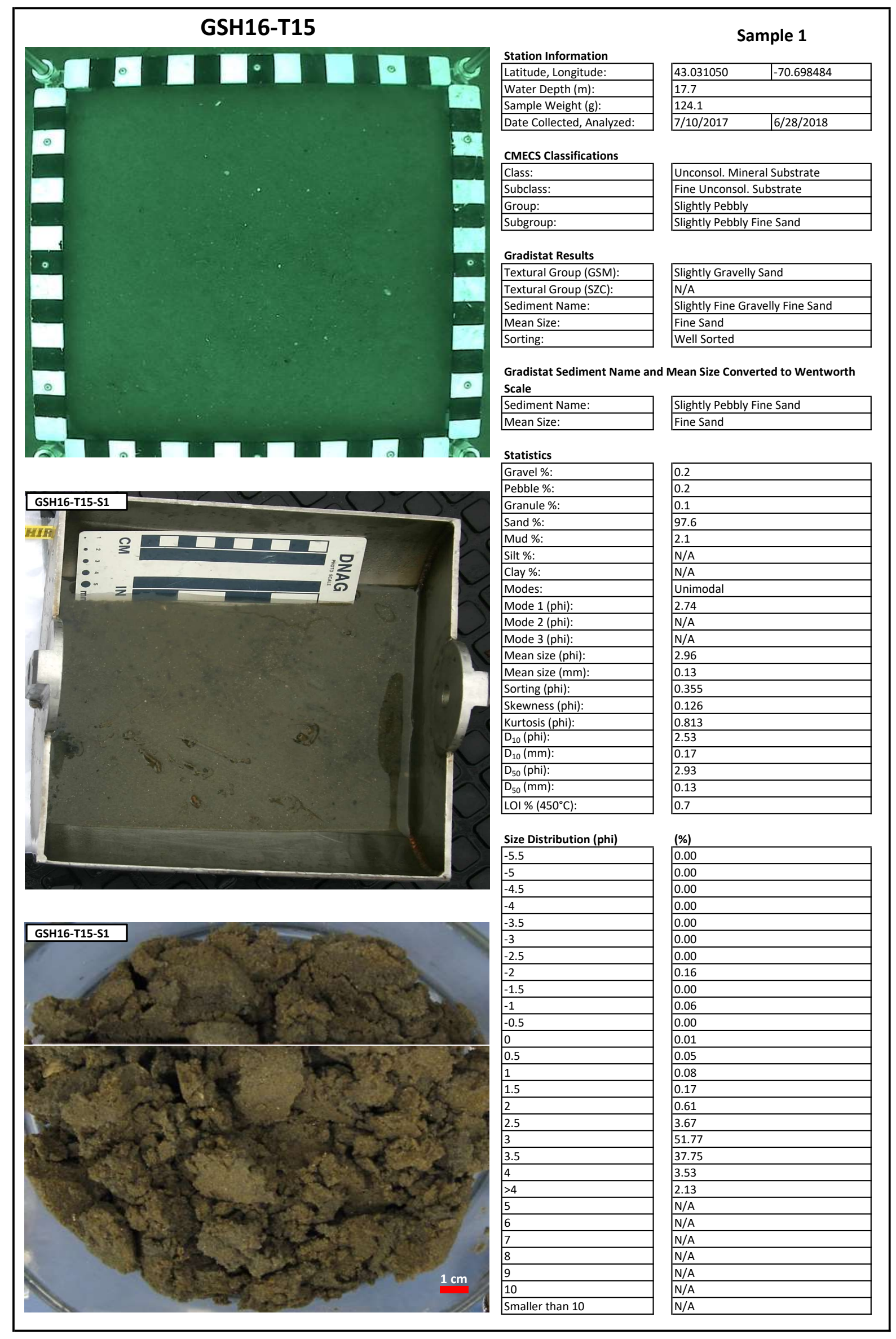




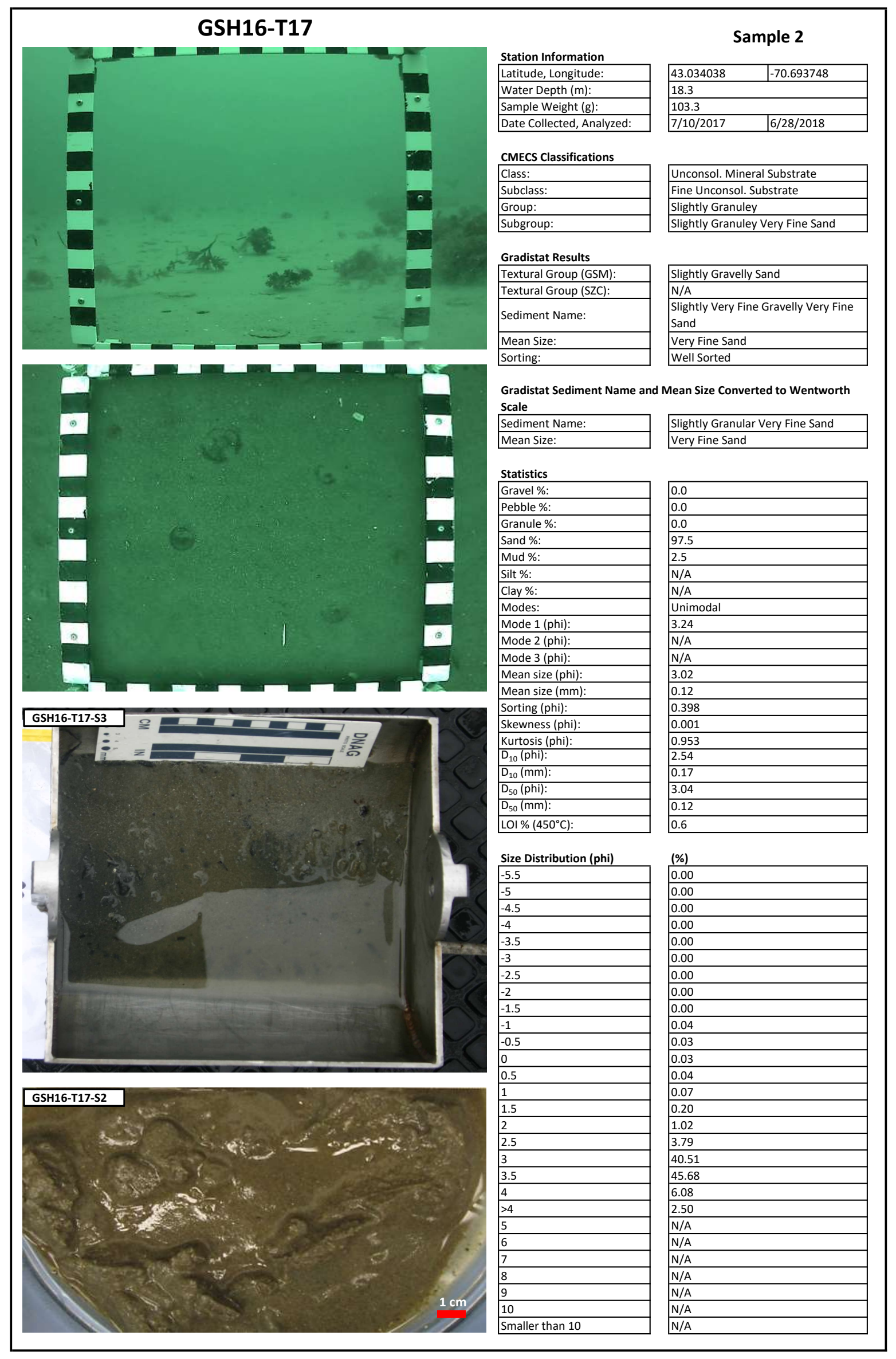




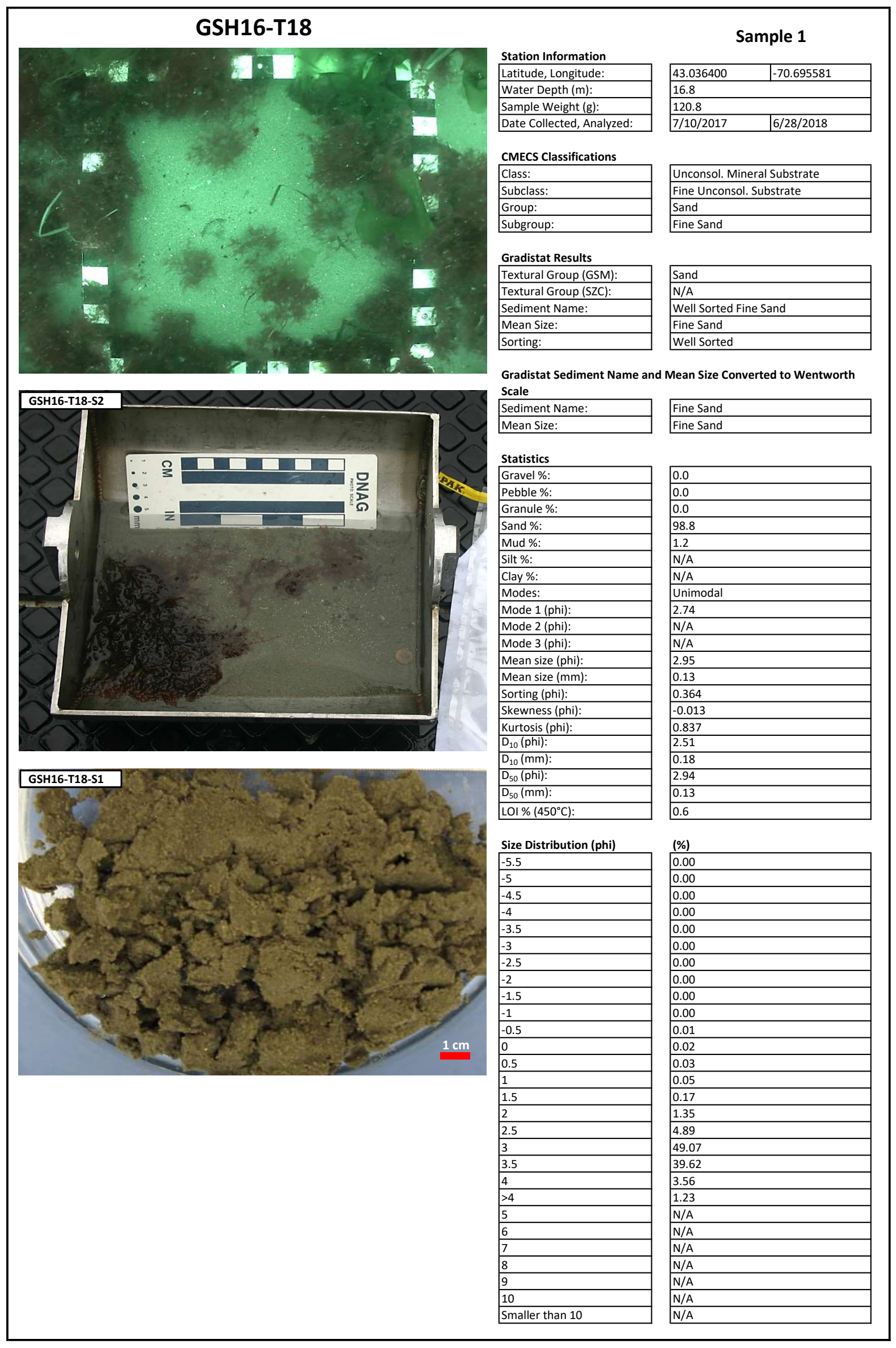




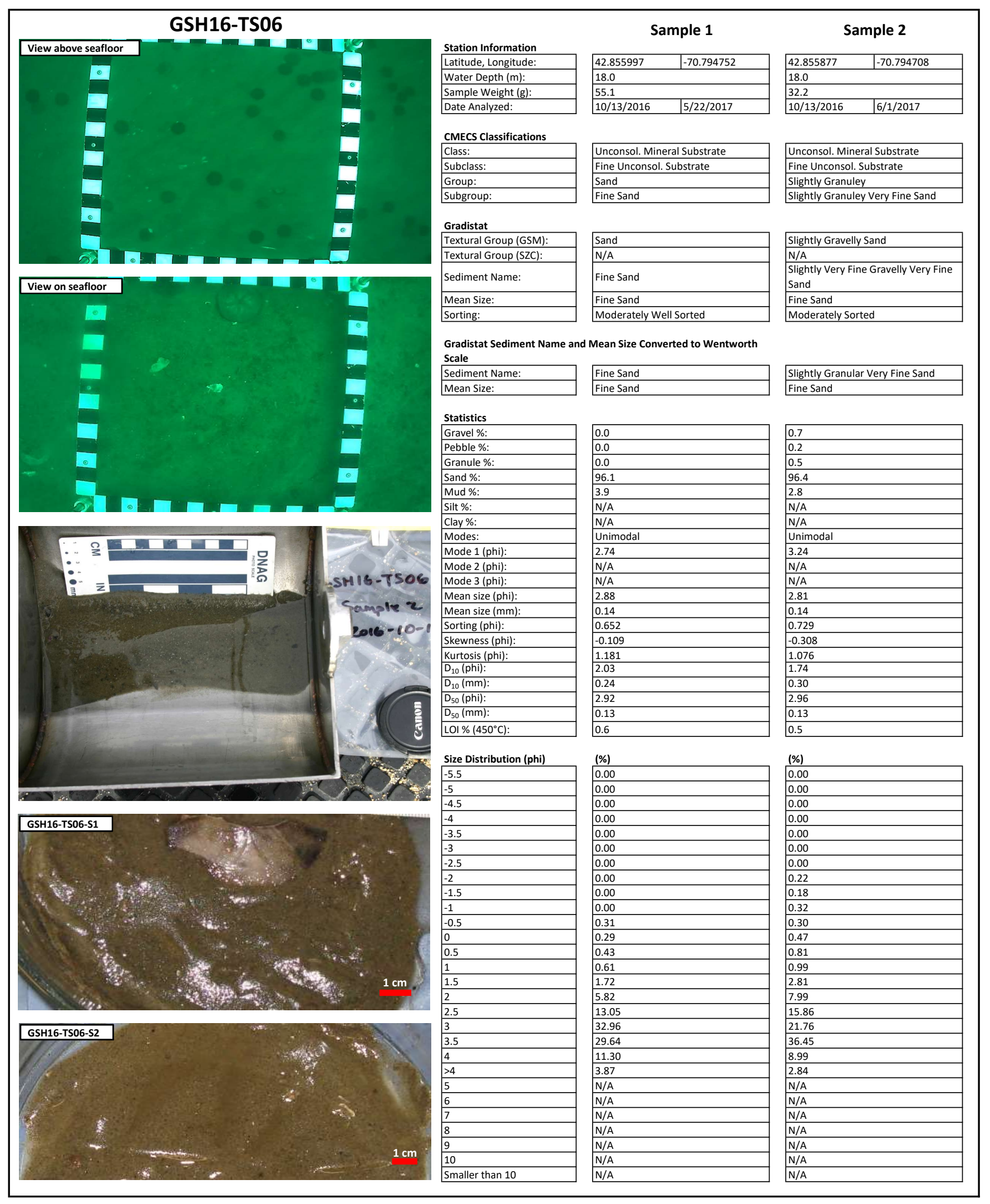




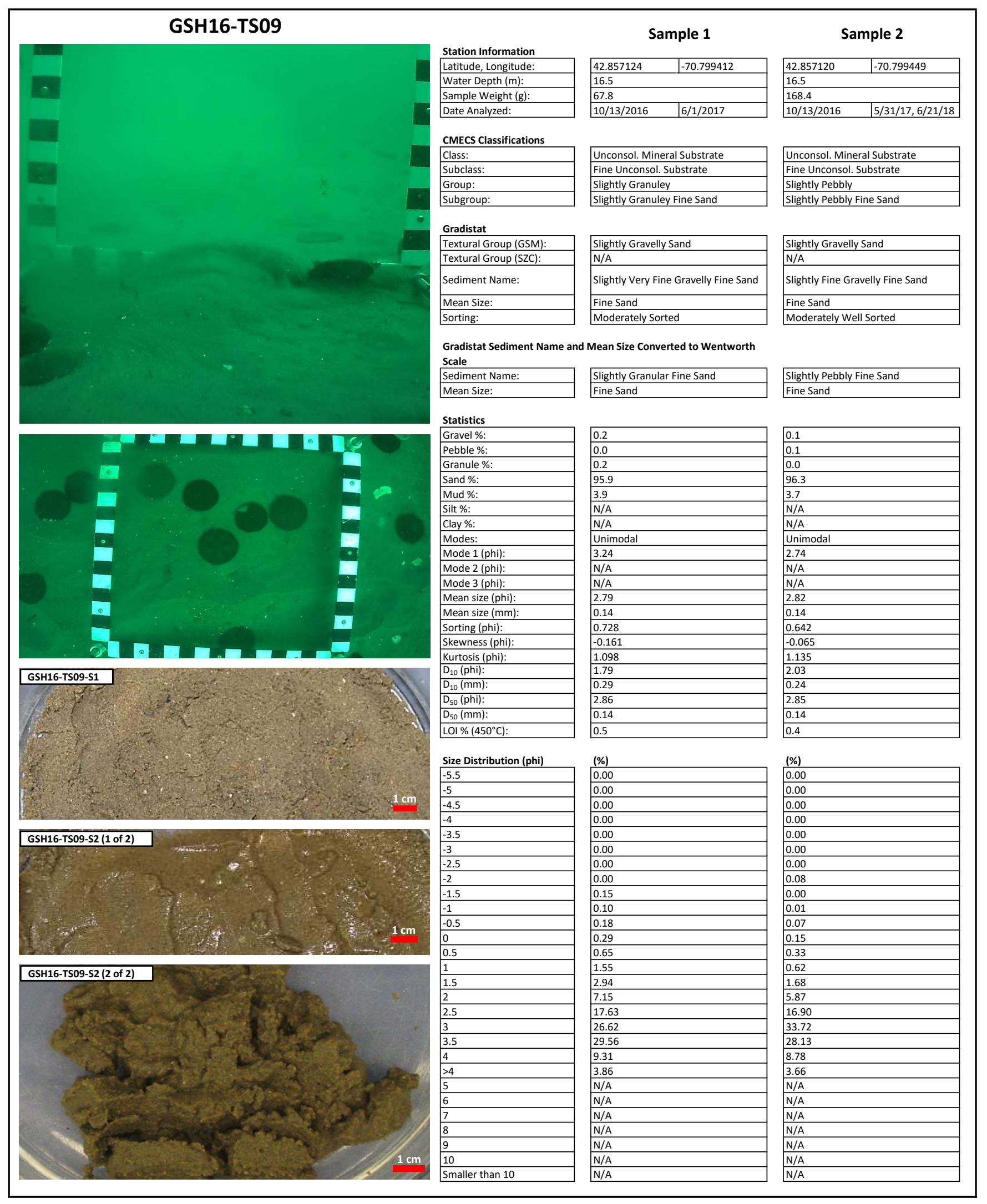




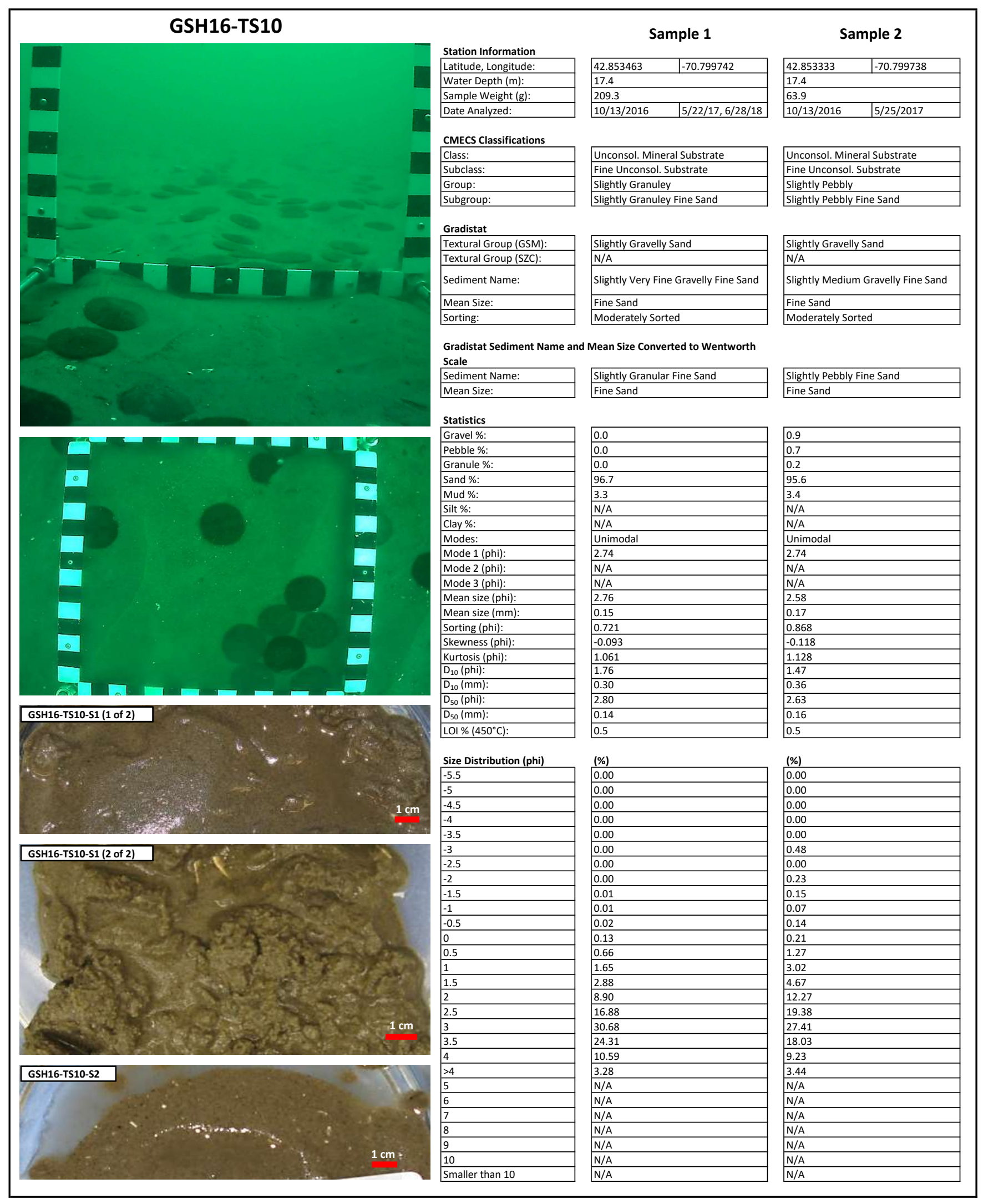




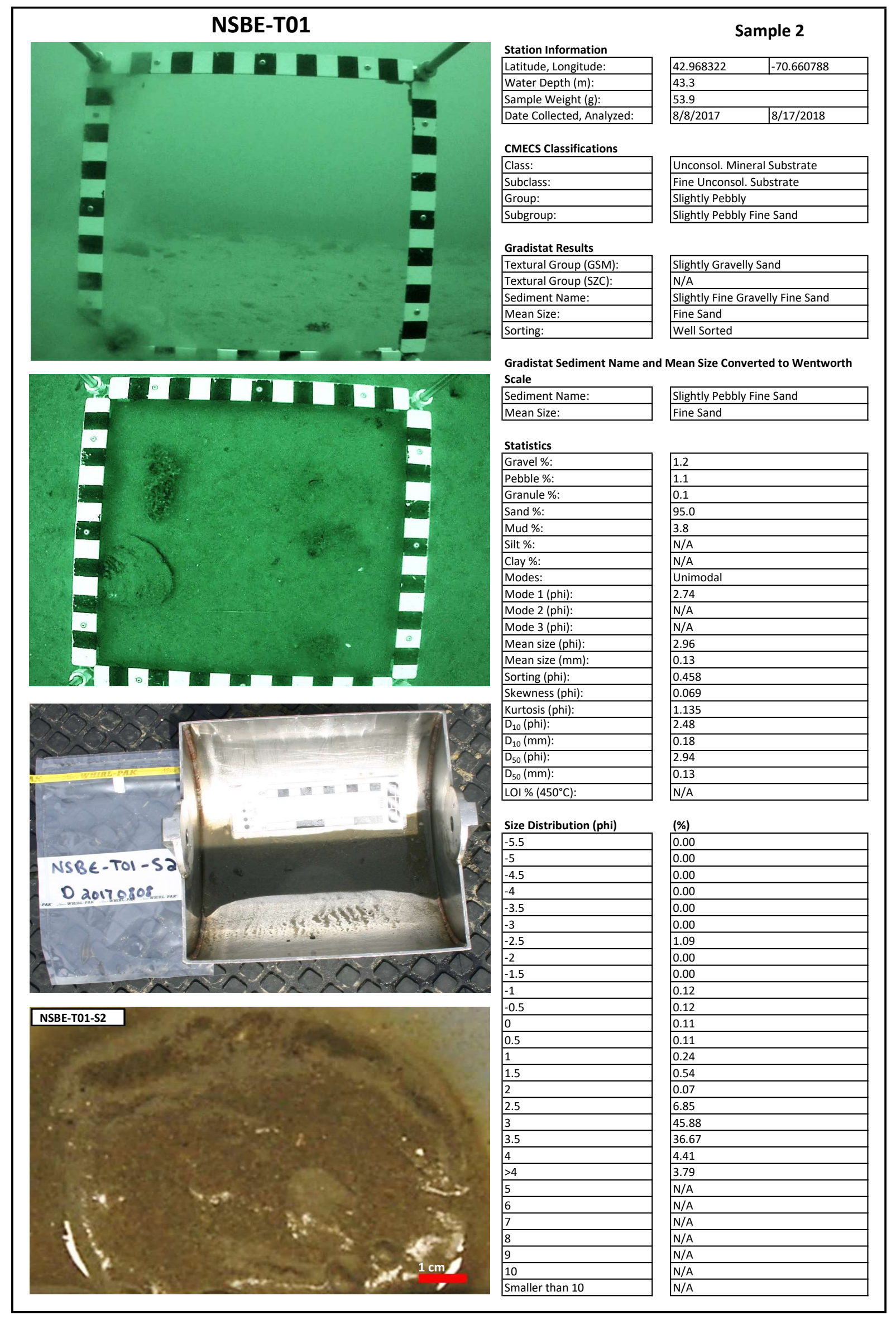




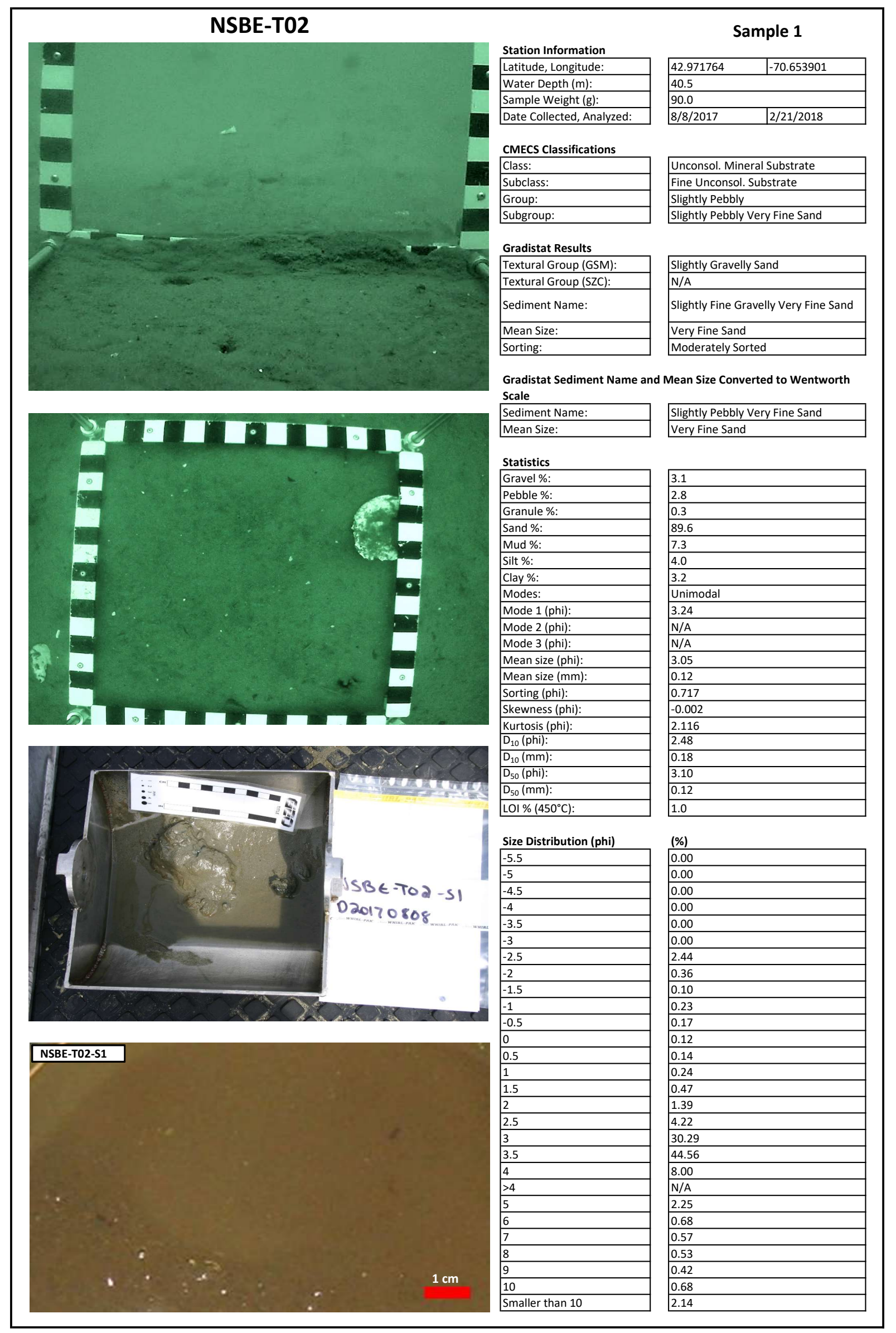




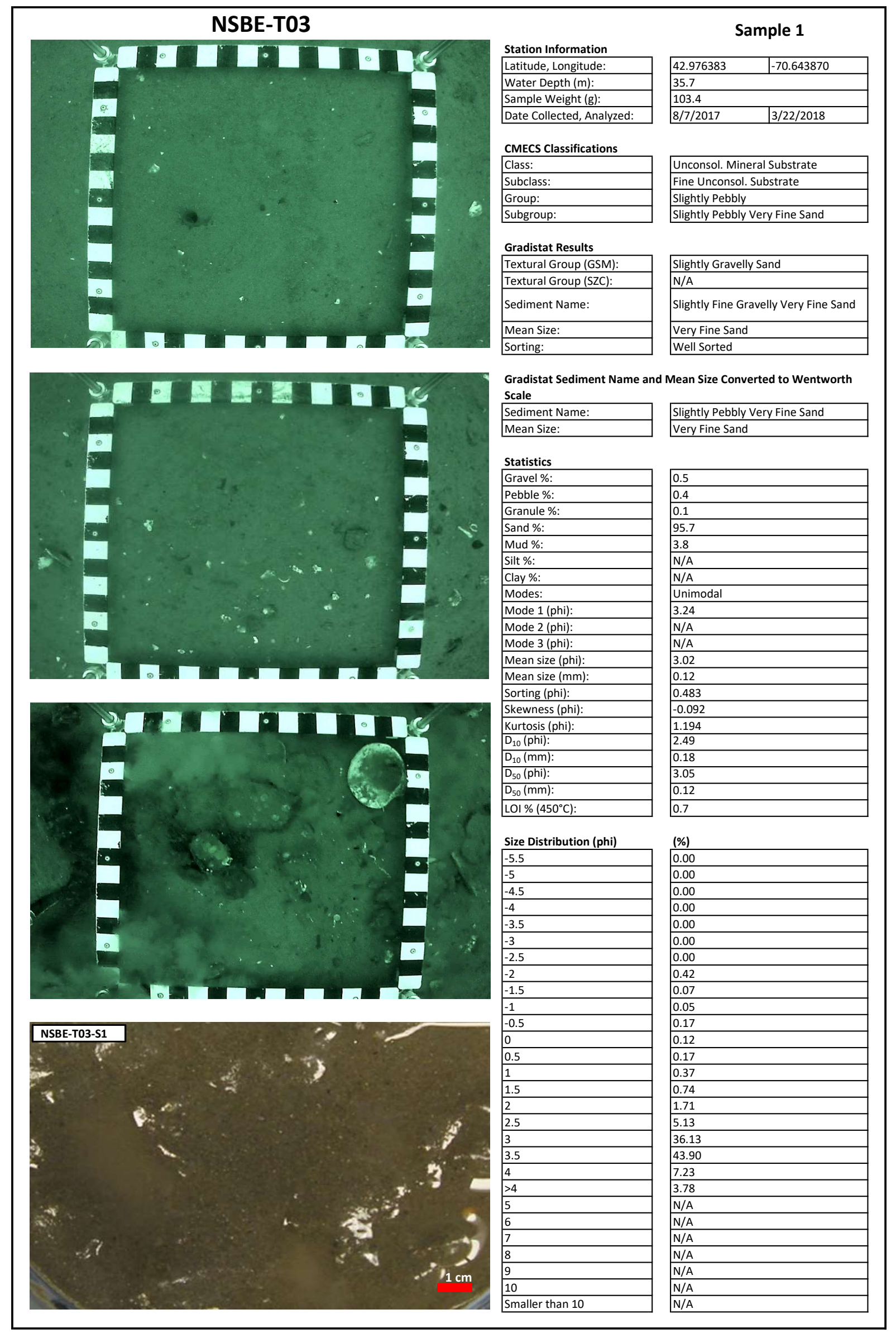




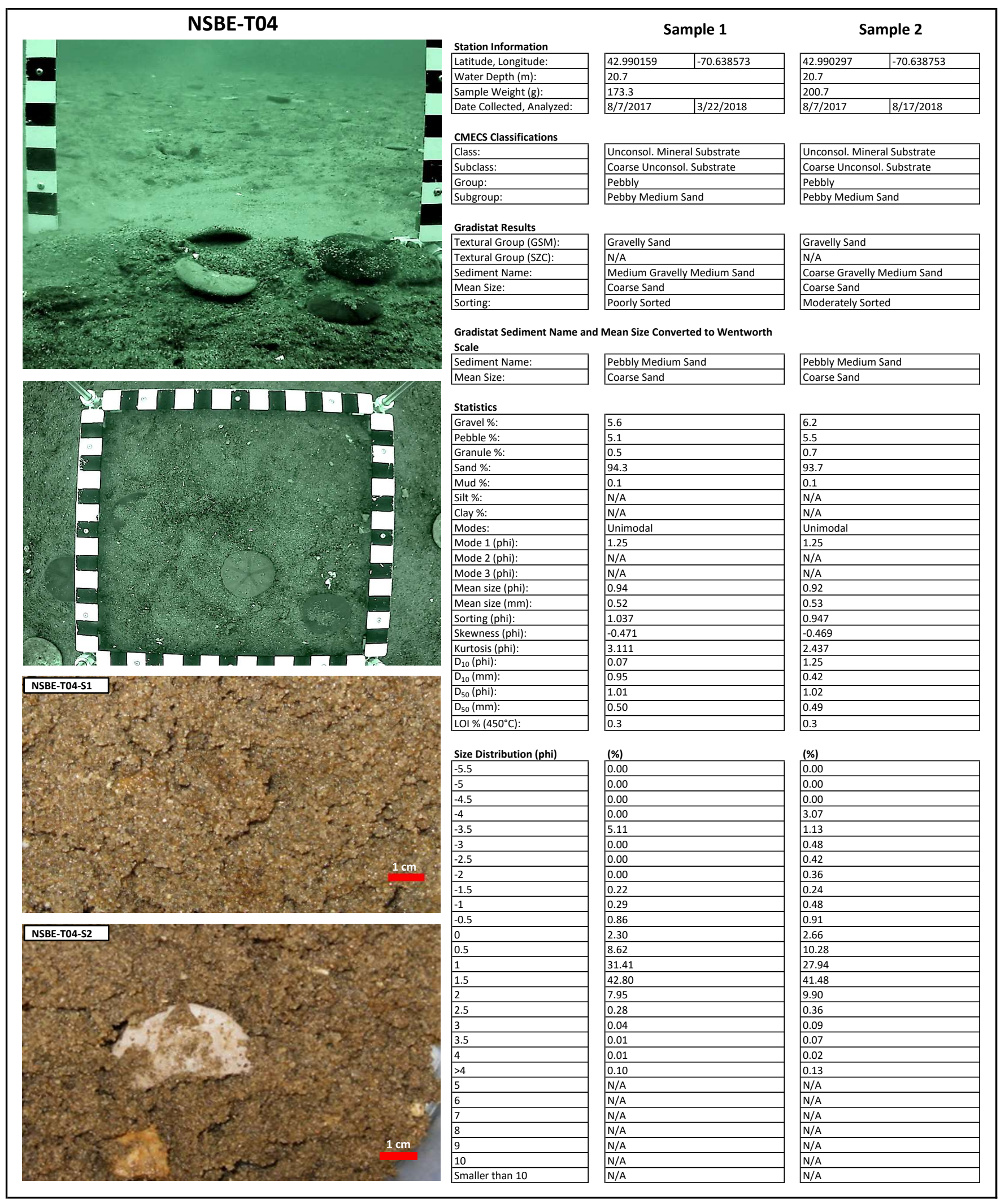




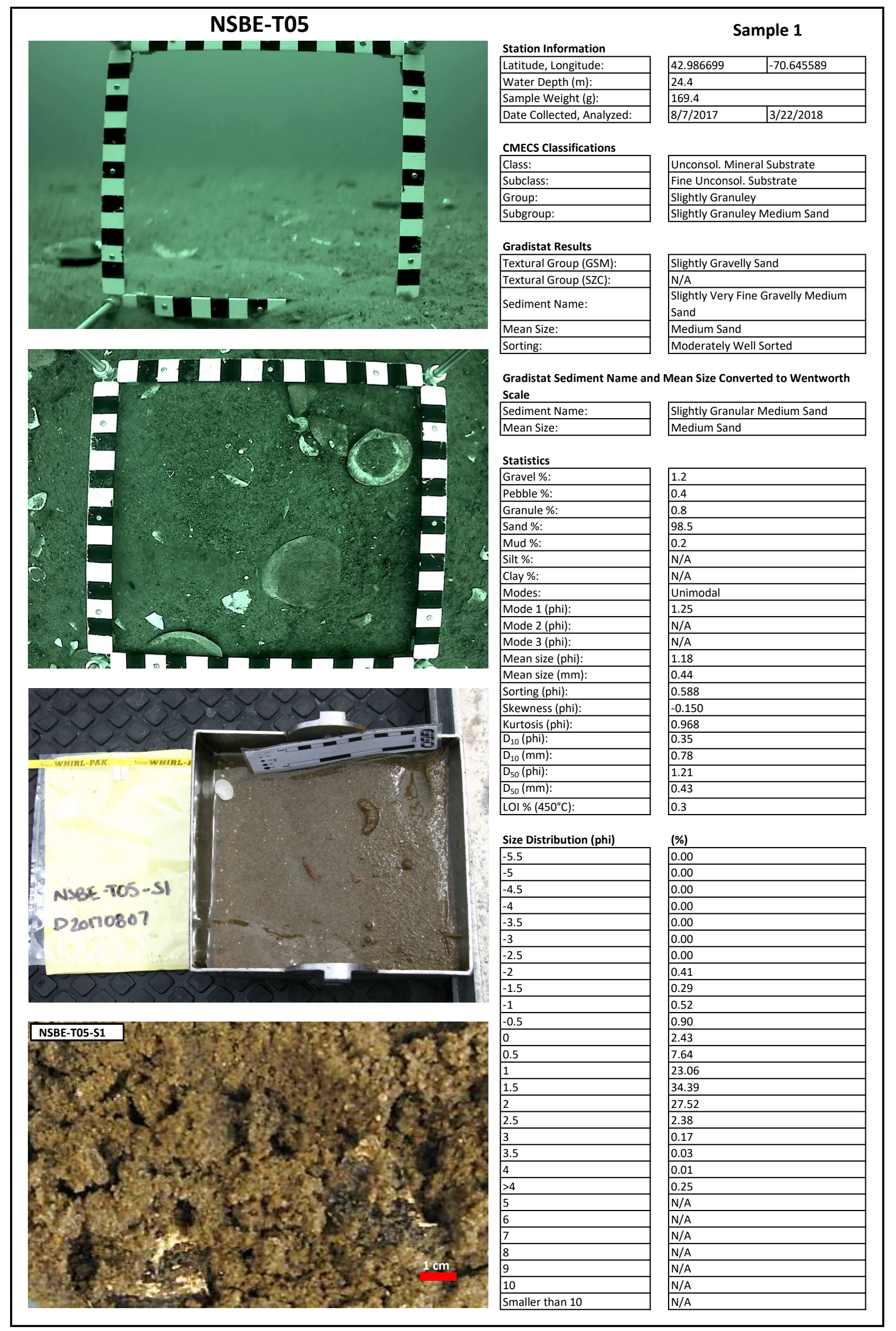




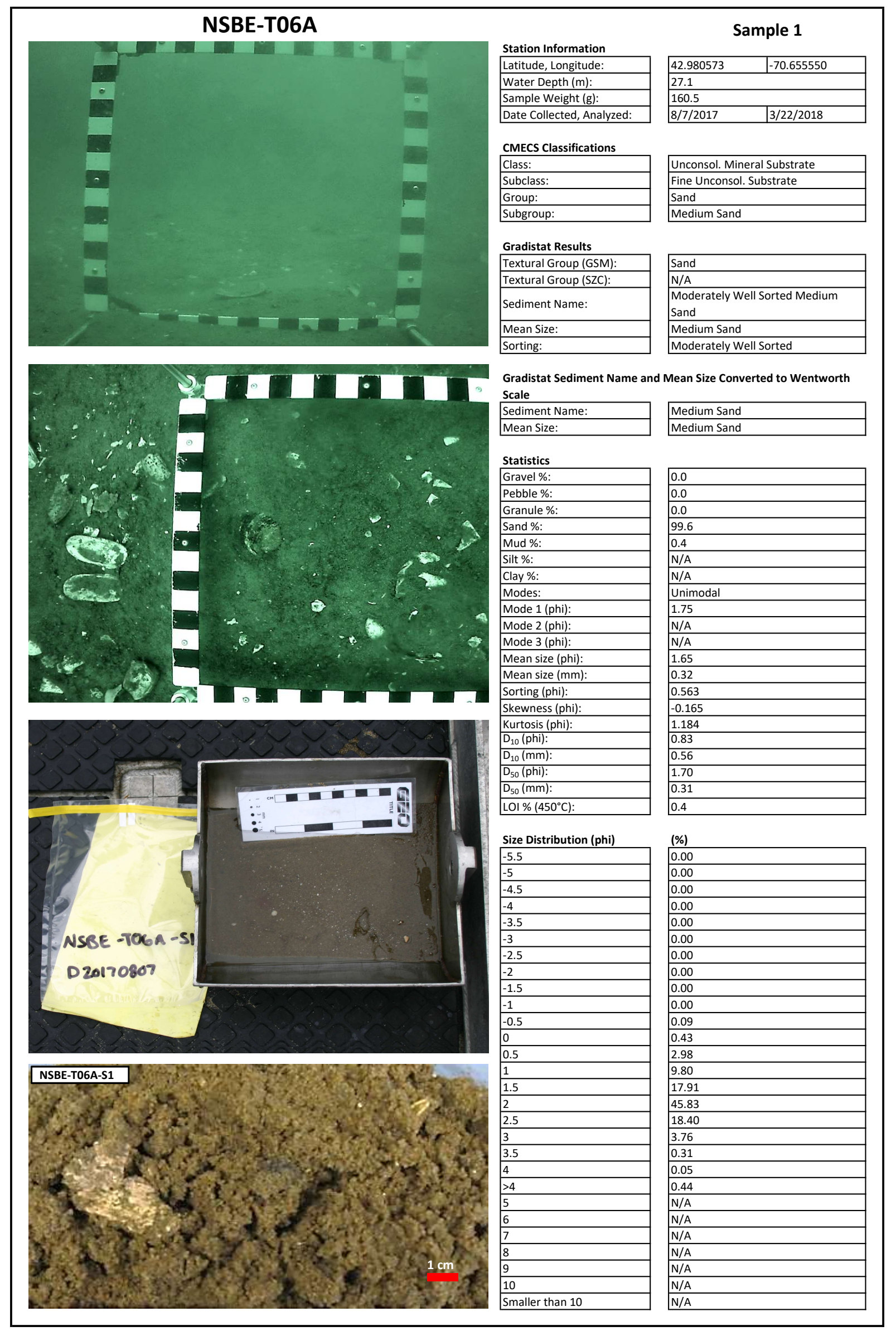




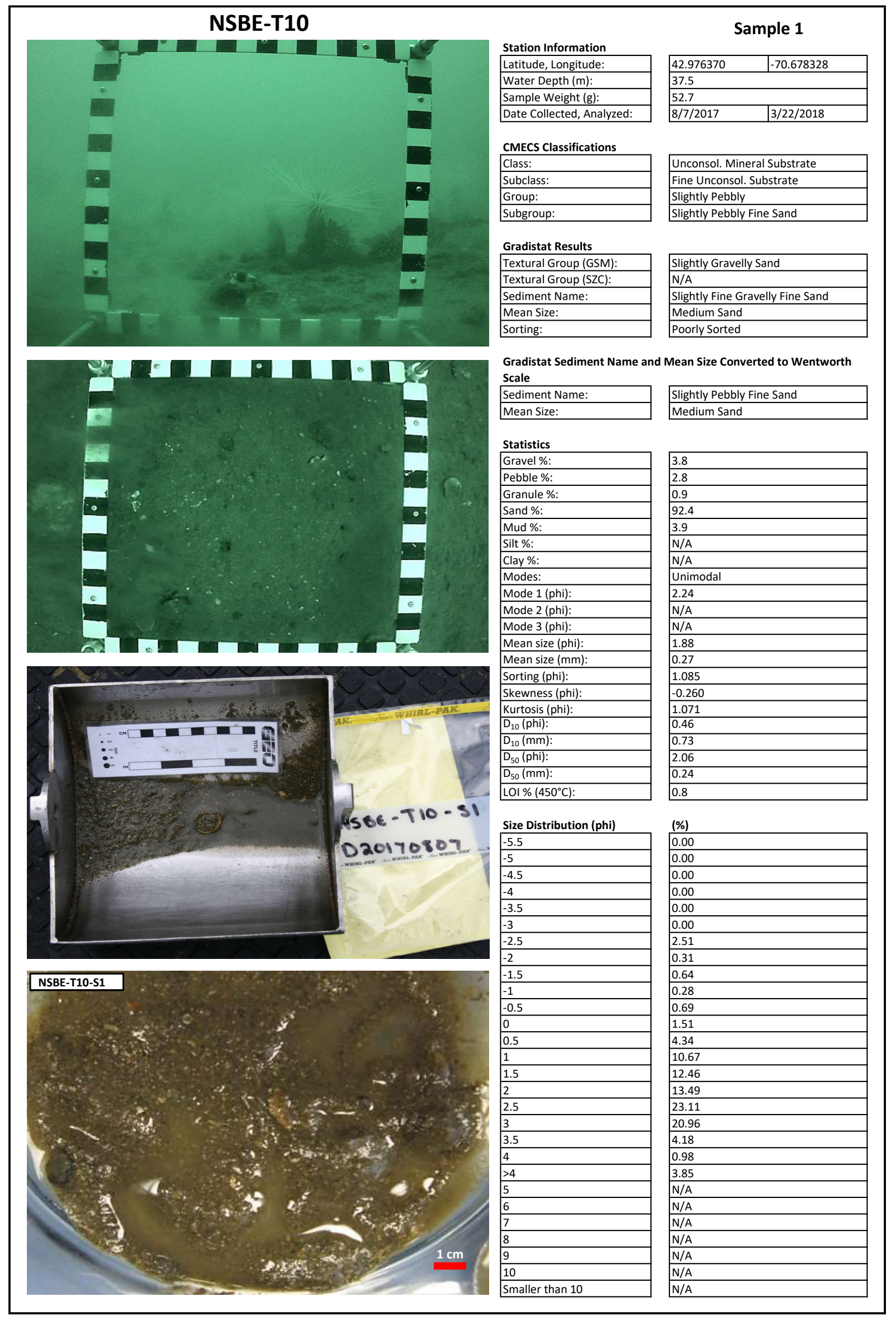




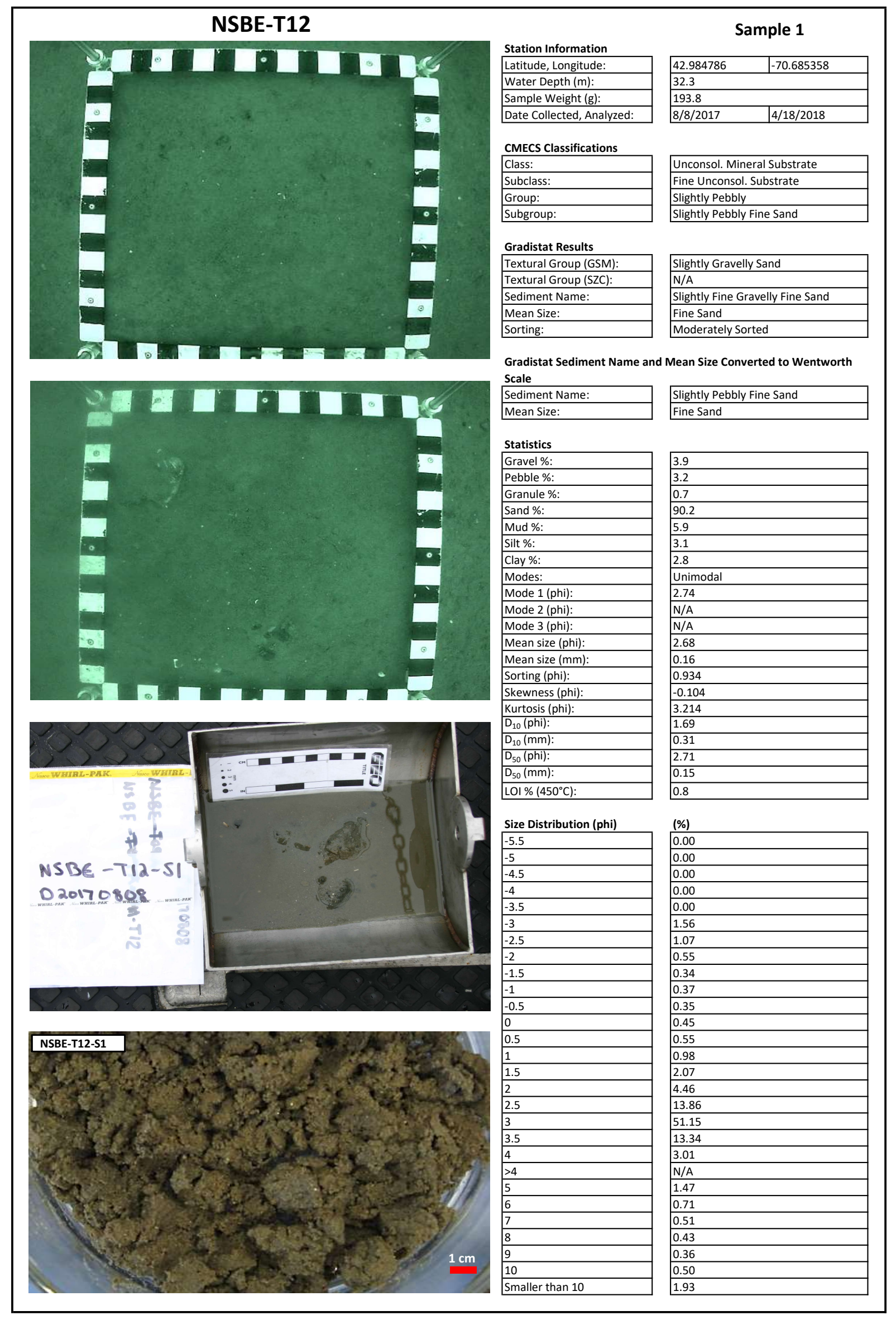




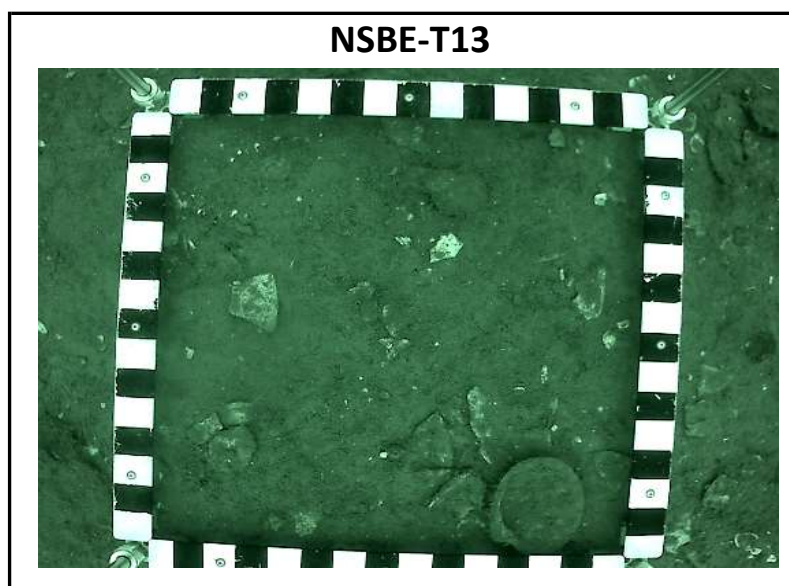

Station Information
\begin{tabular}{|l|}
\hline Latitude, Longitude: \\
\hline Water Depth (m): \\
\hline Sample Weight (g): \\
\hline Date Collected, Analyzed: \\
\hline
\end{tabular}

CMECS Classifications

\begin{tabular}{|l}
\hline Class: \\
\hline Subclass: \\
\hline
\end{tabular}

Group:

Subgroup:

Gradistat Results

Textural Group (GSM):

Textural Group (SZC):

Sediment Name:

Mean Size:

Sorting:

Gradistat Sediment Name and Mean Size Converted to Wentworth Scale
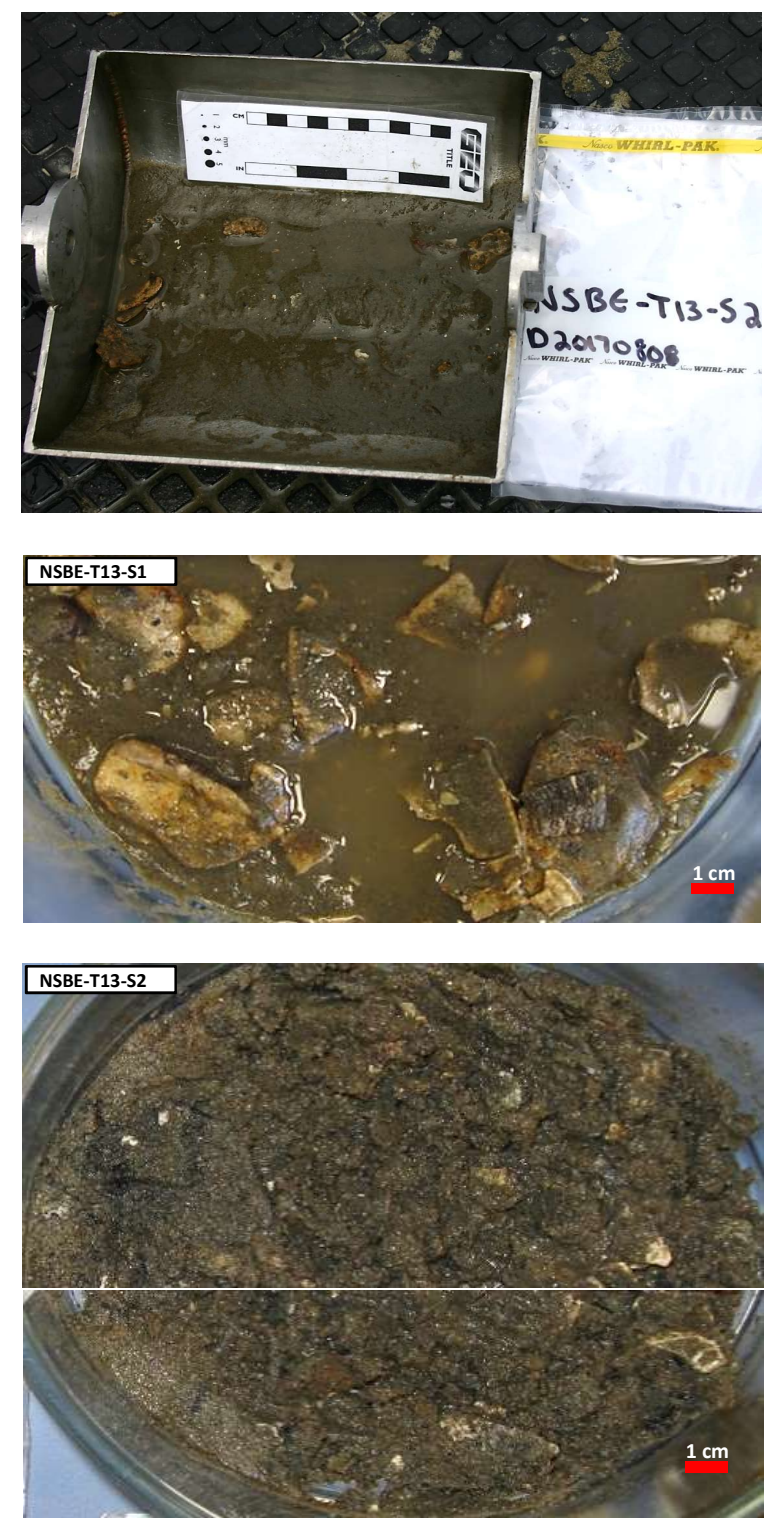

Sediment Name:

Mean Size:

Statistics

Gravel \%:

Pebble \%:

Granule \%:

Sand \%:

Mud \%:

Silt \%:

Clay \%:

Modes:

\begin{tabular}{l}
\hline Mode 1 (phi): \\
\hline Mode 2 (phi):
\end{tabular}

Mode 2 (phi):

Mode 3 (phi):

Mean size (phi):

Mean size $(\mathrm{mm})$

Sorting (phi):

Skewness (phi):

Kurtosis (phi):

$\mathrm{D}_{10}$ (phi):

$\mathrm{D}_{10}(\mathrm{~mm})$ :

$\mathrm{D}_{50}$ (phi):

$\mathrm{D}_{50}(\mathrm{~mm}):$

LOI \% $\left(450^{\circ} \mathrm{C}\right)$ :

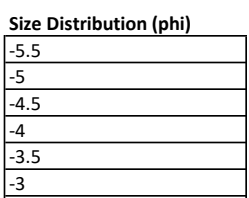

$-2.5$

\begin{tabular}{|l}
\hline-2.5 \\
\hline-2 \\
\hline-1.5 \\
\hline
\end{tabular}

$-1.5$

\begin{tabular}{|l|}
\hline-1 \\
\hline-0.5 \\
\hline
\end{tabular}

\begin{tabular}{|l|}
\hline-0.5 \\
\hline 0 \\
\hline 0.5 \\
\hline
\end{tabular}

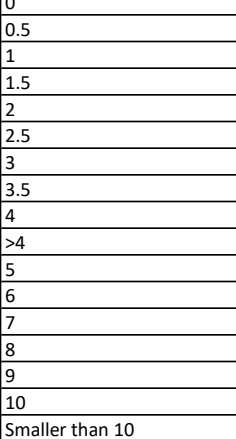

Slightly Pebbly Fine Sand
Sample 1

\begin{tabular}{|l|l|}
\hline 42.987658 & -70.660873 \\
\hline 30.5 & \\
\hline 141.8 & $3 / 29 / 2018$ \\
\hline $8 / 8 / 2017$ & \\
\hline
\end{tabular}

Sample 2

\begin{tabular}{|l|l|}
\hline 42.987847 & -70.660993 \\
\hline 30.5 & \\
\hline 157.1 & $2 / 21 / 2018$ \\
\hline $8 / 8 / 2017$ & \\
\hline
\end{tabular}

Unconsol. Mineral Substrate

Fine Unconsol. Substrate

Slightly Pebbly

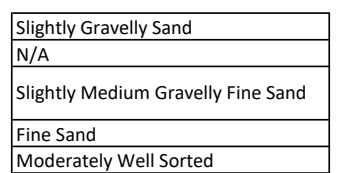

Moderately Well Sorted

Slightly Pebbly Fine Sand

Fine Sand

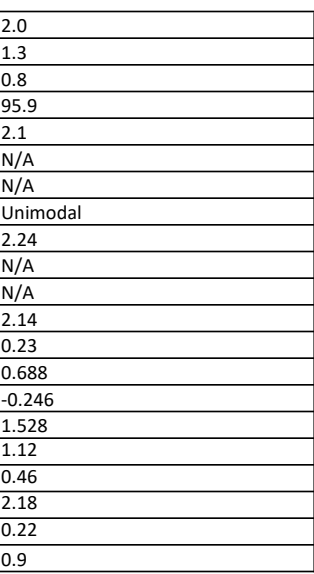

(\%)

\begin{tabular}{|l}
0.00 \\
\hline 0.00 \\
\hline
\end{tabular}

\begin{tabular}{l}
0.00 \\
\hline 0.00 \\
0.00 \\
\hline
\end{tabular}

\begin{tabular}{l}
0.00 \\
\hline 0.00 \\
\hline 0.00 \\
\hline
\end{tabular}

0.00

1.09

\begin{tabular}{l}
0.00 \\
\hline 0.17 \\
\hline 0.00 \\
0.19
\end{tabular}

\begin{tabular}{l}
0.00 \\
\hline 0.19 \\
0.57
\end{tabular}

\begin{tabular}{l}
0.17 \\
\hline 0.19 \\
\hline 0.57 \\
\hline 0.53 \\
\hline
\end{tabular}

\begin{tabular}{l}
0.57 \\
\hline 0.53 \\
\hline
\end{tabular}

\begin{tabular}{l}
0.53 \\
\hline 1.05 \\
\hline 1.82
\end{tabular}

1.82

\begin{tabular}{|l}
1.82 \\
\hline 3.12 \\
\hline 5.92 \\
\hline 19.46
\end{tabular}

\begin{tabular}{|l|l|}
\hline 3.12 \\
\hline 19.46 \\
\hline 41.52 \\
\hline
\end{tabular}

\begin{tabular}{ll}
\hline 19.46 \\
\hline 41.52 \\
\hline 19.76 \\
\hline
\end{tabular}

\begin{tabular}{|l}
\hline 19.52 \\
\hline 19.76 \\
\hline 2.42 \\
\hline
\end{tabular}

2.42

\begin{tabular}{|l|}
\hline 2.42 \\
\hline 0.31 \\
\hline 2.07 \\
\hline$N / A$
\end{tabular}

\begin{tabular}{|l|}
\hline 2.32 \\
\hline N/A \\
\hline N/A
\end{tabular}

N/A

N/A

\begin{tabular}{ll}
$\mathrm{N} / \mathrm{A}$ \\
\hline $\mathrm{N} / \mathrm{A}$ \\
\hline $\mathrm{N} / \mathrm{A}$
\end{tabular}

\begin{tabular}{ll}
\hline$N / A$ \\
\hline$N / A$ \\
\hline
\end{tabular}

$\mathrm{N} / \mathrm{A}$

$\mathrm{N} / \mathrm{A}$ \begin{tabular}{|l|}
\hline Slightly Pebbly Fine Sand \\
\hline Fine Sand \\
\hline
\end{tabular}

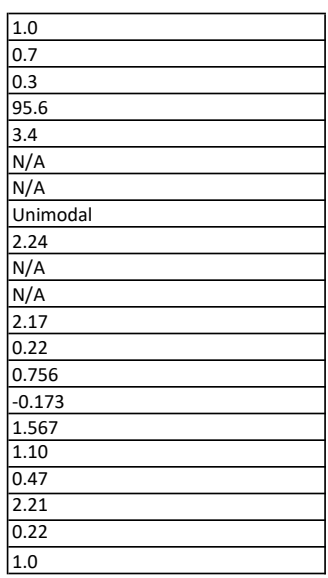

Unconsol. Mineral Substrate

Fine Unconsol. Substrate

Slightly Pebbly

Slightly Pebbly Fine Sand

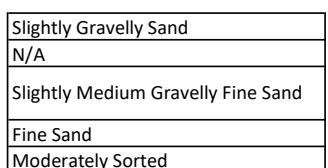

Moderately Sorted

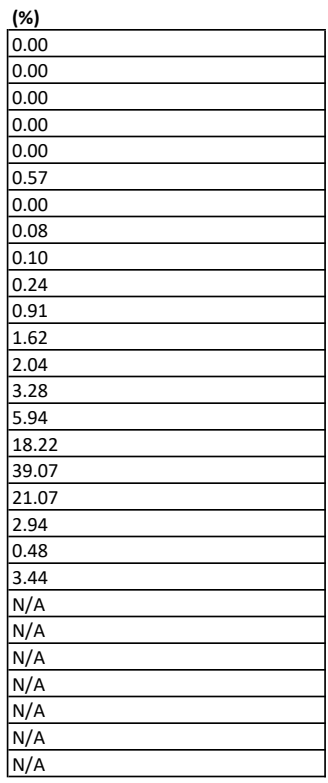




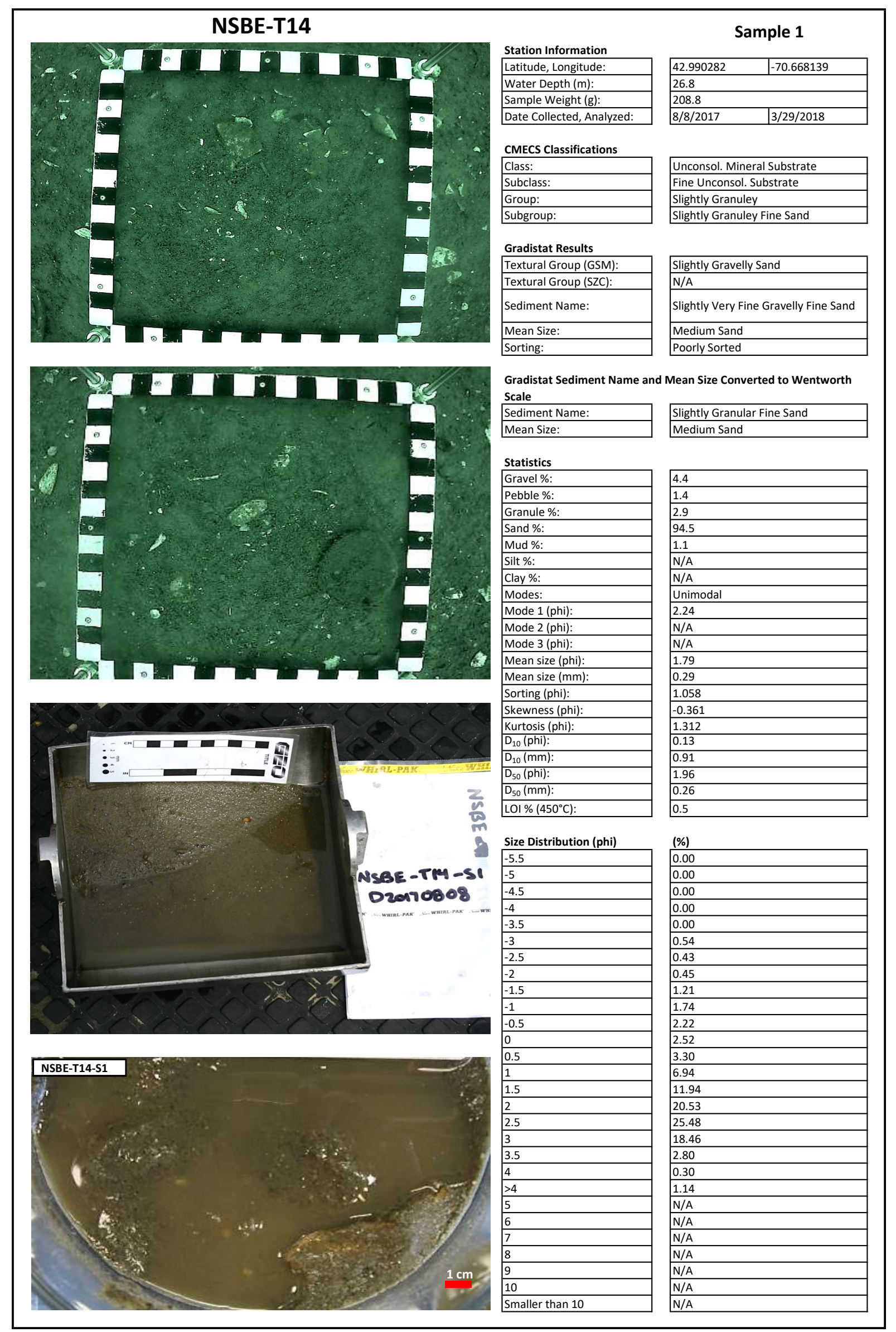




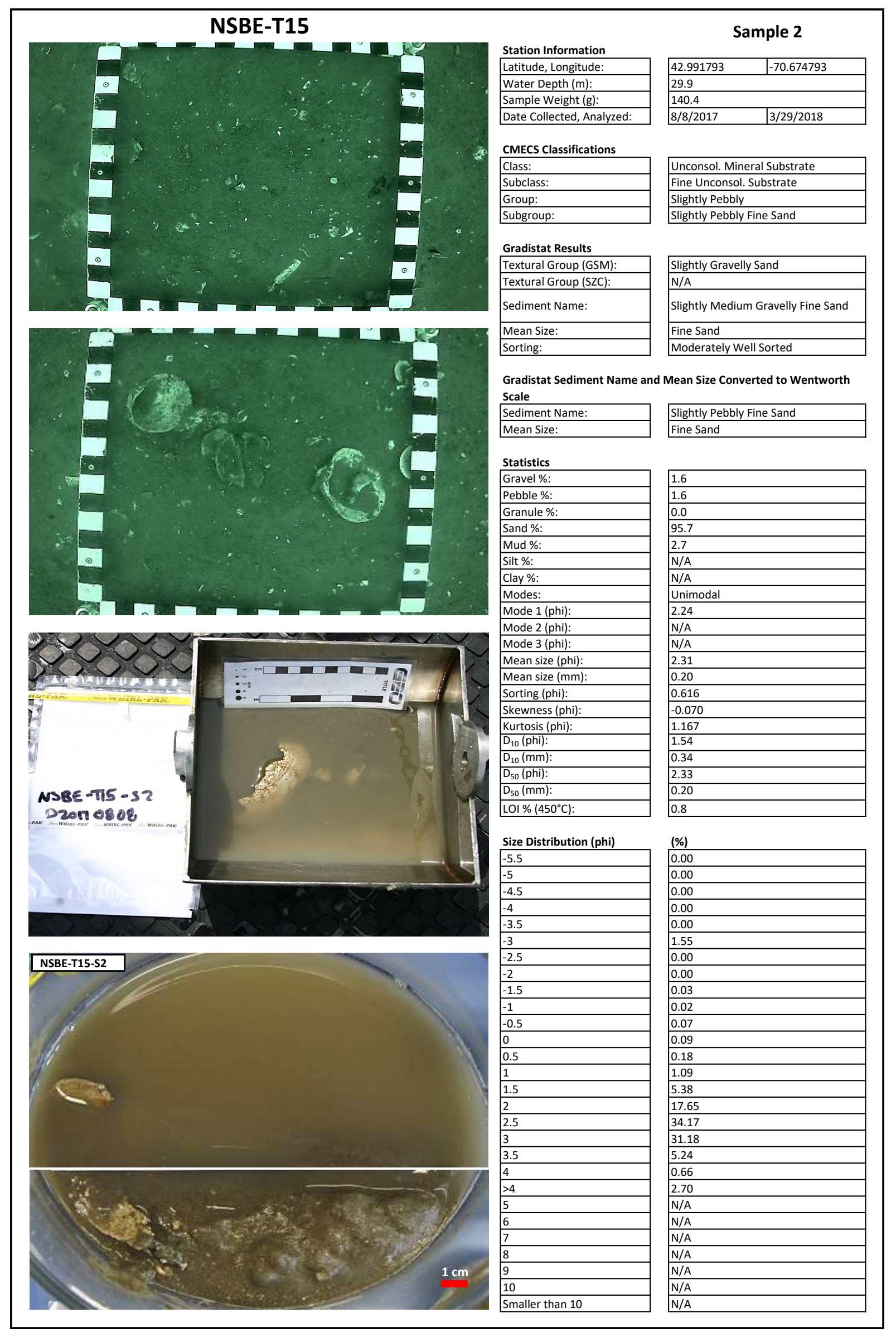




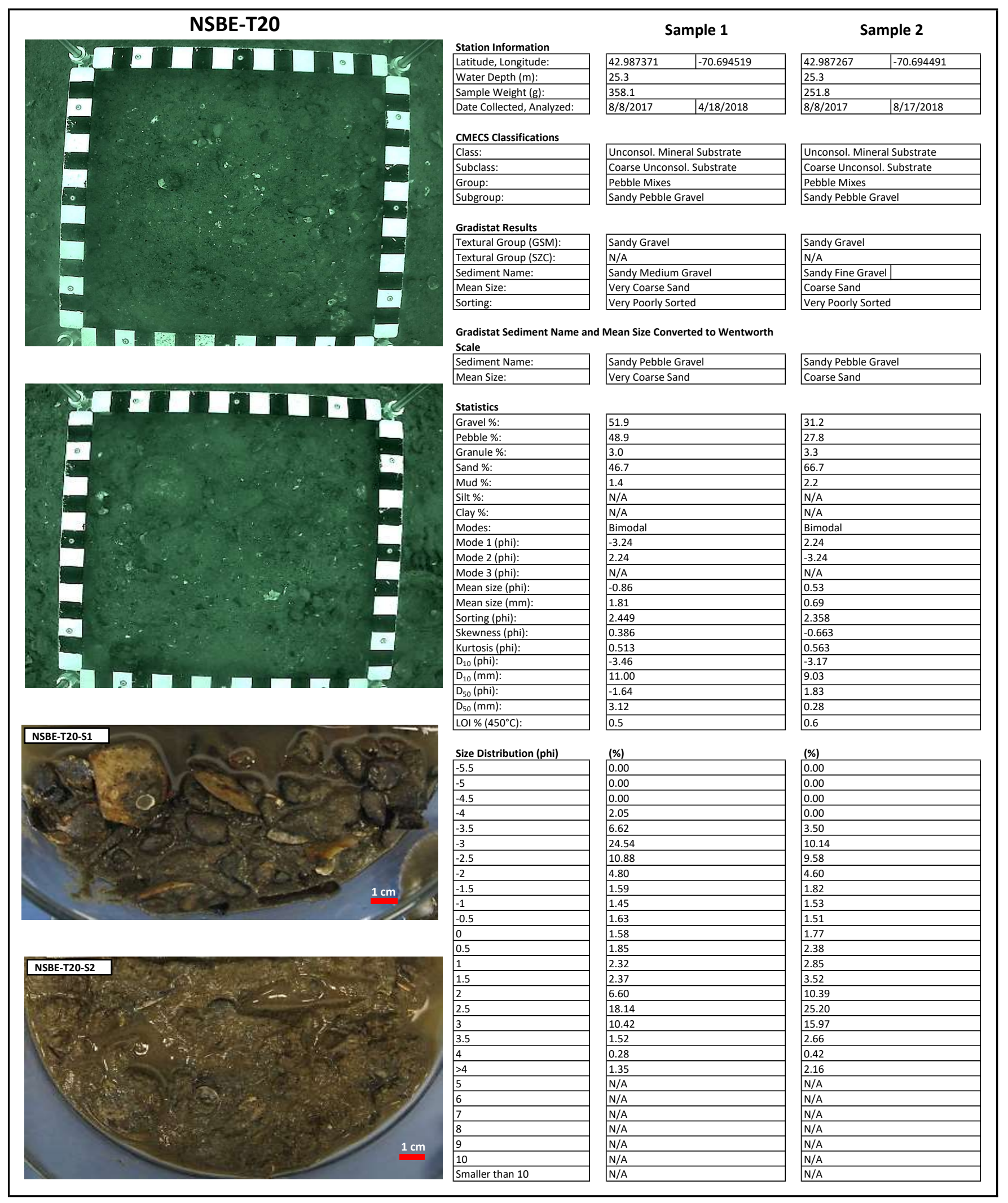




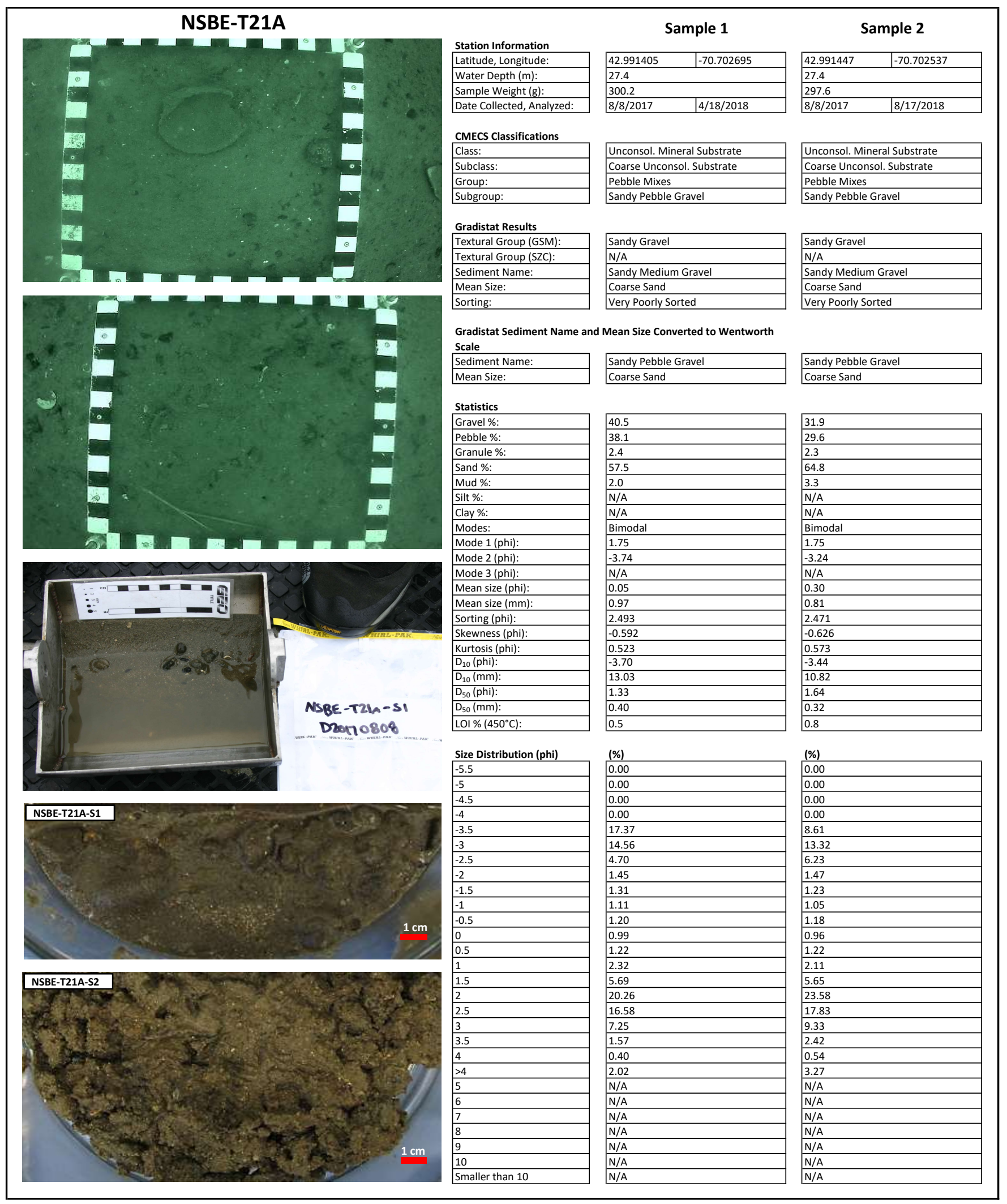




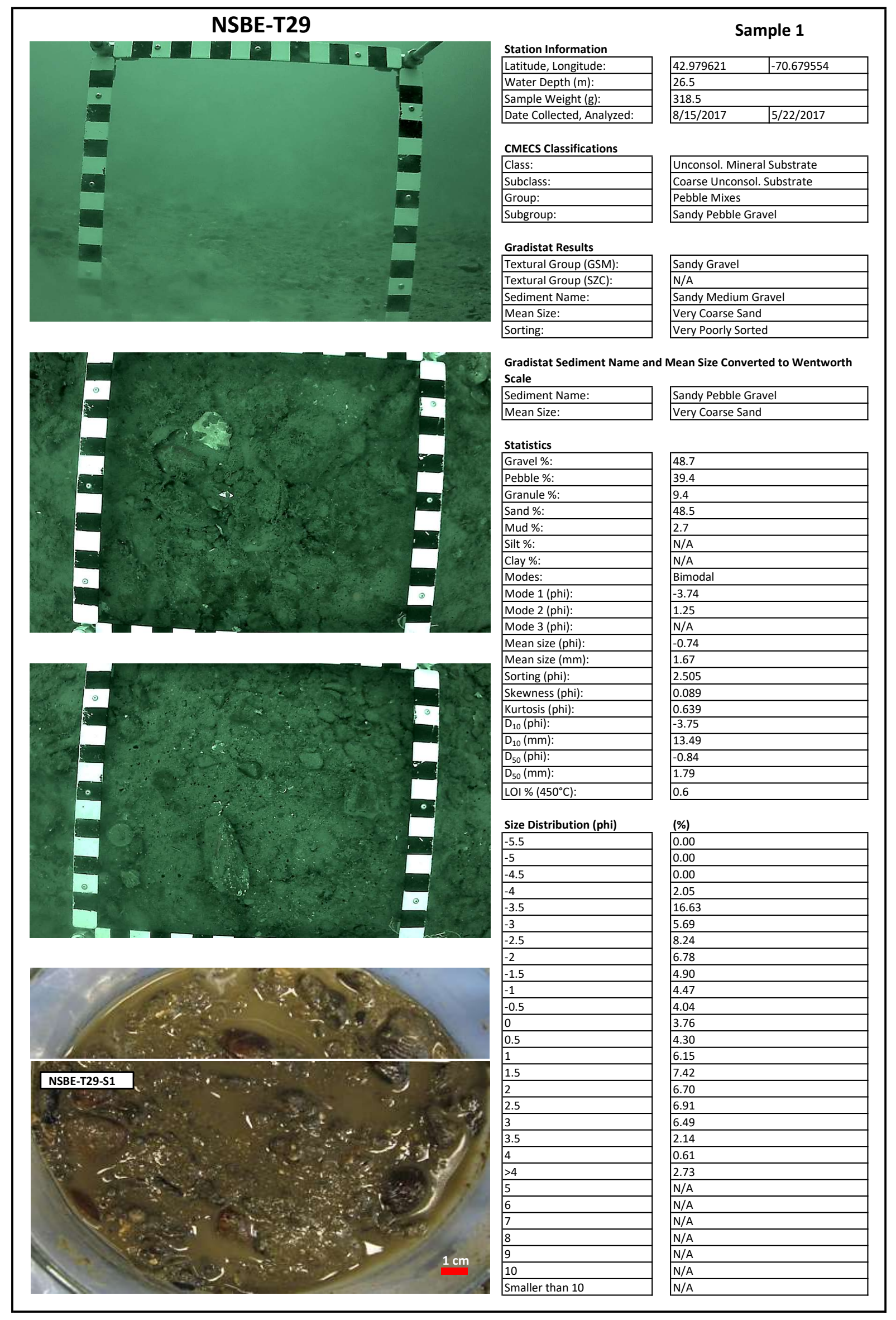




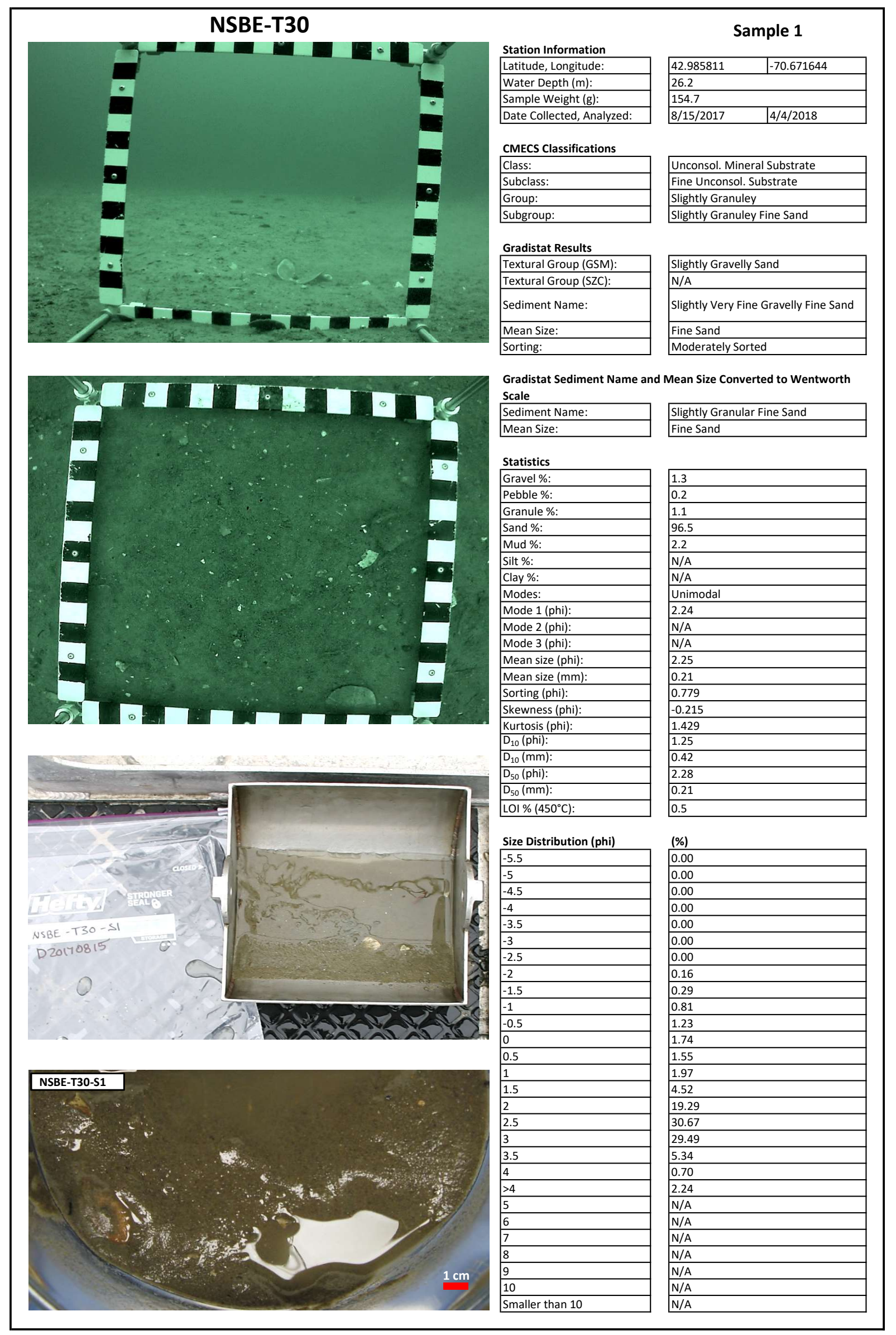




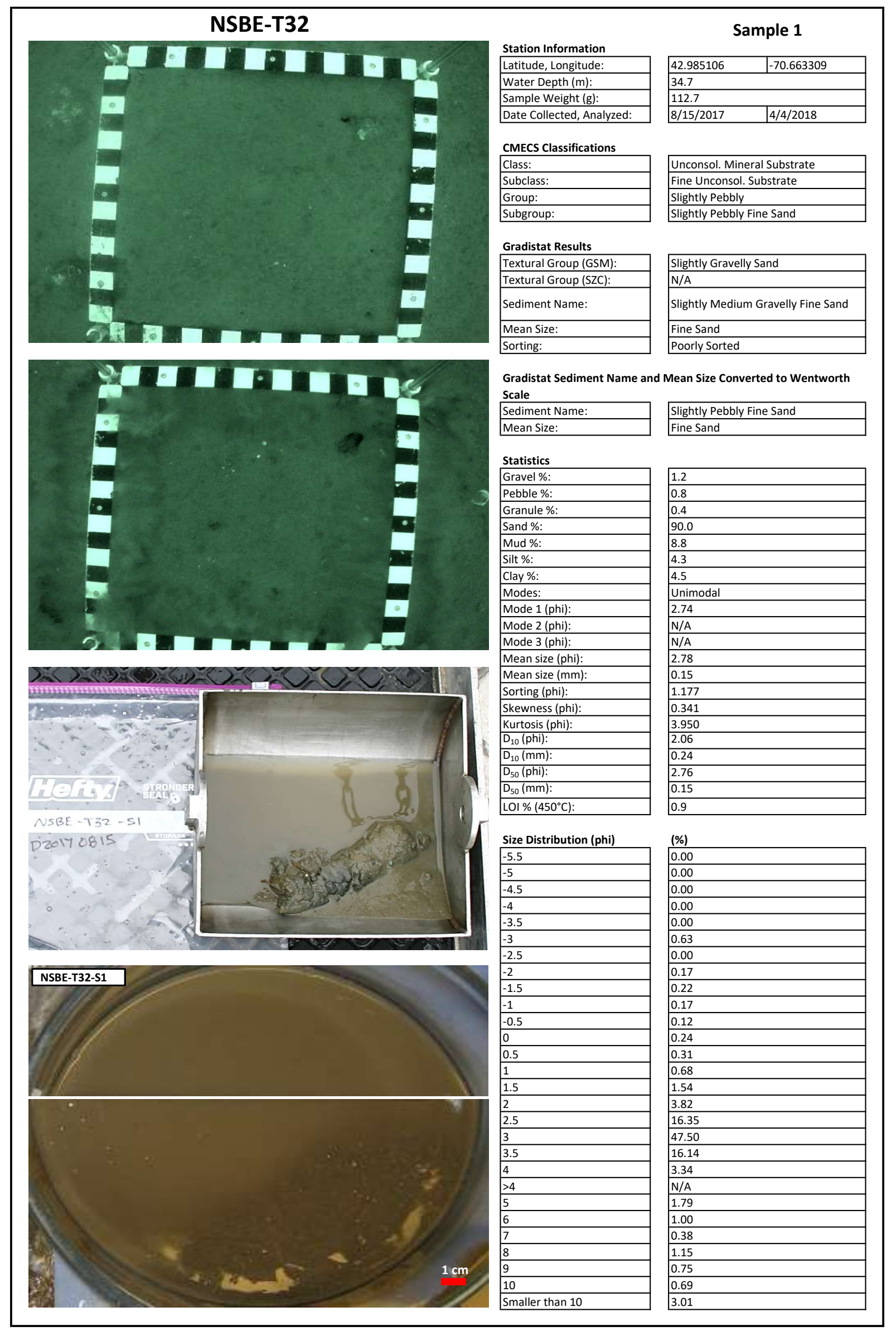




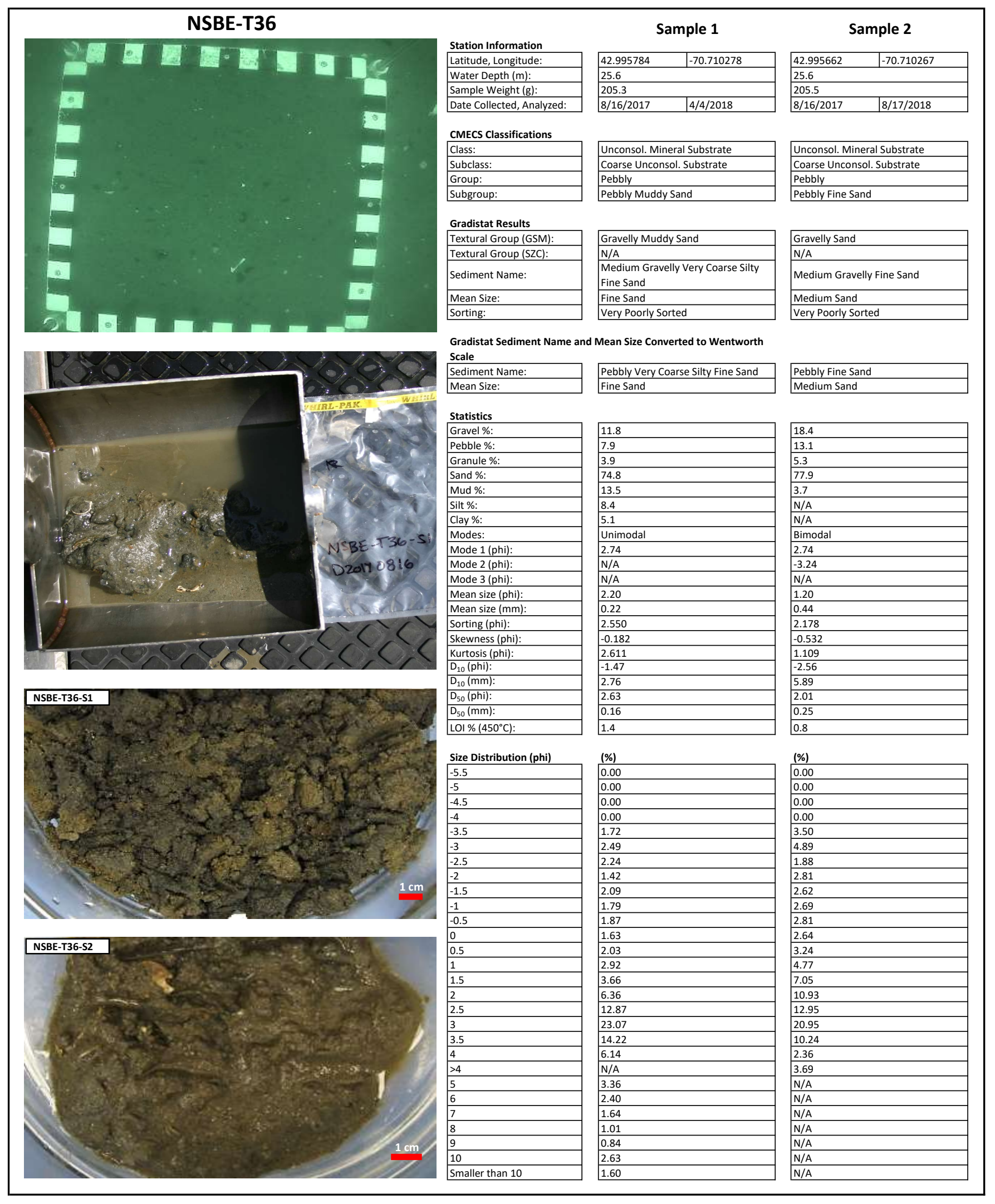




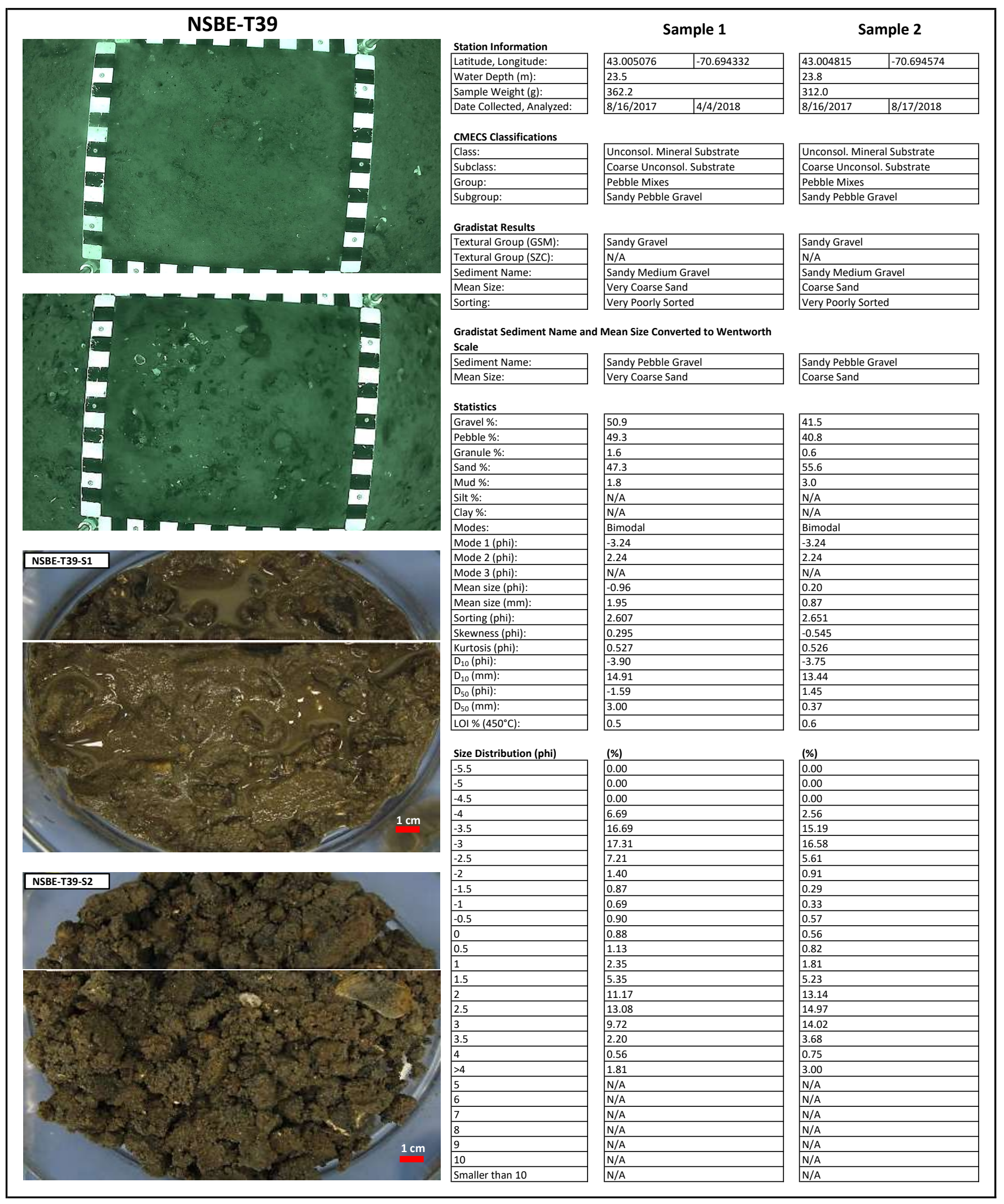




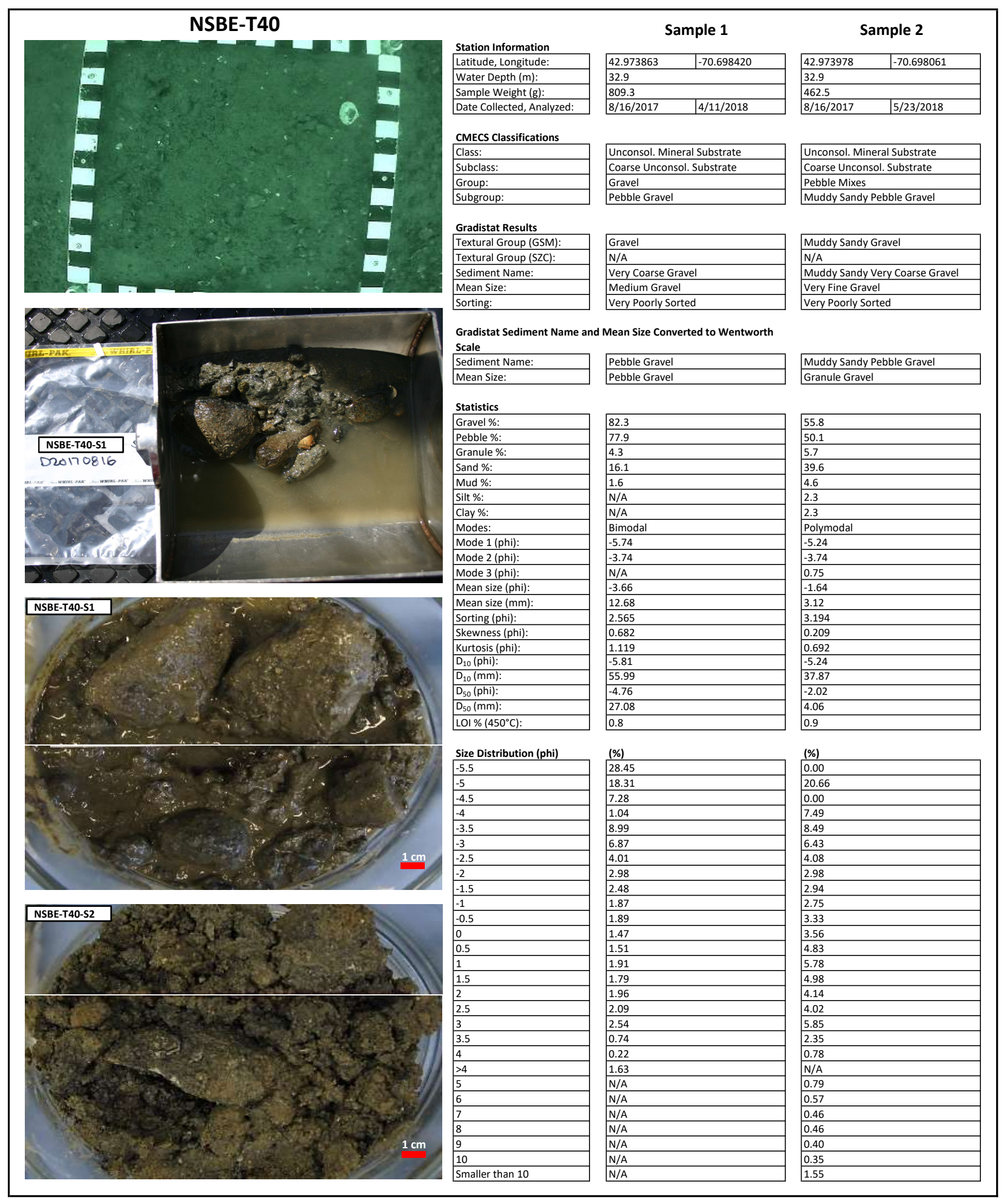




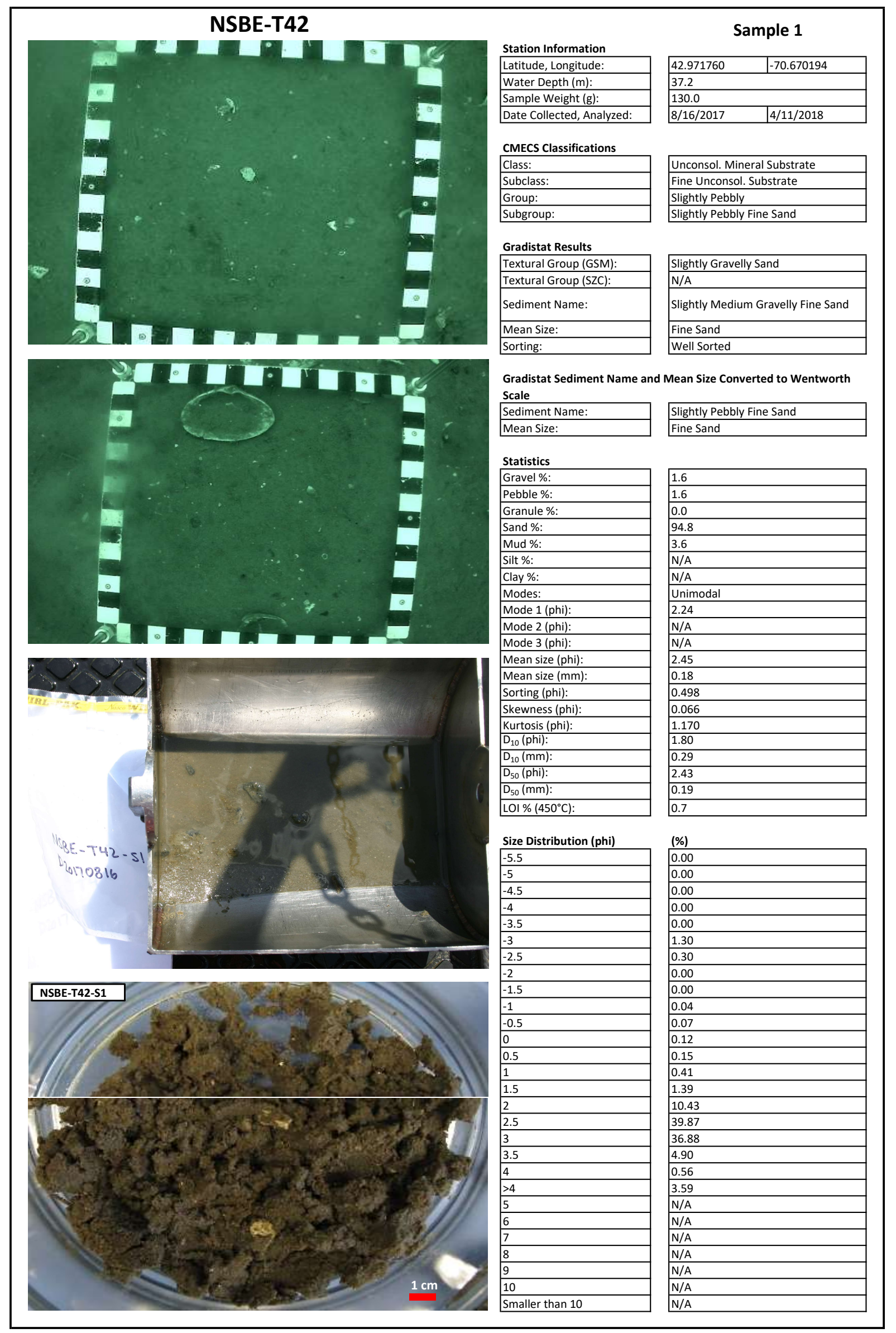




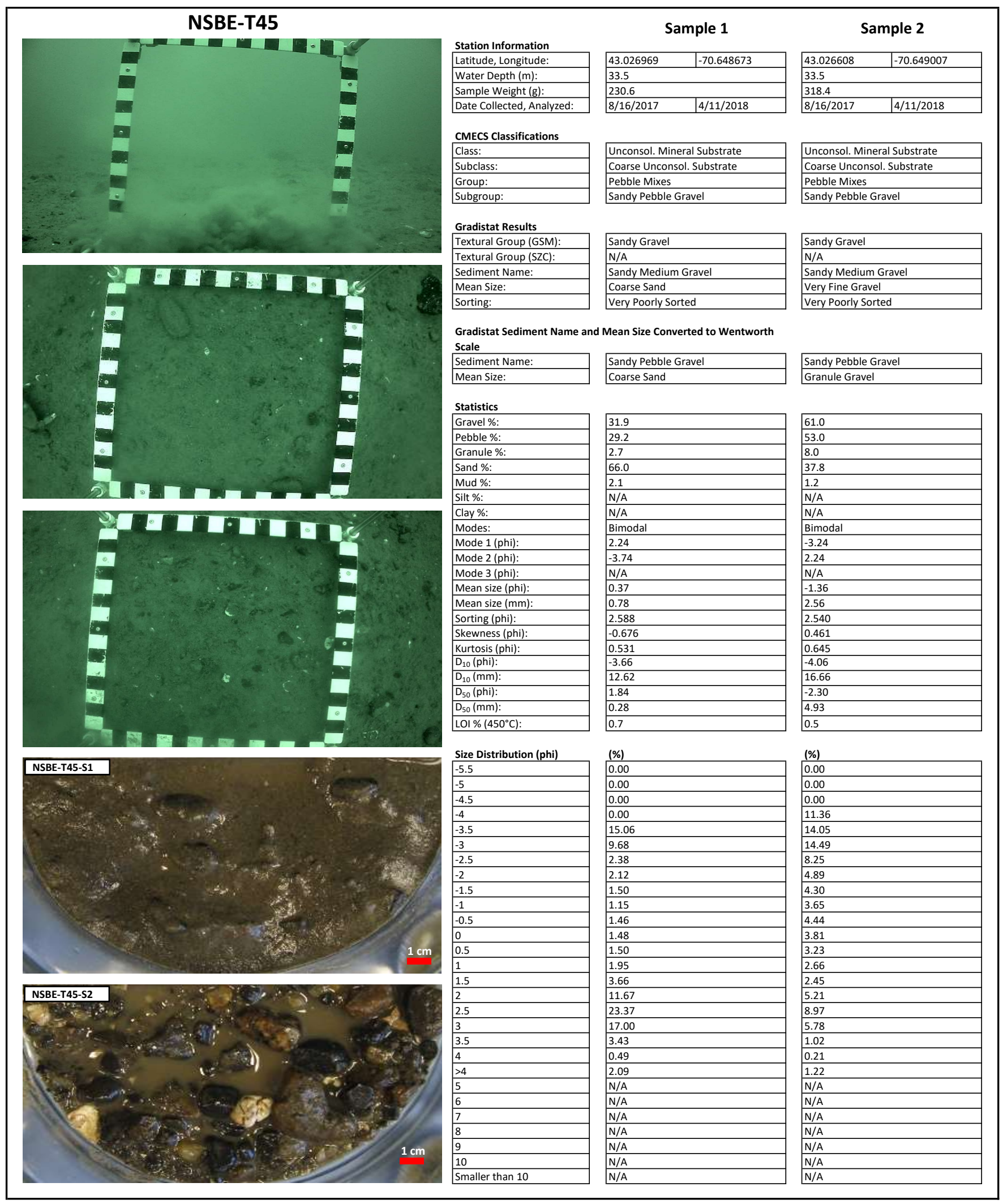




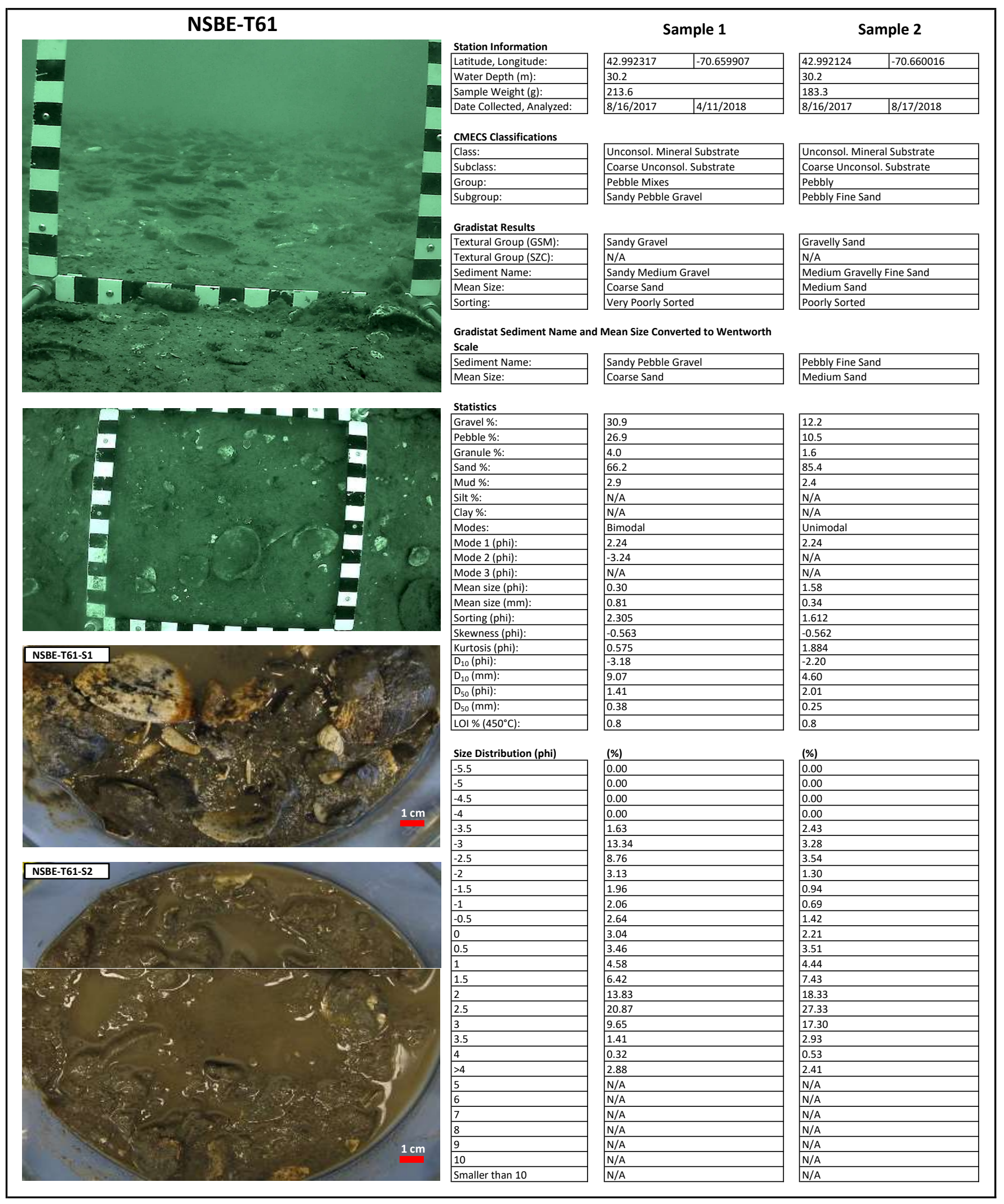




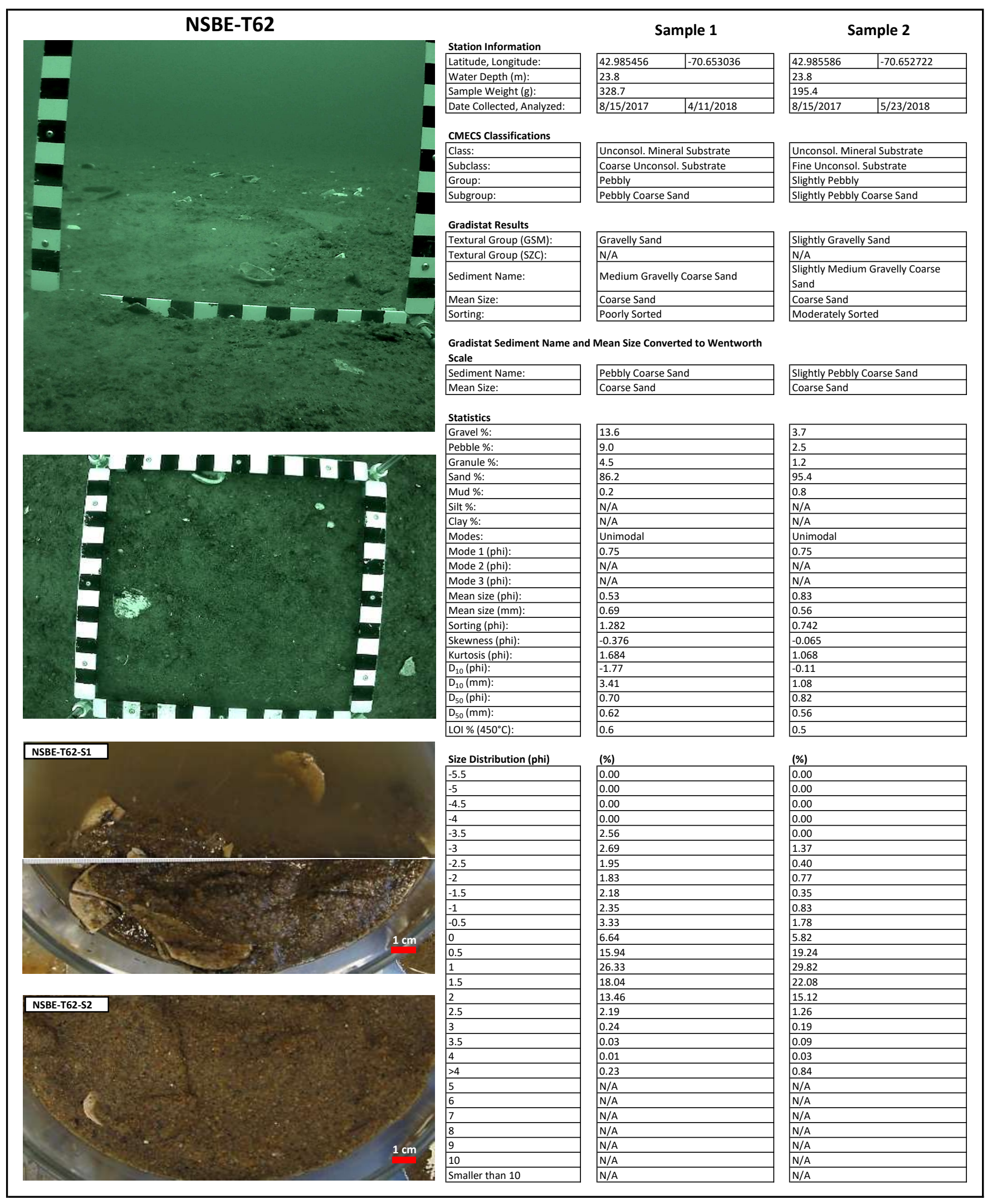




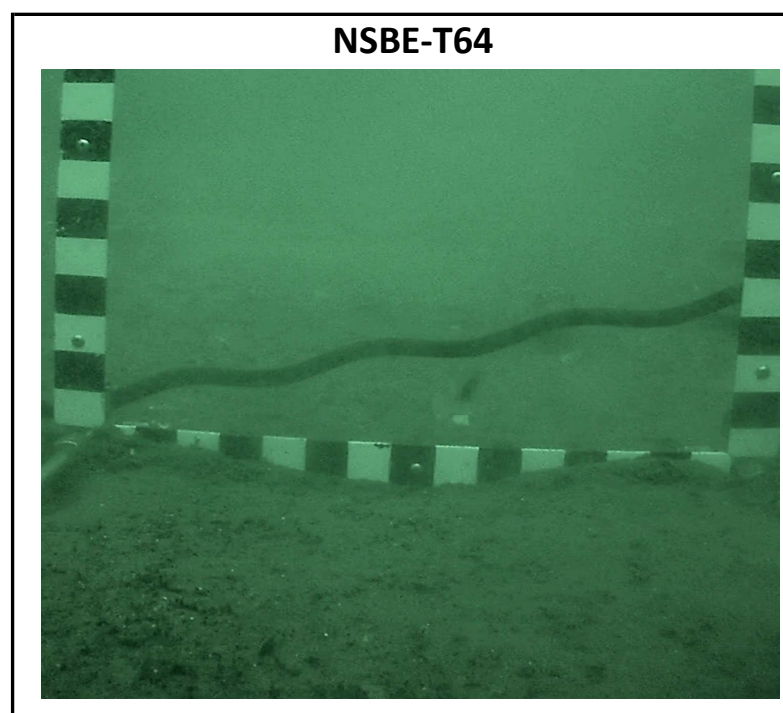

Station Information
\begin{tabular}{|l|}
\hline Latitude, Longitude: \\
\hline Water Depth (m): \\
\hline Sample Weight (g): \\
\hline Date Collected, Analyzed: \\
\hline
\end{tabular}

CMECS Classifications

\begin{tabular}{l} 
Class: \\
\hline Subclass: \\
\hline
\end{tabular}

Group:

Subgroup:

Gradistat Results

Textural Group (GSM):

Textural Group (SZC):

Sediment Name:

Mean Size:

Sorting:

Gradistat Sediment Name and Mean Size Converted to Wentworth

Scale

Sediment Name:

Mean Size:
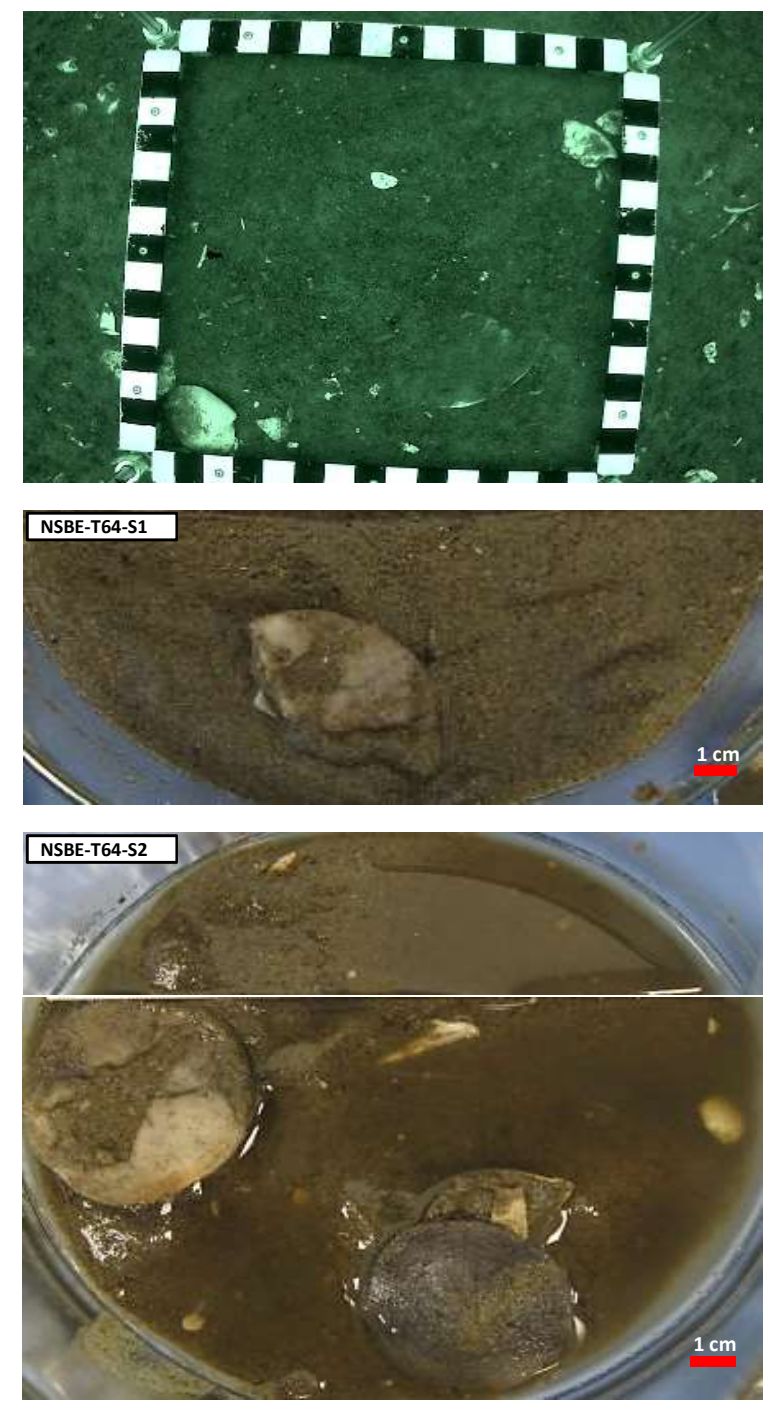

Statistics

Gravel \%:

Pebble \%:

Granule \%:

Sand \%:

Mud \%:

Silt \%:

Clay \%:

Modes:

Mode 1 (phi):

Mode 2 (phi):

Mode 3 (phi):

Mean size (phi):

Mean size $(\mathrm{mm})$

Sorting (phi):

Skewness (phi):

Kurtosis (phi):

$\mathrm{D}_{10}$ (phi)

$\mathrm{D}_{10}(\mathrm{~mm})$ :

$\mathrm{D}_{50}$ (phi):

$\mathrm{D}_{50}(\mathrm{~mm}):$

LOI $\%\left(450^{\circ} \mathrm{C}\right)$ :

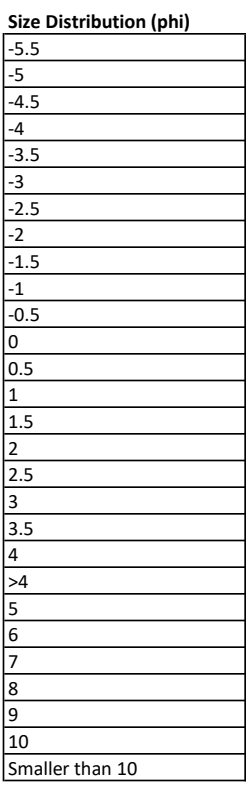

Sample 1

\begin{tabular}{|l|l|}
\hline 42.978964 & -70.663495 \\
\hline 25.6 & \\
\hline 182.2 & $4 / 18 / 2018$ \\
\hline $8 / 15 / 2017$ & \\
\hline
\end{tabular}

Sample 2

\begin{tabular}{|l|l|}
\hline 42.979064 & -70.663322 \\
\hline 25.6 & \\
\hline 112.7 & $5 / 23 / 2018$ \\
\hline $8 / 15 / 2017$ & \\
\hline
\end{tabular}

Unconsol. Mineral Substrate

Fine Unconsol. Substrate

Slightly Granuley

Slightly Granuley Medium Sand

Unconsol. Mineral Substrate

Fine Unconsol. Substrate

Slightly Granuley

Slightly Granuley Medium Sand

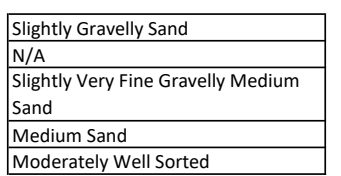

Slightly Gravelly Sand

\begin{tabular}{l} 
N/A \\
\hline Slightly Very Fine Gravelly Medium
\end{tabular} Sand

Medium Sand

Moderately Well Sorted

Slightly Granular Medium Sand

Medium Sand

Slightly Granular Medium Sand

Medium Sand
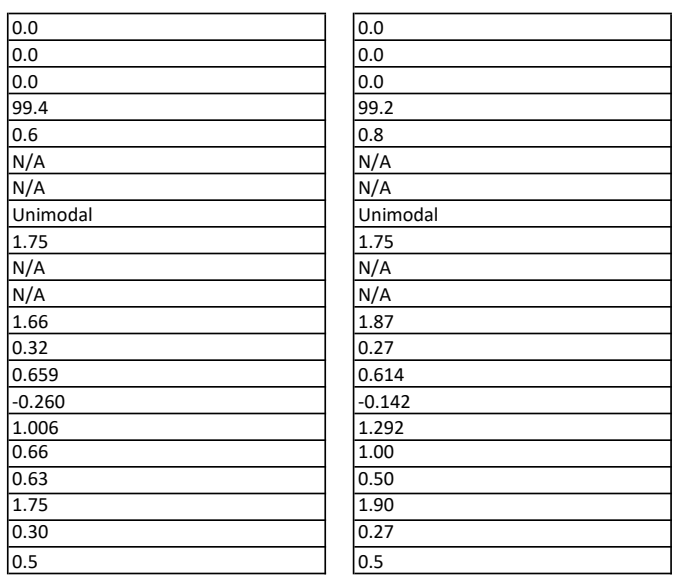

(\%)

0.00

\begin{tabular}{l}
0.00 \\
\hline 0.00 \\
\hline 0.00
\end{tabular}

\begin{tabular}{l}
0.00 \\
\hline 0.00 \\
\hline
\end{tabular}

\begin{tabular}{l}
0.00 \\
\hline 0.00 \\
\hline
\end{tabular}

\begin{tabular}{l}
0.00 \\
\hline 0.00
\end{tabular}

\begin{tabular}{l}
0.00 \\
\hline 0.00 \\
\hline 0.00
\end{tabular}

0.00

0.00

\begin{tabular}{l}
0.05 \\
\hline 0.19 \\
\hline 0.95
\end{tabular}

\begin{tabular}{l}
0.19 \\
\hline 0.95 \\
\hline 5.08 \\
\hline
\end{tabular}

5.08

11.26

13.92

\begin{tabular}{|l|}
\hline 36.19 \\
\hline 27.24 \\
\hline
\end{tabular}

\begin{tabular}{l}
27.24 \\
\hline 4.32 \\
\hline
\end{tabular}

4.32

\begin{tabular}{|l|}
\hline 0.19 \\
\hline 0.03 \\
\hline
\end{tabular}

\begin{tabular}{l}
\hline 0.03 \\
\hline 0.58 \\
\hline$N / A$
\end{tabular}

\begin{tabular}{|l|}
\hline 0.58 \\
\hline N/A \\
\hline
\end{tabular}

\begin{tabular}{|l}
\hline N/A \\
\hline N/A \\
\hline$N / A$ \\
\hline
\end{tabular}

\begin{tabular}{l}
$N / A$ \\
\hline$N / A$ \\
\hline$N / A$
\end{tabular}

\begin{tabular}{l}
$N / A$ \\
\hline$N / A$ \\
\hline$N / A$
\end{tabular}

N/A

N/A

\begin{tabular}{|l|}
\hline (\%) \\
\hline 0.00 \\
\hline 0.00 \\
\hline 0.00 \\
\hline 0.00 \\
\hline 0.00 \\
\hline 0.00 \\
\hline 0.00 \\
\hline 0.00 \\
\hline 0.00 \\
\hline 0.04 \\
\hline 0.16 \\
\hline 0.71 \\
\hline 3.04 \\
\hline 5.99 \\
\hline 10.13 \\
\hline 37.10 \\
\hline 31.65 \\
\hline 9.74 \\
\hline 0.60 \\
\hline 0.05 \\
\hline 0.79 \\
\hline N/A \\
\hline N/A \\
\hline N/A \\
\hline N/A \\
\hline N/A \\
\hline N/A \\
\hline N/A \\
\hline
\end{tabular}




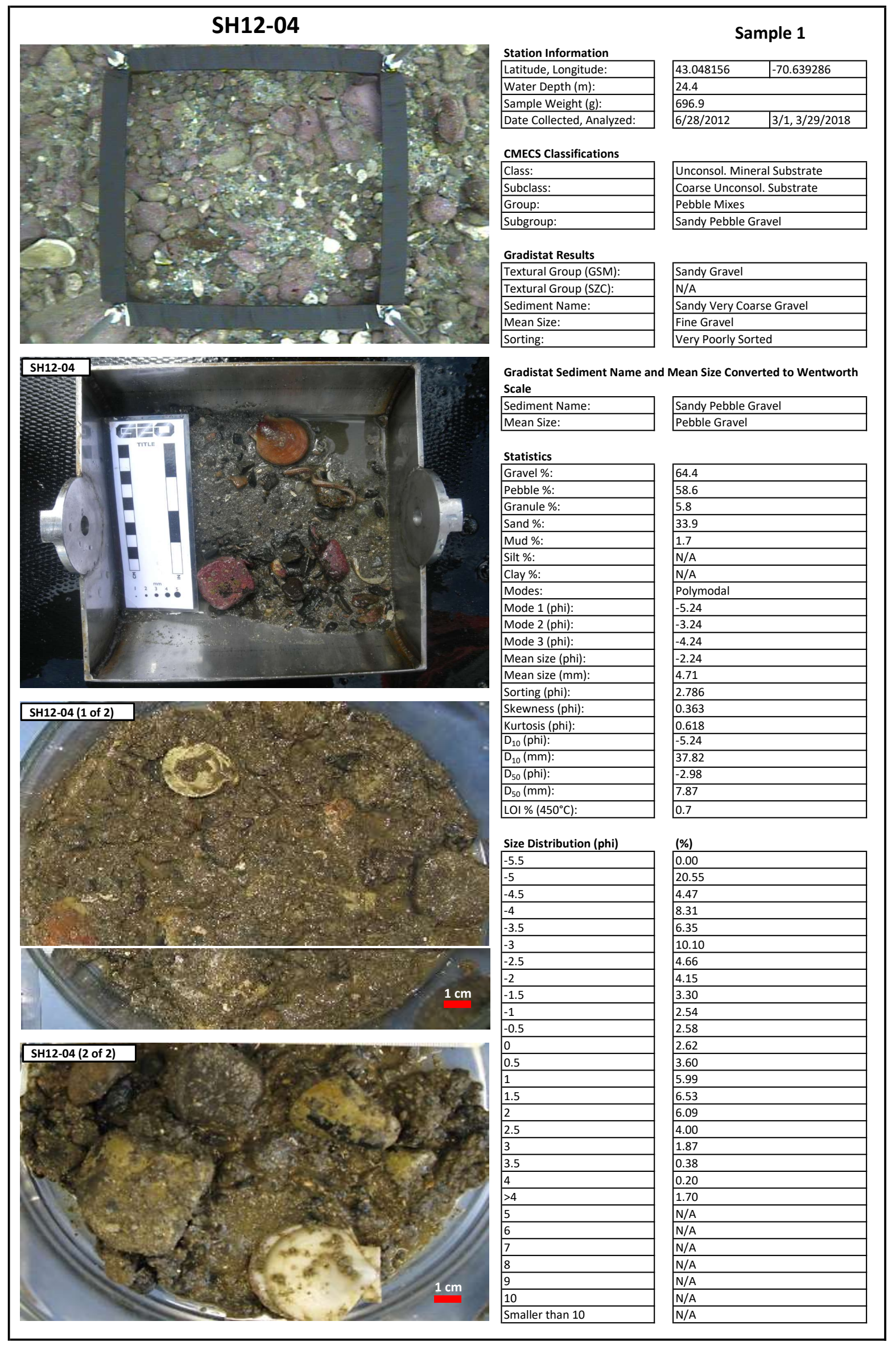




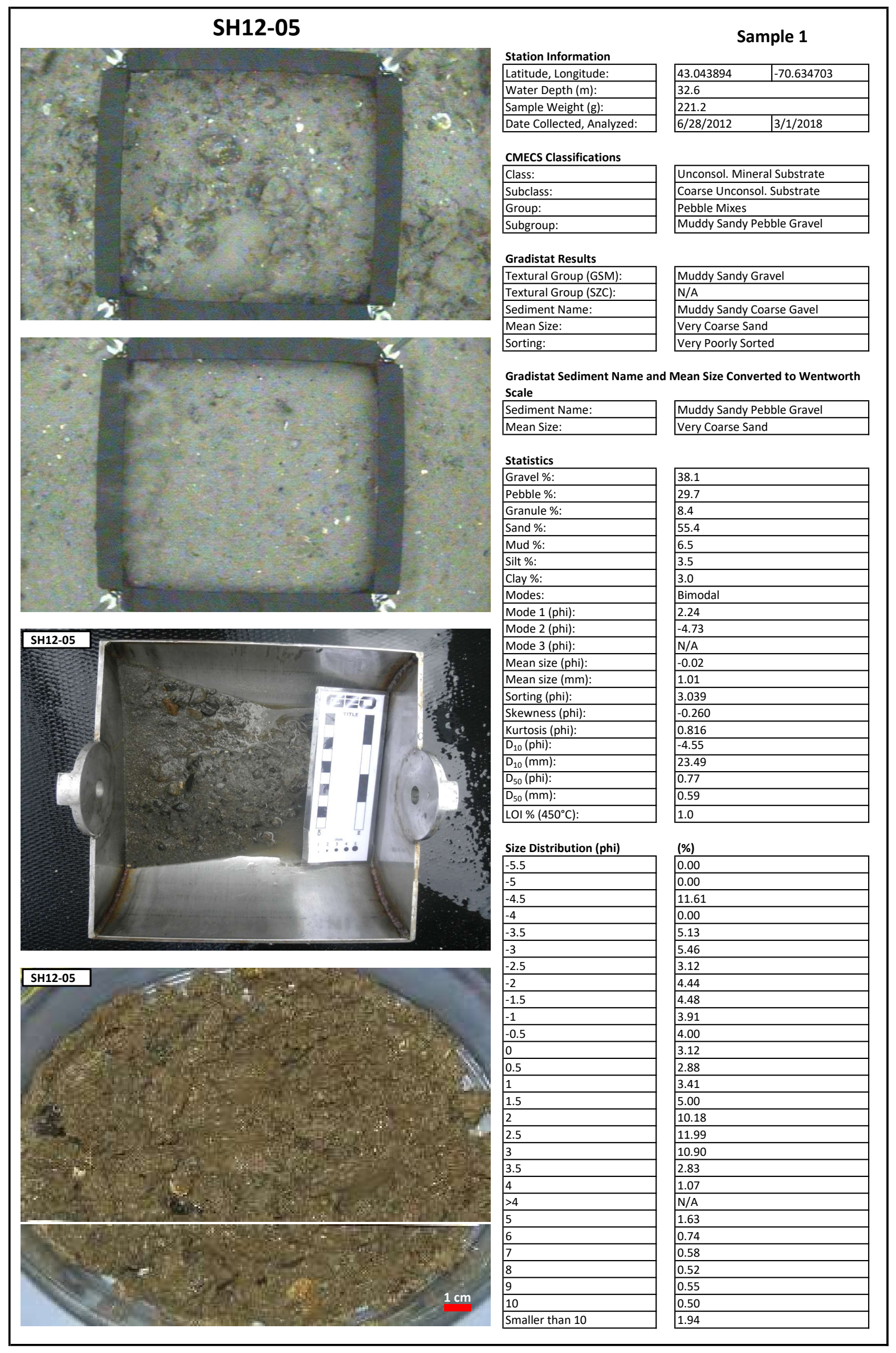




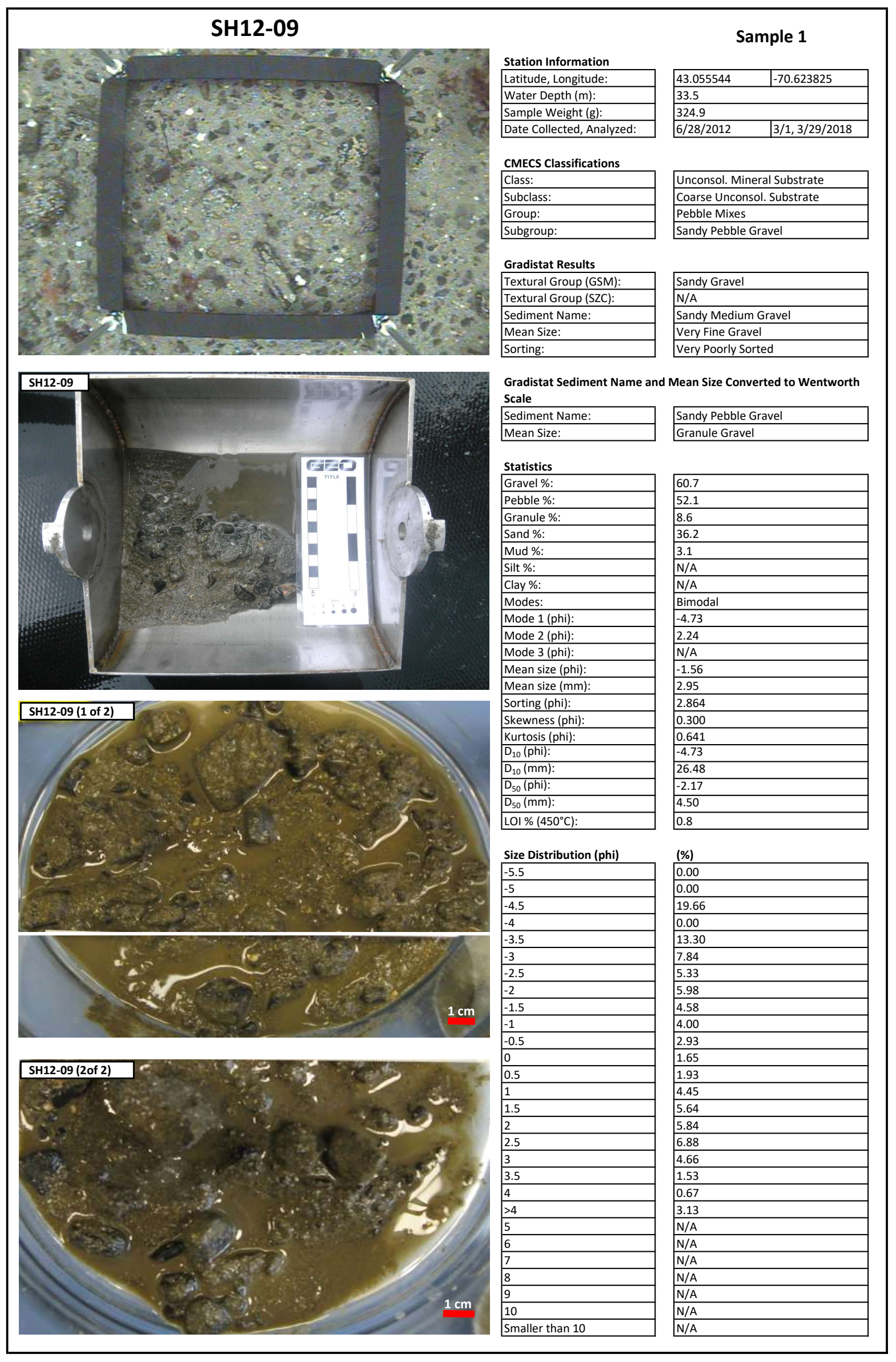




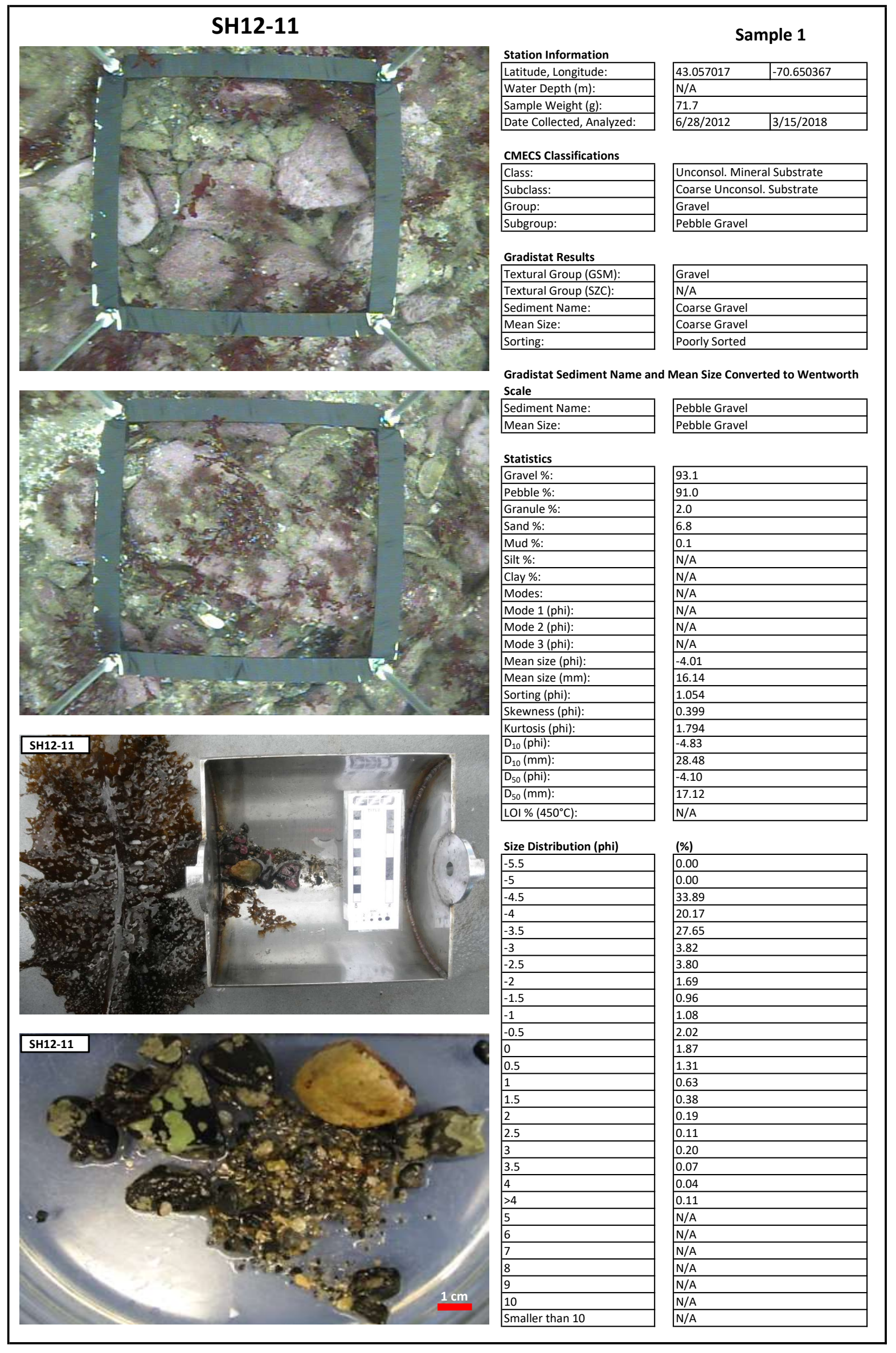




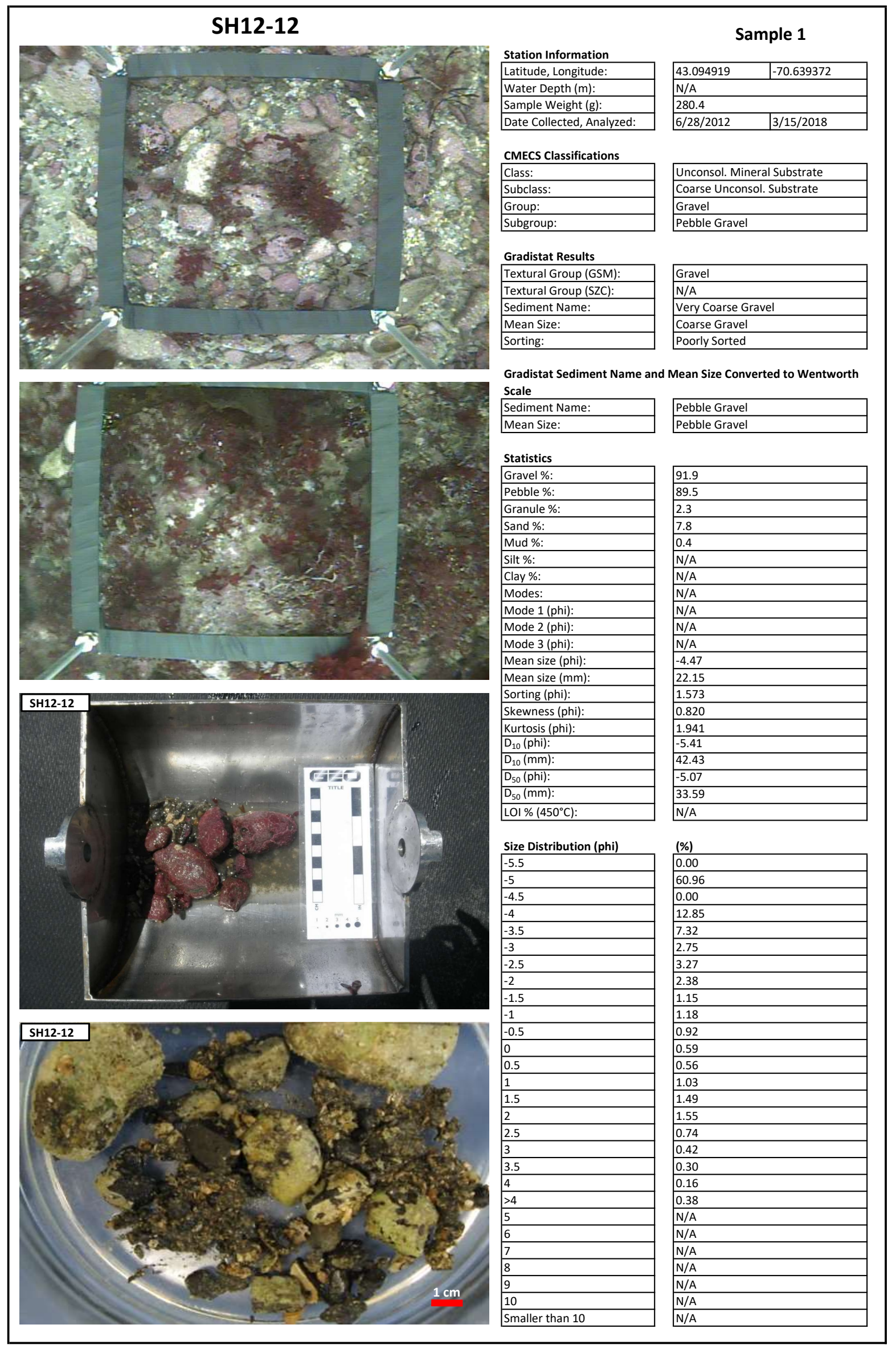




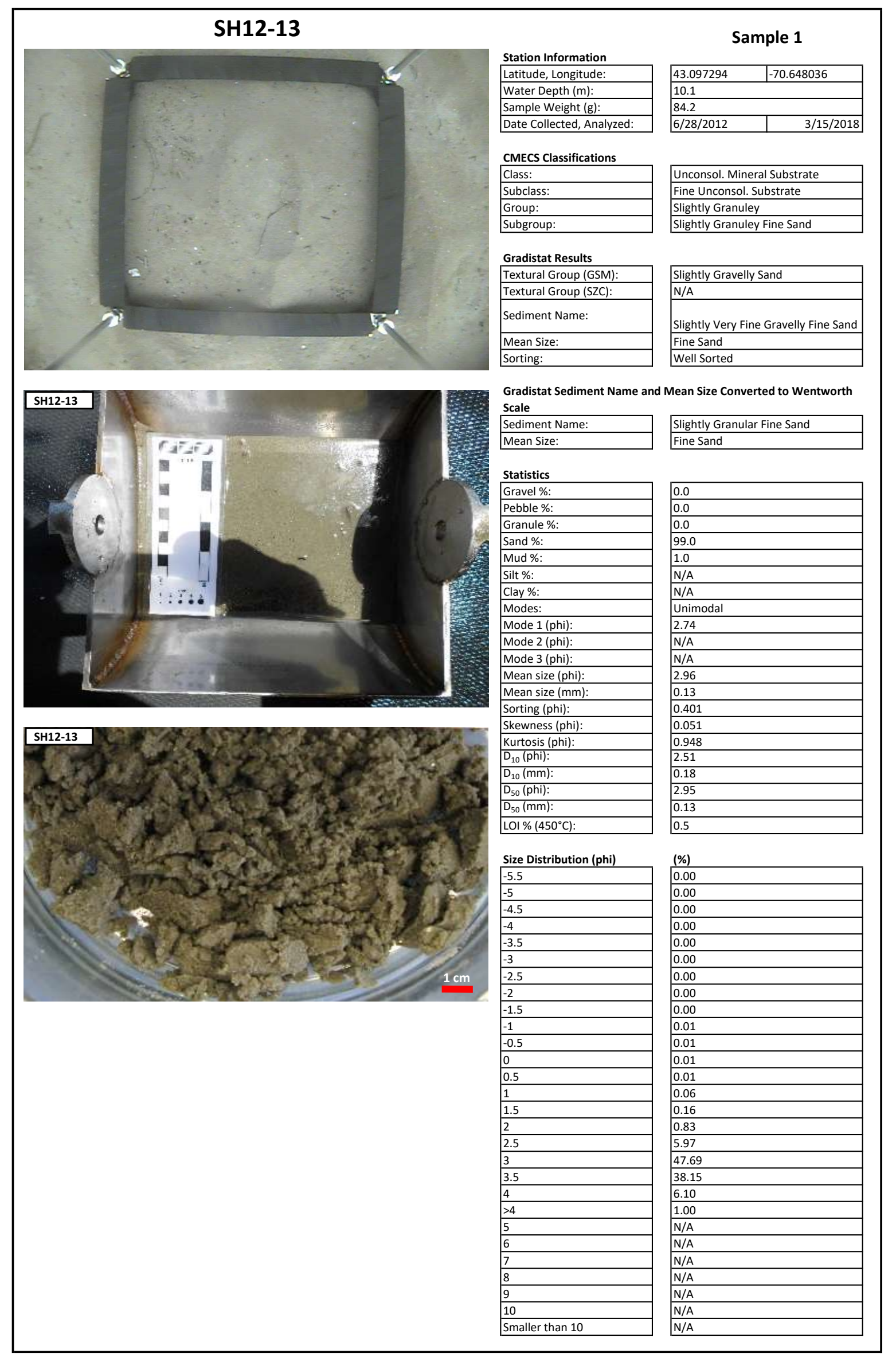




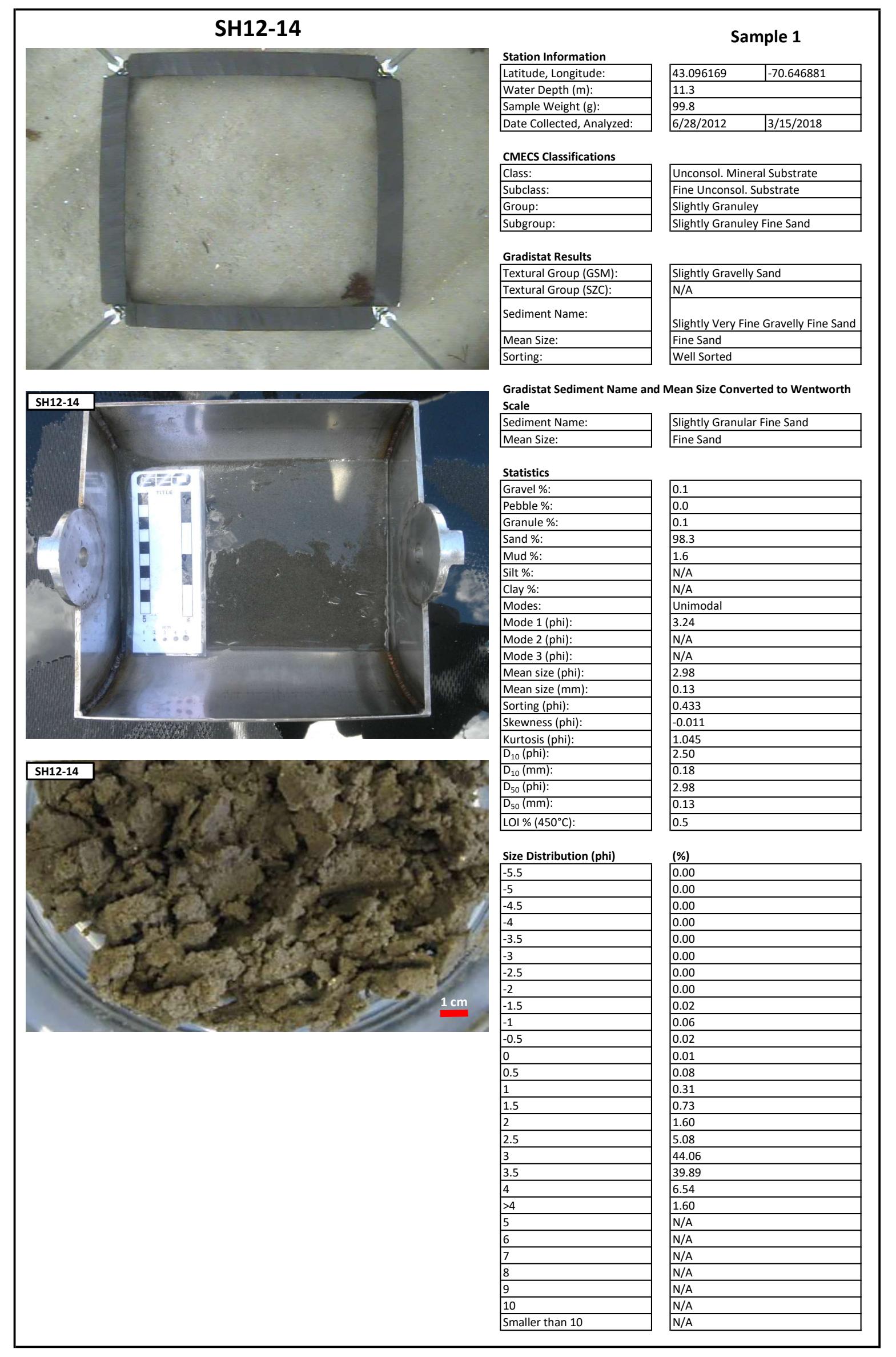




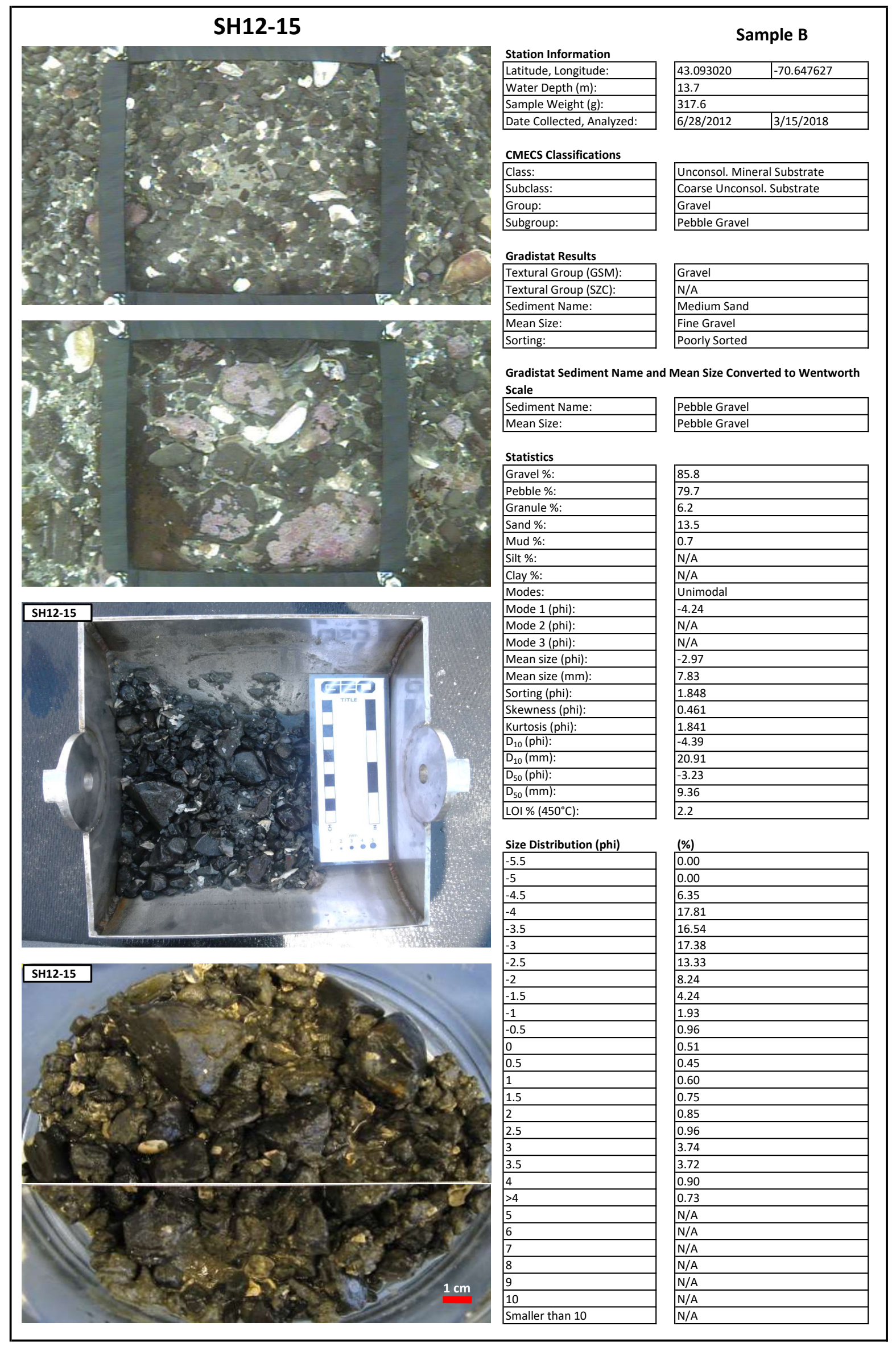




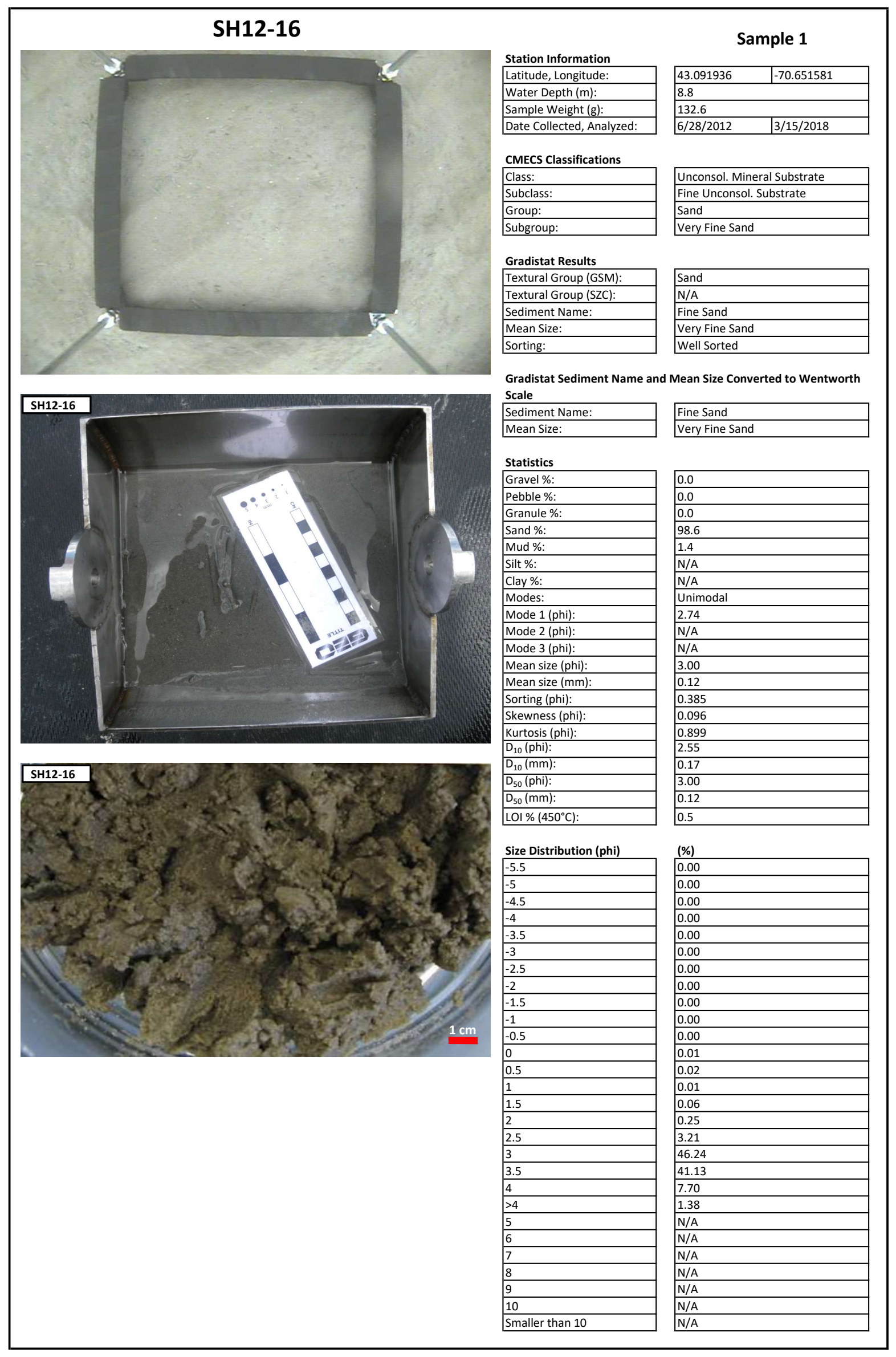




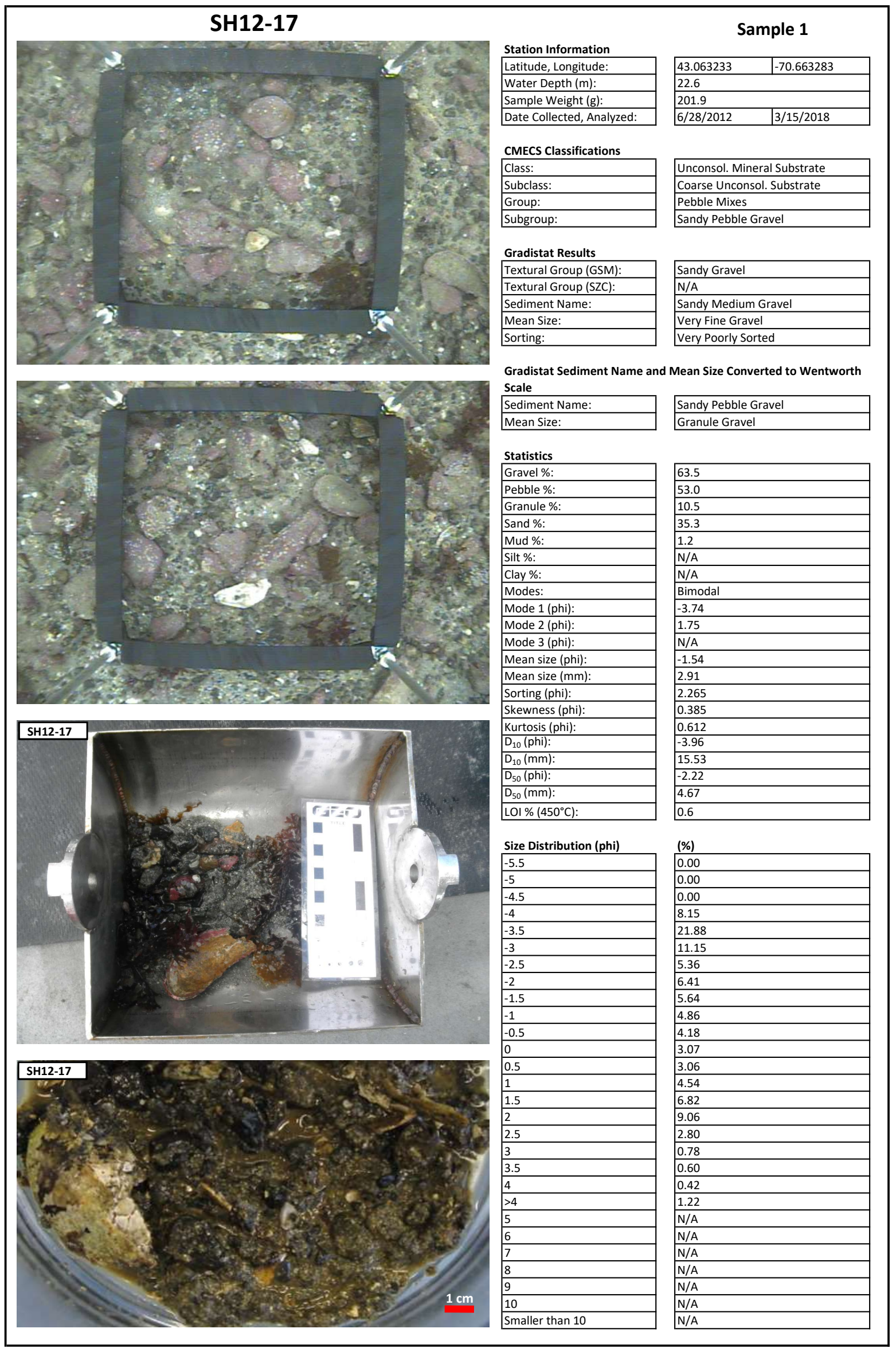




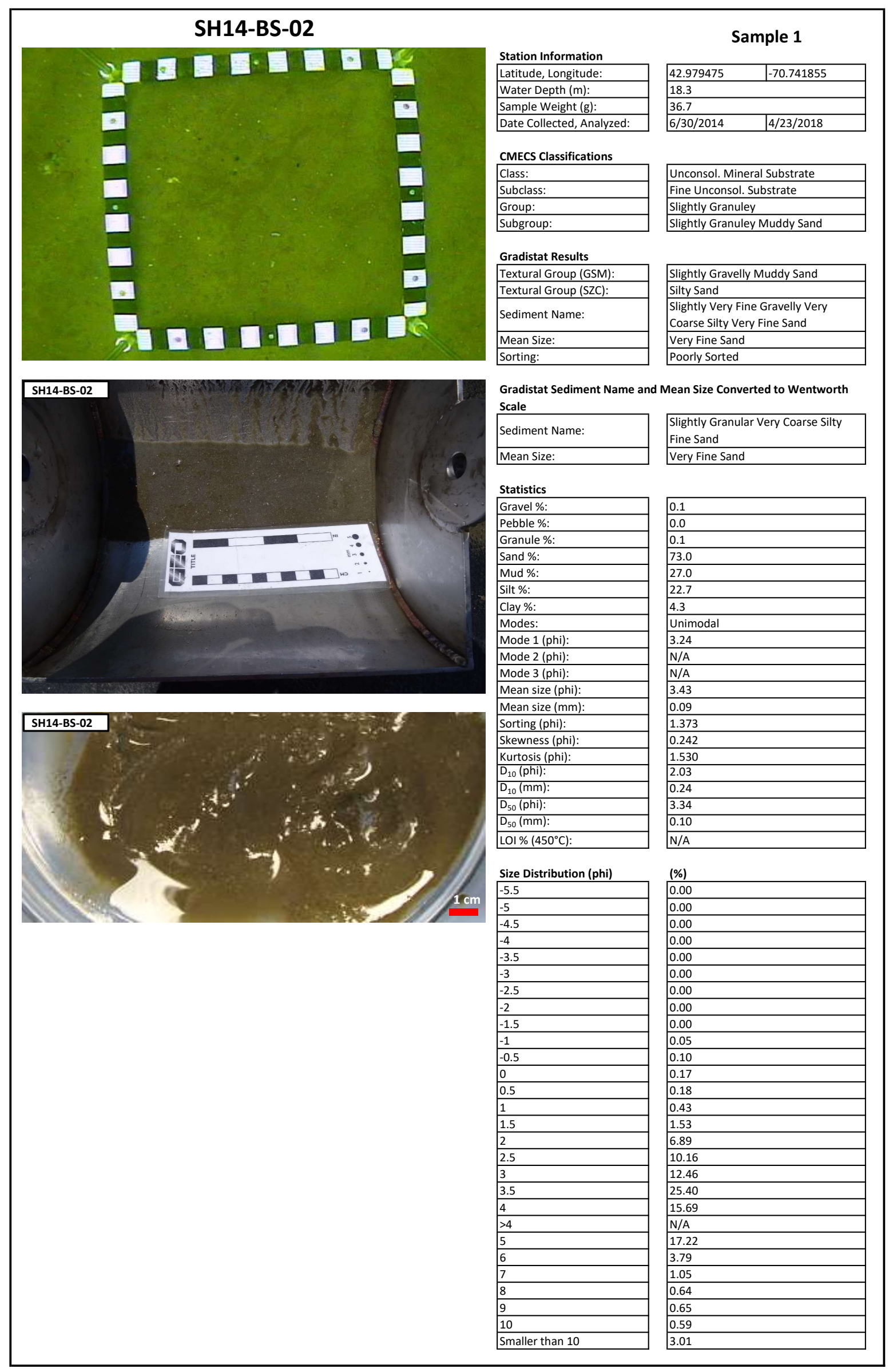




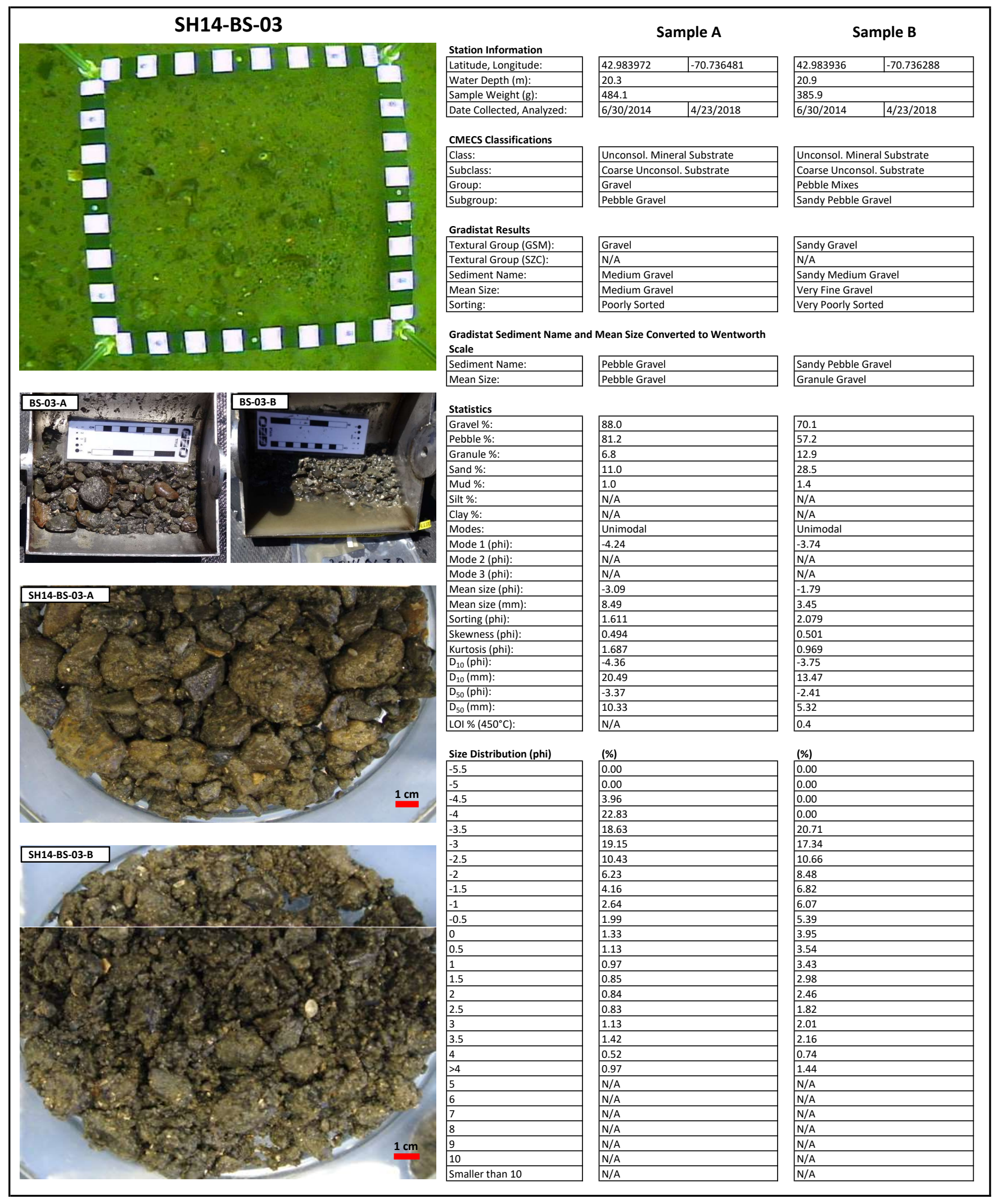




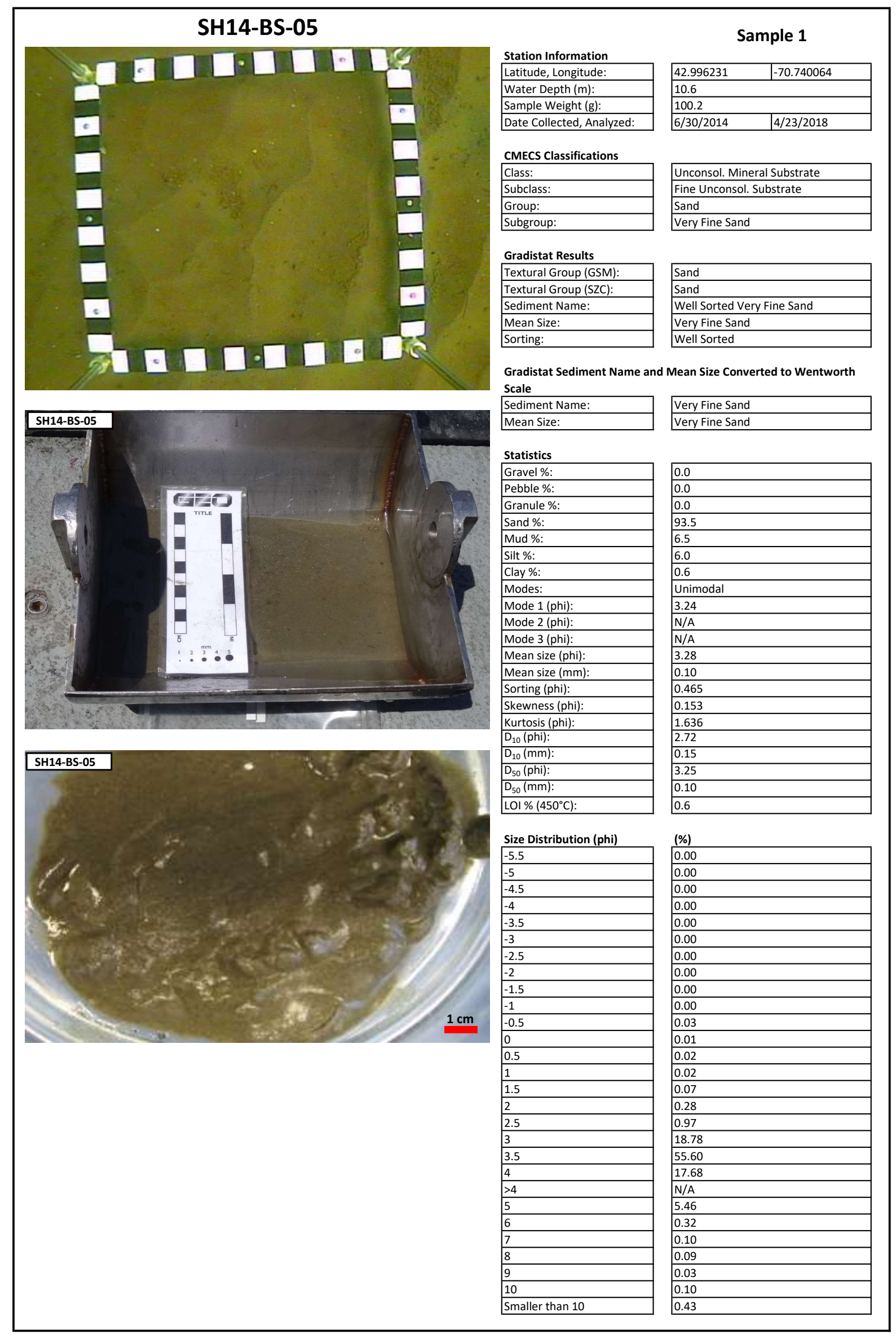




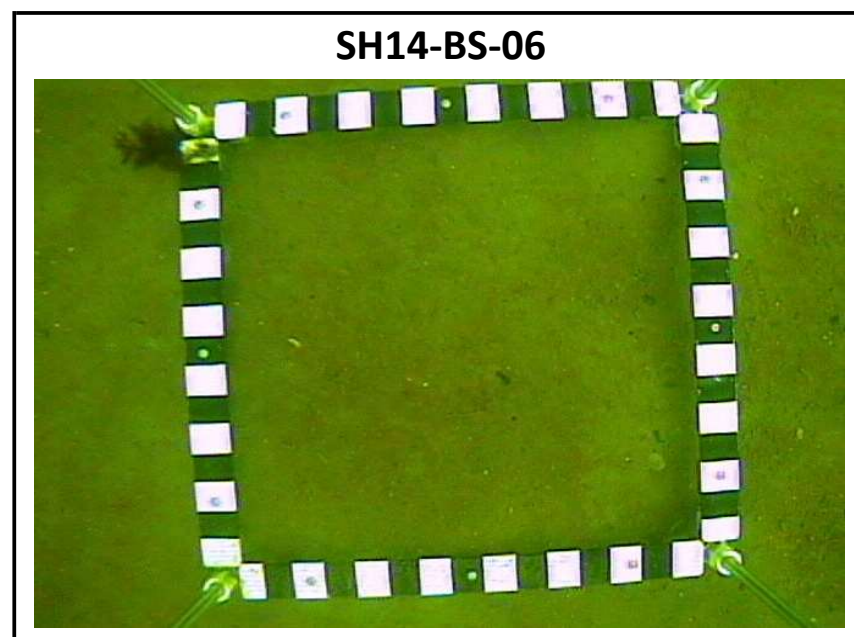

Station Information

Latitude, Longitude:

Water Depth $(\mathrm{m})$ :

Sample Weight (g):

Date Collected, Analyzed:

CMECS Classifications

Class:

Subclass:

Group:

Subgroup:

Sample 1

Gradistat Results

Textural Group (GSM):

Textural Group (SZC):

Sediment Name:

Mean Size:

Sorting:

\begin{tabular}{|l|l|}
\hline 42.995856 & -70.728489 \\
\hline 17.7 & \\
\hline 149.1 & $4 / 23 / 2018$ \\
\hline $7 / 1 / 2014$ & \\
\hline
\end{tabular}

Unconsol. Mineral Substrate

Fine Unconsol. Substrate

Slightly Pebbly

Slightly Pebbly Very Fine Sand

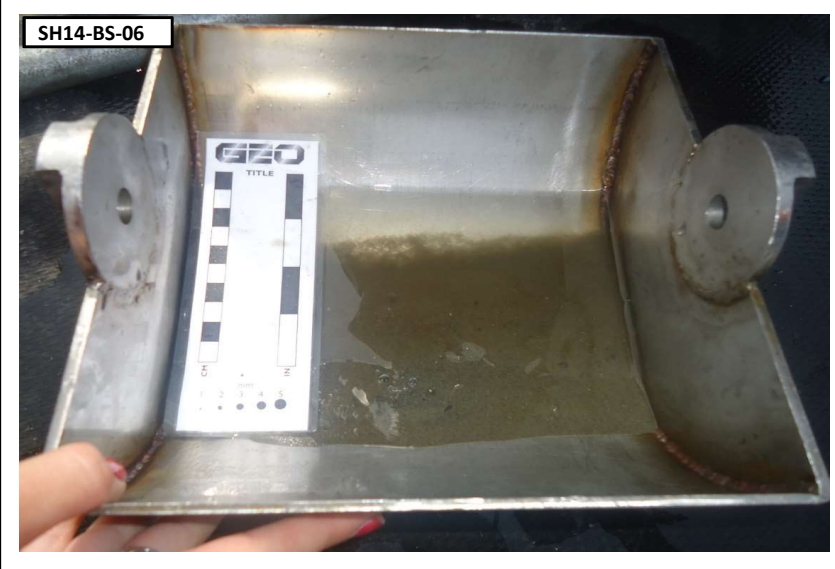

Gradistat Sediment Name and Mean Size Converted to Wentworth Scale

Sediment Name:

\begin{tabular}{|l}
\hline Sediment Nam Size: \\
\hline Mean Sil \\
\hline
\end{tabular}

Slightly Pebbly Very Fine Sand

\begin{tabular}{|l|}
\hline Slightly Gravelly Sand \\
\hline N/A \\
\hline Slightly Medium Gravelly Very Fine \\
Sand \\
\hline Very Fine Sand \\
\hline Moderately Well Sorted \\
\hline
\end{tabular}

Statistics

Gravel \%:

Pebble \%:

Granule \%:

Sand \%:

Mud \%:

Silt \%:

Clay \%:

Modes:

\begin{tabular}{l} 
Mode 1 (phi): \\
\hline
\end{tabular}

Mode 2 (phi):

Mode 3 (phi):

Mean size (phi):

Mean size $(\mathrm{mm})$ :

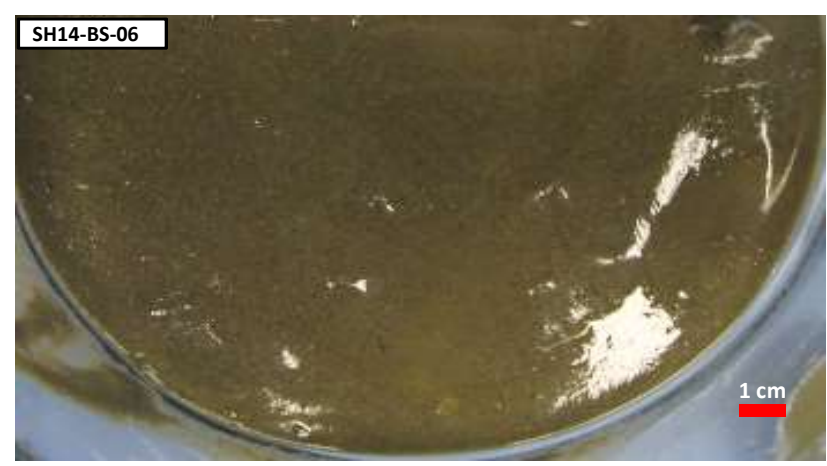

Sorting (phi):

Skewness (phi):

Kurtosis (phi):

$\mathrm{D}_{10}$ (phi):

$\mathrm{D}_{10}(\mathrm{~mm}):$

D

$\mathrm{D}_{50}(\mathrm{~mm}):$

LOI \% $\left(450^{\circ} \mathrm{C}\right)$

Very Fine Sand

Size Distribution (phi)

\begin{tabular}{l} 
Size Distribution (phi) \\
\begin{tabular}{|l|}
\hline-5.5 \\
\hline-5 \\
\hline-4.5 \\
\hline-4 \\
\hline-3.5 \\
\hline-3 \\
\hline-2.5 \\
\hline-2 \\
\hline-1.5 \\
\hline-1 \\
\hline-0.5 \\
\hline 0 \\
\hline 0.5 \\
\hline 1 \\
\hline 1.5 \\
\hline 2 \\
\hline 2.5 \\
\hline 3 \\
\hline 3.5 \\
\hline 4 \\
\hline$>4$ \\
\hline 5 \\
\hline 6 \\
\hline 7 \\
\hline 8 \\
\hline 9 \\
\hline 10 \\
\hline Smaller than 10 \\
\hline
\end{tabular} \\
\hline
\end{tabular}

\begin{tabular}{|l|}
\hline 2.6 \\
\hline 1.9 \\
\hline 0.7 \\
\hline 92.6 \\
\hline 4.8 \\
\hline 3.1 \\
\hline 1.7 \\
\hline Unimodal \\
\hline 3.24 \\
\hline N/A \\
\hline N/A \\
\hline 3.03 \\
\hline 0.12 \\
\hline 0.672 \\
\hline-0.308 \\
\hline 1.896 \\
\hline 2.26 \\
\hline 0.21 \\
\hline 3.09 \\
\hline 0.12 \\
\hline 0.7 \\
\hline
\end{tabular}

(\%)

\begin{tabular}{l}
$(\%)$ \\
\begin{tabular}{|l|}
\hline 0.00 \\
\hline 0.00 \\
\hline 0.00 \\
\hline 0.00 \\
\hline 0.00 \\
\hline 1.24 \\
\hline 0.00 \\
\hline 0.67 \\
\hline 0.18 \\
\hline 0.53 \\
\hline 0.43 \\
\hline 0.46 \\
\hline 0.66 \\
\hline 0.80 \\
\hline 0.96 \\
\hline 1.72 \\
\hline 4.24 \\
\hline 29.44 \\
\hline 45.88 \\
\hline 8.01 \\
\hline N/A \\
\hline 2.05 \\
\hline 0.51 \\
\hline 0.29 \\
\hline 0.29 \\
\hline 0.22 \\
\hline 0.24 \\
\hline 1.19 \\
\hline \\
\hline
\end{tabular} \\
\hline
\end{tabular}




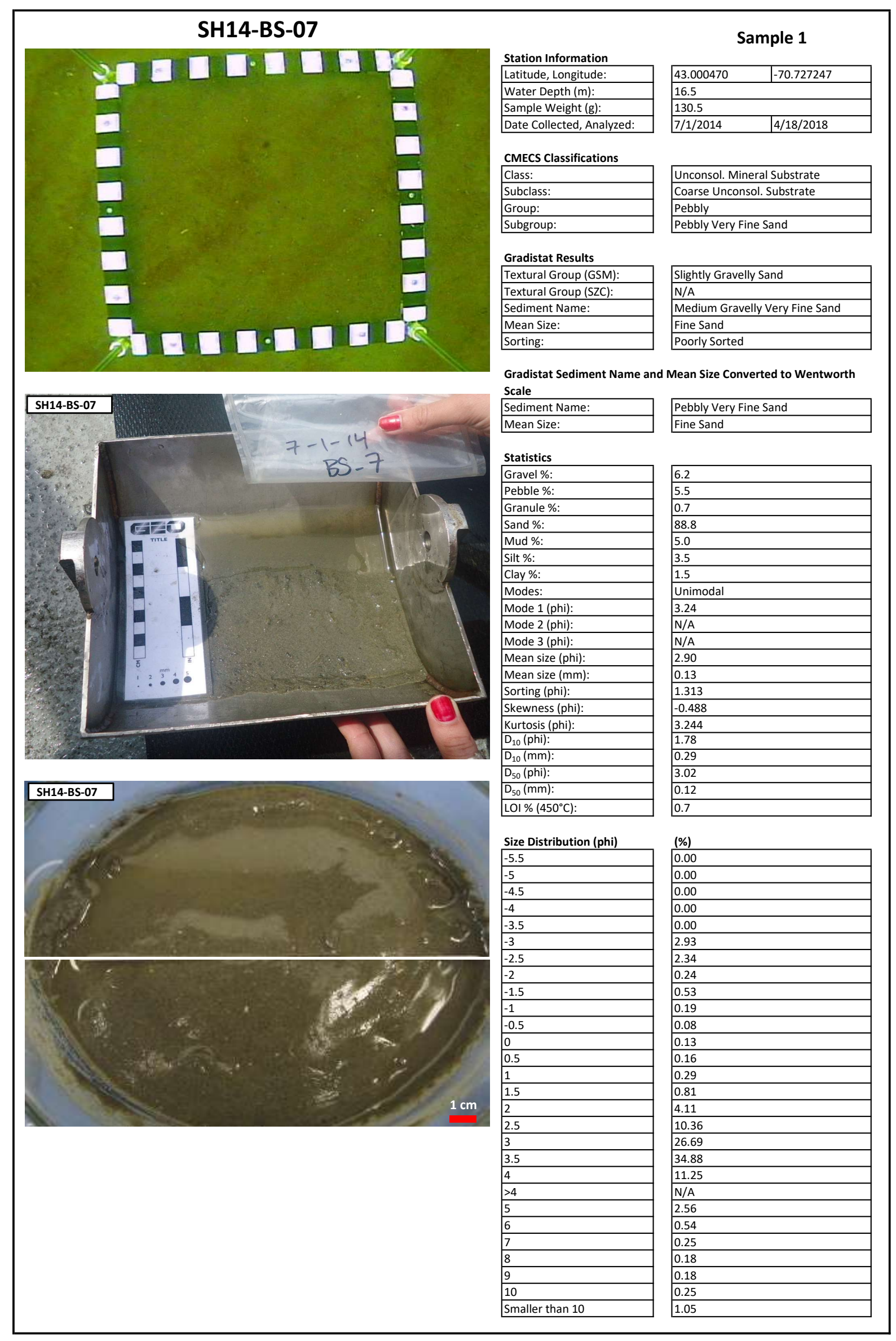




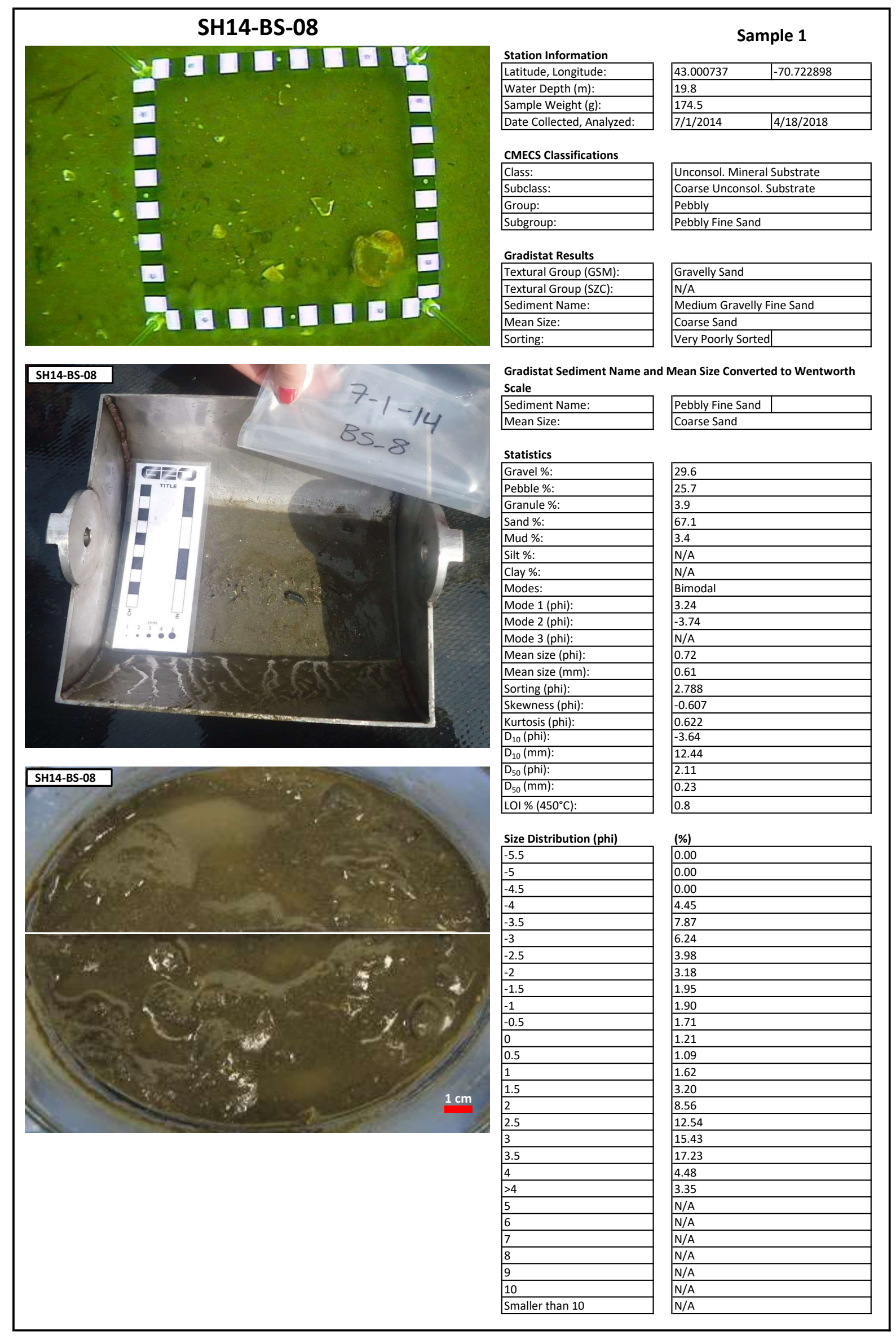




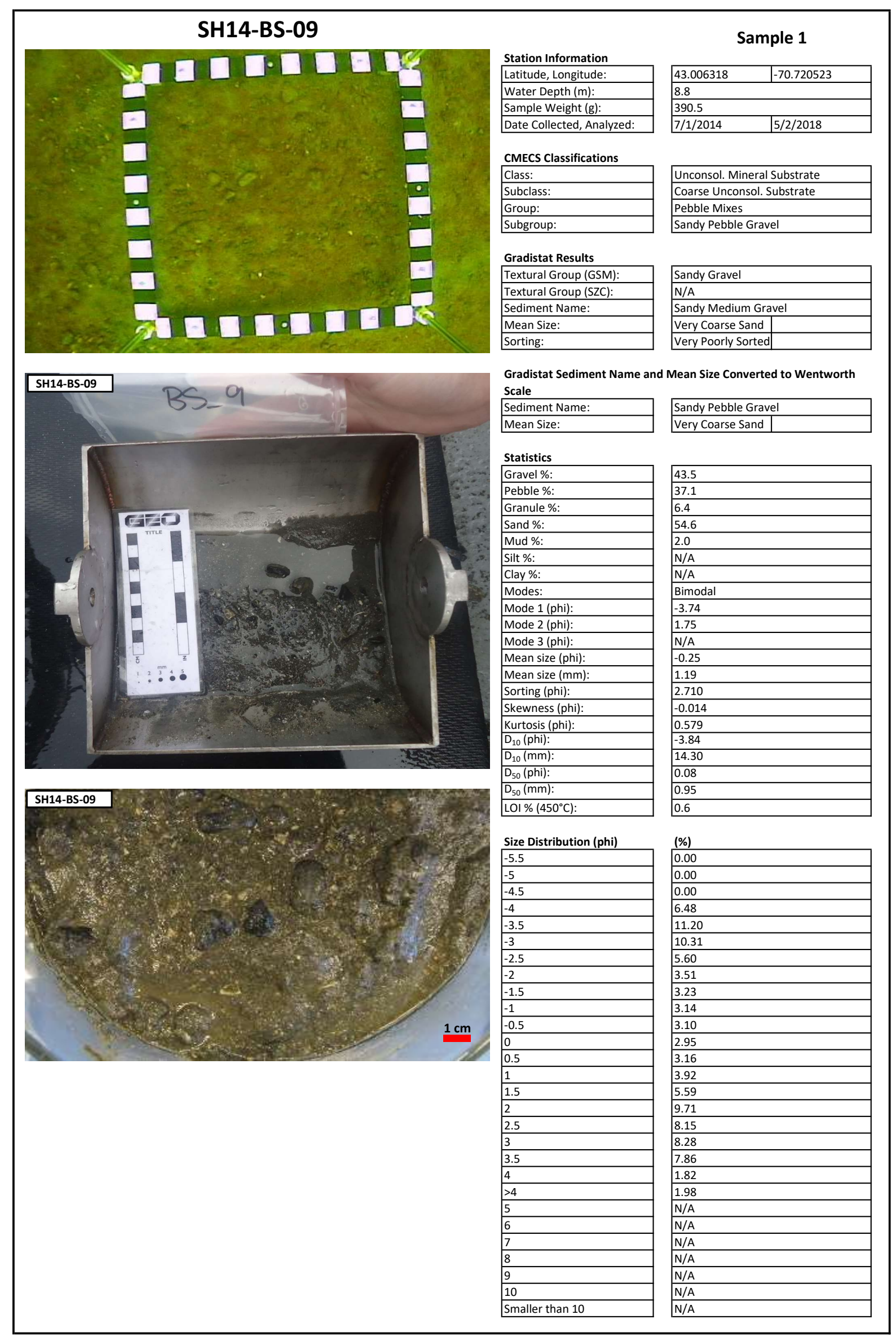




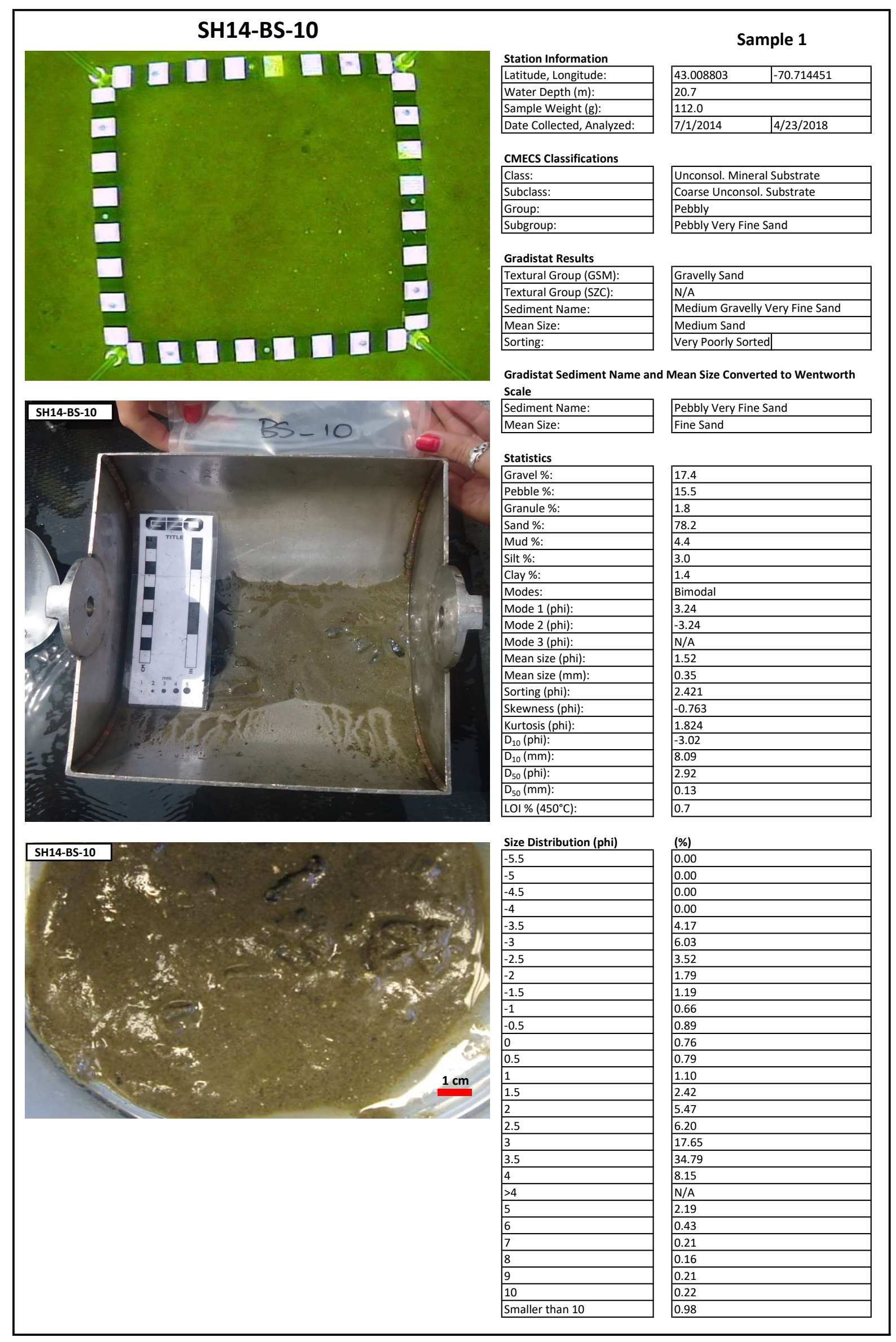



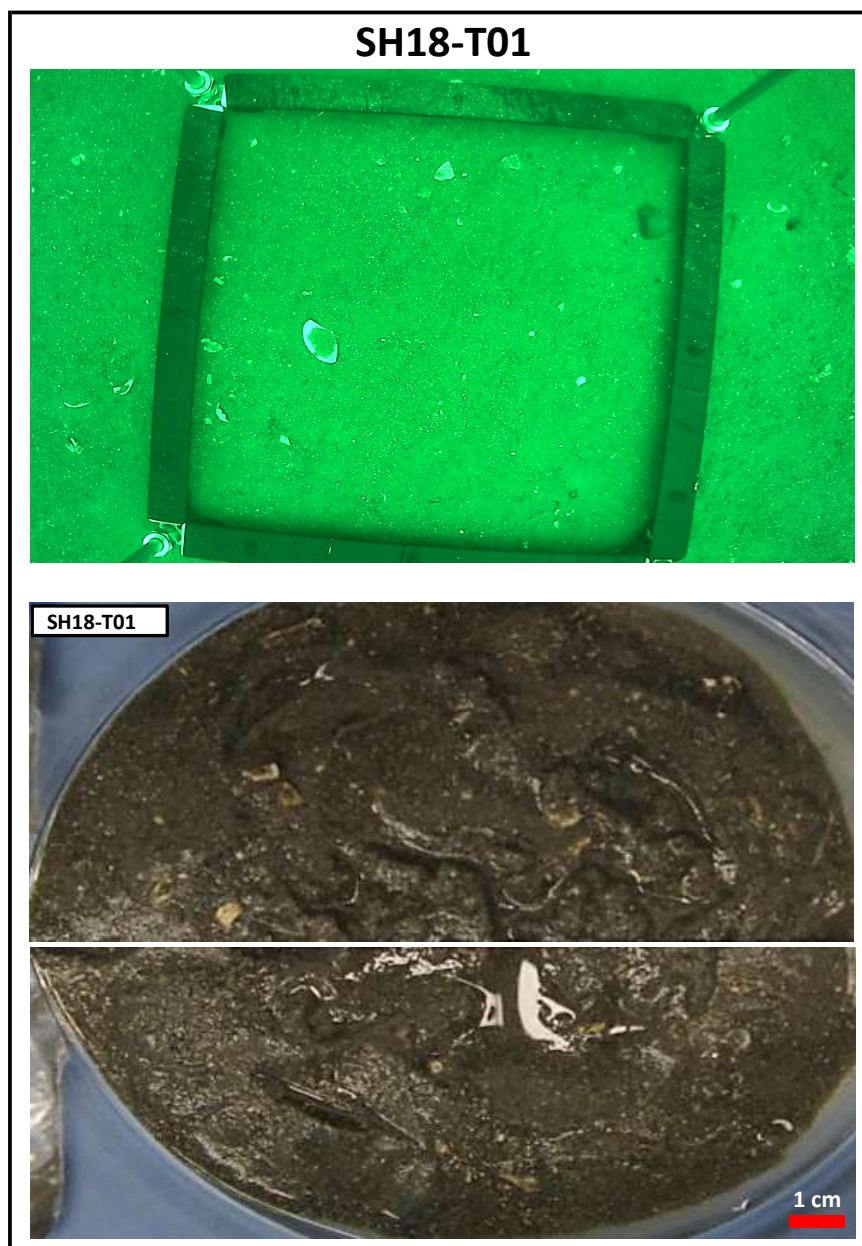

Station Information

Latitude, Longitude

Water Depth $(\mathrm{m})$ :

Sample Weight (g):

Date Collected, Analyzed:

CMECS Classifications

Class:

Subclass:

Group:

Subgroup:

Sample 1

Gradistat Results

Textural Group (GSM):

Textural Group (SZC):

Sediment Name:

Mean Size:

Sorting:

\begin{tabular}{|l|l|}
\hline 43.110750 & -70.620768 \\
\hline 25.0 & \\
\hline 141.0 & $7 / 16 / 2018$ \\
\hline $7 / 10 / 2018$ & \\
\hline
\end{tabular}

Unconsol. Mineral Substrate

Fine Unconsol. Substrate

Slightly Pebbly

Slightly Pebbly Fine Sand

Gradistat Sediment Name and Mean Size Converted to Wentworth

Scale

Sediment Name:

Mean Size:

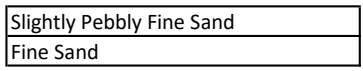

Statistics

Gravel \%:

Pebble \%:

Granule \%:

Sand \%:

Mud \%:

Silt \%:

Clay \%:

Modes:

Mode 1 (phi):

Mode 2 (phi):

Mode 3 (phi):

Mean size (phi):

Mean size $(\mathrm{mm})$ :

Sorting (phi):

Skewness (phi):

Kurtosis (phi):

\begin{tabular}{|l|}
\hline Kurtosis (phi): \\
\hline$D_{10}(p h i):$ \\
\hline$D_{10}(\mathrm{~mm}):$ \\
\hline$D_{50}(p h i):$ \\
\hline$D_{50}(\mathrm{~mm}):$ \\
\hline LOI \% $\left(450^{\circ} \mathrm{C}\right):$ \\
\hline
\end{tabular}

\begin{tabular}{|l|}
\hline Slightly Gravelly Sand \\
\hline N/A \\
\hline Slightly Fine Gravelly Fine Sand \\
\hline Fine Sand \\
\hline Moderately Well Sorted \\
\hline
\end{tabular}

Moderately Well Sorted

Size Distribution (phi)

\begin{tabular}{|l|}
\hline-5.5 \\
\hline-5 \\
\hline-4.5 \\
\hline-4 \\
\hline-3.5 \\
\hline-3 \\
\hline-2.5 \\
\hline-2 \\
\hline-1.5 \\
\hline-1 \\
\hline-0.5 \\
\hline 0 \\
\hline 0.5 \\
\hline 1 \\
\hline 1.5 \\
\hline 2 \\
\hline 2.5 \\
\hline 3 \\
\hline 3.5 \\
\hline 4 \\
\hline$>4$ \\
\hline 5 \\
\hline 6 \\
\hline 7 \\
\hline 8 \\
\hline 9 \\
\hline 10 \\
\hline Smaller than 10 \\
\hline
\end{tabular}

\begin{tabular}{|l|}
\hline 1.4 \\
\hline 1.4 \\
\hline 0.0 \\
\hline 96.2 \\
\hline 2.5 \\
\hline $\mathrm{N} / \mathrm{A}$ \\
\hline $\mathrm{N} / \mathrm{A}$ \\
\hline Unimodal \\
\hline 2.74 \\
\hline $\mathrm{N} / \mathrm{A}$ \\
\hline $\mathrm{N} / \mathrm{A}$ \\
\hline 2.73 \\
\hline 0.15 \\
\hline 0.658 \\
\hline-0.032 \\
\hline 1.042 \\
\hline 1.88 \\
\hline 0.27 \\
\hline 2.74 \\
\hline 0.15 \\
\hline 0.8 \\
\hline
\end{tabular}

\begin{tabular}{l} 
(\%) \\
\hline 0.00 \\
\hline 0.00 \\
\hline 0.00 \\
\hline 0.00 \\
\hline 0.00 \\
\hline 0.00 \\
\hline 1.03 \\
\hline 0.34 \\
\hline 0.00 \\
\hline 0.02 \\
\hline 0.11 \\
\hline 0.26 \\
\hline 0.31 \\
\hline 0.44 \\
\hline 1.35 \\
\hline 8.02 \\
\hline 21.51 \\
\hline 32.94 \\
\hline 23.42 \\
\hline 7.80 \\
\hline 2.45 \\
\hline N/A \\
\hline N/A \\
\hline N/A \\
\hline N/A \\
\hline N/A \\
\hline N/A \\
\hline N/A \\
\hline \\
\hline
\end{tabular}




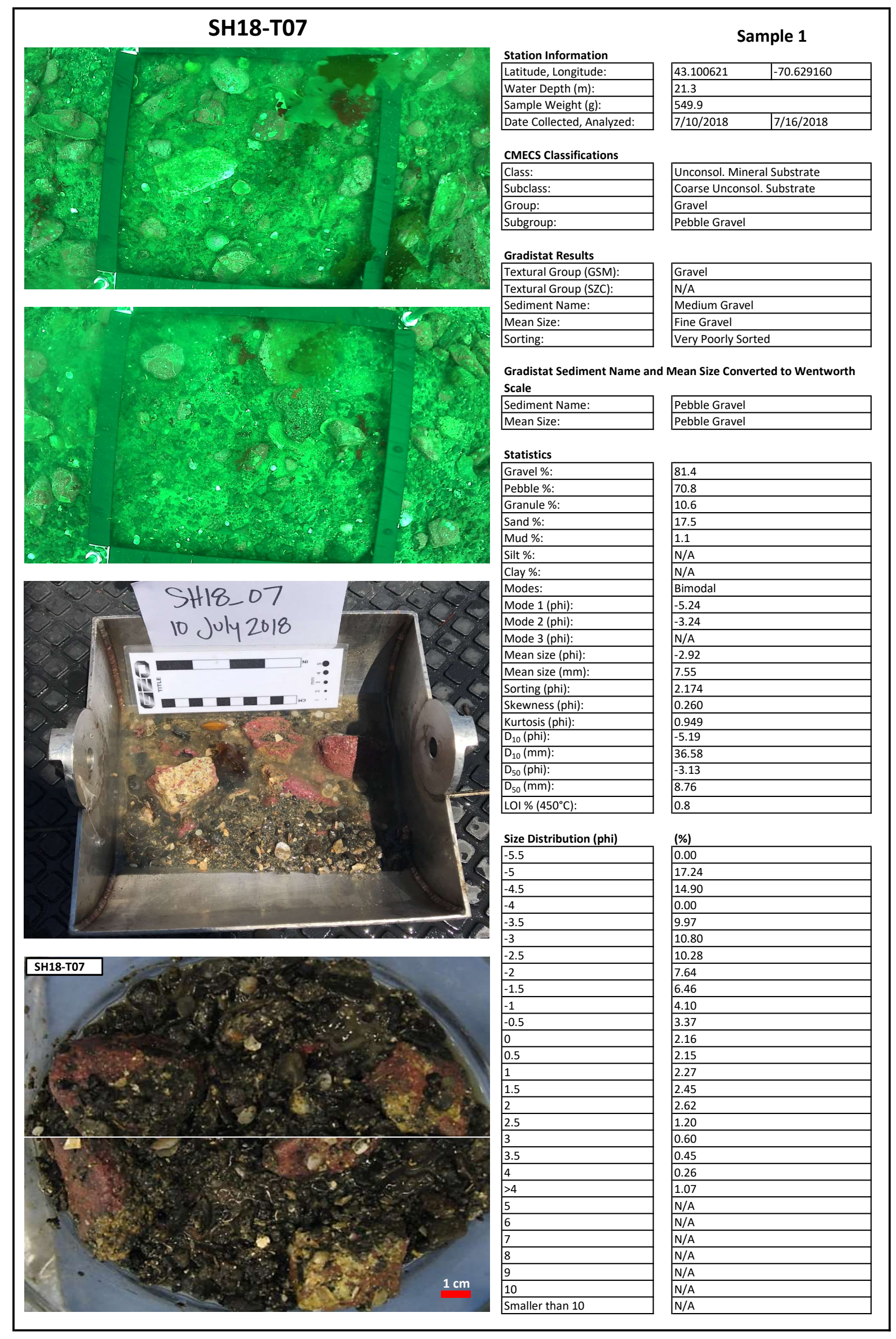




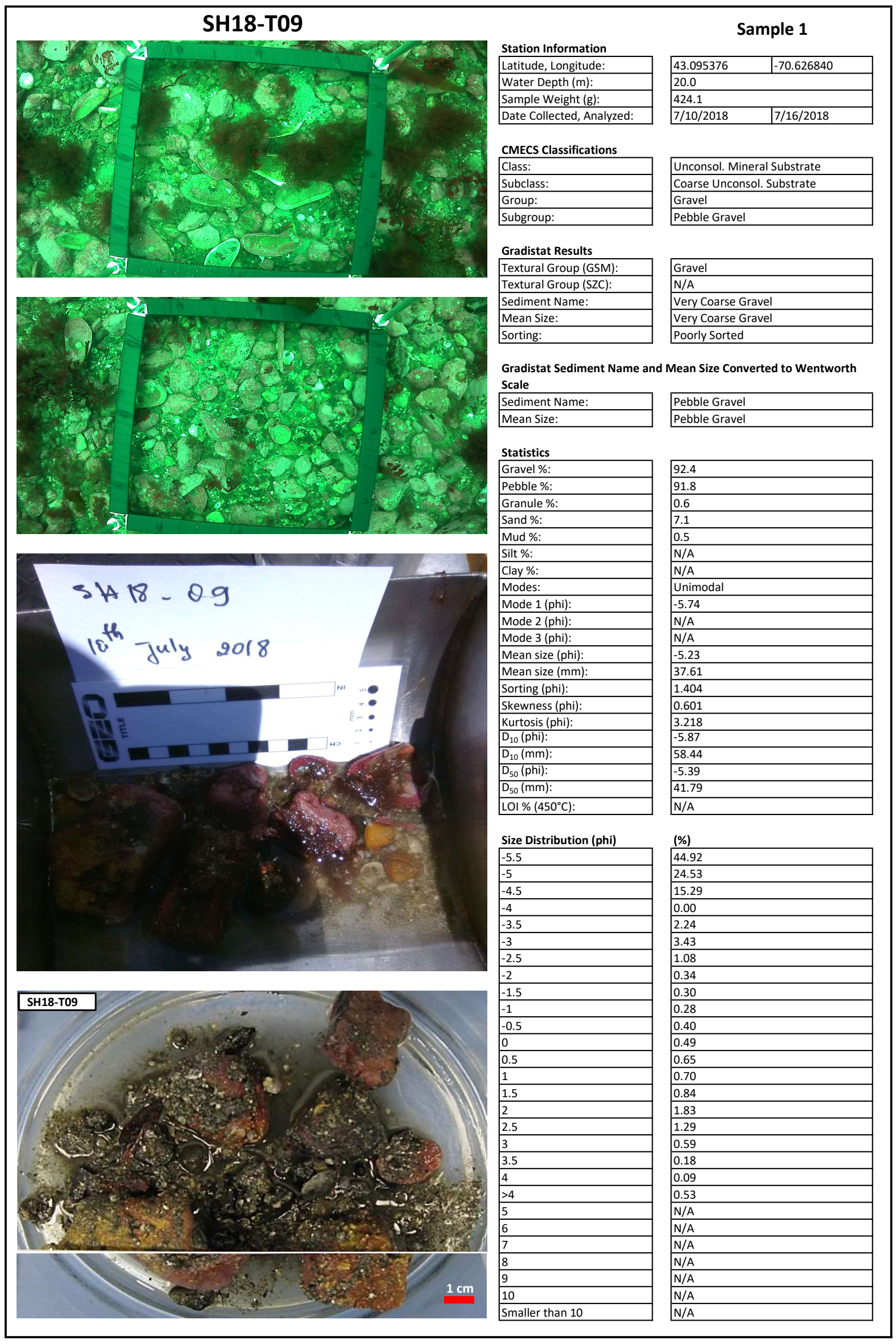




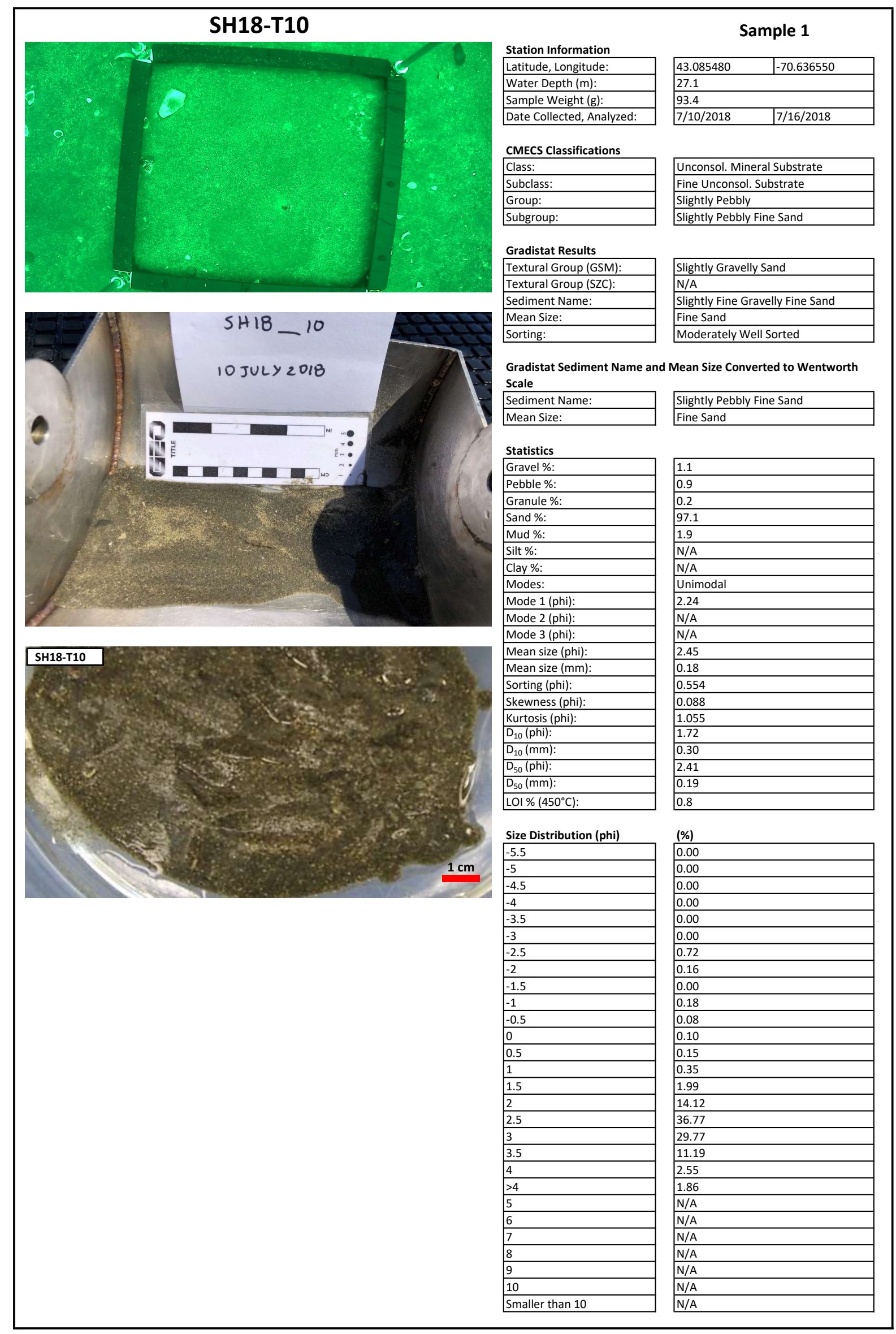




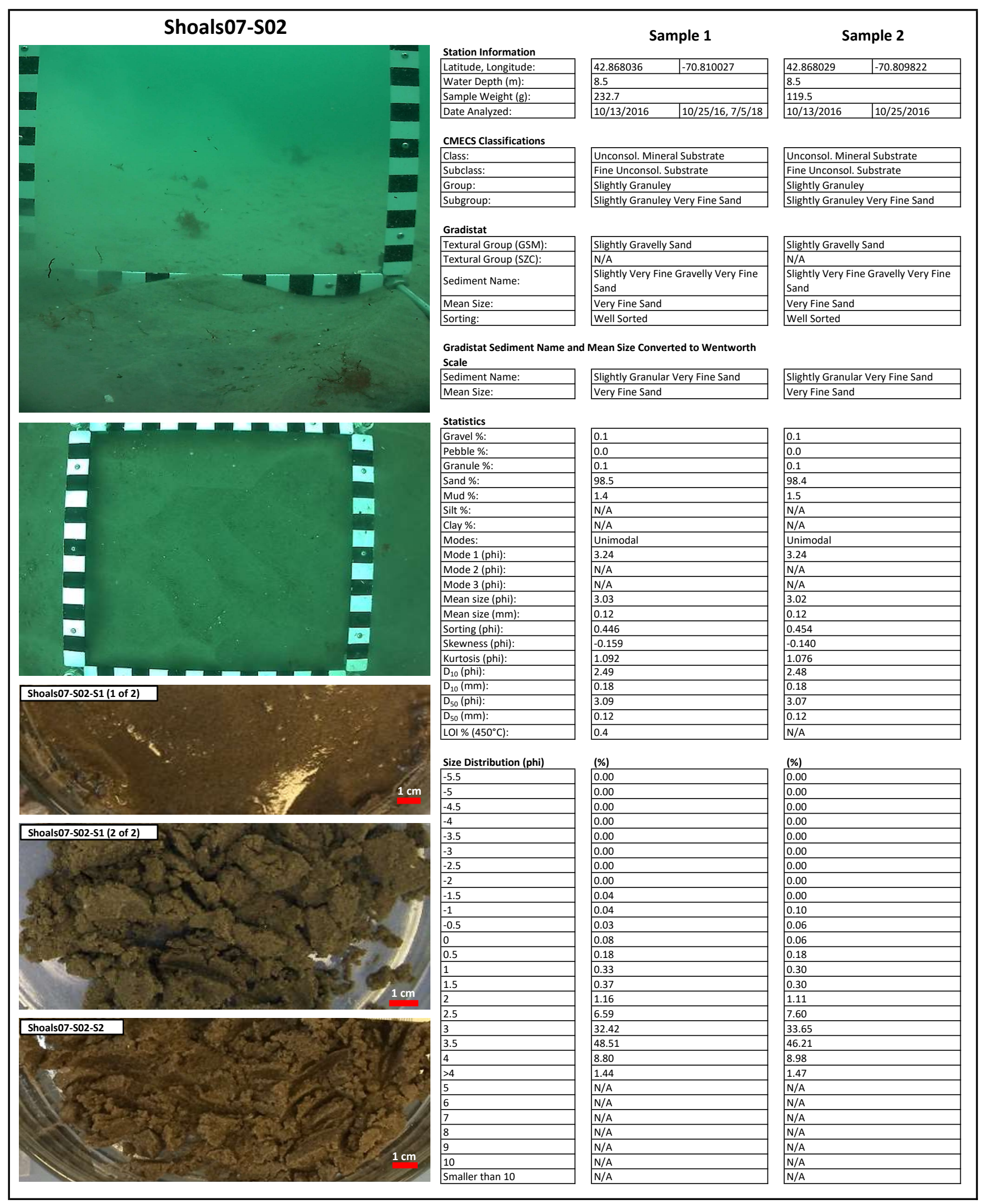




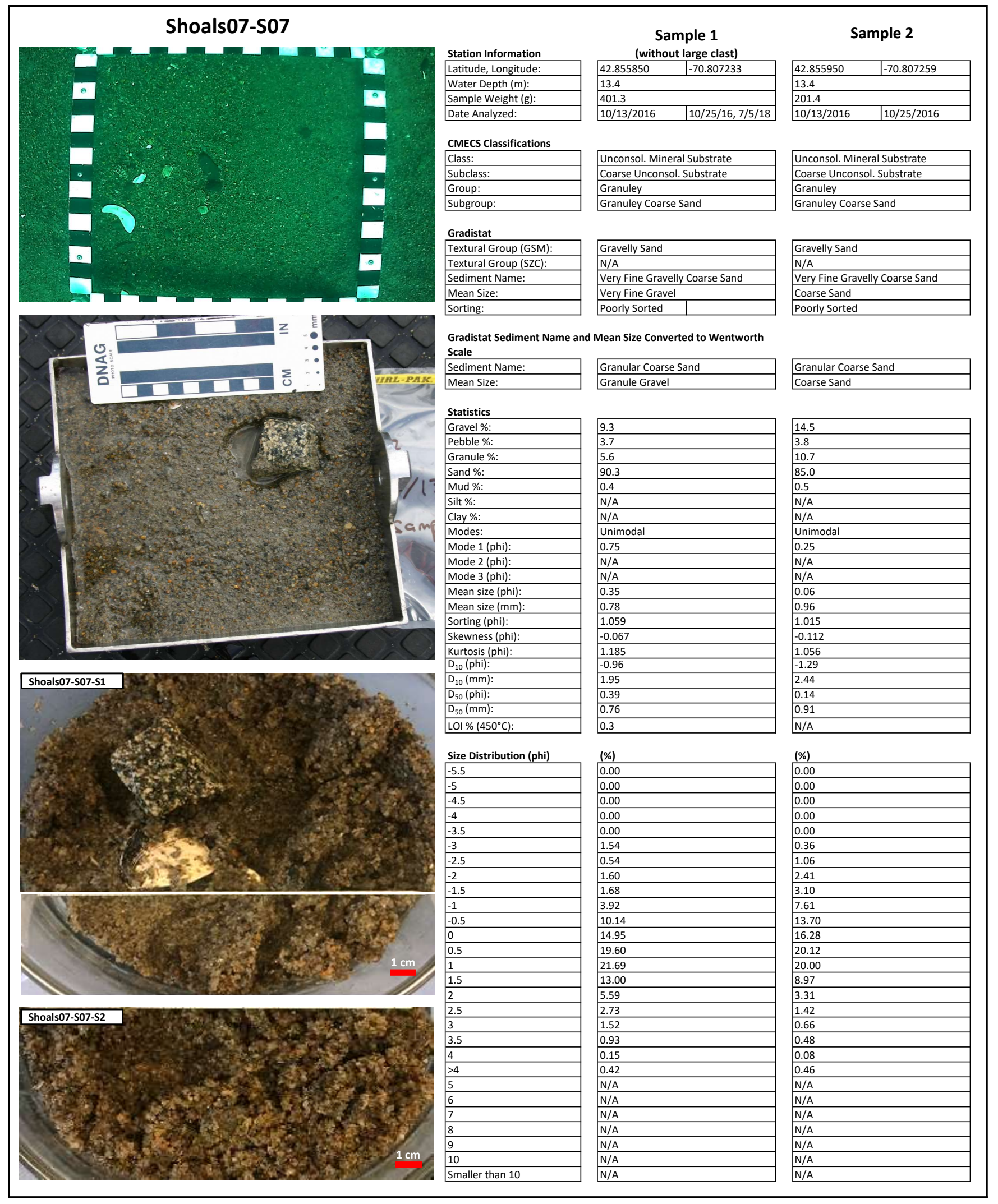




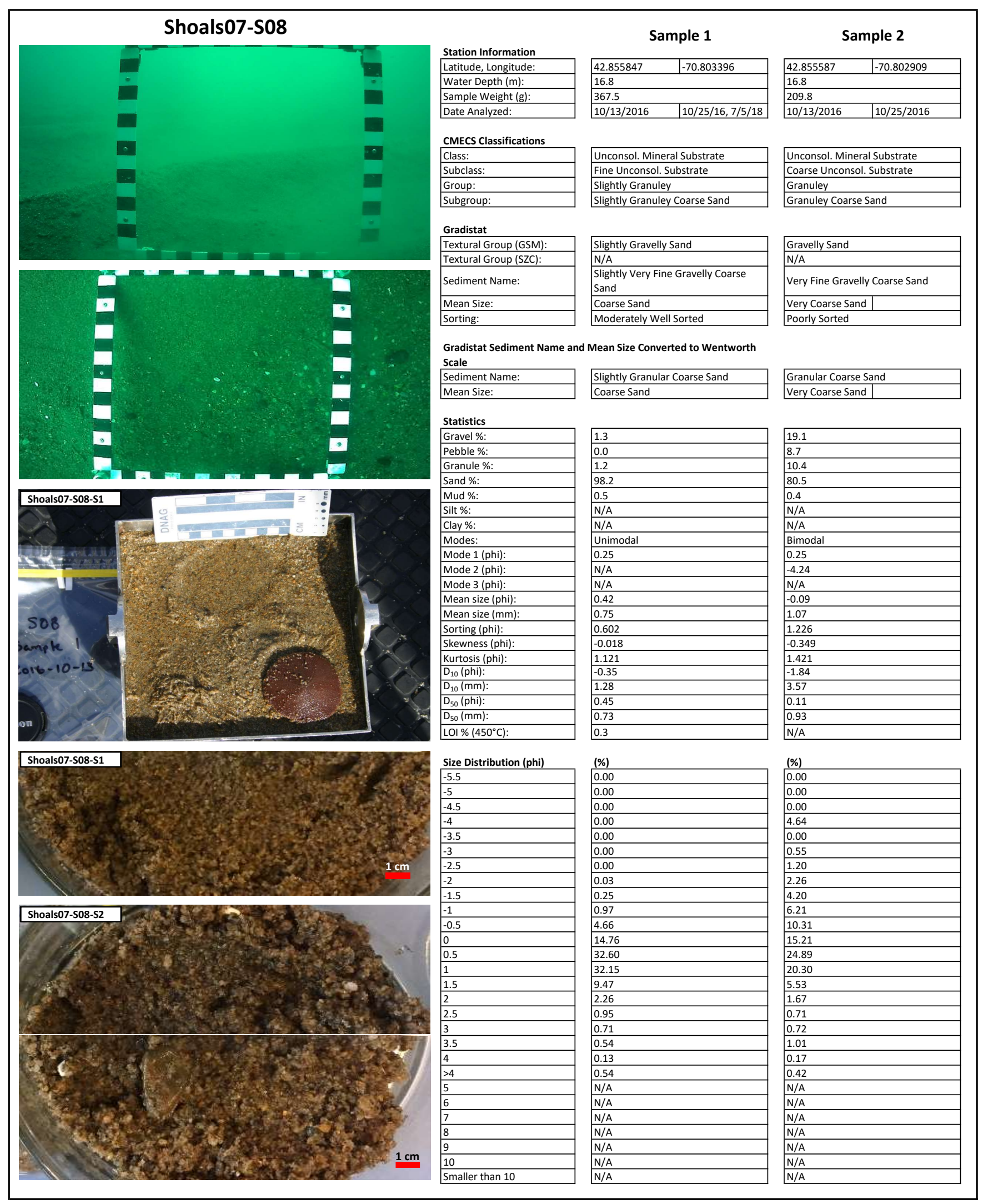




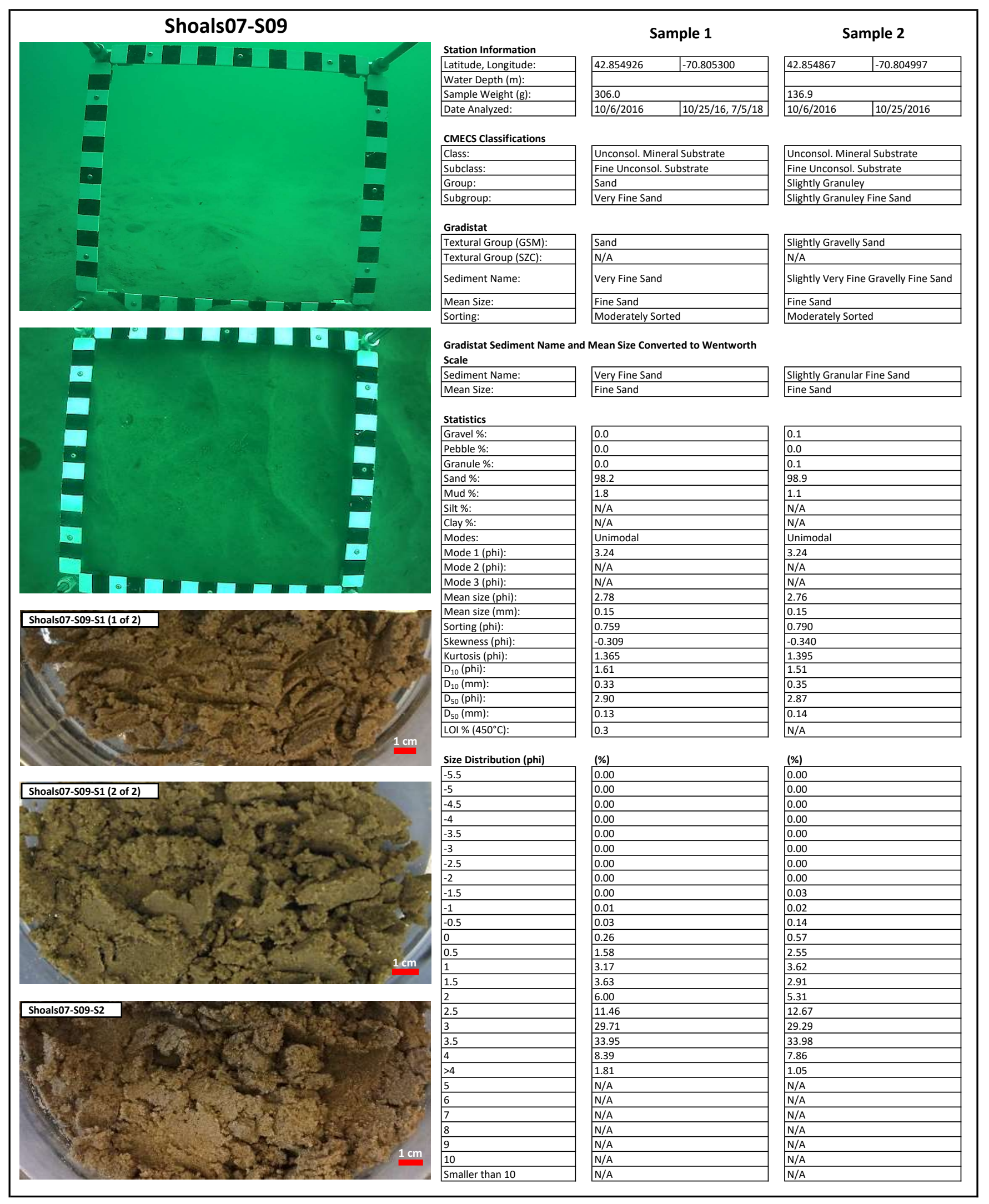




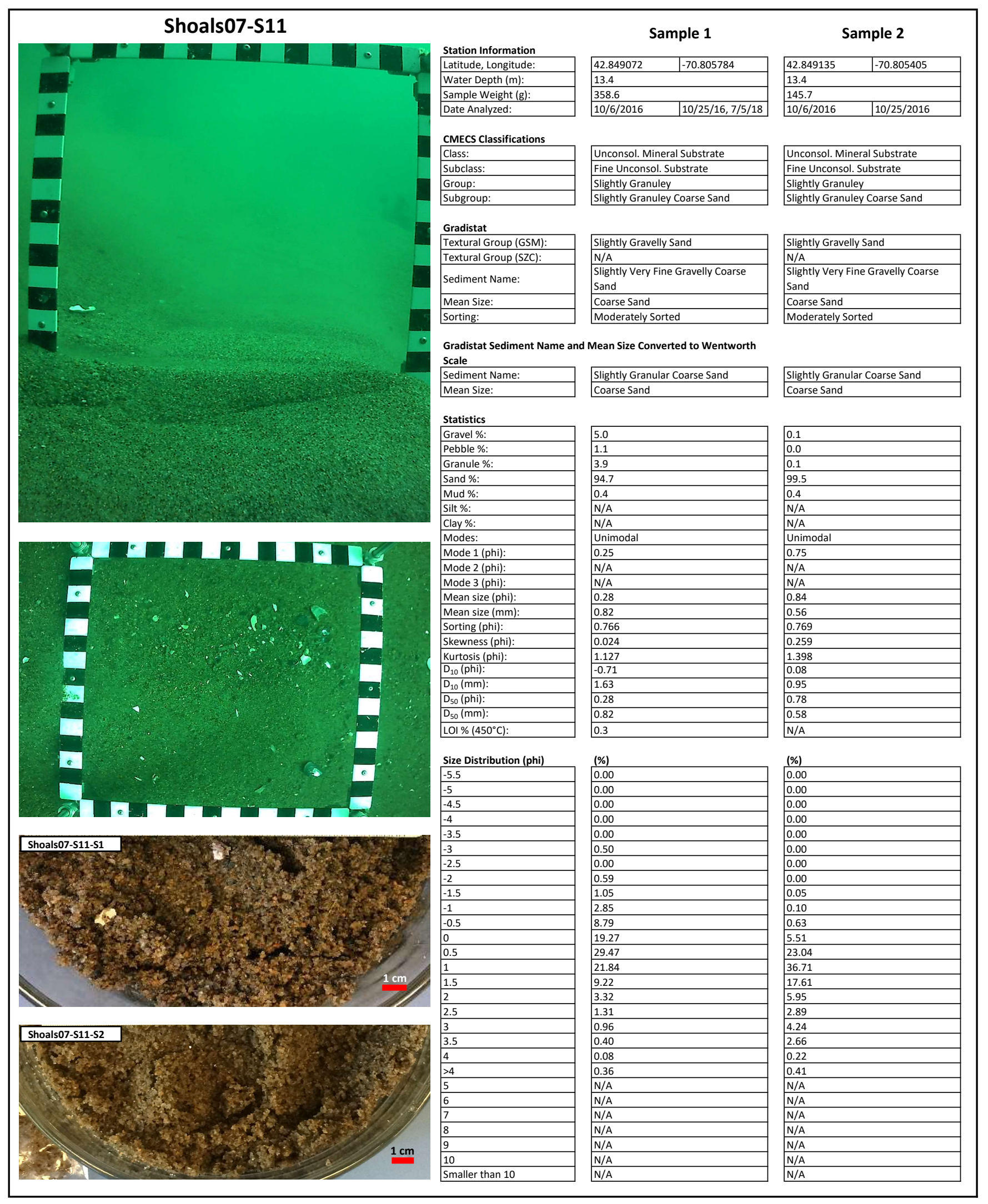




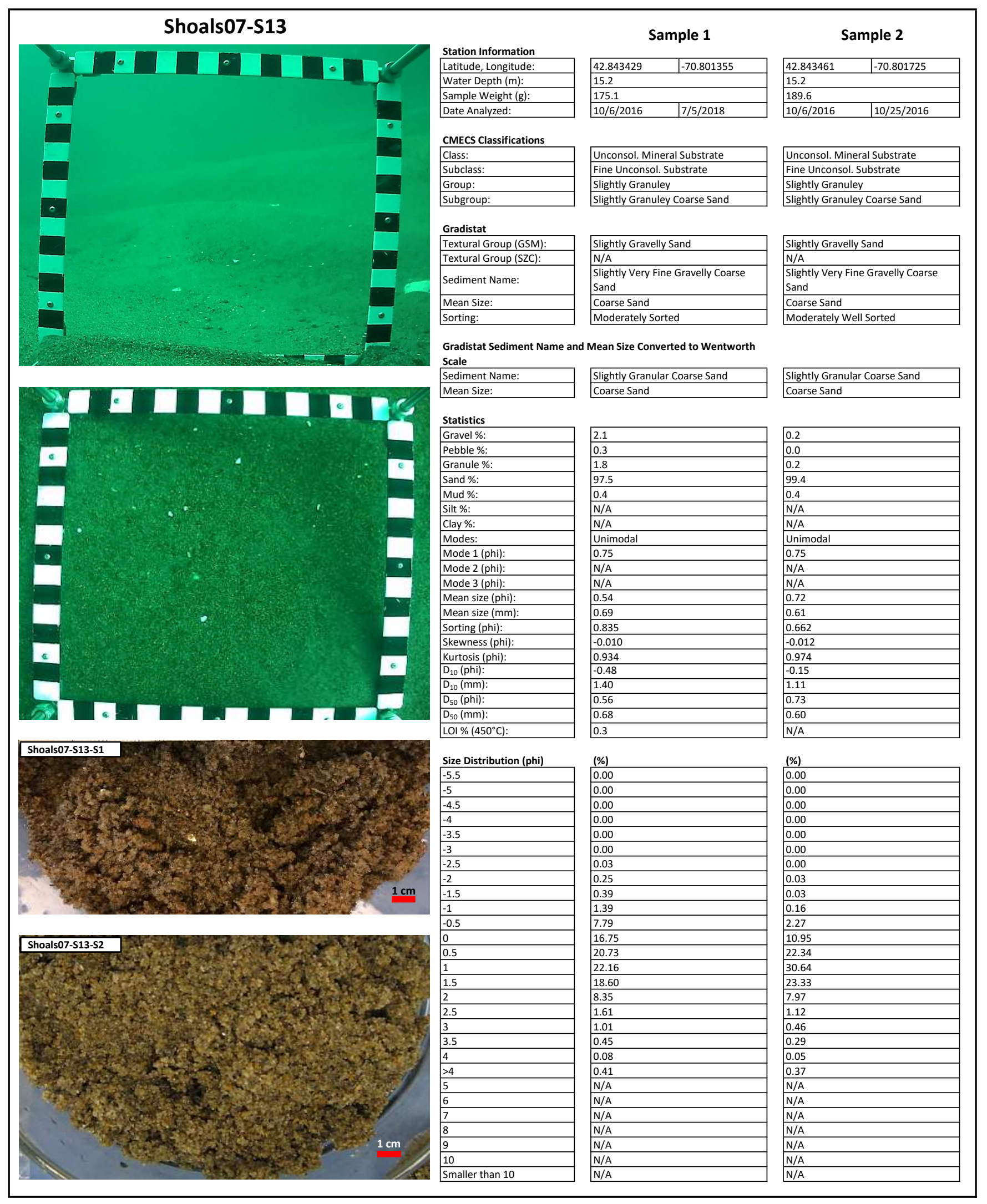




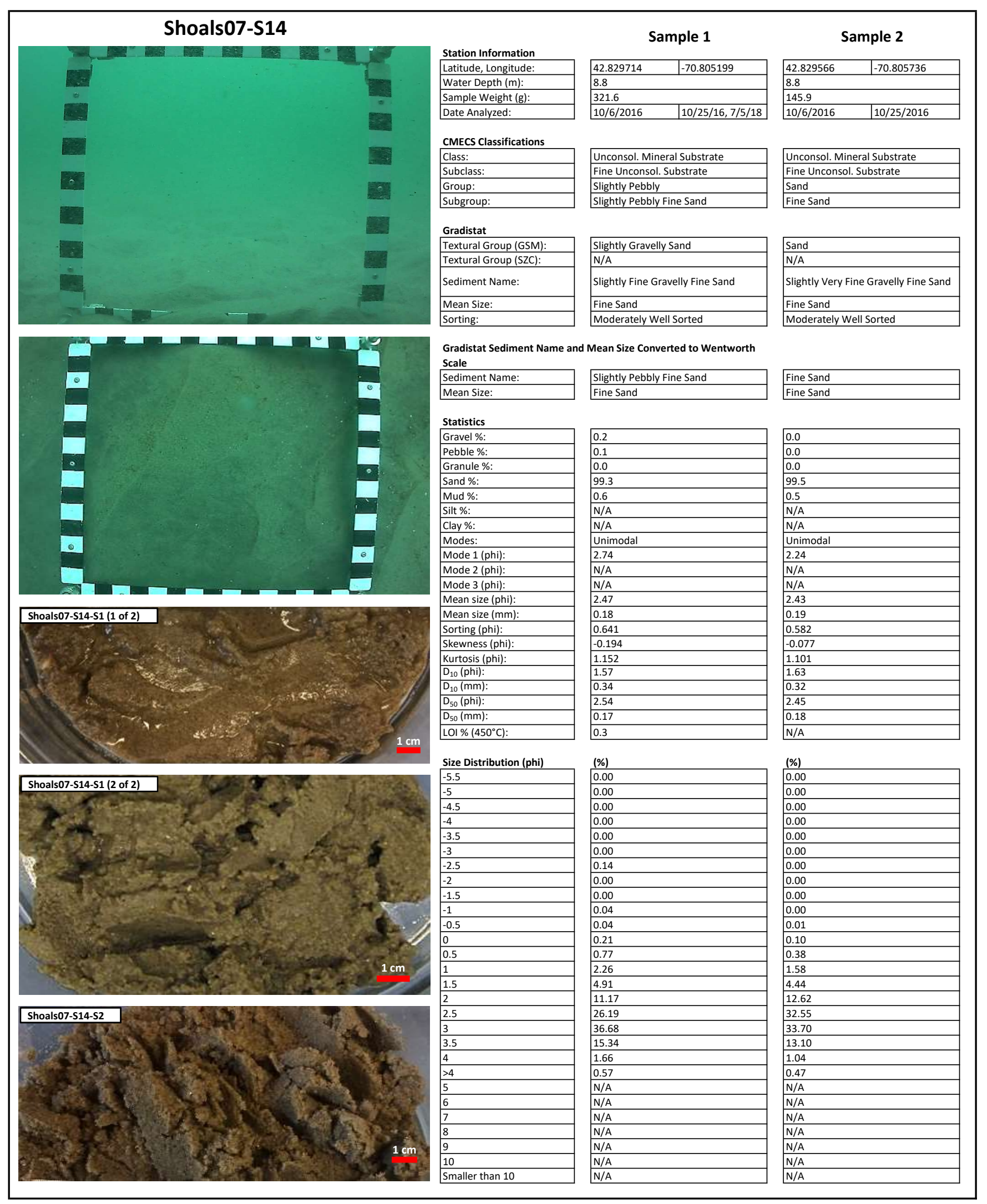

